\title{
Total Dissolved Gas, Barometric Pressure, and Water Temperature Data, Lower Columbia River, Oregon and Washington, 1996
}

U.S. GEOLOGICAL SURVEY Open-File Report 96-662A

Prepared in cooperation with the U.S. Army Corps of Engineers
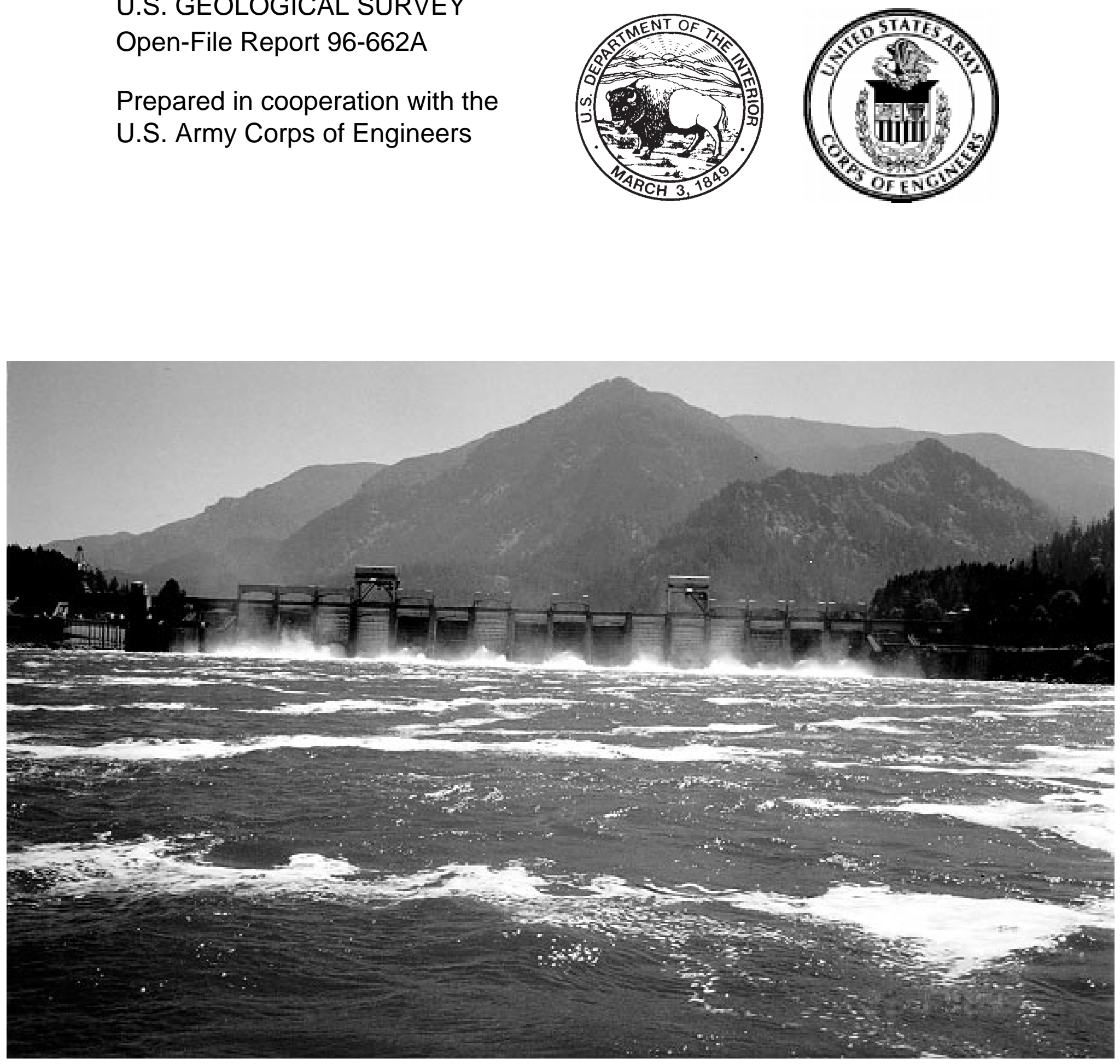
Cover photograph. Columbia River downstream of Bonneville Dam, Summer 1995. (Photograph by Tom Edwards, U. S. Geological Survey.) 


\section{Total Dissolved Gas, Barometric Pressure, and Water Temperature Data, Lower Columbia River, Oregon and Washington, 1996}

By Dwight Q. Tanner, Howard E. Harrison, and Stuart W. McKenzie

U.S. GEOLOGICAL SURVEY OPEN-FILE Report 96-662A

Prepared in cooperation with the U.S. Army Corps of Engineers 


\title{
U.S. DEPARTMENT OF THE INTERIOR BRUCE BABBITT, Secretary
}

\author{
U.S. GEOLOGICAL SURVEY \\ GORDON P. EATON, Director
}

Any use of trade, product, or firm names in this publication is for descriptive purposes only and does not imply endorsement by the U.S. Government

For additional information write to:

District Chief

U.S. Geological Survey, WRD 10615 S.E. Cherry Blossom Drive Portland, Oregon 97216
Copies of this report can

be purchased from:

U.S. Geological Survey

Earth Science Information Center Open-File Reports Section

Box 25286, MS 517

Denver Federal Center

Denver, Colorado 80225 


\section{CONTENTS}

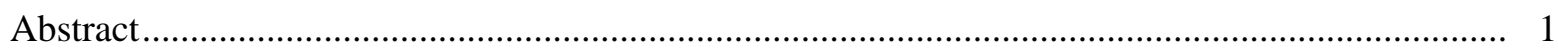

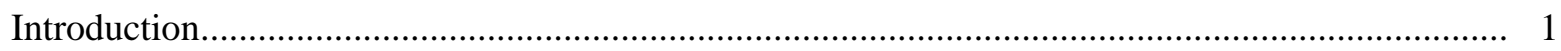

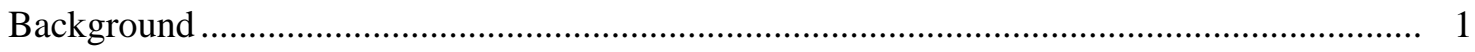

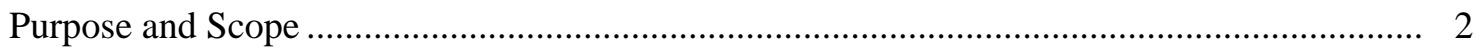

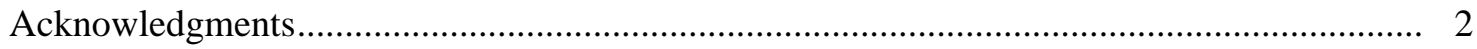

Methods of Data Collection.................................................................................................. 2

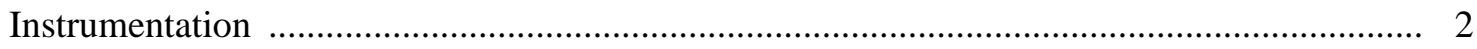

Calibration of Laboratory Instruments........................................................................ 5

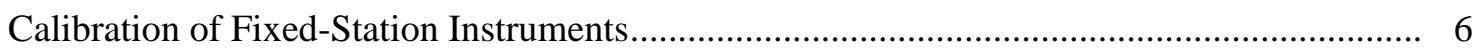

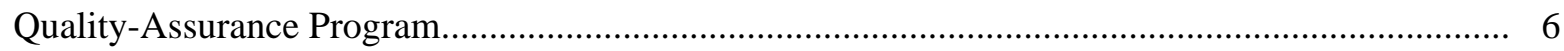

Correcting Real-Time Data for Bias .......................................................................... 7

Review of Hourly and Daily Summary Data ........................................................................ 7

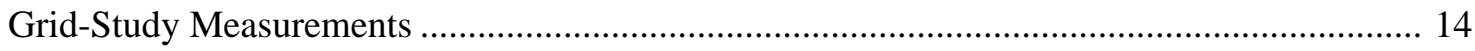

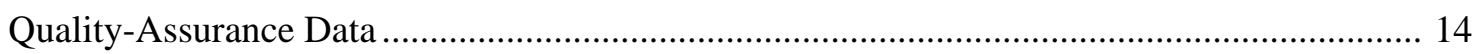

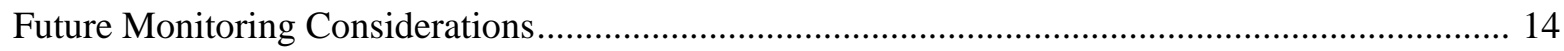

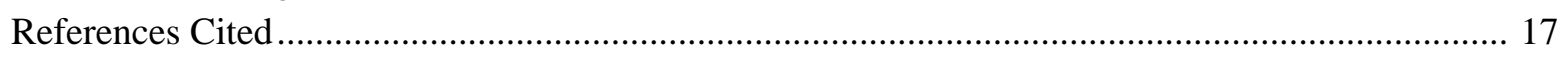

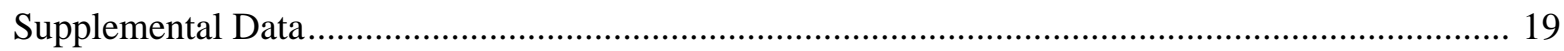




\section{FIGURES}

1. Total dissolved gas fixed stations, lower Columbia River, Oregon and Washington, 1996...................... 3

2. Dissolved oxygen in the Columbia River at John Day forebay, Washington, July - September 1996. ........ 10

3. Dissolved oxygen in the Columbia River, left bank, near Dodson, Oregon (Warrendale fixed station), July 1996.

4. Dissolved oxygen during a period of less than 2 hours (points A and B) in the Columbia River, left bank, near Dodson, Oregon (Warrendale fixed station), July 1996.

5. Hourly values of (A) water temperature, (B) barometric pressure, and (C, D) total dissolved gas at the Columbia River at John Day Dam forebay, Washington, April - September 1996.

6. Hourly values of (A) water temperature, (B) barometric pressure, and (C, D) total dissolved gas at the Columbia River, right bank, near Cliffs, Washington, March - September 1996.

7. Hourly values of (A) water temperature, (B) barometric pressure, and (C, D) total dissolved gas at the Columbia River at The Dalles Dam forebay, Washington, April - September 1996

8. Hourly values of (A) water temperature, (B) barometric pressure, and (C, D) total dissolved gas at the Columbia River, left bank, near The Dalles, Oregon, March - July 1996.

9. Hourly values of (A) water temperature, (B) barometric pressure, and (C, D) total dissolved gas at the Columbia River at The Dalles, Oregon, June - September 1996.

10. Hourly values of (A) water temperature, (B) barometric pressure, and (C, D) total dissolved gas at the Columbia River at Bonneville Dam forebay, Washington, March - September 1996.

11. Hourly values of (A) water temperature, (B) barometric pressure, and (C, D) total dissolved gas at the Columbia River, right bank, near Skamania, Washington, May - September 1996.

12. Hourly values of (A) water temperature, (B) barometric pressure, and (C, D) total dissolved gas at the Columbia River, left bank, near Dodson, Oregon, March - September 1996.

13. Hourly values of (A) water temperature, (B) barometric pressure, and (C, D) total dissolved gas at the Columbia River, right bank, near Washougal, Washington, March - September 1996.

14. Hourly values of (A) water temperature, (B) barometric pressure, and (C, D) total dissolved gas at the Columbia River, right bank, near Kalama, Washington, May - September 1996

15. Hourly values of (A) water temperature, (B) barometric pressure, and (C, D) total dissolved gas at the Columbia River, left bank, at Wauna, Oregon, March - September 1996.

\section{TABLES}

1. Total dissolved gas fixed stations, lower Columbia River, Oregon and Washington, 1996

2. Procedures used to review water temperature, barometric pressure, total dissolved gas, and dissolved oxygen data from fixed stations, lower Columbia River, 1996

3. Summary of hourly water temperature, barometric pressure, and total dissolved gas data lost due to flood damage, equipment problems, and the final review process

4. Comparison of total dissolved gas (TDG) concentrations measured in transects at fixed stations, lower Columbia River, Oregon and Washington, 1996.

5. Daily summary of water temperature for the Columbia River at John Day Dam forebay, Washington, April - September 1996

6. Daily summary of barometric pressure for the Columbia River at John Day Dam forebay, Washington, April - September 1996

7. Daily summary of total dissolved gas, in millimeters of mercury, for the Columbia River at John Day Dam forebay, Washington, April - September 1996.

8. Daily summary of total dissolved gas, in percent saturation, for the Columbia River at John Day Dam forebay, Washington, April - September 1996.

9. Daily summary of water temperature for the Columbia River, right bank, near Cliffs, Washington, March - September 1996 
10. Daily summary of barometric pressure for the Columbia River, right bank, near Cliffs, Washington, March - September 1996

11. Daily summary of total dissolved gas, in millimeters of mercury, for the Columbia River, right bank, near Cliffs, Washington, March - September 1996.

12. Daily summary of total dissolved gas, in percent saturation, for the Columbia River, right bank, near Cliffs, Washington, March - September 1996.

13. Daily summary of water temperature for the Columbia River at The Dalles Dam forebay, Washington, April - September 1996.

14. Daily summary of barometric pressure for the Columbia River at The Dalles Dam forebay, Washington, April - September, 1996.

15. Daily summary of total dissolved gas, in millimeters of mercury, for the Columbia River at The Dalles Dam forebay, Washington, April - September 1996

16. Daily summary of total dissolved gas, in percent saturation, for the Columbia River at The Dalles Dam forebay, Washington, April - September 1996.

17. Daily summary of water temperature for the Columbia River, left bank, near The Dalles, Oregon, March - July 1996

18. Daily summary of barometric pressure for the Columbia River, left bank, near The Dalles, Oregon, March - July 1996

19. Daily summary of total dissolved gas, in millimeters of mercury, for the Columbia River, left bank, near The Dalles, Oregon, March - July 1996

20. Daily summary of total dissolved gas, in percent saturation, for the Columbia River, left bank, near The Dalles, Oregon, March - July 1996

21. Daily summary of water temperature for the Columbia River at The Dalles, Oregon, June September 1996

22. Daily summary of barometric pressure for the Columbia River at The Dalles, Oregon, June September 1996

23. Daily summary of total dissolved gas, in millimeters of mercury, for the Columbia River at The Dalles, Oregon, June - September 1996

24. Daily summary of total dissolved gas, in percent saturation, for the Columbia River at The Dalles, Oregon, June - September 1996

25. Daily summary of water temperature for the Columbia River at Bonneville Dam forebay,

Washington, March - September 1996.

26. Daily summary of barometric pressure for the Columbia River at Bonneville Dam forebay, Washington, March - September 1996.

27. Daily summary of total dissolved gas, in millimeters of mercury, for the Columbia River at Bonneville Dam forebay, Washington, March - September 1996

28. Daily summary of total dissolved gas, in percent saturation, for the Columbia River at Bonneville Dam forebay, Washington, March - September 1996

29. Daily summary of water temperature for the Columbia River, right bank, near Skamania, Washington, March - September 1996

30. Daily summary of barometric pressure for the Columbia River, right bank, near Skamania, Washington, March - September 1996

31. Daily summary of total dissolved gas, in millimeters of mercury, for the Columbia River, right bank, near Skamania, Washington, March - September 1996

32. Daily summary of total dissolved gas, in percent saturation, for the Columbia River, right bank, near Skamania, Washington, March - September 1996

33. Daily summary of water temperature for the Columbia River, left bank, near Dodson, Oregon, March - September 1996

34. Daily summary of barometric pressure for the Columbia River, left bank, near Dodson, Oregon, March - September 1996 
35. Daily summary of total dissolved gas, in millimeters of mercury, for the Columbia River, left bank, near Dodson, Oregon, March - September 1996

36. Daily summary of total dissolved gas, in percent saturation, for the Columbia River, left bank, near Dodson, Oregon, March - September 1996.

37. Daily summary of water temperature for the Columbia River, right bank, near Washougal, Washington, March - September $1996 .$.

38. Daily summary of barometric pressure for the Columbia River, right bank, near Washougal, Washington, March - September 1996......

39. Daily summary of total dissolved gas, in millimeters of mercury, for the Columbia River, right bank, near Washougal, Washington, March - September 1996.

40. Daily summary of total dissolved gas, in percent saturation, for the Columbia River, right bank, near Washougal, Washington, March - September 1996.

41. Daily summary of water temperature for the Columbia River, right bank, near Kalama, Washington, April - September 1996

42. Daily summary of barometric pressure for the Columbia River, right bank, near Kalama, Washington, April - September 1996

43. Daily summary of total dissolved gas, in millimeters of mercury, for the Columbia River, right bank, near Kalama, Washington, April - September 1996.

44. Daily summary of total dissolved gas, in percent saturation, for the Columbia River, right bank, near Kalama, Washington, April - September 1996

45. Daily summary of water temperature for the Columbia River, left bank, at Wauna, Oregon, March - September 1996

46. Daily summary of barometric pressure for the Columbia River, left bank, at Wauna, Oregon, March - September 1996

47. Daily summary of total dissolved gas, in millimeters of mercury, for the Columbia River, left bank, at Wauna, Oregon, March - September 1996

48. Daily summary of total dissolved gas, in percent saturation, for the Columbia River, left bank, at Wauna, Oregon, March - September 1996 


\title{
Total dissolved gas, barometric pressure, and water temperature data, lower Columbia River, Oregon and Washington, 1996
}

\author{
By Dwight Q. Tanner, Howard E. Harrison, and Stuart W. McKenzie
}

\section{Abstract}

Increased levels of total dissolved gas pressure can cause gas-bubble trauma in fish downstream from dams on the Columbia River. In cooperation with the U.S. Army Corps of Engineers, the U.S. Geological Survey collected data on total dissolved gas pressure, barometric pressure, water temperature, and dissolved oxygen pressure at 11 stations on the lower Columbia River from the John Day forebay (river mile 215.6) to Wauna Mill (river mile 41.9) from March to September 1996. Methods of data collection, review, and processing are described in this report. Summaries of daily minimum, maximum, and mean hourly values are presented for total dissolved gas pressure, barometric pressure, and water temperature. Hourly values for these parameters are presented graphically. Dissolved oxygen data are not presented in this report because the quality-control data show that the data have poor precision and high bias. Suggested changes to monitoring procedures for future studies include (1) improved calibration procedures for total dissolved gas and dissolved oxygen to better define accuracy at elevated levels of supersaturation, and (2) equipping dissolved oxygen sensors with stirrers because river velocities at the shoreline monitoring stations probably cannot maintain an adequate flow of water across the membrane surface of the dissolved oxygen sensor.

\section{INTRODUCTION}

The U.S. Army Corps of Engineers (USACE) operates several dams in the Columbia River Basin, which encompasses 259,000 square miles in the Pacific Northwest. These dams are multipurpose facilities that fill regional needs for flood control, navigation, irrigation, recreation, hydropower production, fish and wildlife habitat, water-quality maintenance, and municipal and industrial water supply. When water is released over the spillways of these dams, air is entrained in the water, sometimes increasing the concentration of total dissolved gas (TDG) downstream from the spillways in excess of the U.S. Environmental Protection Agency's water-quality criterion for the protection of freshwater aquatic life of 110-percent saturation. Concentrations above this criterion have been shown to cause gas-bubble trauma in fish and affect other aquatic organisms (U.S. Environmental Protection Agency, 1986). The dams also impede the passage of juvenile and adult migratory fish in the basin. In the past, the USACE minimized spill and regulated streamflow in the region to minimize the production of excess TDG downstream from its dams (Faith Ruffing, U.S. Army Corps of Engineers, written commun., 1996). The USACE collects real-time TDG data (data available within about 4 hours of current time) upstream and downstream from the dams in a network of fixed-station monitors.

\section{Background}

Real-time TDG data are vital to the USACE for dam operation and for monitoring compliance with environmental regulations. The data are used by water managers to maintain water-quality conditions that facilitate fish passage and survival in the lower Columbia River. The U.S. Geological 
Survey (USGS), in a cooperative agreement with the Portland District of the USACE, collected TDG and related data at 11 fixed stations on the lower Columbia River from March through September of 1996 (table 1, fig.1).

To provide a suitable data set for water managers to model TDG in the lower Columbia River, the real-time hourly data were corrected to reflect measurements and (or) changes made during the biweekly calibration visits in 1996 . Preliminary corrections were applied to the data during the monitoring period. After a detailed review of calibration and ancillary data in October and November 1996, final corrections were applied to the 1996 data. The reviewed and corrected hourly data are stored in a USGS data basedata base (Automated Data Processing SystemADAPS) and in a USACE data base.

\section{Purpose and Scope}

The purpose of this study was to provide the USACE with (1) real-time data for managing streamflows and TDG levels upstream and downstream from its project dams in the lower Columbia River, and (2) reviewed and corrected TDG data to evaluate conditions in relation to water-quality criteria and to develop a TDG data base for modeling the effect of various management scenarios of streamflow and spill on TDG levels.

This report describes the data-collection techniques and the quality-assurance program used in the lower Columbia River TDG monitoring program. Eleven fixed stations were located on the Columbia River from the forebay of the John Day Dam (river mile [RM] 215.6) to Wauna Mill (RM 41.9). Daily summary statistics and graphs of the hourly values of water temperature, barometric pressure, and TDG pressure, are presented in the Supplemental Data section of this report for each fixed station. TDG is reported in units of millimeters of mercury ( $\mathrm{mm} \mathrm{Hg}$ ), and in percent saturation (TDG in $\mathrm{mm} \mathrm{Hg}$ divided by barometric pressure in $\mathrm{mm} \mathrm{Hg}$ multiplied by 100).

\section{Acknowledgments}

We wish to acknowledge the aid and funding support of the U.S. Army Corps of Engineers. Our special thanks to Faith E. Ruffing (USACE) for assistance in collecting and compiling the grid-study data and for obtaining equipment and supplies for the monitoring network. The authors also wish to acknowledge Chris J. Brugato for his assistance in data collection and the aid of Jennifer L. Morace (USGS) for helpful suggestions and her special efforts in preparing the manuscript.

\section{METHODS OF DATA COLLECTION}

\section{Instrumentation}

Instrumentation at the fixed stations consisted of a TDG meter and probe, a data-collection platform (DCP), and a power supply. The TDG meters, Model TBO-L, were manufactured by Common Sensing, Inc. The probe had individual sensors for TDG, dissolved oxygen (DO), and temperature. The TDG sensor consisted of a cylindrical framework wound with a length of Silastic (dimethyl silicon) tubing. The tubing was tied off at one end and the other end was connected to a pressure transducer. After the TDG pressure in the river equilibrated with the gas pressure inside the tubing (about 15 to 20 minutes), the pressure transducer produced a repeatable measure of the TDG pressure in the river. The galvanic DO sensor was equipped with a silver anode and a zinc cathode. The water temperature sensor was a thermocouple.

The TDG probe was connected by a weatherproof cable to the TDG meter, which was contained in a plastic enclosure. The meter was equipped with a display panel and keypad for on-site query and calibration. The monitor displayed TDG pressure, in $\mathrm{mm} \mathrm{Hg}$ or percent saturation; DO pressure, in $\mathrm{mm} \mathrm{Hg}$ or percent saturation; water temperature, in degrees Celsius $\left({ }^{\circ} \mathrm{C}\right)$; and barometric pressure, in $\mathrm{mm} \mathrm{Hg}$. The DO sensors were not equipped with stirrers, so they did not facilitate DO measurements in slow-moving water. The transducer for barometric pressure was contained in the TDG meter. 


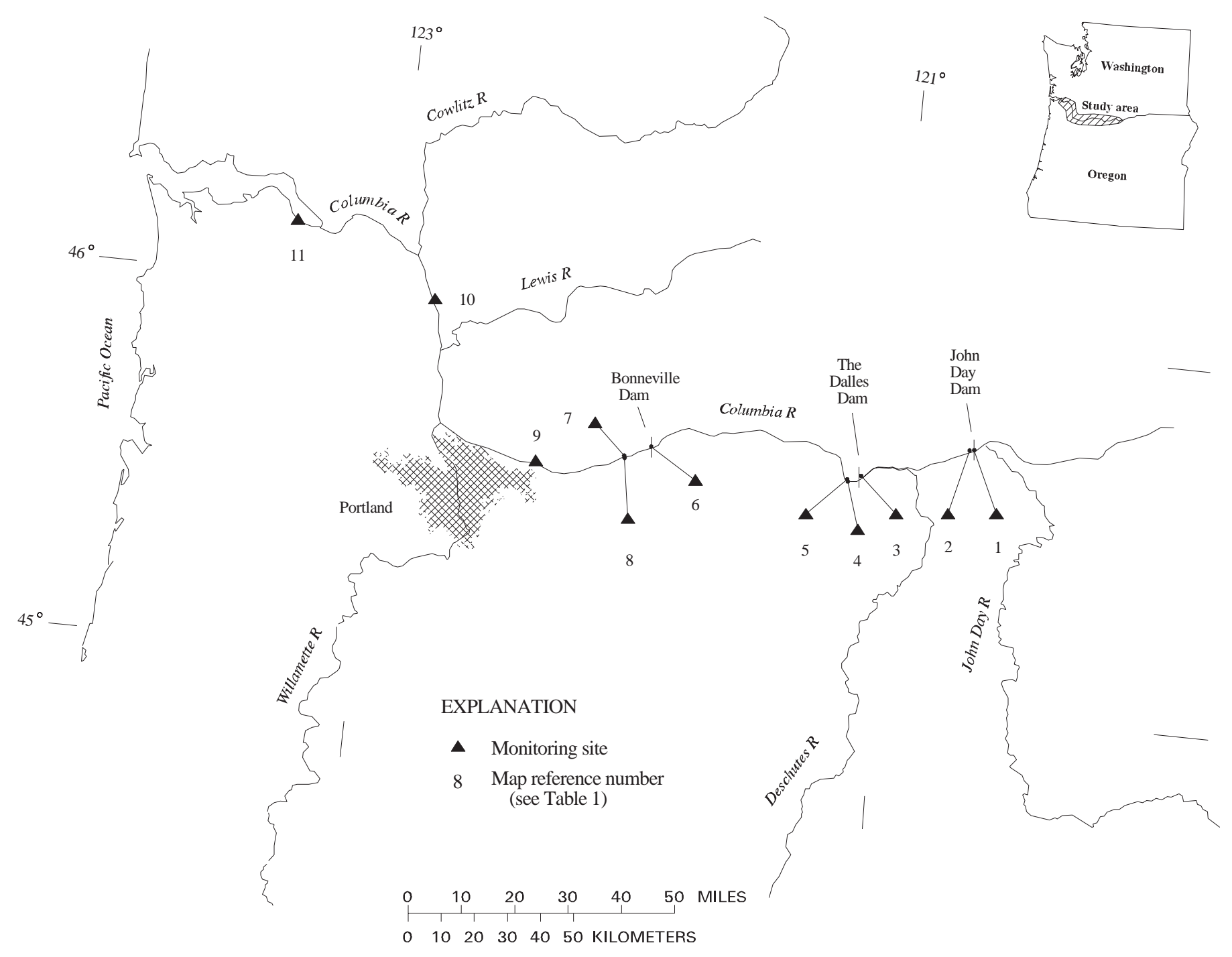

Figure 1. Total dissolved gas fixed stations, lower Columbia River, Oregon and Washington, 1996. 
Table 1. Total dissolved gas fixed stations, lower Columbia River, Oregon and Washington, 1996

[Map reference number refers to figure 1; USACE, U.S. Army Corps of Engineers; Columbia River mile locations were determined from U.S. Geological Suvey (USGS) 7.5-minute topographic maps; stations are referenced by their abbreviated name in this report]

\begin{tabular}{|c|c|c|c|c|c|c|c|}
\hline $\begin{array}{c}\text { Map } \\
\text { reference } \\
\text { number }\end{array}$ & $\begin{array}{l}\text { USACE } \\
\text { site } \\
\text { identifier }\end{array}$ & $\begin{array}{l}\text { Columbia } \\
\text { River mile }\end{array}$ & $\begin{array}{l}\text { USGS } \\
\text { station number }\end{array}$ & $\begin{array}{l}\text { USGS station name } \\
\text { (abbreviated station name) }\end{array}$ & Latitude & Longitude & Period of record \\
\hline 1 & JDA & 215.6 & 454257120413000 & $\begin{array}{l}\text { Columbia River at John Day Dam forebay, Washington } \\
\text { (John Day forebay) }\end{array}$ & $45^{\circ} 42^{\prime} 57^{\prime \prime}$ & $120^{\circ} 41^{\prime} 30^{\prime \prime}$ & April 2 - September 17 \\
\hline 2 & JHAW & 214.7 & 454249120423500 & $\begin{array}{l}\text { Columbia River, right bank, near Cliffs, Washington } \\
\text { (John Day tailwater) }\end{array}$ & $45^{\circ} 42^{\prime} 49^{\prime \prime}$ & $120^{\circ} 42^{\prime} 35^{\prime \prime}$ & March 30 - September 17 \\
\hline 3 & TDA & 192.6 & 453712121071200 & $\begin{array}{l}\text { Columbia River at The Dalles Dam forebay, Washington } \\
\text { (The Dalles forebay) }\end{array}$ & $45^{\circ} 37^{\prime} 12^{\prime \prime}$ & $121^{\circ} 07^{\prime} 12^{\prime \prime}$ & April 12 - September 18 \\
\hline 4 & TDTO & 189.9 & 453606121101000 & $\begin{array}{l}\text { Columbia River, left bank, near The Dalles, Oregon } \\
\text { (The Dalles tailwater) }\end{array}$ & $45^{\circ} 36^{\prime} 06^{\prime \prime}$ & $121^{\circ} 10^{\prime} 10^{\prime \prime}$ & March 29 - July 7 \\
\hline 5 & TDDO & 188.9 & 14105700 & $\begin{array}{l}\text { Columbia River at The Dalles, Oregon } \\
\text { (The Dalles downstream) }\end{array}$ & $45^{\circ} 36^{\prime} 27^{\prime \prime}$ & $121^{\circ} 10^{\prime} 20^{\prime \prime}$ & June 22 - September 18 \\
\hline 6 & BON & 146.1 & 453845121562000 & $\begin{array}{l}\text { Columbia River at Bonneville Dam forebay, Washington } \\
\text { (Bonneville forebay) }\end{array}$ & $45^{\circ} 38^{\prime} 45^{\prime \prime}$ & $121^{\circ} 56^{\prime} 20^{\prime \prime}$ & March 5 - September 16 \\
\hline 7 & SKAW & 140.5 & 453651122022200 & $\begin{array}{l}\text { Columbia River, right bank, near Skamania, Washington } \\
\text { (Skamania) }\end{array}$ & $45^{\circ} 36^{\prime} 51^{\prime \prime}$ & $122^{\circ} 02^{\prime} 22^{\prime \prime}$ & May 4 - September 15 \\
\hline 8 & WRNO & 140.4 & 453630122021400 & $\begin{array}{l}\text { Columbia River, left bank, near Dodson, Oregon } \\
\text { (Warrendale) }\end{array}$ & $45^{\circ} 36^{\prime} 30^{\prime \prime}$ & $122^{\circ} 02^{\prime} 14^{\prime \prime}$ & March 8 - September 16 \\
\hline 9 & CWMW & 121.7 & 453439122223900 & $\begin{array}{l}\text { Columbia River, right bank, at Washougal, Washington } \\
\text { (Camas) }\end{array}$ & $45^{\circ} 34^{\prime} 39^{\prime \prime}$ & $122^{\circ} 22^{\prime} 39^{\prime \prime}$ & March 9 - September 19 \\
\hline 10 & KLAW & 76.9 & 455903122500000 & $\begin{array}{l}\text { Columbia River, right bank, near Kalama, Washington } \\
\text { (Kalama) }\end{array}$ & $45^{\circ} 59^{\prime} 03^{\prime \prime}$ & $122^{\circ} 50^{\prime} 00^{\prime \prime}$ & May 30 - September 15 \\
\hline 11 & WANO & 41.9 & 460923123235800 & $\begin{array}{l}\text { Columbia River, left bank, at Wauna, Oregon } \\
\text { (Wauna Mill) }\end{array}$ & $46^{\circ} 09^{\prime} 23^{\prime \prime}$ & $123^{\circ} 23^{\prime} 58^{\prime \prime}$ & March 7 - September 19 \\
\hline
\end{tabular}


The TDG meter was connected to a Sutron Model 8200 DCP. The DCP had three basic functions: sensor interfacing, data storage, and data transmission to the Geostationary Operational Environmental Satellite (GOES) system (Jones and others, 1991). A crossed Yagi antenna was connected to the DCP using a coaxial cable. The antenna was mounted on a mast to provide transmission to the GOES system.

The TDG meter and the DCP were powered by a 12 -volt gelled-electrolyte battery. The battery was charged by a regulated-voltage circuit from a solar panel or a 120-volt alternating-current line.

The DCP was programmed to record and transmit five parameters: barometric pressure (in $\mathrm{mm} \mathrm{Hg}$ ), TDG pressure (in $\mathrm{mm} \mathrm{Hg}$ ), DO pressure (in $\mathrm{mm} \mathrm{Hg}$ ), water temperature (in ${ }^{\circ} \mathrm{C}$ ), and battery voltage (in volts). Battery-voltage data were monitored to determine whether the instrumentation was receiving adequate power during the monitoring period. The data for each parameter were logged electronically every hour, on the hour, and stored in the DCP memory. Every 4 hours, the DCP transmitted the most recent 12 hours of logged data to the GOES satellite. Consequently, each piece of data was transmitted three times to protect against data loss. The GOES satellite retransmitted the data to a direct readout ground station, where the uncorrected data were automatically decoded and transferred to the USACE data base (Columbia River Operation Hydromet Management System-CHROMS), and to the USGS ADAPS data base. During the biweekly fixed-station calibration visits, the DCP-stored data were downloaded to a laptop personal computer. These data were loaded into the computer system at the USGS office in Portland, Oregon, and were used to fill in any real-time data lost during satellite transmission.

\section{Calibration of Laboratory Instruments}

Two portable instruments were used to periodically calibrate the TDG meters at the fixed stations: (1) a portable "lab-calibrated" TDG meter (model TBO-L by Common Sensing, Inc.) was used for calibrating TDG pressure, DO pressure, and water temperature, and (2) a hand-held aneroid barometer was used as a "reference barometer" for calibrating barometric pressure.

The lab-calibrated TDG meter was calibrated in the USGS laboratory in Portland every 2 weeks, just prior to the fixed-station visits. To calibrate the lab-calibrated meter at the low end of the TDG scale (at atmospheric pressure), the TDG sensor was air dried and the zero-adjustment screw for TDG pressure was adjusted to equal the atmospheric-pressure reading on the reference barometer. The upper end of the TDG scale was calibrated by the manufacturer at the beginning and middle of the monitoring period by disconnecting the Silastic membrane from the probe and applying a known pressure to the transducer in the TDG sensor. On July 23, 1996, during the middle of the monitoring period, the manufacturer's adjustment was only $3 \mathrm{~mm} \mathrm{Hg}$ at a TDG pressure of $1000 \mathrm{~mm} \mathrm{Hg}$.

DO pressure on the lab-calibrated meter was calibrated using a container of air-saturated water. The water at room temperature was saturated with DO using an aquarium aerator and pump. The probe was placed in the air-saturated water to read DO pressure, in $\mathrm{mm} \mathrm{Hg}$. This pressure was recorded, and the meter was switched to display DO in percent saturation. The meter was adjusted to read 100-percent saturation using the vernier calibration knob for DO. The lower end of the DO scale was checked periodically using a sodium sulfite solution, which resulted in readings near $0 \mathrm{~mm} \mathrm{Hg}$.

Temperature was calibrated periodically using a mercury thermometer with certification that was traceable to the National Bureau of Standards (NBS). The probe of the lab-calibrated meter and the mercury thermometer were placed together in water baths of about $4^{\circ} \mathrm{C}$ and $20^{\circ} \mathrm{C}$, and adjustments were made to the zero- and span-temperature screws (respectively) on the lab-calibrated meter to correspond with the readings on the mercury thermometer. Upon receiving manufacturer's recommendations in July 1996 when the river temperature was approaching $20^{\circ} \mathrm{C}$, the procedure was changed so that only the span was adjusted in the $20^{\circ} \mathrm{C}$ water bath. The low end of the scale was checked electronically by the manufacturer. 
The reference barometer was calibrated periodically at the Troutdale airport early in the study and at Portland's National Weather Service later in the study by adjusting the reference barometer to read the barometric pressure reported at these locations.

\section{Calibration of Fixed-Station Instruments}

At about 2-week intervals, the fixed stations were visited and the lab-calibrated meter and the reference barometer were used to calibrate the fixed-station TDG meters. At this time, the DCPs also were checked and serviced.

Upon arrival at the fixed station, a digital multimeter was used to check and record the direct-current battery voltage powering the DCP. The battery-charging system was checked by measuring the voltage either from the alternating-current line or from the direct-current solar panel. A laptop computer was connected to the DCP to view and adjust DCP functions. The electronic clock in the DCP was checked against a wristwatch that had been set to exact atomic time using the computer system at the USGS office in Portland, Oregon. If the DCP clock was off by more than 2 seconds, it was reset. The DCPs operational status and the battery voltage (minimum and maximum) also were checked. The angle and direction of the DCP-transmitting antenna were checked and adjusted if necessary.

The current fixed-station display readings for barometric pressure, TDG pressure, DO pressure, and water temperature were compared to the current DCP readings to ensure agreement between the two units. If the difference between readings for any parameter was significant, the DCP reading was adjusted to equal the reading from the TDG meter. Regardless of whether the DCP reading was adjusted, the difference was noted and considered later in making the final data shift.

The barometric pressure measured by the fixed-station TDG meter was checked against the pressure measured by the reference barometer. If the difference was greater than or equal to $\pm 4 \mathrm{~mm}$ $\mathrm{Hg}$, the fixed-station TDG meter was adjusted to equal the reference barometer.
The fixed-station TDG probe was removed from the river, and the sensors were rinsed with tapwater. The TDG membrane was replaced if there were water droplets within the tubing. The DO membrane and electrolyte were replaced if (1) the membrane was ruptured, (2) bubbles or precipitate appeared beneath the membrane, or (3) the DO cathode was colored. The DO cathode was cleaned lightly with emery paper if it was corroded. The TDG sensor was allowed to dry in the ambient air, which usually took about 15 to 20 minutes. If the TDG in percent saturation was less than 99.5 or more than 100.5 , the zero adjustment for TDG was adjusted to 100.0 percent.

The probes from the fixed-station and lab-calibrated meters were placed in the river at a depth of about 15 feet (ft). The probes were allowed to equilibrate in the river water until the TDG reading on both meters stabilized and changed less than $\pm 1 \mathrm{~mm} \mathrm{Hg}$ in a 2-minute interval. The equilibration period was usually less than 25 minutes. The TDG, DO, and temperature readings from the fixed-station meter were compared to the readings from the lab-calibrated meter. If the difference was greater than $14 \mathrm{~mm} \mathrm{Hg}$ for TDG, $5 \mathrm{~mm} \mathrm{Hg}$ for DO, or $0.4^{\circ} \mathrm{C}$ for temperature, the fixed-station meter was adjusted to read the same as the lab-calibrated meter using the span adjustment for the respective parameters. Regardless of whether the fixed-station readings were adjusted in the field, the differences were noted and considered later in making the final data shifts.

\section{QUALITY-ASSURANCE PROGRAM}

A quality-assurance (QA) program was used in this study to determine the accuracy and precision of the TDG, barometric pressure, water temperature, and DO measurements. This section describes how calibration data and ancillary data were used to review and process the hourly data for each parameter. 


\section{Correcting Real-Time Data for Bias}

This section describes methods for processing hourly data (TDG pressure, barometric pressure, and water temperature) for bias. Each parameter has different characteristics with respect to bias and variability. In addition, each river reach has specific characteristics affecting the range of expected values and the daily variation of values.

Initially, the data were screened for gross anomalies. For example, in the lower Columbia River from March through September, TDG generally ranges from about 750 to $1,100 \mathrm{~mm} \mathrm{Hg}$, barometric pressure from about 740 to $775 \mathrm{~mm}$ $\mathrm{Hg}$, and water temperature from about 3 to $23^{\circ} \mathrm{C}$. Values outside of these typical ranges were carefully reviewed for validity. The hourly data for each parameter at a station were graphed by month, which provided sufficient resolution to view diel variability and weekly patterns. For all data except barometric pressure, 1 or 2 hours of data were deleted about every 2 weeks because the probe was removed from the water during the biweekly calibrations.

Bias in the hourly data was removed by shifting the data to agree with the calibration values from the lab-calibrated meter and the reference barometer. A shift also would include any DCP offsets from the fixed-station TDG meter reading that would cause a bias. At the start-up of the monitors in March or April, the first shift applied to all parameters at a station was zero because the TDG meters initially had been set equal to the calibration values. Subsequent shifts or deletions fell into one of three categories:

1. The fixed-station TDG meter was not adjusted at calibration time-For example, assume that on April 26, TDG was checked at a fixed station and the lab-calibrated meter read a TDG pressure of $950 \mathrm{~mm} \mathrm{Hg}$ and the fixed-station meter read $952 \mathrm{~mm} \mathrm{Hg}$. On April 26, the fixed-station meter was not adjusted because the difference was small. On May 10, TDG was checked again at the fixed station, and the lab-calibrated meter read $960 \mathrm{~mm} \mathrm{Hg}$ and the fixed-station meter read $956 \mathrm{~mm} \mathrm{Hg}$. Once again, the fixed-station meter would not have been adjusted because of the small difference.
When processing the hourly data using the ADAPS software, a shift of -2 to $+4 \mathrm{~mm} \mathrm{Hg}$ would be linearly interpolated and applied to the TDG data from April 26 to May 10, respectively.

2. The fixed-station TDG meter was adjusted (usually to agree with the lab-calibrated meter) at the time of calibration-For example, on June 20 at 2:00 pm, assume a lab-calibrated meter read a TDG pressure of $975 \mathrm{~mm} \mathrm{Hg}$ and the fixed-station meter read $1000 \mathrm{~mm} \mathrm{Hg}$. The difference was noted and the fixed-station meter was adjusted to read $975 \mathrm{~mm} \mathrm{Hg}$ (a shift of $-25 \mathrm{~mm} \mathrm{Hg}$ ). When correcting the hourly data, a shift of $-25 \mathrm{~mm}$ $\mathrm{Hg}$ would be input for June 20 at 2:00 pm, which would be linearly interpolated and applied to the previous two weeks of data. A second shift of $0 \mathrm{~mm} \mathrm{Hg}$ would be input at 2:10 pm because the fixed-station-meter reading was adjusted to equal the lab-calibrated-meter reading. This second shift would be applied to the start of the next two weeks of monitoring data.

3. The magnitude of the difference between the fixed-station and the lab-calibrated meters was large; after shifting the hourly data and comparing it with other station data or other ancillary data, the data did not look reasonable-In those instances, the data from that calibration time were deleted back to a time when the data looked reasonable.

\section{Review of Hourly and Daily Summary Data}

To determine the quality of the data and to help identify anomalous values, data-review procedures included a variety of checking procedures that are outlined in table 2. Calibrations of the labcalibrated meter and the reference barometer were reviewed. All fixed-station calibration shifts were checked for the correct magnitude and direction of shifts and for the correct date and time of shift. Values observed at the fixed stations during calibration visits were cross-checked with the values received from the GOES system during that same time period. 
Table 2. Procedures used to review water temperature, barometric pressure, total dissolved gas, and dissolved oxygen data from fixed stations, lower Columbia River, 1996

[See table 1 for full station names and locations]

\section{Water temperature}

Compared solar-radiation data from Hood River to water temperature data from the Bonneville forebay fixed station.

- Verified that the direction of change in solar radiation corresponded to the direction of change in water temperature.

Compared air-temperature data from the National Weather Service (data recorded every 3 hours) to hourly water temperature data from the Bonneville forebay fixed station.

- Verified that the direction of change in air temperature corresponded to the direction of change in water temperature.

Compared water temperature at the Bonneville forebay fixed station to water temperatures at all fixed stations.

- Data were deleted when the absolute value of the differences in daily mean water temperatures (Bonneville forebay's temperature minus another station's temperature) exceeded those values listed below. Few data values were deleted in this step.

\begin{tabular}{lc}
\hline $\begin{array}{c}\text { Station data compared } \\
\text { with Bonneville forebay } \\
\text { fixed-station data }\end{array}$ & $\begin{array}{c}\text { Review criteria, differences } \\
\text { in daily mean temperature } \\
\text { not to be exceeded, } \\
\text { in degrees Celsius }\end{array}$ \\
\hline Wauna Mill & 1.3 \\
Kalama & 1.1 \\
Camas & 1.0 \\
Warrendale & 1.0 \\
Skamania & 1.0 \\
The Dalles stations & 1.0 \\
John Day stations & 1.1 \\
\hline
\end{tabular}

Compared water temperature at Wauna Mill (Columbia River mile 41.9) to instantaneous water temperature measured at the U.S. Geological Survey's water-quality monitoring station at Beaver Army Terminal (Columbia River mile 53.8).

Barometric Pressure

Compared barometric pressure data from the National Weather Service in Portland to pressure data from the Camas fixed station.

- Camas fixed-station data were biased by $+5 \mathrm{~mm} \mathrm{Hg}$, so Camas data were shifted to remove the bias.

Compared barometric pressure data from the other fixed stations to the barometric pressure data from the Camas fixed station.

- After correcting the data for changes in elevation, barometric pressure data from the other stations were determined to be biased by $+5 \mathrm{~mm} \mathrm{Hg}$. Consequently, data from all stations were shifted to remove the bias. In addition to making data comparisons with the Camas station, data from nearby sites were compared among one another to identify data anomalies. For example, barometric pressure data from the John Day forebay were compared to data from the John Day tailwater (data from these two adjacent stations should be nearly identical after correcting for slight changes in elevation).

Compared barometric pressure data from the Wauna Mill fixed station to instantaneous barometric pressure data from the U.S.

Geological Survey's water-quality monitoring station at Beaver Army Terminal (Columbia River mile 53.8).

\section{Total Dissolved Gas}

Compared total dissolved gas (TDG) concentrations versus spill amounts at the Dams.

- Verified that as the rate of spill increased, the TDG concentration generally increased.

\begin{tabular}{l}
\hline \multicolumn{1}{c}{ Comparisons } \\
\hline McNary spill versus TDG at John Day forebay \\
John Day spill versus TDG at John Day tailwater \\
The Dalles spill versus TDG at The Dalles tailwater \\
Bonneville spill versus TDG at all fixed stations downstream from Bonneville Dam \\
\hline
\end{tabular}


Table 2. Procedures used to review water temperature, barometric pressure, total dissolved gas, and dissolved oxygen data from fixed stations, lower Columbia River, 1996-Continued

Total Dissolved Gas-Continued

Other total dissolved gas intersite comparisons:

\begin{tabular}{ll}
\hline TDG Review Criterion & \multicolumn{1}{c}{ Intersite Comparisons of TDG Data } \\
\hline Upstream $\leq$ downstream & John Day forebay versus tailwater \\
Upstream $\geq$ downstream & John Day tailwater versus The Dalles forebay \\
Upstream $\leq$ downstream & The Dalles forebay versus The Dalles tailwater stations \\
Upstream $\geq$ downstream & The Dalles tailwater versus Bonneville forebay \\
Upstream $\leq$ downstream & Bonneville forebay versus Skamania and Warrendale \\
Should be nearly equal & Skamania versus Warrendale \\
Upstream $\geq$ downstream & Skamania and Warrendale versus Camas \\
Upstream $\geq$ downstream & Camas versus Kalama \\
Upstream $\geq$ downstream & Kalama versus Wauna \\
\hline
\end{tabular}

\section{Dissolved Oxygen}

Dissolved oxygen (DO) data were reviewed as listed below. The data had poor precision and a large variable bias probably because the DO sensor was not equipped with a stirrer (stream velocities at the fixed stations are probably inadequate for measuring DO without a DO stirrer) and possibly because of electrical interference. Owing to this high variability in the data, DO values were not published in this report.

Compared DO at Wauna Mill to instantaneous DO measured at the U.S. Geological Survey's water-quality monitoring station at Beaver Army Terminal.

Compared TDG (in percent saturation) to DO (in percent saturation). The two measurements were expected to be similar because biological productivity and respiration are low in the lower Columbia River.

- Intrasite comparisons of TDG and DO reveal large random differences in the hourly data. These differences appear to result from inadequate velocities at the membrane surface of the DO sensor and (or) electrical interference. These constraints hamper an accurate calibration of the fixed-station DO sensor.

Examples of Velocity Effects on DO:

- Hourly DO values at the John Day forebay fixed station exceeded TDG values during the spring and early summer high-flow periods when water velocities were high. During low streamflows and water velocities in the mid to late summer, however, DO values dropped well below the TDG values (fig. 2). During the low flows, DO probably dropped because it was being depleted at the membrane surface of the DO sensor. Stream velocity was inadequate to maintain a fresh supply of DO across the membrane.

-DO concentrations at the Wauna Mill fixed station routinely dropped to about 60-percent saturation during periods of near-zero stream velocities that occurred during slack tide—-typical DO variability within a day exceeded 30- to 40-percent saturation.

Electrical Interference:

-Electrical interference with the galvanic probe or an inadequate ground may be causing the large range in DO variability (in excess of 40-percent saturation) at the Warrendale fixed station (fig. 3).

Problem in Calibrating a Highly Variable DO Monitor:

-Using the July 6 DO data from the Warrendale fixed station as an example (fig. 4), the data values labeled $A$ and $B$ were recorded within a 2-hour period. The variation in DO during this time period spans nearly 40-percent saturation. Under these circumstances, a meter calibrated at time $A$ probably would require a large negative shift; however, a unit calibrated at time $B$ probably would require a large positive shift. In order to obtain accurate DO data, the DO meter and sensor must produce stable readings that are not highly variable over time. This large variability seems to be related to streamflow velocity effects and possibly to electrical interference. 


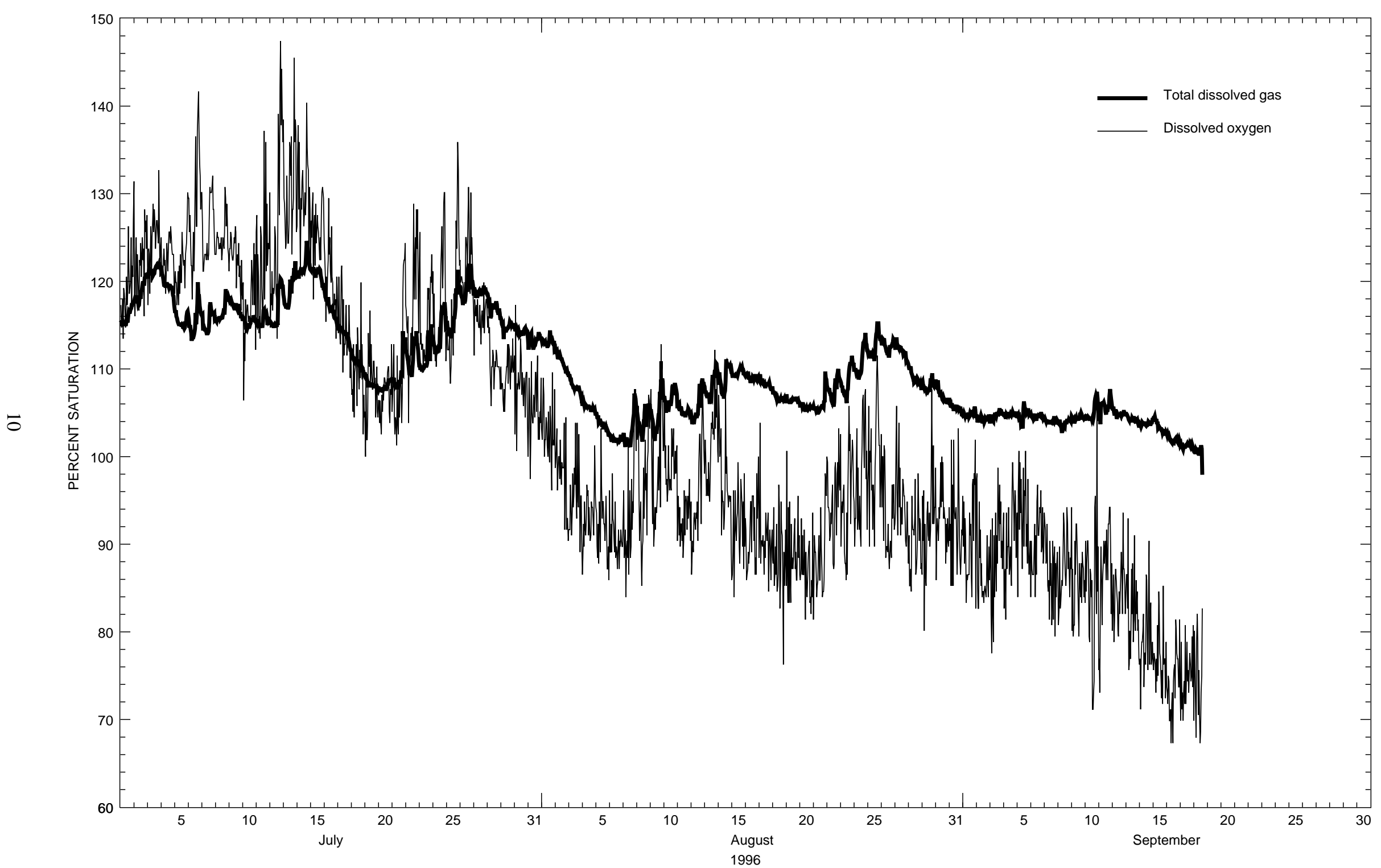

Figure 2. Dissolved oxygen in the Columbia River at John Day forebay, Washington, July - September 1996. 


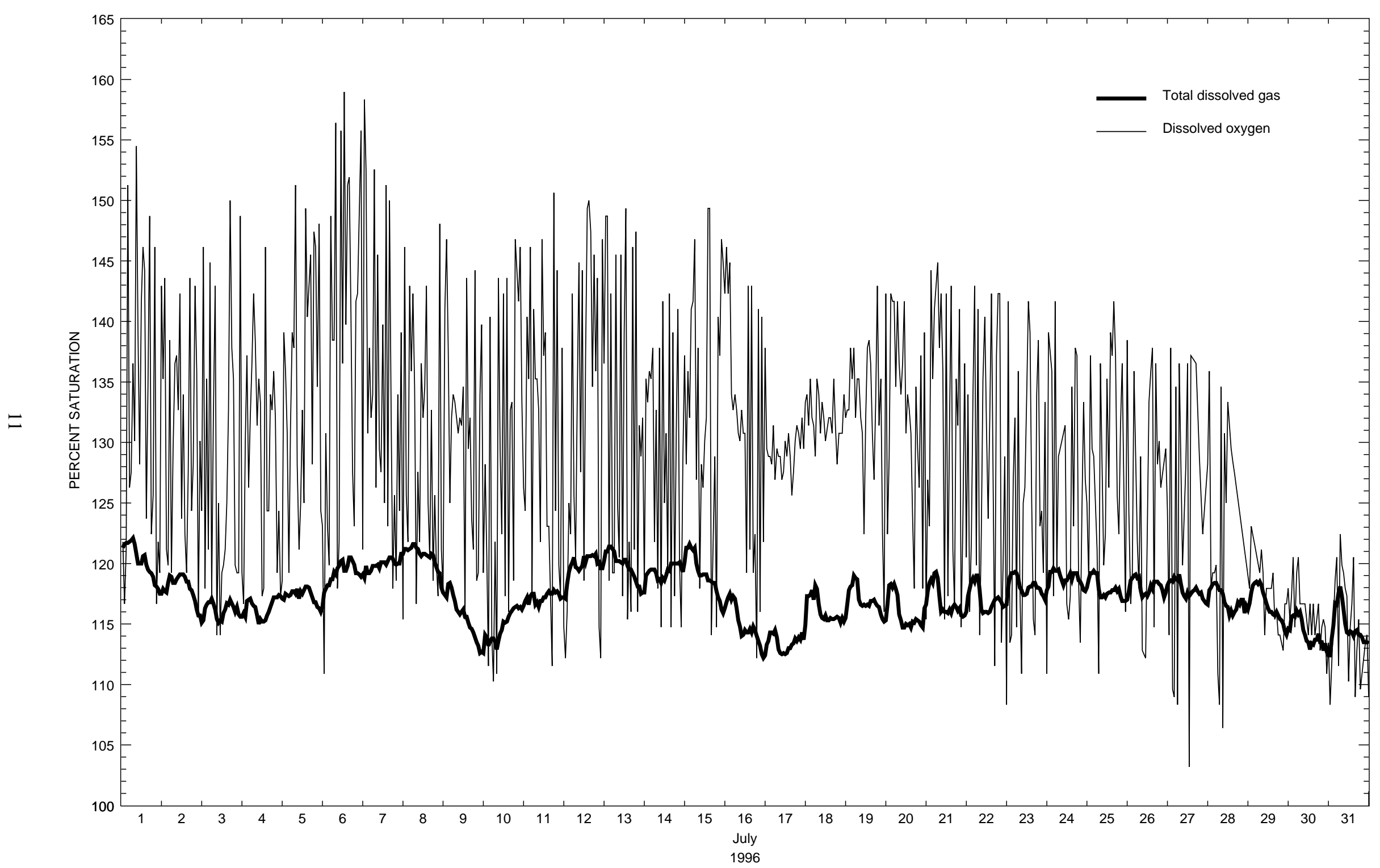

Figure 3. Dissolved oxygen in the Columbia River, left bank, near Dodson, Oregon (Warrendale fixed station), July 1996. 


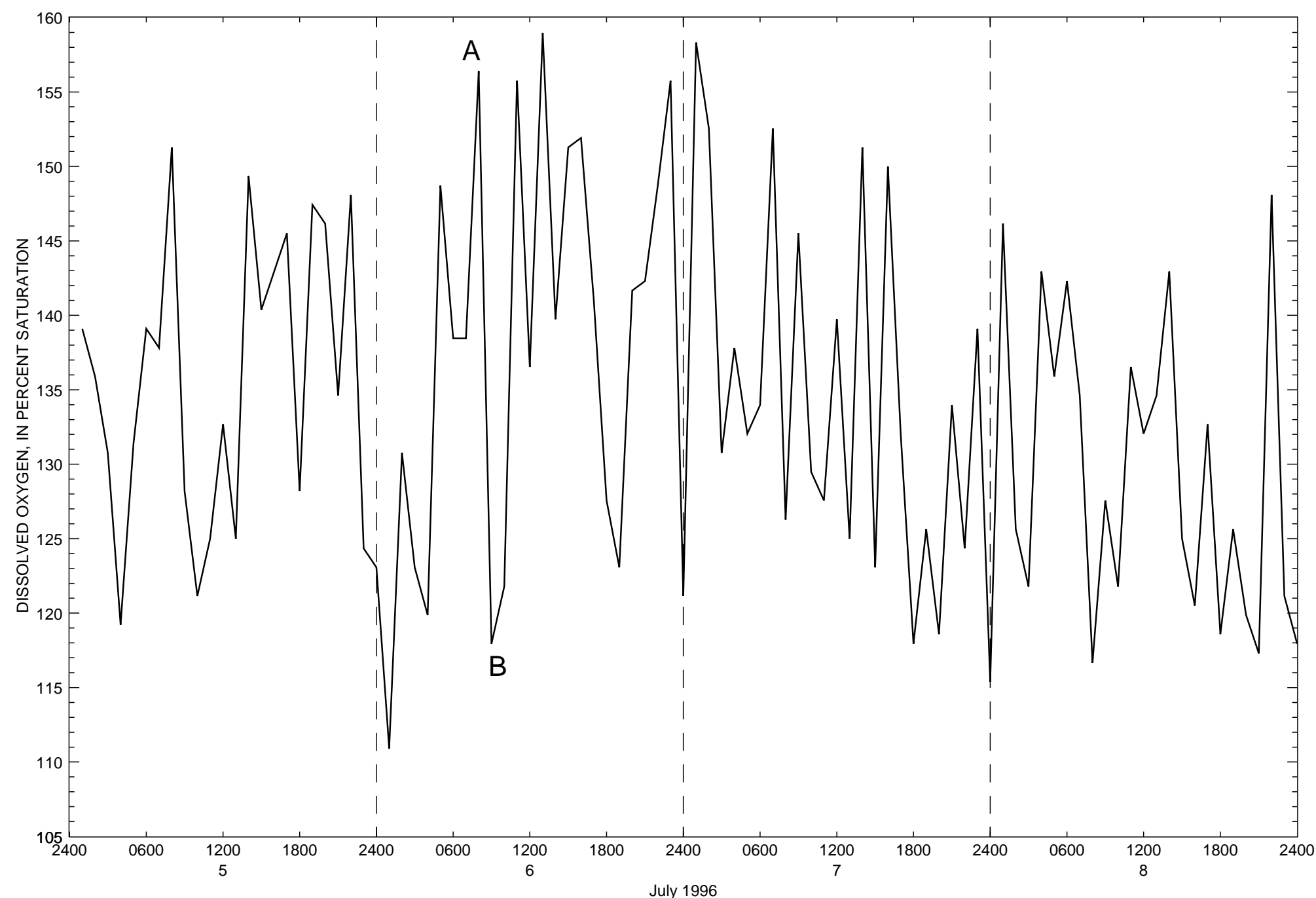

Figure 4. Dissolved oxygen during a period of less than 2 hours (points A and B) in the Columbia River, left bank, near Dodson, Oregon (Warrendale fixed station), July 1996. 
Data processing included review of hourly values and summary statistics, including daily minimum, maximum, and mean values. When anomalous hourly values were observed and verified to be outliers through the analysis of calibration and ancillary data, the values were deleted. If data values were questionable and neither the calibration data nor the ancillary data fully supported data deletion, one or more of the summary statistics were qualified as "estimated."

When fewer than 24 hourly values were available to calculate a daily minimum, maximum, and mean, the hourly values were reviewed to determine whether daily summary statistics could be reported. A daily summary statistic was reported when the diel pattern of the hourly values was representative of the daily variation and suggested that the daily statistical values could be determined. After all shifts were applied, the hourly data were plotted by month to check for continuity and to ensure that the shifts were applied correctly.

Review of the hourly values resulted in the following data qualifications or deletions:

- Dissolved oxygen data were not published because the data had high variability and high bias, which probably resulted from inadequate water velocities across the membrane surface of the DO sensor. Electrical interferences at the Warrendale fixed station also may account for some of the poor precision.

- Water temperature and TDG data from the John Day forebay showed anomalously high readings on several occasions that lasted several days and then returned to more normal levels. These data were not deleted from the data base because they probably represent actual conditions in a parcel of water near the probe (possibly due to wind actions); however, the data may not be representative of conditions throughout the cross section in the forebay.

- TDG data from the Warrendale fixed station may be negatively biased because the probe was often at a depth of less than $10 \mathrm{ft}$. Early in the season, the short probe housing at Warrendale limited probe depth; later in the season, the shallow river depth limited probe depth. This bias can not be corrected because, in addition to depth, degassing is controlled by a variety of factors that were not monitored. For example, the rate of degassing is likely a function of wind speed, water turbulence, and stream velocity; their effects on TDG levels have not been quantified at this station. With a probe at a 10-ft depth, measured TDG levels greater than about 130-percent saturation may be biased low because of degassing. At a depth of $3 \mathrm{ft}$, measured TDG levels greater than about 110-percent saturation may be biased low because of degassing. The probe depths at the Warrendale fixed station were:

$\begin{array}{ll}13 \mathrm{ft} \text { on } 03-07-96, & 3 \mathrm{ft} \text { on } 03-20-96, \\ 13 \mathrm{ft} \text { on } 03-23-96, & 3 \mathrm{ft} \text { on } 04-01-96, \\ 3 \mathrm{ft} \text { on } 04-18-96, & 7.5 \mathrm{ft} \text { on } 06-04-96, \\ 8.6 \mathrm{ft} \text { on } 07-18-96, & \text { and } 5.4 \mathrm{ft} \text { on } 09-17-96 .\end{array}$

On March 7, 1996, TDG levels at 3- and 10-ft depths at the Warrendale fixed station were about 110- and 120-percent saturation, respectively. On May 8, 1996, the TDG levels at 3 - and 13-ft depths were about 119-and 120-percent saturation. Differences observed between the March and May values may have resulted from differences in wind and water velocity along the shoreline at the fixed station on those days. A few TDG measurements made in the flow channel by USGS and USACE personnel at the John Day tailwater and The Dalles tailwater stations in 1996 show TDG differences at the 3-and 15-ft depths that were generally less than 1-percent saturation (Faith Ruffing, U.S. Army Corps of Engineers, written commun., 1996). These flow-channel data, however, may not be representative of differences along the bank at the fixed-station locations. At all other fixed stations, the probes were maintained at depths of $12 \mathrm{ft}$ or greater, except for (1) The Dalles downstream station, which was $10 \mathrm{ft}$ deep or less on calibration visits from 08-15-96 through 09-04-96, and (2) the Wauna Mill station, which was observed to be $10 \mathrm{ft}$ deep on $05-09-96$ and $11.3 \mathrm{ft}$ on 07-12-96, and (3) the Warrendale fixed station as described above. 
After the review process was complete, the amount of missing data were summarized for TDG, barometric pressure, and water temperature (table 3). Overall, 15 percent of the temperature data, 10 percent of the barometric pressure data, and 9 percent of the TDG pressure data were lost as a result of flood damage, equipment failure, and the final review process. The majority of missing data for these parameters resulted from flood damage at the start of the monitoring period.

\section{Grid-Study Measurements}

Grid-study measurements in 1996 were designed to describe the temporal and spatial variability of TDG in the river transects at eight fixed stations during selected high-flow and high-spill conditions (table 4). The data may be used to define the bias between TDG levels measured at the fixed stations and in the river cross sections. Grid-study measurements consisted of minute- by-minute TDG measurements made from a boat in the transect at the fixed station, as well as concurrent measurements by two TDG meters at the fixed-station location. In addition to providing transect information, the study provides data on short-term instrument precision at several of the fixed-station locations (Faith Ruffing, U.S. Army Corps of Engineers, written commun., 1996).

\section{Quality-Assurance Data}

Bias can be defined as a systematic error that is either a positive or negative deviation from the true value. For this study, the true value (calibration standards) for each parameter was as follows: (1) for barometric pressure and TDG levels near $760 \mathrm{~mm} \mathrm{Hg}$, the reference barometer, which was calibrated to barometers at the Troutdale Airport and Portland's National Weather Service, (2) for TDG levels above $760 \mathrm{~mm} \mathrm{Hg}$, the manufacturer's check of the TDG meter's pressure transducer in the lab-calibrated TDG meter, (3) for temperature, comparison checks to a NBS-certified mercury thermometer, and (4) for DO, an air-saturated water bath.
Variability is the degree of random variation in independent, repeated measurements. It includes variation due to measurement error as well as environmental variations. Analytical variability that may be attributed to measurement errors for TDG is about \pm 1 - to 2-percent saturation; for barometric pressure, about $\pm 5 \mathrm{~mm}$ $\mathrm{Hg}$; and, for water temperature, about $\pm 1^{\circ} \mathrm{C}$. These measurement errors are small when compared to the large changes that were observed for these parameters in the lower Columbia River from March through September of 1996.

\section{FUTURE MONITORING CONSIDERATIONS}

Although the amount of effort in calibrating and maintaining the operation of the fixed stations in 1996 was greatly expanded over previous years, the following improvements can be made to further improve the overall quality of the data:

1. Calibration protocols for TDG can be improved to better define the accuracy of the TDG meters at elevated levels of supersaturation, both in the laboratory and at fixed stations. A pressurized chamber could be constructed to calibrate the TDG meters at the levels of supersaturation that are expected in the Columbia River. If a calibration chamber is not constructed, the TDG sensor at each of the fixed stations could be checked more frequently at elevated pressures by removing the Silastic membrane and applying a known pressure directly to the transducer.

2. Calibration protocols also could be improved for DO by supplementing biweekly air calibrations with periodic Winkler titrations to determine precision and to verify accuracy at supersaturated levels.

3. If variations in stream velocity are shown to affect the accurate measurement of DO, then DO probes could be equipped with stirrers. If stirrers are not available, then another DO instrument with a stirrer can be deployed. 
Table 3. Summary of hourly water temperature, barometric pressure, and total dissolved gas data lost due to flood damage, equipment problems, and the final review process

[Planned monitoring hours for stations upstream from Bonneville forebay correspond to the period from April 1 to September 15, 1996; planned hours at and downstream from Bonneville forebay correspond to the period from March 15 to September 15, 1996; see table 1 for full station names and locations]

\begin{tabular}{|c|c|c|c|c|c|c|c|c|c|}
\hline \multirow[t]{2}{*}{$\begin{array}{l}\text { Abbreviated } \\
\text { station name }\end{array}$} & \multirow[t]{2}{*}{$\begin{array}{l}\text { Planned } \\
\text { monitoring, } \\
\text { in hours }\end{array}$} & \multicolumn{2}{|c|}{$\begin{array}{l}\text { Data lost due to } \\
\text { flood damage }\end{array}$} & \multicolumn{2}{|c|}{$\begin{array}{c}\text { Data lost due to } \\
\text { equipment } \\
\text { problems }\end{array}$} & \multicolumn{2}{|c|}{$\begin{array}{l}\text { Data deleted in } \\
\text { the final review } \\
\text { process }\end{array}$} & \multicolumn{2}{|c|}{$\begin{array}{c}\text { Total amount of } \\
\text { data lost or } \\
\text { deleted }\end{array}$} \\
\hline & & Hours & Percent & Hours & Percent & Hours & Percent & Hours & Percent \\
\hline \multicolumn{10}{|c|}{ Water temperature } \\
\hline John Day forebay & 4,008 & 0 & 0 & 0 & 0 & 3 & 0.1 & 3 & 0.1 \\
\hline John Day tailwater & 4,008 & 0 & 0 & 0 & 0 & 6 & 0.1 & 6 & 0.1 \\
\hline The Dalles forebay & 4,008 & 0 & 0 & 375 & 9.4 & 414 & 10 & 789 & 20 \\
\hline The Dalles tailwater & 2,352 & 0 & 0 & 80 & 3.4 & 3 & 0.1 & 83 & 3.5 \\
\hline The Dalles downstream & 2,088 & 0 & 0 & 3 & 0.1 & 325 & 16 & 328 & 16 \\
\hline Bonneville forebay & 4,416 & 0 & 0 & 0 & 0 & 13 & 0.3 & 13 & 0.3 \\
\hline Skamania & 4,416 & 1,200 & 27 & 0 & 0 & 1 & 0 & 1,201 & 27 \\
\hline Warrendale & 4,416 & 121 & 2.7 & 0 & 0 & 181 & 4.1 & 302 & 6.8 \\
\hline Camas & 4,416 & 76 & 1.7 & 20 & 0.4 & 4 & 0.1 & 100 & 2.2 \\
\hline Kalama & 4,416 & 1,824 & 41 & 0 & 0 & 2 & 0 & 1,826 & 41 \\
\hline Wauna Mill & 4,416 & 0 & 0 & 1,739 & 39 & 9 & 0.2 & 1,748 & 39 \\
\hline Subtotals & 42,960 & 3,221 & -- & 2,217 & -- & 961 & -- & 6,399 & -- \\
\hline Total percent of data lost & -- & -- & 7.5 & -- & 5.2 & -- & 2.2 & -- & 15 \\
\hline \multicolumn{10}{|c|}{ Barometric pressure } \\
\hline John Day forebay & 4,008 & 0 & 0 & 0 & 0 & 0 & 0 & 0 & 0 \\
\hline John Day tailwater & 4,008 & 393 & 9.8 & 0 & 0 & 300 & 7.5 & 693 & 17 \\
\hline The Dalles forebay & 4,008 & 0 & 0 & 399 & 9.9 & 16 & 0.4 & 415 & 10 \\
\hline The Dalles tailwater & 2,352 & 0 & 0 & 72 & 3.0 & 6 & 0.3 & 78 & 3.3 \\
\hline The Dalles downstream & 2,088 & 0 & 0 & 3 & 0.1 & 44 & 2.1 & 47 & 2.2 \\
\hline Bonneville forebay & 4,416 & 0 & 0 & 0 & 0 & 2 & 0 & 2 & 0 \\
\hline Skamania & 4,416 & 1,200 & 27 & 0 & 0 & 0 & 0 & 1,200 & 27 \\
\hline Warrendale & 4,416 & 121 & 2.7 & 0 & 0 & 2 & 0 & 123 & 2.8 \\
\hline Camas & 4,416 & 76 & 1.7 & 0 & 0 & 2 & 0 & 78 & 1.7 \\
\hline Kalama & 4,416 & 1,824 & 41 & 2 & 0 & 0 & 0 & 1,826 & 41 \\
\hline Wauna Mill & 4,416 & 0 & 0 & 12 & 0.3 & 9 & 0.2 & 21 & 0.5 \\
\hline Subtotals & 42,960 & 3,614 & -- & 488 & -- & 381 & -- & 4,483 & -- \\
\hline Total percent of data lost & -- & -- & 8.4 & -- & 1.1 & -- & 0.9 & -- & 10 \\
\hline \multicolumn{10}{|c|}{ Total dissolved gas (data recorded in millimeters of mercury) } \\
\hline John Day forebay & 4,008 & 0 & 0 & 0 & 0 & 5 & 0.1 & 5 & 0.1 \\
\hline John Day tailwater & 4,008 & 0 & 0 & 0 & 0 & 13 & 0.3 & 13 & 0.3 \\
\hline The Dalles forebay & 4,008 & 0 & 0 & 435 & 11 & 6 & 0.2 & 441 & 11 \\
\hline The Dalles tailwater & 2,352 & 0 & 0 & 72 & 3.0 & 5 & 0.2 & 77 & 3.3 \\
\hline The Dalles downstream & 2,088 & 0 & 0 & 4 & 0.2 & 9 & 0.4 & 13 & 0.6 \\
\hline Bonneville forebay & 4,416 & 0 & 0 & 0 & 0 & 12 & 0.3 & 12 & 0.3 \\
\hline Skamania & 4,416 & 1,200 & 27 & 0 & 0 & 1 & 0 & 1,201 & 27 \\
\hline Warrendale & 4,416 & 121 & 2.7 & 0 & 0 & 7 & 0.2 & 128 & 2.9 \\
\hline Camas & 4,416 & 76 & 1.7 & 21 & 0.5 & 6 & 0.1 & 103 & 2.3 \\
\hline Kalama & 4,416 & 1,824 & 41 & 0 & 0 & 4 & 0.1 & 1,828 & 41 \\
\hline Wauna Mill & 4,416 & 0 & 0 & 12 & 0.3 & 18 & 0.4 & 30 & 0.7 \\
\hline Subtotals & 42,960 & 3,221 & -- & 544 & -- & 86 & -- & 3,851 & -- \\
\hline Total percent of data lost & -- & -- & 7.5 & -- & 1.3 & -- & 0.2 & -- & 9.0 \\
\hline
\end{tabular}


Table 4. Comparison of total dissolved gas (TDG) concentrations measured in transects at fixed stations, lower Columbia River, Oregon and Washington, 1996

[Positive differences indicate that concentrations along the transect exceed those at the fixed station; vertical-sampling locations within the transect were approximately equally spaced; --, not available; data compiled by Faith E. Ruffing, biologist, U. S. Army Corps of Engineers, 1996]

\begin{tabular}{|c|c|c|c|c|c|c|c|c|}
\hline \multirow{3}{*}{$\begin{array}{c}\text { Amount } \\
\text { of spill, } \\
\text { in percent } \\
\text { of total } \\
\text { streamflow }\end{array}$} & \multirow{3}{*}{$\begin{array}{l}\text { Depth of } \\
\text { measurement, } \\
\text { in feet }\end{array}$} & \multirow{3}{*}{$\begin{array}{l}\text { TDG at fixed } \\
\text { station on } \\
\text { left bank, } \\
\text { in percent } \\
\text { saturation }\end{array}$} & \multicolumn{5}{|c|}{$\begin{array}{l}\text { Difference between TDG concentrations at fixed station } \\
\text { and at location in transect, in percent of saturation }\end{array}$} & \multirow{3}{*}{$\begin{array}{l}\text { TDG at fixec } \\
\text { station on } \\
\text { right bank, } \\
\text { in percent } \\
\text { saturation }\end{array}$} \\
\hline & & & \multicolumn{5}{|c|}{ Sampling location within transect } & \\
\hline & & & $\begin{array}{c}\mathrm{A} \\
\text { (left bank) }\end{array}$ & B & $\mathrm{C}$ & D & $\begin{array}{c}\mathrm{E} \\
\text { (right bank) }\end{array}$ & \\
\hline \multicolumn{9}{|c|}{ John Day tailwater } \\
\hline \multirow[t]{2}{*}{15} & 3 & -- & -- & -0.8 & -0.2 & -0.7 & -0.8 & -- \\
\hline & 15 & -- & -- & -1.1 & 0.2 & 0.0 & 0.6 & 120.0 \\
\hline \multicolumn{9}{|c|}{ The Dalles tailwater } \\
\hline \multirow[t]{2}{*}{33} & 15 & 114.4 & -0.2 & 2.3 & 2.7 & 2.8 & 2.4 & -- \\
\hline & 30 & -- & -- & -- & 3.7 & -- & -- & -- \\
\hline 63 & 15 & 113.2 & 1.9 & 5.5 & 5.8 & 5.8 & 5.4 & -- \\
\hline \multirow[t]{4}{*}{64} & 3 & -- & -0.1 & 1.5 & 3.0 & 2.5 & 2.7 & -- \\
\hline & 15 & 119.0 & -0.7 & 0.7 & 2.7 & 1.8 & 1.7 & -- \\
\hline & 30 & -- & -- & -- & 3.1 & -- & -- & -- \\
\hline & 60 & -- & -- & -- & 3.1 & -- & -- & -- \\
\hline \multirow[t]{3}{*}{55} & 3 & -- & 1.0 & 3.8 & & 4.8 & 4.5 & -- \\
\hline & 15 & 120.7 & 1.4 & 3.0 & 3.6 & 4.1 & 4.0 & -- \\
\hline & $30^{1}$ & -- & 2.7 & 3.8 & 5.9 & 5.8 & 5.4 & -- \\
\hline \multirow[t]{2}{*}{28} & 15 & 115.0 & -0.6 & 0.5 & 1.5 & 1.4 & 1.5 & -- \\
\hline & 30 & -- & -0.1 & 0.5 & 1.8 & 1.4 & 1.4 & -- \\
\hline \multirow[t]{2}{*}{62} & 15 & 115.7 & 0.3 & 0.8 & 5.3 & 5.5 & 5.6 & -- \\
\hline & 30 & -- & 0.9 & 1.2 & 5.6 & 5.8 & 6.0 & -- \\
\hline \multicolumn{9}{|c|}{ The Dalles downstream } \\
\hline \multirow[t]{2}{*}{29} & 15 & 117.8 & -0.2 & 0.0 & 0.0 & 0.1 & 0.3 & -- \\
\hline & 30 & -- & 0.2 & 0.2 & 0.4 & 0.5 & 0.5 & -- \\
\hline \multirow[t]{2}{*}{63} & 15 & 123.1 & 0.3 & 0.0 & 0.5 & 0.3 & 0.1 & -- \\
\hline & 30 & -- & 0.7 & 0.2 & 0.5 & 0.4 & 0.2 & -- \\
\hline \multicolumn{9}{|c|}{ Warrendale } \\
\hline \multirow[t]{2}{*}{45} & 15 & 127.4 & -0.1 & -0.3 & -3.4 & -3.2 & -3.1 & -- \\
\hline & 30 & -- & 0.2 & 0.0 & -3.0 & -2.8 & -- & -- \\
\hline \multicolumn{9}{|c|}{ Skamania } \\
\hline 45 & 15 & -- & 3.3 & 3.0 & 0.7 & -- & 0.2 & 123.9 \\
\hline \multicolumn{9}{|c|}{ Camas } \\
\hline \multirow[t]{2}{*}{35} & 15 & -- & -- & 2.5 & 3.2 & 3.2 & 2.7 & 124.3 \\
\hline & 30 & -- & -- & 2.6 & 3.3 & -- & -- & -- \\
\hline \multicolumn{9}{|c|}{ Kalama } \\
\hline 44 & 15 & -- & -4.7 & -2.0 & 0.9 & 0.6 & 0.1 & 116.9 \\
\hline \multicolumn{9}{|c|}{ Wauna Mill } \\
\hline 60 & 15 & 115.4 & 0.9 & 1.9 & 2.0 & 2.4 & 2.4 & -- \\
\hline
\end{tabular}

${ }^{1}$ Water temperature at this depth was 2 degrees Celsius higher than at the other depths. 
4. The probe's protective housing at each of the fixed stations could be modified to allow for improved water circulation and to maintain all probes at depths of about $15 \mathrm{ft}$.

5. A HYDROLAB unit could be operated concurrently with Common Sensing's instrument at selected stations for short-time periods (for example, a 2-week period) to further examine environmental variability and instrument variability.

6. In addition to spare miscellaneous monitoring supplies, it would be desirable to have available two DCPs and two TDG meters with probes and cables to be able to immediately replace nonworking equipment during the monitoring period.

7. It also would be desirable if all TDG and DCP equipment were available 1 month in advance of installation for verification that the equipment are functioning properly.

\section{REFERENCES CITED}

Jones, J. C., Tracey, D. C., and Sorensen, F. W., eds., 1991, Operating manual for the U.S. Geological Survey's data-collection system with the Geostationary Operational Environmental Satellite: U.S. Geological Survey Open-File Report 91-99, 237 p.

U.S. Environmental Protection Agency, 1986, Quality criteria for water: Washington, D.C., EPA-440-5-86-001. 


\section{SUPPLEMENTAL DATA}



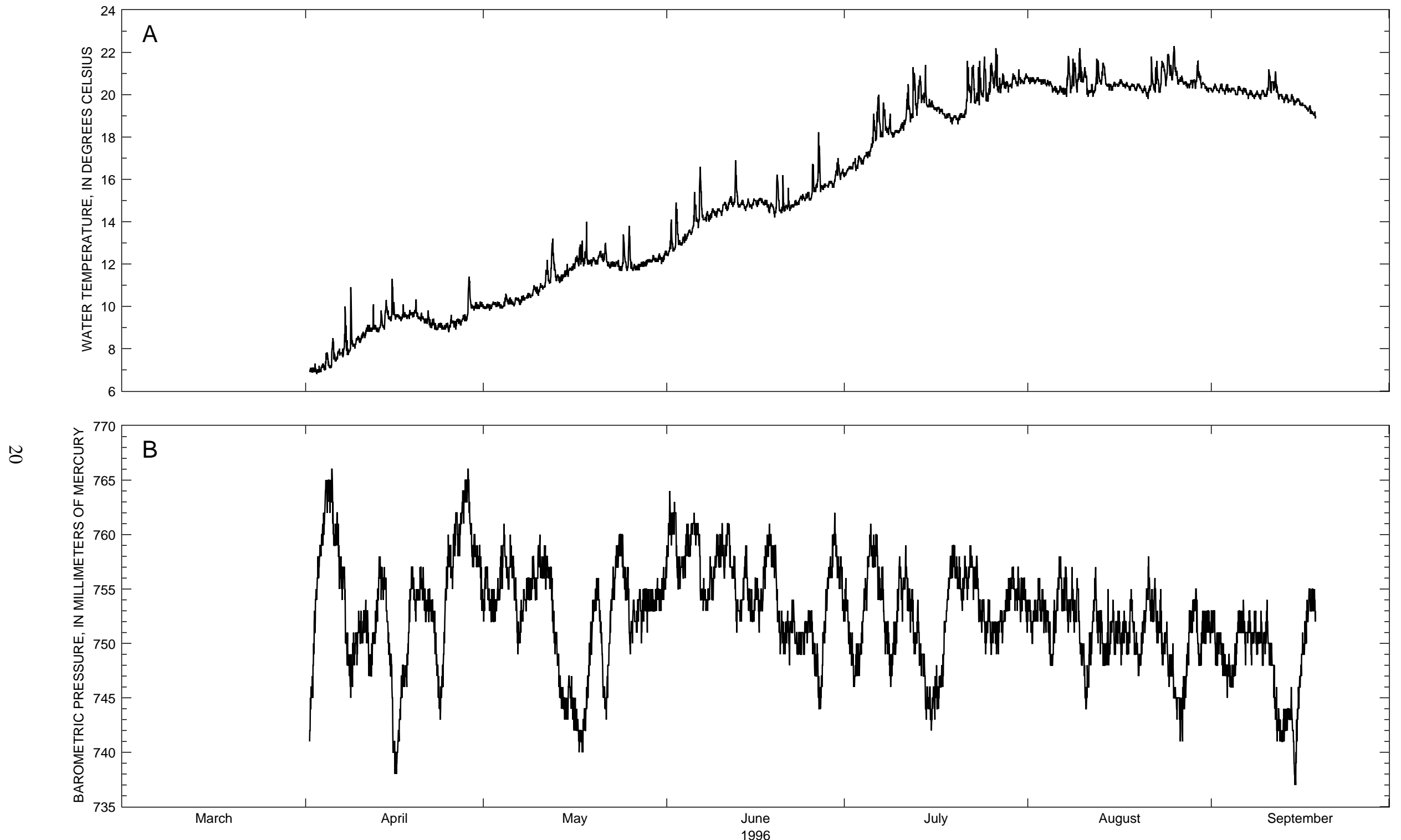

Figure 5. Hourly values of $(A)$ water temperature, $(B)$ barometric pressure, and (C, D) total dissolved gas at the Columbia River at John Day Dam forebay, Washington, April - September 1996. 

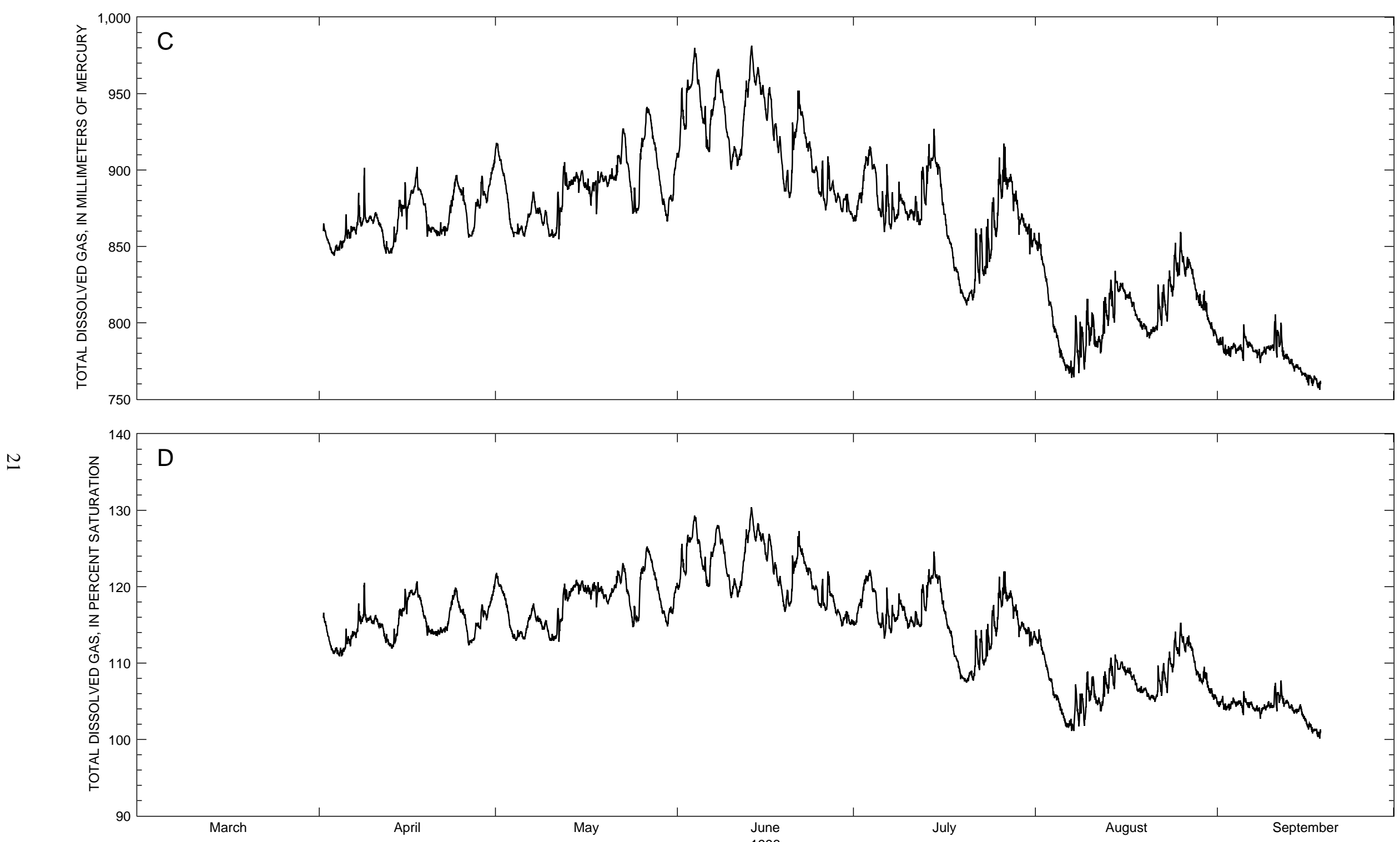

Figure 5. Hourly values of (A) water temperature, (B) barometric pressure, and (C, D) total dissolved gas at the Columbia River at John Day Dam forebay, Washington, April - September 1996.- continued 
Table 5. Daily summary of water temperature for the Columbia River at John Day Dam forebay, Washington, April - September 1996

[Values reported in degrees Celsius; Max, maximum; Min, minimum; Month, monthly summary statistics; --, not available; e, estimated]

\begin{tabular}{|c|c|c|c|c|c|c|c|c|c|c|c|c|}
\hline \multirow{2}{*}{ Day } & \multicolumn{3}{|c|}{ April } & \multicolumn{3}{|c|}{ May } & \multicolumn{3}{|c|}{ June } & \multicolumn{3}{|c|}{ July } \\
\hline & Max & Min & Mean & Max & Min & Mean & Max & Min & Mean & Max & Min & Mean \\
\hline 1 & -- & -- & -- & 10.1 & 9.9 & 10.0 & $\mathrm{e} 14.1$ & 12.4 & e12.9 & 16.6 & 16.2 & 16.4 \\
\hline 2 & 7.3 & 6.8 & 7.0 & 10.2 & 9.8 & 10.0 & $\mathrm{e} 14.9$ & 12.6 & e13.3 & 17.0 & 16.4 & 16.6 \\
\hline 3 & 7.3 & 6.9 & 7.1 & 10.2 & 9.9 & 10.1 & 13.3 & 12.9 & 13.0 & 17.1 & 16.5 & 16.8 \\
\hline 4 & 7.8 & 7.0 & 7.4 & 10.6 & 9.9 & 10.2 & 13.6 & 13.0 & 13.4 & 17.3 & 16.7 & 17.0 \\
\hline 5 & e8.5 & 7.1 & e7.6 & 10.4 & 10.1 & 10.2 & $\mathrm{e} 15.4$ & 13.4 & e14.0 & 19.1 & 17.1 & 17.6 \\
\hline 6 & 8.0 & 7.4 & 7.7 & 10.4 & 10.0 & 10.3 & e16.6 & 13.7 & e14.7 & e20.0 & 17.8 & e18.8 \\
\hline 7 & e10.0 & 7.6 & e8.2 & 10.5 & 10.1 & 10.3 & 14.5 & 14.0 & 14.2 & e19.6 & 18.0 & e 18.6 \\
\hline 8 & e10.9 & 7.7 & 8.3 & 10.6 & 10.3 & 10.5 & 14.6 & 14.0 & 14.3 & e19.1 & 18.1 & e18.4 \\
\hline 9 & 8.6 & 8.0 & 8.3 & 11.0 & 10.4 & 10.7 & 14.6 & 14.2 & 14.4 & 18.3 & 18.0 & 18.2 \\
\hline 10 & 8.8 & 8.3 & 8.5 & 11.1 & 10.5 & 10.8 & 14.9 & 14.3 & 14.7 & 18.7 & 18.2 & 18.4 \\
\hline 11 & 9.1 & 8.5 & 8.9 & $\mathrm{e} 12.2$ & 10.8 & e11.3 & 15.2 & 14.5 & 14.9 & e20.5 & 18.3 & e19.2 \\
\hline 12 & $\mathrm{e} 10.1$ & 8.8 & e9.1 & $\mathrm{e} 13.2$ & 11.1 & e11.9 & e16.9 & 14.7 & e15.3 & $\mathrm{e} 21.3$ & 18.7 & e19.7 \\
\hline 13 & e9.8 & 8.8 & e9.1 & $\mathrm{e} 12.0$ & 11.1 & 11.4 & 15.0 & 14.7 & 14.8 & e20.9 & 19.0 & $\mathrm{e} 20.0$ \\
\hline 14 & 10.3 & 8.9 & 9.5 & 11.7 & 11.2 & 11.4 & 15.1 & 14.5 & 14.8 & e21.4 & 19.5 & e19.9 \\
\hline 15 & $\mathrm{e} 11.3$ & 9.3 & e9.8 & 12.0 & 11.4 & 11.7 & 15.1 & 14.6 & 14.9 & 19.8 & 19.4 & 19.5 \\
\hline 16 & 9.6 & 9.4 & 9.5 & 12.4 & 11.7 & 12.0 & 15.1 & 14.7 & 15.0 & 19.5 & 19.2 & 19.4 \\
\hline 17 & 10.1 & 9.3 & 9.5 & e13.1 & 11.9 & $\mathrm{e} 12.4$ & 15.1 & 14.7 & 14.9 & 19.4 & 18.9 & 19.2 \\
\hline 18 & 9.8 & 9.4 & 9.6 & e 14.0 & 12.0 & e12.3 & 14.9 & 14.4 & 14.7 & 19.1 & 18.7 & 19.0 \\
\hline 19 & 10.3 & 9.5 & e9.7 & 12.3 & 11.9 & 12.1 & e16.2 & 14.2 & e15.0 & 19.0 & 18.6 & 18.9 \\
\hline 20 & 9.7 & 9.3 & 9.5 & 12.6 & 12.0 & 12.3 & e16.2 & 14.4 & e14.7 & 19.1 & 18.6 & 18.9 \\
\hline 21 & 9.8 & 9.1 & 9.4 & e13.0 & 12.1 & e12.4 & e15.6 & 14.5 & e14.7 & e21.6 & 18.9 & e19.6 \\
\hline 22 & 9.4 & 8.9 & 9.2 & 12.1 & 11.8 & 12.0 & 15.0 & 14.7 & 14.8 & e21.4 & e19.6 & e20.5 \\
\hline 23 & 9.2 & 8.9 & 9.0 & 12.2 & 11.7 & 12.0 & 15.3 & 14.8 & 15.0 & e21.6 & e19.5 & $\mathrm{e} 20.2$ \\
\hline 24 & 9.2 & 8.9 & 9.0 & $\mathrm{e} 13.4$ & 11.7 & $\mathrm{e} 12.2$ & 15.4 & 15.0 & 15.2 & $\mathrm{e} 21.8$ & $\mathrm{e} 19.7$ & $\mathrm{e} 20.5$ \\
\hline 25 & 9.6 & 8.8 & 9.2 & e13.8 & 11.7 & $\mathrm{e} 12.3$ & e16.7 & 15.0 & e15.5 & $\mathrm{e} 21.5$ & $\mathrm{e} 19.7$ & $\mathrm{e} 20.4$ \\
\hline 26 & 9.4 & 8.9 & 9.2 & 12.1 & 11.7 & 11.8 & e18.2 & 15.4 & e16.2 & $\mathrm{e} 22.2$ & e20.1 & e20.9 \\
\hline 27 & 9.6 & 9.1 & 9.4 & 12.1 & 11.7 & 11.9 & 15.8 & 15.4 & 15.7 & e21.0 & e20.1 & e20.5 \\
\hline 28 & e11.4 & 9.3 & e10.1 & 12.2 & 11.9 & 12.1 & 15.9 & 15.6 & 15.8 & 20.6 & 20.1 & e20.4 \\
\hline 29 & 10.2 & 9.8 & 10.0 & 12.4 & 12.1 & 12.2 & e17.0 & 15.6 & e16.1 & 21.0 & 20.3 & 20.7 \\
\hline 30 & 10.2 & 9.9 & 10.0 & 12.5 & 12.1 & 12.2 & 16.7 & 16.0 & 16.3 & e21.2 & 20.4 & e20.7 \\
\hline 31 & -- & -- & -- & 12.6 & 12.0 & 12.4 & -- & -- & -- & 21.0 & 20.5 & 20.7 \\
\hline Month & -- & -- & -- & 14.0 & 9.8 & 11.5 & 18.2 & 12.4 & 14.8 & 22.2 & 16.2 & 19.2 \\
\hline
\end{tabular}

\begin{tabular}{|c|c|c|c|c|c|c|}
\hline \multirow{2}{*}{ Day } & \multicolumn{3}{|c|}{ August } & \multicolumn{3}{|c|}{ September } \\
\hline & Max & Min & Mean & Max & Min & Mean \\
\hline 1 & 20.8 & 20.5 & 20.7 & 20.5 & 20.1 & 20.3 \\
\hline 2 & 20.8 & 20.5 & 20.7 & 20.5 & 20.1 & 20.3 \\
\hline 3 & 20.7 & 20.5 & 20.6 & 20.5 & 20.0 & 20.2 \\
\hline 4 & 20.7 & 20.4 & 20.5 & 20.3 & 20.1 & 20.2 \\
\hline 5 & 20.4 & 20.1 & 20.3 & 20.4 & 20.0 & 20.2 \\
\hline 6 & 20.4 & 20.0 & 20.2 & 20.3 & 20.0 & 20.2 \\
\hline 7 & $\mathrm{e} 21.8$ & 19.9 & $\mathrm{e} 20.6$ & 20.2 & 19.8 & 20.0 \\
\hline 8 & $\mathrm{e} 21.7$ & 20.1 & $\mathrm{e} 20.8$ & 20.2 & 19.8 & 20.0 \\
\hline 9 & $\mathrm{e} 22.2$ & 20.1 & e20.9 & 20.2 & 19.8 & 20.0 \\
\hline 10 & $\mathrm{e} 21.3$ & 20.0 & $\mathrm{e} 20.8$ & $\mathrm{e} 21.2$ & 19.9 & e20.3 \\
\hline 11 & 20.5 & 19.9 & 20.2 & $\mathrm{e} 21.1$ & e20.1 & $\mathrm{e} 20.5$ \\
\hline 12 & $\mathrm{e} 21.7$ & 19.9 & $\mathrm{e} 20.7$ & 20.5 & 19.8 & 20.0 \\
\hline 13 & $\mathrm{e} 21.5$ & $\mathrm{e} 20.5$ & e20.9 & 20.0 & 19.6 & 19.8 \\
\hline 14 & $\mathrm{e} 21.0$ & 20.2 & 20.5 & 20.0 & 19.5 & 19.8 \\
\hline 15 & 20.6 & 20.2 & 20.4 & 19.8 & 19.5 & 19.7 \\
\hline 16 & 20.7 & 20.4 & 20.5 & 19.6 & 19.4 & 19.5 \\
\hline 17 & 20.6 & 20.3 & 20.4 & 19.5 & 19.1 & 19.3 \\
\hline 18 & 20.5 & 20.2 & 20.4 & & & \\
\hline 19 & 20.5 & 20.2 & 20.4 & & & \\
\hline 20 & 20.4 & 20.1 & 20.2 & & & \\
\hline 21 & e21.8 & 19.8 & e20.4 & & & \\
\hline 22 & e21.6 & 20.1 & e20.7 & & & \\
\hline 23 & e21.6 & 20.1 & e20.8 & & & \\
\hline 24 & $\mathrm{e} 21.9$ & e20.4 & e21.2 & & & \\
\hline 25 & $\mathrm{e} 22.3$ & e20.7 & e21.3 & & & \\
\hline 26 & e21.3 & 20.5 & 20.8 & & & \\
\hline 27 & 20.9 & 20.3 & 20.6 & & & \\
\hline 28 & 20.7 & 20.3 & 20.5 & & & \\
\hline 29 & 21.6 & 20.3 & 20.8 & & & \\
\hline 30 & 21.0 & 20.3 & 20.5 & & & \\
\hline 31 & 20.4 & 20.1 & 20.3 & & & \\
\hline Month & 22.3 & 19.8 & 20.6 & & & \\
\hline
\end{tabular}


Table 6. Daily summary of barometric pressure for the Columbia River at John Day Dam forebay, Washington, April - September 1996

[Values reported in millimeters of mercury; Max, maximum; Min, minimum; Month, monthly summary statistics; --, not available]

\begin{tabular}{|c|c|c|c|c|c|c|c|c|c|c|c|c|}
\hline \multirow{2}{*}{ Day } & \multicolumn{3}{|c|}{ April } & \multicolumn{3}{|c|}{ May } & \multicolumn{3}{|c|}{ June } & \multicolumn{3}{|c|}{ July } \\
\hline & Max & Min & Mean & Max & Min & Mean & Max & Min & Mean & Max & Min & Mean \\
\hline 1 & -- & -- & -- & 757 & 752 & 755 & 764 & 757 & 760 & 756 & 749 & 752 \\
\hline 2 & 756 & 745 & 751 & 755 & 752 & 754 & 763 & 755 & 760 & 751 & 746 & 748 \\
\hline 3 & 761 & 756 & 758 & 758 & 752 & 755 & 758 & 755 & 757 & 752 & 747 & 749 \\
\hline 4 & 765 & 760 & 763 & 761 & 755 & 758 & 761 & 756 & 759 & 758 & 750 & 754 \\
\hline 5 & 766 & 759 & 762 & 760 & 755 & 757 & 762 & 759 & 761 & 761 & 757 & 758 \\
\hline 6 & 762 & 755 & 759 & 758 & 749 & 754 & 761 & 754 & 758 & 760 & 754 & 758 \\
\hline 7 & 757 & 748 & 754 & 755 & 750 & 752 & 756 & 753 & 754 & 757 & 749 & 754 \\
\hline 8 & 751 & 745 & 748 & 756 & 753 & 755 & 758 & 754 & 755 & 752 & 746 & 749 \\
\hline 9 & 753 & 748 & 750 & 758 & 754 & 756 & 760 & 756 & 758 & 755 & 747 & 750 \\
\hline 10 & 753 & 750 & 751 & 760 & 754 & 757 & 761 & 756 & 759 & 758 & 752 & 755 \\
\hline 11 & 754 & 747 & 750 & 759 & 755 & 757 & 761 & 757 & 759 & 759 & 753 & 756 \\
\hline 12 & 754 & 747 & 750 & 757 & 750 & 755 & 758 & 751 & 755 & 755 & 749 & 752 \\
\hline 13 & 758 & 752 & 755 & 751 & 745 & 749 & 756 & 752 & 753 & 754 & 747 & 750 \\
\hline 14 & 757 & 750 & 754 & 747 & 743 & 745 & 759 & 753 & 756 & 749 & 743 & 747 \\
\hline 15 & 751 & 740 & 746 & 747 & 743 & 745 & 756 & 752 & 753 & 746 & 742 & 744 \\
\hline 16 & 744 & 738 & 741 & 745 & 742 & 743 & 756 & 751 & 754 & 748 & 743 & 745 \\
\hline 17 & 748 & 743 & 747 & 743 & 740 & 742 & 760 & 753 & 756 & 752 & 745 & 748 \\
\hline 18 & 757 & 746 & 752 & 750 & 742 & 745 & 761 & 757 & 759 & 758 & 751 & 754 \\
\hline 19 & 757 & 752 & 755 & 755 & 748 & 752 & 759 & 751 & 755 & 759 & 756 & 757 \\
\hline 20 & 757 & 754 & 755 & 756 & 750 & 753 & 753 & 749 & 751 & 758 & 753 & 755 \\
\hline 21 & 755 & 752 & 754 & 751 & 743 & 746 & 753 & 748 & 751 & 758 & 754 & 756 \\
\hline 22 & 754 & 749 & 752 & 757 & 745 & 752 & 754 & 749 & 751 & 759 & 754 & 757 \\
\hline 23 & 751 & 743 & 746 & 760 & 754 & 757 & 751 & 749 & 750 & 758 & 752 & 755 \\
\hline 24 & 758 & 746 & 752 & 760 & 755 & 758 & 753 & 749 & 751 & 754 & 749 & 752 \\
\hline 25 & 760 & 755 & 758 & 757 & 749 & 753 & 753 & 747 & 751 & 754 & 749 & 752 \\
\hline 26 & 762 & 758 & 760 & 754 & 750 & 752 & 750 & 744 & 747 & 752 & 749 & 751 \\
\hline 27 & 765 & 758 & 762 & 755 & 750 & 753 & 755 & 744 & 750 & 753 & 749 & 752 \\
\hline 28 & 766 & 760 & 763 & 755 & 751 & 754 & 760 & 753 & 756 & 756 & 752 & 754 \\
\hline 29 & 761 & 757 & 758 & 755 & 753 & 753 & 762 & 756 & 759 & 757 & 753 & 755 \\
\hline 30 & 759 & 753 & 757 & 756 & 753 & 754 & 758 & 752 & 756 & 757 & 752 & 755 \\
\hline 31 & -- & -- & -- & 758 & 753 & 755 & -- & -- & -- & 756 & 751 & 754 \\
\hline Month & -- & -- & -- & 761 & 740 & 752 & 764 & 744 & 755 & 761 & 742 & 753 \\
\hline \multirow{2}{*}{ Day } & \multicolumn{3}{|c|}{ August } & \multicolumn{3}{|c|}{ September } & & & & & & \\
\hline & Max & Min & Mean & Max & Min & Mean & & & & & & \\
\hline 1 & 754 & 750 & 752 & 753 & 748 & 751 & & & & & & \\
\hline 2 & 756 & 752 & 753 & 751 & 747 & 750 & & & & & & \\
\hline 3 & 756 & 751 & 753 & 750 & 745 & 748 & & & & & & \\
\hline 4 & 754 & 748 & 751 & 750 & 746 & 747 & & & & & & \\
\hline 5 & 755 & 748 & 751 & 753 & 747 & 751 & & & & & & \\
\hline 6 & 758 & 753 & 755 & 754 & 748 & 751 & & & & & & \\
\hline 7 & 757 & 751 & 754 & 753 & 748 & 751 & & & & & & \\
\hline 8 & 757 & 751 & 753 & 753 & 749 & 751 & & & & & & \\
\hline 9 & 756 & 749 & 753 & 753 & 748 & 750 & & & & & & \\
\hline 10 & 750 & 744 & 747 & 754 & 748 & 751 & & & & & & \\
\hline 11 & 752 & 745 & 749 & 750 & 743 & 747 & & & & & & \\
\hline 12 & 757 & 750 & 753 & 746 & 741 & 742 & & & & & & \\
\hline 13 & 753 & 748 & 750 & 744 & 741 & 743 & & & & & & \\
\hline 14 & 755 & 748 & 750 & 746 & 739 & 743 & & & & & & \\
\hline 15 & 754 & 749 & 751 & 747 & 737 & 742 & & & & & & \\
\hline 16 & 753 & 748 & 751 & 752 & 746 & 749 & & & & & & \\
\hline 17 & 753 & 749 & 751 & 755 & 750 & 753 & & & & & & \\
\hline 18 & 755 & 749 & 752 & & & & & & & & & \\
\hline 19 & 751 & 747 & 749 & & & & & & & & & \\
\hline 20 & 753 & 748 & 751 & & & & & & & & & \\
\hline 21 & 758 & 752 & 755 & & & & & & & & & \\
\hline 22 & 756 & 750 & 753 & & & & & & & & & \\
\hline 23 & 755 & 748 & 751 & & & & & & & & & \\
\hline 24 & 752 & 746 & 749 & & & & & & & & & \\
\hline 25 & 750 & 745 & 747 & & & & & & & & & \\
\hline 26 & 745 & 741 & 744 & & & & & & & & & \\
\hline 27 & 750 & 741 & 746 & & & & & & & & & \\
\hline 28 & 754 & 748 & 752 & & & & & & & & & \\
\hline 29 & 755 & 748 & 752 & & & & & & & & & \\
\hline 30 & 753 & 748 & 750 & & & & & & & & & \\
\hline 31 & 753 & 750 & 752 & & & & & & & & & \\
\hline Month & 758 & 741 & 751 & & & & & & & & & \\
\hline
\end{tabular}


Table 7. Daily summary of total dissolved gas, in millimeters of mercury, for the Columbia River at John Day Dam forebay, Washington, April - September 1996

[Max, maximum; Min, minimum; Month, monthly summary statistics; --, not available; e, estimated]

\begin{tabular}{|c|c|c|c|c|c|c|c|c|c|c|c|c|}
\hline \multirow{2}{*}{ Day } & \multicolumn{3}{|c|}{ April } & \multicolumn{3}{|c|}{ May } & \multicolumn{3}{|c|}{ June } & \multicolumn{3}{|c|}{ July } \\
\hline & Max & Min & Mean & Max & Min & Mean & Max & Min & Mean & Max & Min & Mean \\
\hline 1 & -- & -- & -- & 918 & 904 & 912 & e953 & 908 & e926 & 883 & 866 & 873 \\
\hline 2 & 860 & 848 & 854 & 904 & 885 & 895 & e959 & 926 & e941 & 904 & 878 & 889 \\
\hline 3 & 851 & 844 & 847 & 885 & 859 & 869 & 980 & 953 & 962 & 915 & 903 & 909 \\
\hline 4 & 853 & 847 & 850 & 865 & 856 & 859 & 978 & 941 & 961 & 912 & 884 & 901 \\
\hline 5 & 871 & 853 & 858 & 864 & 856 & 860 & e942 & 915 & e933 & e886 & 869 & e876 \\
\hline 6 & 863 & 855 & 860 & 872 & 857 & 867 & 940 & 912 & 924 & e904 & 859 & e876 \\
\hline 7 & e885 & 858 & e866 & 886 & 871 & 878 & 966 & 936 & 953 & e885 & 861 & e870 \\
\hline 8 & e901 & 863 & e871 & 875 & 865 & 872 & 966 & 941 & 953 & e892 & 866 & e874 \\
\hline 9 & 870 & 866 & 868 & 874 & 861 & 867 & 942 & 909 & 925 & 884 & 872 & 878 \\
\hline 10 & 872 & 865 & 869 & 861 & 856 & 858 & 916 & 901 & 909 & 874 & 867 & 871 \\
\hline 11 & 867 & 853 & 862 & e886 & 855 & e866 & 916 & 903 & 909 & e883 & 866 & e873 \\
\hline 12 & e853 & 845 & 848 & e905 & 872 & e886 & 958 & 916 & 941 & e902 & 864 & e879 \\
\hline 13 & e863 & 846 & 850 & e898 & 887 & e981 & 981 & 947 & 968 & e917 & 877 & e893 \\
\hline 14 & e880 & 853 & e869 & 898 & 890 & 894 & 967 & 955 & 961 & e927 & 901 & e910 \\
\hline 15 & e892 & 861 & e876 & 900 & 885 & 895 & 959 & 939 & 951 & 905 & 888 & 900 \\
\hline 16 & 887 & 874 & 882 & 894 & 884 & 889 & 954 & 932 & 943 & 888 & 857 & 871 \\
\hline 17 & 902 & 886 & 892 & 892 & 877 & e887 & 946 & 919 & 928 & 857 & 842 & 852 \\
\hline 18 & 888 & 877 & 883 & e899 & 871 & 890 & 922 & 894 & 912 & 843 & 828 & 834 \\
\hline 19 & 878 & 856 & 865 & 898 & 892 & 895 & e900 & 885 & e891 & 828 & 815 & 820 \\
\hline 20 & 863 & 858 & 861 & 901 & 889 & 894 & e931 & 882 & e901 & 820 & 811 & 816 \\
\hline 21 & 866 & 857 & 859 & 909 & 893 & 898 & e952 & 921 & e935 & e862 & 815 & e828 \\
\hline 22 & 865 & 857 & 861 & 927 & 904 & 915 & 938 & 917 & 930 & e862 & 826 & e844 \\
\hline 23 & 888 & 864 & 877 & 924 & 889 & 906 & 918 & 899 & 913 & e868 & 831 & e842 \\
\hline 24 & 896 & 886 & 892 & 889 & 871 & e879 & 902 & 888 & 899 & e882 & 840 & e858 \\
\hline 25 & 889 & 874 & 882 & 921 & 872 & 892 & e906 & 880 & e888 & e908 & 856 & e874 \\
\hline 26 & 874 & 856 & 861 & 941 & 915 & 928 & e909 & 874 & e887 & e917 & 881 & e896 \\
\hline 27 & 881 & 857 & 869 & 940 & 918 & 931 & 893 & 880 & 887 & 897 & 887 & 893 \\
\hline 28 & e896 & 875 & e883 & 918 & 893 & 907 & 885 & 873 & 880 & 892 & 873 & 882 \\
\hline 29 & 890 & 878 & 884 & 893 & 873 & 882 & 884 & 872 & 879 & 874 & 858 & 868 \\
\hline 30 & 913 & 890 & 900 & 884 & 866 & 876 & 881 & 866 & 873 & 868 & 853 & 862 \\
\hline 31 & -- & -- & -- & 911 & 880 & 895 & -- & -- & -- & 862 & 845 & 854 \\
\hline Month & -- & -- & -- & 941 & 855 & 891 & 981 & 866 & 922 & 927 & 811 & 870 \\
\hline \multirow{2}{*}{ Day } & \multicolumn{3}{|c|}{ August } & \multicolumn{3}{|c|}{ September } & & & & & & \\
\hline & Max & Min & Mean & Max & Min & Mean & & & & & & \\
\hline 1 & 859 & 844 & 851 & 790 & 782 & 788 & & & & & & \\
\hline 2 & 844 & 825 & 835 & 786 & 779 & 782 & & & & & & \\
\hline 3 & 825 & 798 & 813 & 787 & 778 & 783 & & & & & & \\
\hline 4 & 799 & 784 & 793 & 786 & 780 & 783 & & & & & & \\
\hline 5 & 784 & 773 & 778 & 799 & 775 & 786 & & & & & & \\
\hline 6 & 773 & 767 & 770 & 788 & 783 & 785 & & & & & & \\
\hline 7 & e805 & 764 & e777 & 783 & 777 & 781 & & & & & & \\
\hline 8 & e801 & 767 & e785 & 784 & 774 & 779 & & & & & & \\
\hline 9 & e816 & 769 & e790 & 785 & 779 & 783 & & & & & & \\
\hline 10 & e806 & 785 & e797 & e805 & 782 & e789 & & & & & & \\
\hline 11 & e796 & 784 & e788 & e800 & 777 & e789 & & & & & & \\
\hline 12 & e817 & 780 & e789 & 787 & 776 & 779 & & & & & & \\
\hline 13 & e828 & 798 & $\mathrm{e} 812$ & 778 & 770 & 774 & & & & & & \\
\hline 14 & 834 & 800 & 817 & 773 & 768 & 771 & & & & & & \\
\hline 15 & 826 & 820 & 823 & 771 & 765 & 767 & & & & & & \\
\hline 16 & 822 & 815 & 818 & 766 & 759 & 764 & & & & & & \\
\hline 17 & 820 & 807 & 813 & 765 & 759 & 762 & & & & & & \\
\hline 18 & 807 & 798 & 803 & & & & & & & & & \\
\hline 19 & 801 & 794 & 797 & & & & & & & & & \\
\hline 20 & 797 & 790 & 793 & & & & & & & & & \\
\hline 21 & e825 & 794 & e800 & & & & & & & & & \\
\hline 22 & e825 & 798 & e812 & & & & & & & & & \\
\hline 23 & e834 & 801 & e816 & & & & & & & & & \\
\hline 24 & e852 & 817 & e831 & & & & & & & & & \\
\hline 25 & e859 & 830 & e841 & & & & & & & & & \\
\hline 26 & 844 & 831 & 838 & & & & & & & & & \\
\hline 27 & 842 & 829 & 835 & & & & & & & & & \\
\hline 28 & 829 & 811 & 819 & & & & & & & & & \\
\hline 29 & e821 & 808 & e813 & & & & & & & & & \\
\hline 30 & 814 & 796 & 803 & & & & & & & & & \\
\hline 31 & 800 & 787 & 794 & & & & & & & & & \\
\hline Month & 859 & 764 & 808 & & & & & & & & & \\
\hline
\end{tabular}


Table 8. Daily summary of total dissolved gas, in percent saturation, for the Columbia River at John Day Dam forebay, Washington, April - September 1996

[Max, maximum; Min, minimum; Month, monthly summary statistics; --, not available; e, estimated]

\begin{tabular}{|c|c|c|c|c|c|c|c|c|c|c|c|c|}
\hline \multirow{2}{*}{ Day } & \multicolumn{3}{|c|}{ April } & \multicolumn{3}{|c|}{ May } & \multicolumn{3}{|c|}{ June } & \multicolumn{3}{|c|}{ July } \\
\hline & Max & Min & Mean & Max & Min & Mean & Max & Min & Mean & Max & Min & Mean \\
\hline 1 & -- & -- & -- & 122 & 120 & 121 & e126 & 120 & e122 & 118 & 115 & 116 \\
\hline 2 & 115 & 112 & 114 & 120 & 117 & 119 & $\mathrm{e} 127$ & 122 & e124 & 121 & 117 & 119 \\
\hline 3 & 112 & 111 & 112 & 118 & 113 & 115 & 129 & 126 & 127 & 122 & 120 & 121 \\
\hline 4 & 112 & 111 & 111 & 114 & 113 & 113 & 129 & 124 & 127 & 121 & 117 & 119 \\
\hline 5 & 114 & 111 & 113 & 114 & 113 & 114 & e124 & 120 & e123 & e117 & 115 & e115 \\
\hline 6 & 114 & 112 & 113 & 116 & 113 & 115 & 124 & 120 & 122 & e120 & 113 & e116 \\
\hline 7 & e118 & 113 & e115 & 118 & 116 & 117 & 128 & 124 & 126 & e118 & 114 & e115 \\
\hline 8 & $\mathrm{e} 120$ & 115 & e117 & 116 & 114 & 116 & 128 & 124 & 126 & e119 & 115 & e117 \\
\hline 9 & 116 & 115 & 116 & 116 & 114 & 115 & 125 & 120 & 122 & 118 & 115 & 117 \\
\hline 10 & 116 & 115 & 116 & 114 & 113 & 113 & 121 & 118 & 120 & 116 & 114 & 115 \\
\hline 11 & 115 & 114 & 115 & e117 & 113 & e114 & 121 & 119 & 120 & e117 & 115 & e116 \\
\hline 12 & e114 & 112 & 113 & e120 & 115 & e117 & 127 & 121 & 125 & e120 & 115 & e117 \\
\hline 13 & $\mathrm{e} 114$ & 112 & 113 & e120 & 118 & e119 & 130 & 126 & 128 & e122 & 117 & 119 \\
\hline 14 & e117 & 113 & e115 & 121 & 119 & 120 & 128 & 126 & 127 & e125 & 120 & e122 \\
\hline 15 & $\mathrm{e} 120$ & 116 & e117 & 121 & 119 & 120 & 127 & 124 & 126 & 122 & 119 & 121 \\
\hline 16 & 120 & 118 & 119 & 120 & 119 & 120 & 127 & 123 & 125 & 119 & 115 & 117 \\
\hline 17 & 121 & 119 & 119 & 121 & 118 & e120 & 125 & 121 & 123 & 115 & 112 & 114 \\
\hline 18 & 119 & 116 & 117 & e121 & 117 & 119 & 121 & 118 & 120 & 112 & 109 & 111 \\
\hline 19 & 116 & 114 & 115 & 120 & 118 & 119 & e120 & 117 & e118 & 110 & 108 & 108 \\
\hline 20 & 114 & 114 & 114 & 120 & 118 & 119 & e124 & 117 & e120 & 109 & 107 & 108 \\
\hline 21 & 115 & 114 & 114 & 122 & 119 & 120 & e127 & 122 & e125 & e114 & 108 & e110 \\
\hline 22 & 115 & 114 & 115 & 123 & 120 & 122 & 125 & 122 & 124 & e114 & 109 & e111 \\
\hline 23 & 119 & 115 & 118 & 122 & 117 & 120 & 122 & 120 & 122 & e115 & 110 & e112 \\
\hline 24 & 120 & 117 & 119 & 117 & 115 & e116 & 120 & 118 & 120 & e118 & 112 & e114 \\
\hline 25 & 117 & 115 & 116 & 122 & 115 & 118 & e121 & 117 & e118 & e121 & 113 & e116 \\
\hline 26 & 115 & 112 & 113 & 125 & 122 & 123 & e122 & 117 & e119 & e122 & 117 & e119 \\
\hline 27 & 115 & 113 & 114 & 125 & 122 & 124 & 119 & 116 & 118 & 119 & 118 & 119 \\
\hline 28 & e118 & 114 & e116 & 122 & 118 & 120 & 117 & 115 & 116 & 118 & 116 & 117 \\
\hline 29 & 117 & 116 & 117 & 118 & 116 & 117 & 117 & 115 & 116 & 116 & 113 & 115 \\
\hline 30 & 121 & 117 & 119 & 117 & 115 & 116 & 116 & 115 & 116 & 115 & 113 & 114 \\
\hline 31 & -- & -- & -- & 120 & 116 & 118 & -- & -- & -- & 114 & 112 & 113 \\
\hline Month & -- & -- & -- & 125 & 113 & 118 & 130 & 115 & 122 & 125 & 107 & 116 \\
\hline \multirow{2}{*}{ Day } & \multicolumn{3}{|c|}{ August } & \multicolumn{3}{|c|}{ September } & & & & & & \\
\hline & Max & Min & Mean & Max & Min & Mean & & & & & & \\
\hline 1 & 114 & 112 & 113 & 106 & 104 & 105 & & & & & & \\
\hline 2 & 112 & 109 & 111 & 105 & 104 & 104 & & & & & & \\
\hline 3 & 109 & 106 & 108 & 105 & 104 & 105 & & & & & & \\
\hline 4 & 106 & 105 & 106 & 105 & 104 & 105 & & & & & & \\
\hline 5 & 105 & 102 & 104 & 106 & 103 & 105 & & & & & & \\
\hline 6 & 102 & 102 & 102 & 105 & 104 & 105 & & & & & & \\
\hline 7 & e107 & 101 & e103 & 104 & 104 & 104 & & & & & & \\
\hline 8 & e106 & 102 & e104 & 105 & 103 & 104 & & & & & & \\
\hline 9 & e109 & 102 & e105 & 105 & 104 & 104 & & & & & & \\
\hline 10 & e108 & 105 & e107 & e107 & 104 & e105 & & & & & & \\
\hline 11 & e106 & 104 & e105 & e108 & 104 & e106 & & & & & & \\
\hline 12 & e109 & 104 & e106 & 106 & 104 & 105 & & & & & & \\
\hline 13 & e111 & 106 & e108 & 105 & 103 & 104 & & & & & & \\
\hline 14 & 111 & 107 & 109 & 104 & 103 & 104 & & & & & & \\
\hline 15 & 110 & 109 & 110 & 105 & 102 & 103 & & & & & & \\
\hline 16 & 109 & 108 & 109 & 103 & 101 & 102 & & & & & & \\
\hline 17 & 109 & 108 & 108 & 102 & 101 & 101 & & & & & & \\
\hline 18 & 108 & 106 & 107 & & & & & & & & & \\
\hline 19 & 107 & 106 & 106 & & & & & & & & & \\
\hline 20 & 106 & 105 & 106 & & & & & & & & & \\
\hline 21 & e110 & 105 & e106 & & & & & & & & & \\
\hline 22 & $\mathrm{e} 110$ & 106 & e108 & & & & & & & & & \\
\hline 23 & e111 & 106 & e109 & & & & & & & & & \\
\hline 24 & e114 & 109 & e111 & & & & & & & & & \\
\hline 25 & e115 & 111 & e113 & & & & & & & & & \\
\hline 26 & 113 & 111 & 113 & & & & & & & & & \\
\hline 27 & 114 & 110 & 112 & & & & & & & & & \\
\hline 28 & 110 & 108 & 109 & & & & & & & & & \\
\hline 29 & e109 & 107 & e108 & & & & & & & & & \\
\hline 30 & 109 & 106 & 107 & & & & & & & & & \\
\hline 31 & 107 & 105 & 106 & & & & & & & & & \\
\hline Month & 115 & 101 & 108 & & & & & & & & & \\
\hline
\end{tabular}



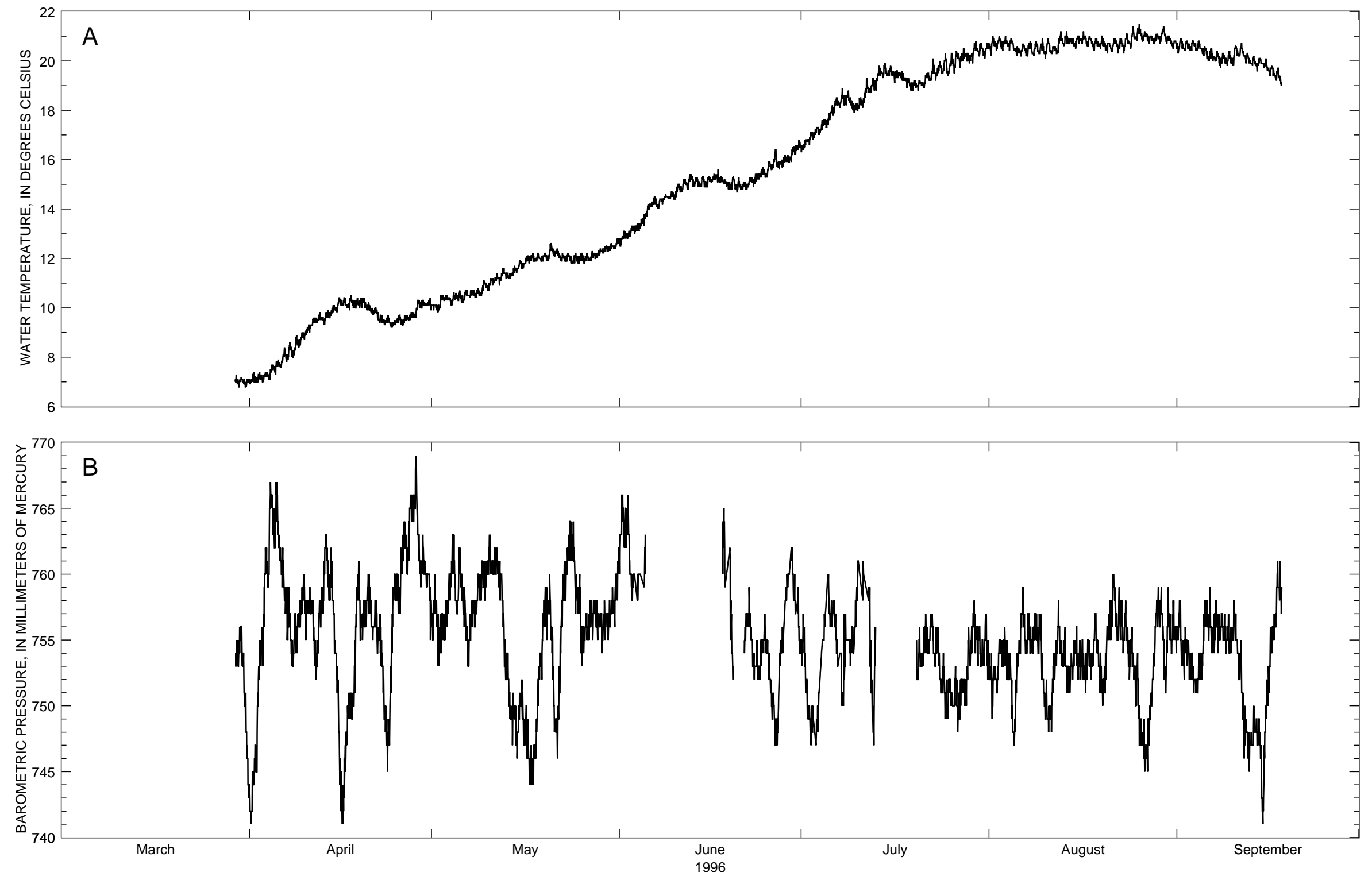

Figure 6. Hourly values of (A) water temperature, (B) barometric pressure, and (C, D) total dissolved gas at the Columbia River, right bank, near Cliffs, Washington, MarchSeptember 1996. 


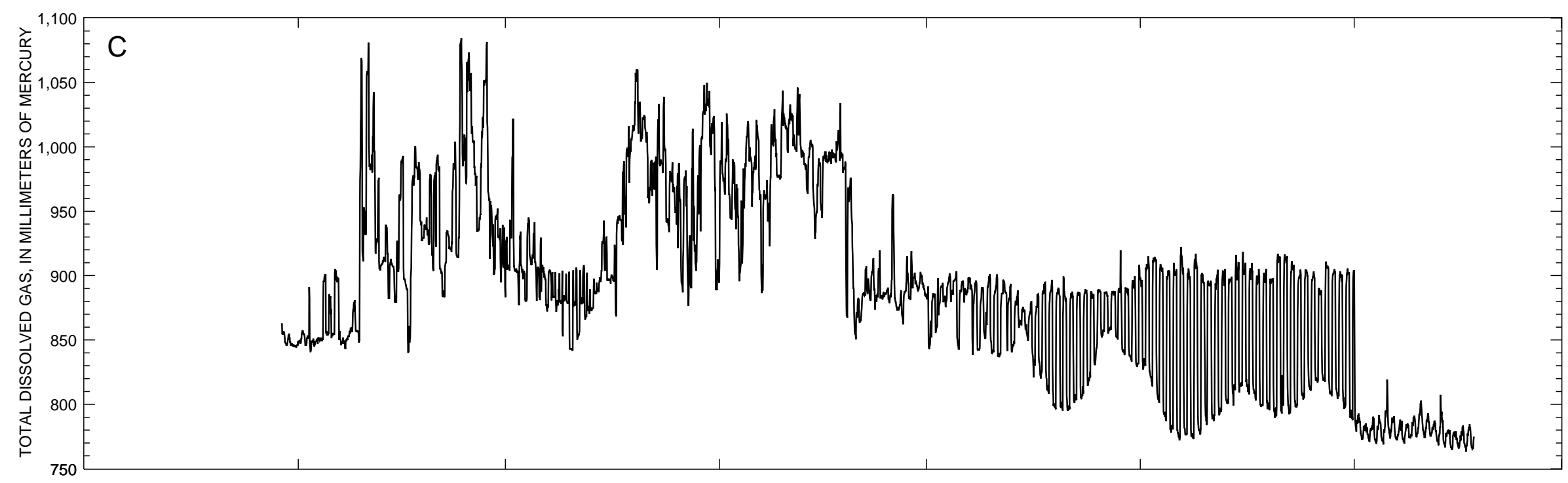

N

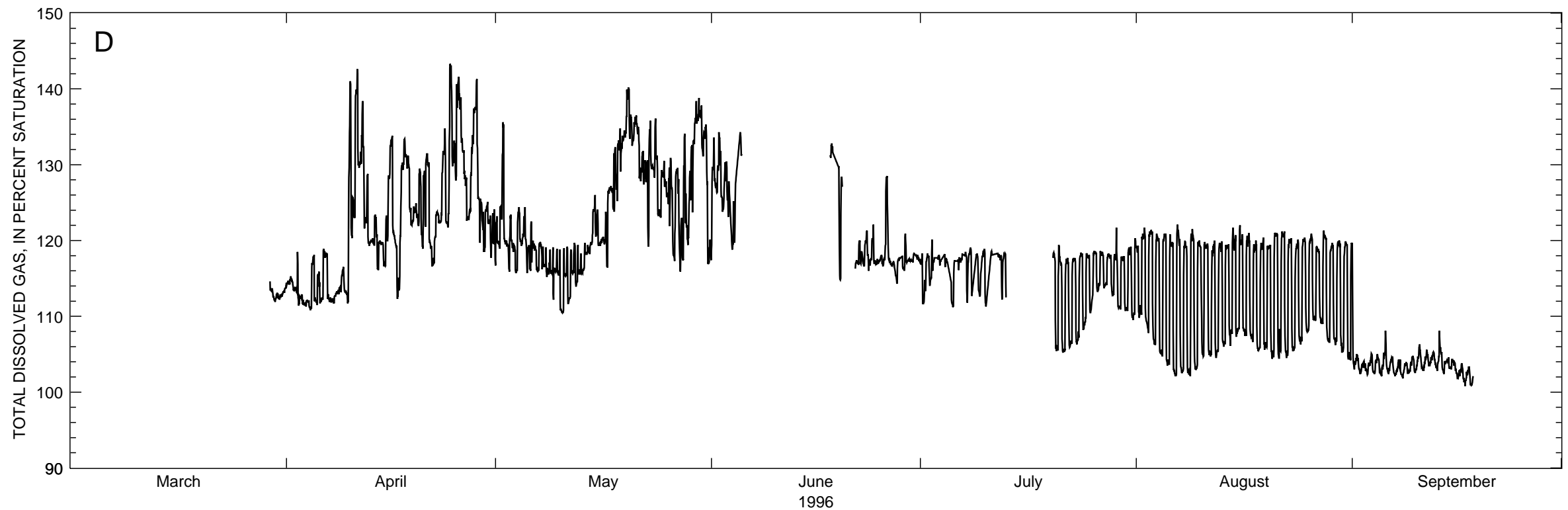

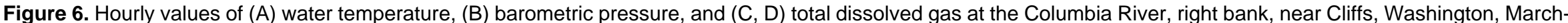
September 1996.-Continued 
Table 9. Daily summary of water temperature for the Columbia River, right bank, near Cliffs, Washington, March - September 1996

[Values report in degrees Celsius; Max, maximum; Min, minimum; Month, monthly summary statistics; --, not available]

\begin{tabular}{|c|c|c|c|c|c|c|c|c|c|c|c|c|}
\hline \multirow{2}{*}{ Day } & \multicolumn{3}{|c|}{ March } & \multicolumn{3}{|c|}{ April } & \multicolumn{3}{|c|}{ May } & \multicolumn{3}{|c|}{ June } \\
\hline & Max & Min & Mean & Max & Min & Mean & Max & Min & Mean & Max & Min & Mean \\
\hline 1 & & & & 7.4 & 6.9 & 7.1 & 10.1 & 9.9 & 10.1 & 13.1 & 12.5 & 12.8 \\
\hline 2 & & & & 7.4 & 7.0 & 7.2 & 10.5 & 9.8 & 10.2 & 13.2 & 12.8 & 13.0 \\
\hline 3 & & & & 7.4 & 7.0 & 7.2 & 10.5 & 10.3 & 10.4 & 13.4 & 13.0 & 13.2 \\
\hline 4 & & & & 7.7 & 7.1 & 7.4 & 10.6 & 10.1 & 10.4 & 13.6 & 13.1 & 13.4 \\
\hline 5 & & & & 7.9 & 7.3 & 7.7 & 10.7 & 10.2 & 10.4 & 14.2 & 13.3 & 13.8 \\
\hline 6 & & & & 8.4 & 7.6 & 7.9 & 10.7 & 10.2 & 10.5 & 14.5 & 14.0 & 14.2 \\
\hline 7 & & & & 8.6 & 7.8 & 8.2 & 10.7 & 10.4 & 10.6 & 14.4 & 14.0 & 14.3 \\
\hline 8 & & & & 8.9 & 8.0 & 8.4 & 10.8 & 10.4 & 10.6 & 14.6 & 14.2 & 14.4 \\
\hline 9 & & & & 9.0 & 8.4 & 8.7 & 11.1 & 10.5 & 10.8 & 14.7 & 14.4 & 14.5 \\
\hline 10 & & & & 9.3 & 8.7 & 9.1 & 11.2 & 10.7 & 10.9 & 15.0 & 14.4 & 14.7 \\
\hline 11 & & & & 9.6 & 9.2 & 9.4 & 11.4 & 10.9 & 11.1 & 15.2 & 14.7 & 15.0 \\
\hline 12 & & & & 9.8 & 9.4 & 9.6 & 11.6 & 10.9 & 11.3 & 15.4 & 14.8 & 15.1 \\
\hline 13 & & & & 9.9 & 9.3 & 9.6 & 11.4 & 11.2 & 11.3 & 15.3 & 14.9 & 15.1 \\
\hline 14 & & & & 10.1 & 9.6 & 9.8 & 11.7 & 11.2 & 11.4 & 15.3 & 14.9 & 15.1 \\
\hline 15 & & & & 10.4 & 9.8 & 10.1 & 11.9 & 11.4 & 11.7 & 15.3 & 14.9 & 15.1 \\
\hline 16 & & & & 10.4 & 10.1 & 10.2 & 12.1 & 11.6 & 11.9 & 15.4 & 15.1 & 15.2 \\
\hline 17 & & & & 10.5 & 9.9 & 10.2 & 12.2 & 11.8 & 12.0 & 15.6 & 15.1 & 15.2 \\
\hline 18 & & & & 10.4 & 10.0 & 10.2 & 12.2 & 11.9 & 12.0 & 15.3 & 14.8 & 15.1 \\
\hline 19 & & & & 10.4 & 10.0 & 10.2 & 12.2 & 11.9 & 12.0 & 15.3 & 14.8 & 15.0 \\
\hline 20 & & & & 10.2 & 9.9 & 10.0 & 12.6 & 11.9 & 12.2 & 15.3 & 14.7 & 14.9 \\
\hline 21 & & & & 10.0 & 9.7 & 9.9 & 12.4 & 12.1 & 12.2 & 15.1 & 14.8 & 14.9 \\
\hline 22 & & & & 9.9 & 9.4 & 9.6 & 12.2 & 11.9 & 12.0 & 15.3 & 14.8 & 15.1 \\
\hline 23 & & & & 9.7 & 9.3 & 9.5 & 12.2 & 11.9 & 12.0 & 15.4 & 14.9 & 15.2 \\
\hline 24 & & & & 9.5 & 9.2 & 9.4 & 12.2 & 11.8 & 12.0 & 15.7 & 15.2 & 15.5 \\
\hline 25 & & & & 9.7 & 9.3 & 9.5 & 12.2 & 11.8 & 12.0 & 15.9 & 15.3 & 15.6 \\
\hline 26 & & & & 9.7 & 9.3 & 9.5 & 12.2 & 11.8 & 12.0 & 16.4 & 15.6 & 15.9 \\
\hline 27 & & & & 9.8 & 9.5 & 9.6 & 12.3 & 11.9 & 12.1 & 16.0 & 15.6 & 15.8 \\
\hline 28 & & & & 10.3 & 9.6 & 9.9 & 12.4 & 12.0 & 12.2 & 16.2 & 15.7 & 16.0 \\
\hline 29 & & & & 10.3 & 10.0 & 10.2 & 12.5 & 12.2 & 12.4 & 16.5 & 15.9 & 16.2 \\
\hline 30 & 7.2 & 6.8 & 7.0 & 10.4 & 9.9 & 10.1 & 12.6 & 12.3 & 12.4 & 16.8 & 16.2 & 16.5 \\
\hline 31 & 7.1 & 6.8 & 7.0 & -- & -- & -- & 12.8 & 12.4 & 12.6 & -- & -- & -- \\
\hline Month & -- & -- & -- & 10.5 & 6.9 & 9.2 & 12.8 & 9.8 & 11.5 & 16.8 & 12.5 & 14.9 \\
\hline \multirow{2}{*}{ Day } & \multicolumn{3}{|c|}{ July } & \multicolumn{3}{|c|}{ August } & \multicolumn{3}{|c|}{ September } & & & \\
\hline & Max & Min & Mean & Max & Min & Mean & Max & Min & Mean & & & \\
\hline 1 & 16.8 & 16.3 & 16.6 & 21.0 & 20.2 & 20.6 & 20.9 & 20.3 & 20.6 & & & \\
\hline 2 & 17.1 & 16.6 & 16.9 & 21.0 & 20.4 & 20.7 & 20.8 & 20.4 & 20.6 & & & \\
\hline 3 & 17.3 & 16.9 & 17.1 & 21.0 & 20.4 & 20.7 & 20.8 & 20.4 & 20.6 & & & \\
\hline 4 & 17.6 & 17.1 & 17.4 & 20.9 & 20.4 & 20.7 & 20.8 & 20.4 & 20.6 & & & \\
\hline 5 & 18.0 & 17.3 & 17.6 & 20.6 & 20.2 & 20.5 & 20.6 & 20.1 & 20.4 & & & \\
\hline 6 & 18.5 & 17.8 & 18.2 & 20.7 & 20.2 & 20.5 & 20.5 & 20.0 & 20.2 & & & \\
\hline 7 & 18.9 & 18.1 & 18.4 & 20.8 & 20.2 & 20.5 & 20.4 & 19.9 & 20.1 & & & \\
\hline 8 & 18.8 & 18.2 & 18.5 & 20.8 & 20.2 & 20.5 & 20.3 & 19.7 & 20.1 & & & \\
\hline 9 & 18.5 & 17.9 & 18.2 & 20.9 & 20.3 & 20.5 & 20.4 & 19.8 & 20.1 & & & \\
\hline 10 & 18.5 & 18.0 & 18.2 & 20.9 & 20.1 & 20.5 & 20.6 & 19.8 & 20.3 & & & \\
\hline 11 & 19.3 & 18.1 & 18.6 & 20.6 & 20.3 & 20.5 & 20.7 & 20.2 & 20.4 & & & \\
\hline 12 & 19.3 & 18.7 & 18.9 & 21.2 & 20.3 & 20.7 & 20.3 & 19.9 & 20.1 & & & \\
\hline 13 & 19.8 & 18.8 & 19.3 & 21.2 & 20.6 & 20.9 & 20.2 & 19.8 & 20.0 & & & \\
\hline 14 & 19.9 & 19.3 & 19.6 & 20.9 & 20.6 & 20.8 & 20.1 & 19.6 & 19.9 & & & \\
\hline 15 & 19.8 & 19.4 & 19.5 & 21.0 & 20.5 & 20.8 & 20.1 & 19.6 & 19.8 & & & \\
\hline 16 & 19.6 & 19.1 & 19.5 & 21.2 & 20.7 & 20.9 & 19.8 & 19.4 & 19.6 & & & \\
\hline 17 & 19.6 & 19.2 & 19.4 & 21.0 & 20.6 & 20.8 & 19.7 & 19.2 & 19.4 & & & \\
\hline 18 & 19.3 & 18.9 & 19.2 & 20.9 & 20.4 & 20.7 & & & & & & \\
\hline 19 & 19.2 & 18.8 & 19.1 & 21.0 & 20.5 & 20.7 & & & & & & \\
\hline 20 & 19.2 & 18.8 & 19.1 & 20.7 & 20.3 & 20.6 & & & & & & \\
\hline 21 & 19.5 & 18.9 & 19.2 & 20.9 & 20.4 & 20.6 & & & & & & \\
\hline 22 & 20.1 & 19.2 & 19.5 & 20.9 & 20.3 & 20.7 & & & & & & \\
\hline 23 & 20.0 & 19.2 & 19.6 & 21.1 & 20.3 & 20.8 & & & & & & \\
\hline 24 & 20.3 & 19.5 & 19.8 & 21.4 & 20.5 & 20.9 & & & & & & \\
\hline 25 & 20.3 & 19.4 & 19.8 & 21.5 & 20.8 & 21.1 & & & & & & \\
\hline 26 & 20.4 & 19.5 & 20.1 & 21.2 & 20.8 & 21.0 & & & & & & \\
\hline 27 & 20.5 & 19.7 & 20.1 & 21.1 & 20.7 & 20.9 & & & & & & \\
\hline 28 & 20.3 & 19.9 & 20.2 & 21.1 & 20.7 & 20.9 & & & & & & \\
\hline 29 & 20.8 & 19.9 & 20.4 & 21.4 & 20.7 & 21.1 & & & & & & \\
\hline 30 & 20.7 & 20.2 & 20.5 & 21.1 & 20.7 & 20.9 & & & & & & \\
\hline 31 & 20.7 & 20.1 & 20.5 & 20.8 & 20.5 & 20.7 & & & & & & \\
\hline Month & 20.8 & 16.3 & 19.0 & 21.5 & 20.1 & 20.7 & & & & & & \\
\hline
\end{tabular}


Table 10. Daily summary of barometric pressure for the Columbia River, right bank, near Cliffs, Washington, March - September 1996

[Values reported in millimeters of mercury; Max, maximum; Min, minimum; Month, monthly summary statistics; --, not available]

\begin{tabular}{|c|c|c|c|c|c|c|c|c|c|c|c|c|}
\hline \multirow{2}{*}{ Day } & \multicolumn{3}{|c|}{ March } & \multicolumn{3}{|c|}{ April } & \multicolumn{3}{|c|}{ May } & \multicolumn{3}{|c|}{ June } \\
\hline & Max & Min & Mean & Max & Min & Mean & Max & Min & Mean & Max & Min & Mean \\
\hline 1 & & & & 747 & 741 & 744 & 760 & 755 & 757 & 766 & 760 & 764 \\
\hline 2 & & & & 755 & 745 & 751 & 757 & 753 & 755 & -- & -- & -- \\
\hline 3 & & & & 762 & 753 & 758 & 760 & 755 & 757 & -- & -- & -- \\
\hline 4 & & & & 767 & 759 & 764 & 763 & 758 & 760 & -- & -- & -- \\
\hline 5 & & & & 767 & 762 & 764 & 762 & 757 & 759 & -- & -- & -- \\
\hline 6 & & & & 762 & 757 & 760 & 760 & 753 & 757 & -- & -- & -- \\
\hline 7 & & & & 765 & 758 & 761 & 758 & 753 & 756 & -- & -- & -- \\
\hline 8 & & & & 757 & 753 & 755 & 760 & 756 & 758 & -- & -- & -- \\
\hline 9 & & & & 760 & 756 & 758 & 761 & 758 & 760 & -- & -- & -- \\
\hline 10 & & & & 759 & 755 & 758 & 763 & 759 & 761 & -- & -- & -- \\
\hline 11 & & & & 758 & 752 & 756 & 762 & 760 & 761 & -- & -- & -- \\
\hline 12 & & & & 759 & 752 & 756 & 761 & 755 & 759 & -- & -- & -- \\
\hline 13 & & & & 763 & 757 & 761 & 755 & 749 & 752 & -- & -- & -- \\
\hline 14 & & & & 762 & 754 & 759 & 751 & 747 & 749 & -- & -- & -- \\
\hline 15 & & & & 755 & 744 & 751 & 752 & 746 & 749 & -- & -- & -- \\
\hline 16 & & & & 748 & 741 & 744 & 751 & 746 & 748 & -- & -- & -- \\
\hline 17 & & & & 751 & 747 & 750 & 747 & 744 & 745 & -- & -- & -- \\
\hline 18 & & & & 761 & 750 & 755 & 754 & 746 & 750 & -- & -- & -- \\
\hline 19 & & & & 761 & 755 & 757 & 759 & 752 & 756 & -- & -- & -- \\
\hline 20 & & & & 760 & 756 & 758 & 760 & 754 & 757 & -- & -- & -- \\
\hline 21 & & & & 758 & 755 & 756 & 755 & 746 & 749 & -- & -- & -- \\
\hline 22 & & & & 757 & 753 & 755 & 761 & 749 & 756 & 759 & 753 & 756 \\
\hline 23 & & & & 753 & 745 & 749 & 764 & 758 & 761 & 755 & 752 & 753 \\
\hline 24 & & & & 760 & 747 & 755 & 764 & 757 & 761 & 757 & 752 & 754 \\
\hline 25 & & & & 762 & 757 & 759 & 760 & 753 & 757 & 757 & 751 & 754 \\
\hline 26 & & & & 764 & 760 & 762 & 757 & 754 & 756 & -- & -- & -- \\
\hline 27 & & & & 766 & 760 & 764 & 758 & 755 & 757 & -- & -- & -- \\
\hline 28 & & & & 769 & 761 & 765 & 758 & 755 & 757 & -- & -- & -- \\
\hline 29 & & & & 763 & 759 & 761 & 758 & 754 & 756 & -- & -- & -- \\
\hline 30 & 756 & 753 & 754 & 760 & 755 & 759 & 759 & 755 & 757 & -- & -- & -- \\
\hline 31 & 754 & 743 & 748 & -- & -- & -- & 762 & 756 & 759 & -- & -- & -- \\
\hline Month & -- & -- & -- & 769 & 741 & 757 & 764 & 744 & 756 & -- & -- & -- \\
\hline \multirow{2}{*}{ Day } & \multicolumn{3}{|c|}{ July } & \multicolumn{3}{|c|}{ August } & \multicolumn{3}{|c|}{ September } & & & \\
\hline & Max & Min & Mean & Max & Min & Mean & Max & Min & Mean & & & \\
\hline 1 & -- & -- & -- & 754 & 749 & 752 & 758 & 753 & 755 & & & \\
\hline 2 & -- & -- & -- & 755 & 751 & 753 & 756 & 752 & 754 & & & \\
\hline 3 & -- & -- & -- & 756 & 752 & 754 & 754 & 751 & 753 & & & \\
\hline 4 & -- & -- & -- & 754 & 748 & 752 & 754 & 751 & 752 & & & \\
\hline 5 & -- & -- & -- & 755 & 747 & 751 & 758 & 752 & 756 & & & \\
\hline 6 & -- & -- & -- & 759 & 754 & 756 & 759 & 754 & 756 & & & \\
\hline 7 & -- & -- & -- & 757 & 754 & 755 & 758 & 754 & 756 & & & \\
\hline 8 & -- & -- & -- & 757 & 754 & 756 & 757 & 755 & 756 & & & \\
\hline 9 & -- & -- & -- & 757 & 751 & 754 & 757 & 754 & 755 & & & \\
\hline 10 & -- & -- & -- & 752 & 748 & 750 & 758 & 754 & 756 & & & \\
\hline 11 & -- & -- & -- & 755 & 748 & 751 & 755 & 749 & 752 & & & \\
\hline 12 & -- & -- & -- & 758 & 753 & 755 & 750 & 745 & 748 & & & \\
\hline 13 & -- & -- & -- & 756 & 751 & 753 & 750 & 747 & 748 & & & \\
\hline 14 & -- & -- & -- & 755 & 751 & 753 & 749 & 743 & 747 & & & \\
\hline 15 & -- & -- & -- & 756 & 752 & 754 & 752 & 741 & 747 & & & \\
\hline 16 & -- & -- & -- & 755 & 751 & 753 & 756 & 750 & 754 & & & \\
\hline 17 & -- & -- & -- & 755 & 752 & 754 & 761 & 755 & 758 & & & \\
\hline 18 & -- & -- & -- & 757 & 753 & 755 & & & & & & \\
\hline 19 & -- & -- & -- & 754 & 750 & 752 & & & & & & \\
\hline 20 & -- & -- & -- & 758 & 751 & 755 & & & & & & \\
\hline 21 & 757 & 753 & 755 & 760 & 755 & 758 & & & & & & \\
\hline 22 & 757 & 754 & 755 & 759 & 755 & 757 & & & & & & \\
\hline 23 & 756 & 752 & 754 & 758 & 753 & 755 & & & & & & \\
\hline 24 & 754 & 749 & 751 & 756 & 752 & 754 & & & & & & \\
\hline 25 & 752 & 749 & 751 & 754 & 747 & 751 & & & & & & \\
\hline 26 & 753 & 748 & 750 & 749 & 745 & 747 & & & & & & \\
\hline 27 & 752 & 749 & 751 & 752 & 745 & 749 & & & & & & \\
\hline 28 & 756 & 750 & 753 & 759 & 752 & 756 & & & & & & \\
\hline 29 & 758 & 754 & 756 & 759 & 752 & 756 & & & & & & \\
\hline 30 & 756 & 753 & 755 & 759 & 751 & 755 & & & & & & \\
\hline 31 & 757 & 752 & 754 & 758 & 754 & 756 & & & & & & \\
\hline Month & -- & -- & -- & 760 & 745 & 754 & & & & & & \\
\hline
\end{tabular}


Table 11. Daily summary of total dissolved gas, in millimeters of mercury, for the Columbia River, right bank, near Cliffs, Washington, March - September 1996

[Max, maximum; Min, minimum; Month, monthly summary statistics; --, not available]

\begin{tabular}{|c|c|c|c|c|c|c|c|c|c|c|c|c|}
\hline \multirow{2}{*}{ Day } & \multicolumn{3}{|c|}{ March } & \multicolumn{3}{|c|}{ April } & \multicolumn{3}{|c|}{ May } & \multicolumn{3}{|c|}{ June } \\
\hline & Max & Min & Mean & Max & Min & Mean & Max & Min & Mean & Max & Min & Mean \\
\hline 1 & & & & 857 & 846 & 852 & 991 & 883 & 924 & 1020 & 903 & 978 \\
\hline 2 & & & & 891 & 841 & 851 & 1020 & 877 & 922 & 1030 & 943 & 976 \\
\hline 3 & & & & 852 & 845 & 849 & 934 & 877 & 903 & 988 & 896 & 951 \\
\hline 4 & & & & 901 & 849 & 868 & 945 & 880 & 917 & 1010 & 902 & 956 \\
\hline 5 & & & & 885 & 852 & 863 & 941 & 880 & 907 & 1020 & 953 & 992 \\
\hline 6 & & & & 905 & 850 & 881 & 929 & 875 & 900 & 1020 & 959 & 991 \\
\hline 7 & & & & 857 & 843 & 848 & 905 & 873 & 892 & 974 & 886 & 942 \\
\hline 8 & & & & 874 & 849 & 857 & 903 & 872 & 882 & 1030 & 923 & 983 \\
\hline 9 & & & & 984 & 848 & 872 & 904 & 853 & 882 & 1030 & 975 & 987 \\
\hline 10 & & & & 1070 & 911 & 978 & 904 & 842 & 864 & 1040 & 996 & 1020 \\
\hline 11 & & & & 1080 & 980 & 1010 & 906 & 850 & 874 & 1030 & 998 & 1020 \\
\hline 12 & & & & 997 & 904 & 935 & 908 & 866 & 883 & 1050 & 992 & 1010 \\
\hline 13 & & & & 939 & 908 & 918 & 897 & 870 & 878 & 998 & 964 & 984 \\
\hline 14 & & & & 917 & 879 & 901 & 924 & 885 & 893 & 1000 & 928 & 978 \\
\hline 15 & & & & 990 & 879 & 929 & 943 & 896 & 915 & 991 & 944 & 966 \\
\hline 16 & & & & 993 & 840 & 909 & 924 & 871 & 899 & 996 & 985 & 992 \\
\hline 17 & & & & 1000 & 841 & 933 & 981 & 869 & 834 & 1000 & 987 & 993 \\
\hline 18 & & & & 999 & 927 & 966 & 1020 & 924 & 977 & 1030 & 980 & 1000 \\
\hline 19 & & & & 945 & 924 & 933 & 1060 & 990 & 1020 & 988 & 867 & 950 \\
\hline 20 & & & & 986 & 903 & 950 & 1060 & 1000 & 1030 & 976 & 850 & 897 \\
\hline 21 & & & & 994 & 883 & 941 & 1020 & 956 & 995 & 887 & 863 & 876 \\
\hline 22 & & & & 935 & 883 & 915 & 992 & 904 & 970 & 907 & 880 & 891 \\
\hline 23 & & & & 1000 & 915 & 954 & 1040 & 914 & 999 & 913 & 874 & 892 \\
\hline 24 & & & & 1080 & 914 & 995 & 1040 & 934 & 965 & 920 & 882 & 890 \\
\hline 25 & & & & 1070 & 972 & 1030 & 988 & 921 & 964 & 897 & 879 & 886 \\
\hline 26 & & & & 1060 & 934 & 989 & 987 & 887 & 940 & 963 & 873 & 901 \\
\hline 27 & & & & 1050 & 934 & 984 & 982 & 876 & 926 & 889 & 863 & 878 \\
\hline 28 & & & & 1080 & 913 & 997 & 1010 & 904 & 948 & 919 & 881 & 896 \\
\hline 29 & & & & 952 & 900 & 931 & 1050 & 934 & 1010 & 902 & 889 & 894 \\
\hline 30 & 855 & 846 & 849 & 939 & 883 & 918 & 1050 & 994 & 1020 & 902 & 884 & 893 \\
\hline 31 & 849 & 844 & 846 & -- & -- & -- & 1020 & 889 & 945 & -- & -- & -- \\
\hline Month & -- & -- & -- & 1080 & 840 & 925 & 1060 & 842 & 932 & 1050 & 850 & 949 \\
\hline \multirow{2}{*}{ Day } & \multicolumn{3}{|c|}{ July } & \multicolumn{3}{|c|}{ August } & \multicolumn{3}{|c|}{ September } & & & \\
\hline & Max & Min & Mean & Max & Min & Mean & Max & Min & Mean & & & \\
\hline 1 & 892 & 843 & 867 & 909 & 827 & 867 & 904 & 776 & 791 & & & \\
\hline 2 & 898 & 856 & 875 & 915 & 814 & 865 & 785 & 773 & 779 & & & \\
\hline 3 & 894 & 875 & 885 & 914 & 801 & 863 & 790 & 771 & 780 & & & \\
\hline 4 & 902 & 880 & 892 & 907 & 787 & 849 & 792 & 770 & 780 & & & \\
\hline 5 & 903 & 843 & 877 & 910 & 778 & 842 & 819 & 769 & 786 & & & \\
\hline 6 & 896 & 879 & 889 & 922 & 772 & 844 & 791 & 773 & 782 & & & \\
\hline 7 & 898 & 838 & 884 & 915 & 776 & 841 & 788 & 772 & 779 & & & \\
\hline 8 & 896 & 842 & 868 & 913 & 773 & 839 & 785 & 769 & 778 & & & \\
\hline 9 & 894 & 851 & 874 & 917 & 777 & 844 & 791 & 774 & 782 & & & \\
\hline 10 & 901 & 840 & 868 & 901 & 784 & 855 & 803 & 774 & 787 & & & \\
\hline 11 & 901 & 837 & 867 & 896 & 787 & 842 & 793 & 774 & 782 & & & \\
\hline 12 & 897 & 841 & 882 & 904 & 792 & 848 & 787 & 772 & 780 & & & \\
\hline 13 & 894 & 840 & 863 & 905 & 801 & 855 & 807 & 768 & 781 & & & \\
\hline 14 & 888 & 859 & 871 & 916 & 799 & 859 & 780 & 767 & 775 & & & \\
\hline 15 & 876 & 850 & 863 & 918 & 809 & 860 & 778 & 765 & 773 & & & \\
\hline 16 & 880 & 821 & 855 & 909 & 808 & 859 & 783 & 766 & 774 & & & \\
\hline 17 & 888 & 820 & 855 & 910 & 803 & 856 & 785 & 763 & 774 & & & \\
\hline 18 & 895 & 808 & 852 & 905 & 798 & 849 & & & & & & \\
\hline 19 & 897 & 796 & 845 & 905 & 795 & 849 & & & & & & \\
\hline 20 & 899 & 795 & 851 & 917 & 790 & 849 & & & & & & \\
\hline 21 & 887 & 795 & 842 & 916 & 793 & 859 & & & & & & \\
\hline 22 & 888 & 801 & 846 & 915 & 792 & 855 & & & & & & \\
\hline 23 & 887 & 804 & 848 & 908 & 797 & 852 & & & & & & \\
\hline 24 & 889 & 815 & 854 & 905 & 804 & 855 & & & & & & \\
\hline 25 & 889 & 831 & 861 & 904 & 811 & 859 & & & & & & \\
\hline 26 & 889 & 852 & 871 & 903 & 817 & 858 & & & & & & \\
\hline 27 & 888 & 855 & 872 & 911 & 818 & 858 & & & & & & \\
\hline 28 & 890 & 850 & 874 & 908 & 807 & 857 & & & & & & \\
\hline 29 & 920 & 838 & 867 & 905 & 804 & 856 & & & & & & \\
\hline 30 & 896 & 833 & 864 & 903 & 796 & 851 & & & & & & \\
\hline 31 & 908 & 829 & 866 & 905 & 788 & 850 & & & & & & \\
\hline Month & 920 & 795 & 866 & 922 & 772 & 853 & & & & & & \\
\hline
\end{tabular}


Table 12. Daily summary of total dissolved gas, in percent saturation, for the Columbia River, right bank, near Cliffs, Washington, March - September 1996

[Max, maximum; Min, minimum; Month, monthly summary statistics; --, not available]

\begin{tabular}{|c|c|c|c|c|c|c|c|c|c|c|c|c|}
\hline \multirow{2}{*}{ Day } & \multicolumn{3}{|c|}{ March } & \multicolumn{3}{|c|}{ April } & \multicolumn{3}{|c|}{ May } & \multicolumn{3}{|c|}{ June } \\
\hline & Max & Min & Mean & Max & Min & Mean & Max & Min & Mean & Max & Min & Mean \\
\hline 1 & & & & 115 & 113 & 115 & 131 & 117 & 122 & 134 & 119 & 128 \\
\hline 2 & & & & 118 & 111 & 113 & 136 & 116 & 122 & -- & -- & -- \\
\hline 3 & & & & 113 & 111 & 112 & 123 & 116 & 119 & -- & -- & -- \\
\hline 4 & & & & 118 & 111 & 114 & 124 & 116 & 121 & -- & -- & -- \\
\hline 5 & & & & 116 & 111 & 113 & 124 & 116 & 120 & -- & -- & -- \\
\hline 6 & & & & 119 & 112 & 116 & 122 & 116 & 119 & -- & -- & -- \\
\hline 7 & & & & 113 & 112 & 112 & 120 & 116 & 118 & -- & -- & -- \\
\hline 8 & & & & 115 & 113 & 114 & 119 & 115 & 116 & -- & -- & -- \\
\hline 9 & & & & 130 & 112 & 115 & 119 & 112 & 116 & -- & -- & -- \\
\hline 10 & & & & 141 & 120 & 129 & 119 & 110 & 114 & -- & -- & -- \\
\hline 11 & & & & 143 & 130 & 134 & 119 & 112 & 115 & -- & -- & -- \\
\hline 12 & & & & 133 & 119 & 124 & 120 & 114 & 116 & -- & -- & -- \\
\hline 13 & & & & 123 & 119 & 121 & 120 & 115 & 117 & -- & -- & -- \\
\hline 14 & & & & 121 & 116 & 119 & 124 & 118 & 119 & -- & -- & -- \\
\hline 15 & & & & 133 & 117 & 124 & 126 & 119 & 122 & -- & -- & -- \\
\hline 16 & & & & 134 & 112 & 122 & 124 & 117 & 120 & -- & -- & -- \\
\hline 17 & & & & 133 & 113 & 125 & 131 & 116 & 125 & -- & -- & -- \\
\hline 18 & & & & 133 & 122 & 128 & 135 & 124 & 130 & -- & -- & -- \\
\hline 19 & & & & 125 & 122 & 123 & 140 & 131 & 134 & -- & -- & -- \\
\hline 20 & & & & 130 & 119 & 125 & 140 & 132 & 136 & -- & -- & -- \\
\hline 21 & & & & 131 & 117 & 124 & 136 & 128 & 133 & -- & -- & -- \\
\hline 22 & & & & 124 & 117 & 121 & 132 & 119 & 128 & 120 & 117 & 118 \\
\hline 23 & & & & 135 & 122 & 127 & 136 & 121 & 131 & 121 & 116 & 118 \\
\hline 24 & & & & 143 & 122 & 132 & 136 & 123 & 127 & 122 & 117 & 118 \\
\hline 25 & & & & 142 & 128 & 136 & 130 & 122 & 127 & 119 & 117 & 118 \\
\hline 26 & & & & 139 & 123 & 130 & 131 & 117 & 124 & -- & -- & -- \\
\hline 27 & & & & 137 & 123 & 129 & 129 & 116 & 122 & -- & -- & -- \\
\hline 28 & & & & 141 & 120 & 130 & 134 & 119 & 125 & -- & -- & -- \\
\hline 29 & & & & 125 & 118 & 122 & 138 & 124 & 133 & -- & -- & -- \\
\hline 30 & 113 & 112 & 113 & 124 & 117 & 121 & 139 & 131 & 135 & -- & -- & -- \\
\hline 31 & 114 & 112 & 113 & -- & -- & -- & 135 & 117 & 125 & -- & -- & -- \\
\hline Month & -- & -- & -- & 143 & 111 & 122 & 140 & 110 & 124 & -- & -- & -- \\
\hline \multirow{2}{*}{ Day } & \multicolumn{3}{|c|}{ July } & \multicolumn{3}{|c|}{ August } & \multicolumn{3}{|c|}{ September } & & & \\
\hline & Max & Min & Mean & Max & Min & Mean & Max & Min & Mean & & & \\
\hline 1 & -- & -- & -- & 121 & 110 & 115 & 120 & 103 & 105 & & & \\
\hline 2 & -- & -- & -- & 122 & 108 & 115 & 104 & 102 & 103 & & & \\
\hline 3 & -- & -- & -- & 121 & 106 & 114 & 105 & 102 & 104 & & & \\
\hline 4 & -- & -- & -- & 121 & 105 & 113 & 105 & 102 & 104 & & & \\
\hline 5 & -- & -- & -- & 121 & 103 & 112 & 108 & 102 & 104 & & & \\
\hline 6 & -- & -- & -- & 122 & 102 & 112 & 105 & 102 & 103 & & & \\
\hline 7 & -- & -- & -- & 121 & 102 & 111 & 104 & 102 & 103 & & & \\
\hline 8 & -- & -- & -- & 121 & 102 & 111 & 104 & 102 & 103 & & & \\
\hline 9 & -- & -- & -- & 121 & 103 & 112 & 105 & 102 & 104 & & & \\
\hline 10 & -- & -- & -- & 120 & 105 & 114 & 106 & 103 & 104 & & & \\
\hline 11 & -- & -- & -- & 120 & 105 & 112 & 106 & 103 & 104 & & & \\
\hline 12 & -- & -- & -- & 120 & 104 & 112 & 105 & 103 & 104 & & & \\
\hline 13 & -- & -- & -- & 120 & 106 & 114 & 108 & 103 & 104 & & & \\
\hline 14 & -- & -- & -- & 122 & 106 & 114 & 105 & 102 & 104 & & & \\
\hline 15 & -- & -- & -- & 122 & 107 & 114 & 104 & 102 & 103 & & & \\
\hline 16 & -- & -- & -- & 121 & 107 & 114 & 104 & 102 & 103 & & & \\
\hline 17 & -- & -- & -- & 121 & 107 & 114 & 103 & 101 & 102 & & & \\
\hline 18 & -- & -- & -- & 120 & 105 & 112 & & & & & & \\
\hline 19 & -- & -- & -- & 120 & 106 & 113 & & & & & & \\
\hline 20 & -- & -- & -- & 121 & 104 & 113 & & & & & & \\
\hline 21 & 118 & 105 & 112 & 121 & 104 & 113 & & & & & & \\
\hline 22 & 118 & 106 & 112 & 121 & 105 & 113 & & & & & & \\
\hline 23 & 118 & 106 & 112 & 120 & 105 & 113 & & & & & & \\
\hline 24 & 118 & 108 & 114 & 120 & 106 & 113 & & & & & & \\
\hline 25 & 119 & 110 & 115 & 120 & 108 & 114 & & & & & & \\
\hline 26 & 119 & 113 & 116 & 121 & 109 & 115 & & & & & & \\
\hline 27 & 118 & 114 & 116 & 121 & 109 & 114 & & & & & & \\
\hline 28 & 118 & 113 & 116 & 121 & 107 & 113 & & & & & & \\
\hline 29 & 122 & 111 & 115 & 120 & 106 & 113 & & & & & & \\
\hline 30 & 119 & 111 & 115 & 120 & 105 & 113 & & & & & & \\
\hline 31 & 120 & 109 & 115 & 120 & 104 & 112 & & & & & & \\
\hline Month & -- & -- & -- & 122 & 102 & 113 & & & & & & \\
\hline
\end{tabular}



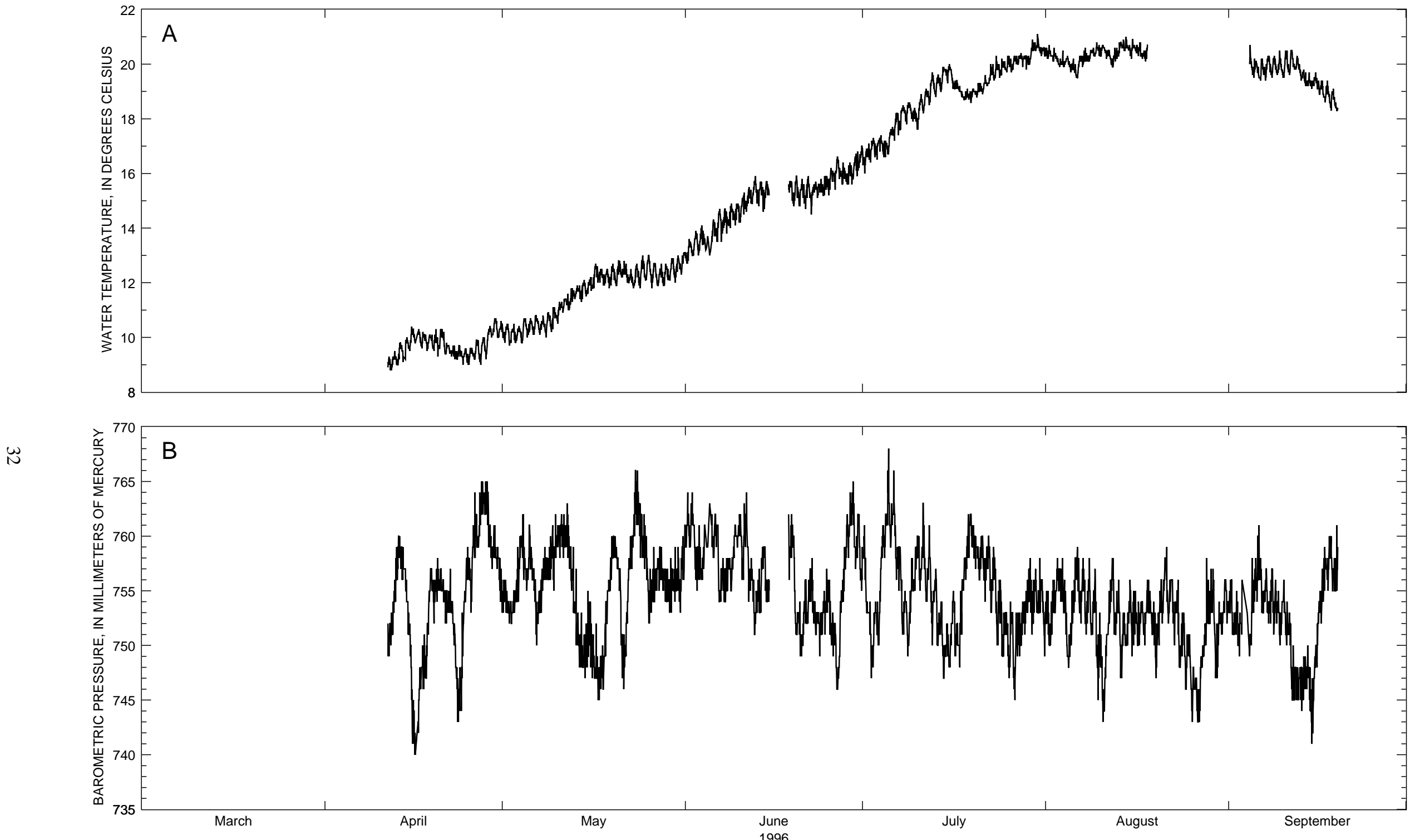

Figure 7. Hourly values of (A) water temperature, (B) barometric pressure, and (C, D) total dissolved gas at the Columbia River at The Dalles Dam forebay, Washington, April - September 1996. 

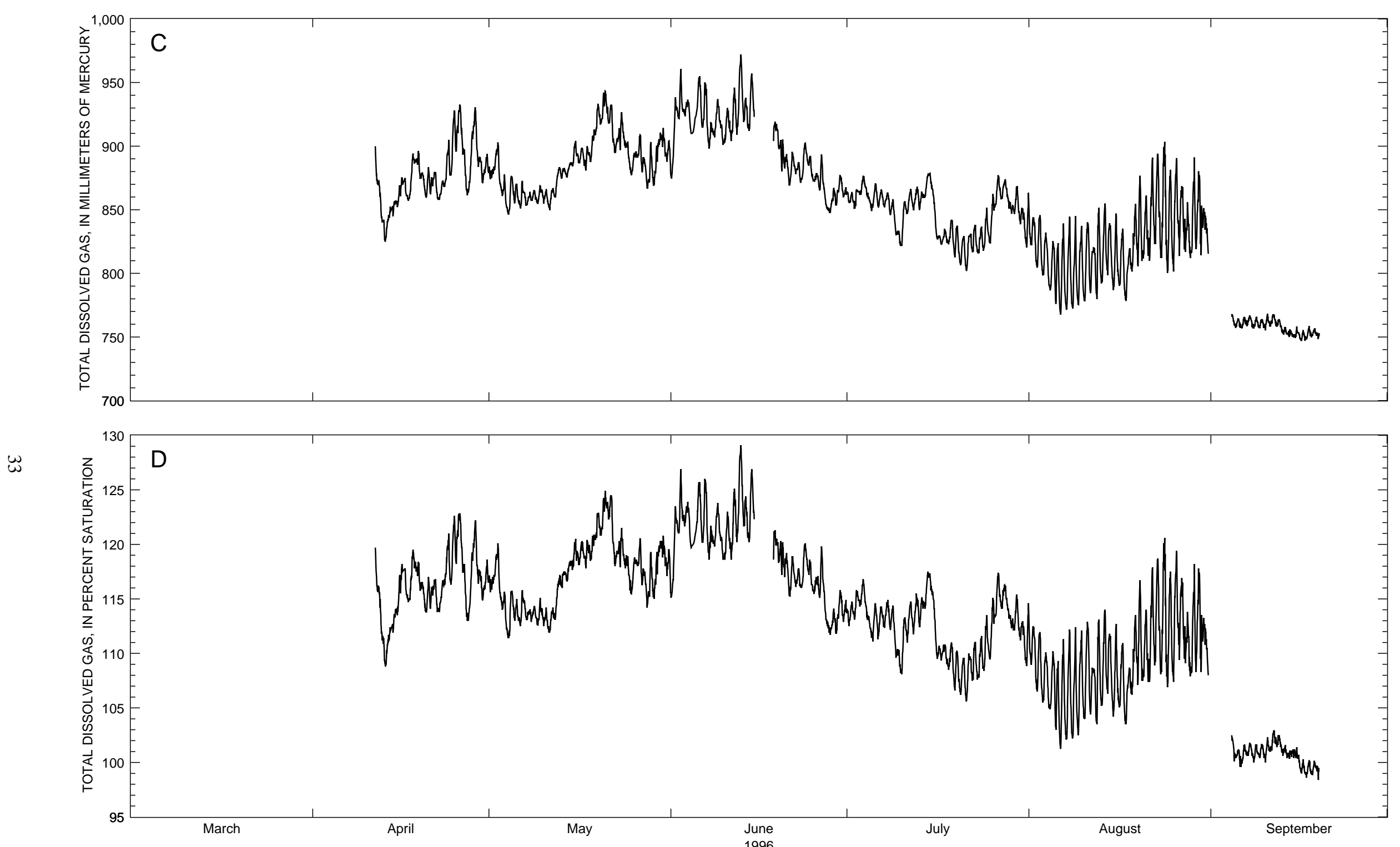

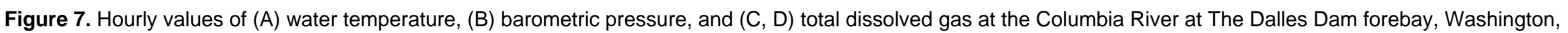
April - September 1996.—Continued 
Table 39. Daily summary of total dissolved gas, in millimeters of mercury, for the Columbia River, right bank, near Washougal, Washington, March - September 1996

[Max, maximum; Min, minimum; Month, monthly summary statistics; --, not available]

\begin{tabular}{|c|c|c|c|c|c|c|c|c|c|c|c|c|}
\hline \multirow{2}{*}{ Day } & \multicolumn{3}{|c|}{ March } & \multicolumn{3}{|c|}{ April } & \multicolumn{3}{|c|}{ May } & \multicolumn{3}{|c|}{ June } \\
\hline & Max & Min & Mean & Max & Min & Mean & Max & Min & Mean & Max & Min & Mean \\
\hline 1 & -- & -- & -- & 865 & 844 & 855 & 949 & 922 & 940 & 1070 & 938 & 986 \\
\hline 2 & -- & -- & -- & 867 & 848 & 860 & 931 & 917 & 923 & 1050 & 962 & 999 \\
\hline 3 & -- & -- & -- & 899 & 857 & 877 & 958 & 912 & 928 & 993 & 957 & 971 \\
\hline 4 & -- & -- & -- & 889 & 866 & 878 & 948 & 907 & 920 & 975 & 937 & 953 \\
\hline 5 & -- & -- & -- & 901 & 870 & 886 & 968 & 912 & 933 & 961 & 930 & 945 \\
\hline 6 & -- & -- & -- & 921 & 887 & 901 & 977 & 920 & 936 & 996 & 950 & 975 \\
\hline 7 & -- & -- & -- & 940 & 894 & 912 & 928 & 901 & 916 & 979 & 949 & 964 \\
\hline 8 & -- & -- & -- & 929 & 899 & 913 & 920 & 893 & 907 & 971 & 940 & 956 \\
\hline 9 & 931 & 870 & 892 & 902 & 885 & 897 & 944 & 900 & 923 & 999 & 941 & 966 \\
\hline 10 & 973 & 901 & 931 & 909 & 863 & 881 & 931 & 909 & 919 & 1010 & 963 & 981 \\
\hline 11 & 977 & 915 & 950 & 922 & 876 & 897 & 917 & 893 & 908 & 979 & 964 & 972 \\
\hline 12 & 978 & 934 & 957 & 932 & 920 & 927 & 904 & 879 & 892 & 987 & 968 & 976 \\
\hline 13 & 981 & 922 & 946 & 954 & 926 & 939 & 936 & 895 & 914 & 1000 & 943 & 969 \\
\hline 14 & 978 & 955 & 969 & 939 & 906 & 920 & 948 & 906 & 923 & 1030 & 936 & 966 \\
\hline 15 & 987 & 966 & 976 & 943 & 912 & 926 & 948 & 902 & 922 & 1010 & 927 & 956 \\
\hline 16 & 978 & 928 & 959 & 964 & 910 & 937 & 953 & 922 & 935 & 1040 & 926 & 976 \\
\hline 17 & 1000 & 929 & 961 & 919 & 902 & 910 & 939 & 922 & 931 & 1020 & 928 & 954 \\
\hline 18 & 950 & 908 & 935 & 918 & 904 & 911 & 933 & 916 & 924 & 1040 & -- & -- \\
\hline 19 & 951 & 906 & 930 & 932 & 910 & 921 & 986 & 914 & 945 & -- & -- & -- \\
\hline 20 & 949 & 910 & 927 & 942 & 920 & 932 & 1050 & 939 & 980 & 989 & 941 & 965 \\
\hline 21 & 939 & 903 & 919 & 939 & 916 & 929 & 1030 & 950 & 980 & 959 & 916 & 941 \\
\hline 22 & 928 & 901 & 917 & 924 & 911 & 919 & 963 & 928 & 946 & 929 & 889 & 906 \\
\hline 23 & 918 & 891 & 905 & 930 & 908 & 920 & 933 & 921 & 927 & 947 & 877 & 903 \\
\hline 24 & 901 & 866 & 887 & 929 & 903 & 914 & 1000 & 926 & 961 & 947 & 883 & 904 \\
\hline 25 & 890 & 859 & 874 & 956 & 924 & 937 & 1020 & 950 & 995 & 1020 & 904 & 945 \\
\hline 26 & 922 & 863 & 897 & 963 & 941 & 951 & 987 & 930 & 958 & 1040 & 915 & 963 \\
\hline 27 & 910 & 881 & 896 & 969 & 939 & 954 & 962 & 909 & 935 & 979 & 901 & 929 \\
\hline 28 & 894 & 877 & 886 & 951 & 935 & 945 & -- & -- & -- & 929 & 899 & 910 \\
\hline 29 & 888 & 859 & 872 & 985 & 947 & 964 & -- & -- & -- & 928 & 896 & 910 \\
\hline 30 & 882 & 859 & 870 & 970 & 927 & 958 & -- & -- & -- & 924 & 897 & 911 \\
\hline 31 & 879 & 851 & 861 & -- & -- & -- & -- & -- & -- & -- & -- & -- \\
\hline Month & -- & -- & -- & 985 & 844 & 916 & -- & -- & -- & -- & -- & -- \\
\hline \multirow{2}{*}{ Day } & \multicolumn{3}{|c|}{ July } & \multicolumn{3}{|c|}{ August } & \multicolumn{3}{|c|}{ September } & & & \\
\hline & Max & Min & Mean & Max & Min & Mean & Max & Min & Mean & & & \\
\hline 1 & 939 & 910 & 921 & 887 & 844 & 863 & 872 & 830 & 852 & & & \\
\hline 2 & 913 & 889 & 899 & 862 & 832 & 844 & 830 & 796 & 808 & & & \\
\hline 3 & 901 & 868 & 881 & 889 & 837 & 857 & 810 & 797 & 803 & & & \\
\hline 4 & 887 & 857 & 870 & 885 & 847 & 862 & 808 & 792 & 799 & & & \\
\hline 5 & 905 & 863 & 884 & 880 & 842 & 859 & 803 & 787 & 794 & & & \\
\hline 6 & 911 & 872 & 890 & 901 & 835 & 862 & 803 & 787 & 795 & & & \\
\hline 7 & 906 & 887 & 897 & 899 & 840 & 865 & 810 & 787 & 797 & & & \\
\hline 8 & 923 & 886 & 901 & 922 & 861 & 886 & 803 & 786 & 796 & & & \\
\hline 9 & 892 & 867 & 879 & 917 & 877 & 893 & 786 & 769 & 778 & & & \\
\hline 10 & 872 & 841 & 859 & 919 & 874 & 892 & 781 & 761 & 772 & & & \\
\hline 11 & 903 & 862 & 880 & 904 & 862 & 879 & 781 & 763 & 772 & & & \\
\hline 12 & 918 & 873 & 894 & 907 & 854 & 876 & 775 & 751 & 764 & & & \\
\hline 13 & 922 & 891 & 905 & 893 & 845 & 867 & 767 & 753 & 761 & & & \\
\hline 14 & 906 & 871 & 889 & 896 & 842 & 866 & 760 & 752 & 756 & & & \\
\hline 15 & 914 & 877 & 892 & 883 & 826 & 847 & 759 & 744 & 752 & & & \\
\hline 16 & 889 & 849 & 868 & 867 & 824 & 843 & 768 & 746 & 758 & & & \\
\hline 17 & 849 & 825 & 836 & 877 & 819 & 844 & 771 & 751 & 762 & & & \\
\hline 18 & 876 & 826 & 849 & 879 & 821 & 848 & 771 & 756 & 763 & & & \\
\hline 19 & 908 & 854 & 876 & 896 & 837 & 864 & & & & & & \\
\hline 20 & 897 & 865 & 879 & 885 & 825 & 850 & & & & & & \\
\hline 21 & 906 & 857 & 877 & 894 & 837 & 864 & & & & & & \\
\hline 22 & 925 & 870 & 888 & 894 & 843 & 865 & & & & & & \\
\hline 23 & 917 & 870 & 889 & 894 & 836 & 861 & & & & & & \\
\hline 24 & 927 & 879 & 899 & 903 & 850 & 872 & & & & & & \\
\hline 25 & 922 & 884 & 899 & 897 & 856 & 876 & & & & & & \\
\hline 26 & 925 & 877 & 896 & 889 & 847 & 865 & & & & & & \\
\hline 27 & 912 & 881 & 894 & 875 & 846 & 859 & & & & & & \\
\hline 28 & 903 & 884 & 890 & 892 & 843 & 864 & & & & & & \\
\hline 29 & 925 & 877 & 894 & 885 & 835 & 859 & & & & & & \\
\hline 30 & 899 & 865 & 878 & 880 & 845 & 861 & & & & & & \\
\hline 31 & 896 & 845 & 865 & 883 & 849 & 865 & & & & & & \\
\hline Month & 939 & 825 & 884 & 922 & 819 & 864 & & & & & & \\
\hline
\end{tabular}


Table 14. Daily summary of barometric pressure for the Columbia River at The Dalles Dam forebay, Washington, April - September, 1996

[Values reported in millimeters of mercury; Max, maximum; Min, minimum; Month, monthly summary statistics; --, not available; e, estimated]

\begin{tabular}{|c|c|c|c|c|c|c|c|c|c|c|c|c|}
\hline \multirow{2}{*}{ Day } & \multicolumn{3}{|c|}{ April } & \multicolumn{3}{|c|}{ May } & \multicolumn{3}{|c|}{ June } & \multicolumn{3}{|c|}{ July } \\
\hline & Max & Min & Mean & Max & Min & Mean & Max & Min & Mean & Max & Min & Mean \\
\hline 1 & -- & -- & -- & 756 & 753 & 754 & 764 & 759 & 761 & 761 & 753 & 756 \\
\hline 2 & -- & -- & -- & 755 & 752 & 753 & 764 & 755 & 760 & 754 & 747 & 751 \\
\hline 3 & -- & -- & -- & 760 & 753 & 756 & 761 & 755 & 758 & 755 & 750 & 752 \\
\hline 4 & -- & -- & -- & 762 & 756 & 759 & -- & -- & -- & 762 & 754 & 759 \\
\hline 5 & -- & -- & -- & 761 & 755 & 758 & -- & -- & -- & 768 & 759 & 762 \\
\hline 6 & -- & -- & -- & 758 & 750 & 755 & 762 & 754 & 758 & 766 & 756 & 760 \\
\hline 7 & -- & -- & -- & 758 & 752 & 755 & 758 & 754 & 756 & 759 & 751 & 756 \\
\hline 8 & -- & -- & -- & 759 & 756 & 757 & 758 & 755 & 757 & 755 & 749 & 752 \\
\hline 9 & -- & -- & -- & 761 & 755 & 758 & 761 & 757 & 760 & 759 & 752 & 756 \\
\hline 10 & -- & -- & -- & 762 & 758 & 760 & 763 & 756 & 760 & 760 & 755 & 758 \\
\hline 11 & -- & -- & -- & 763 & 758 & 760 & 764 & 754 & 759 & 763 & 754 & 758 \\
\hline 12 & 758 & 750 & 753 & 763 & 755 & 758 & 758 & 751 & 754 & 761 & 750 & 756 \\
\hline 13 & 760 & 756 & 758 & 759 & 750 & 753 & 758 & 753 & 755 & 757 & 750 & 754 \\
\hline 14 & -- & -- & -- & 753 & 748 & 749 & 759 & 754 & 757 & 754 & 747 & 751 \\
\hline 15 & 754 & 741 & 748 & 755 & 747 & 751 & -- & -- & -- & 752 & 748 & 750 \\
\hline 16 & -- & -- & -- & 752 & 747 & 749 & -- & -- & -- & 755 & 749 & 752 \\
\hline 17 & 751 & 746 & 748 & 750 & 745 & 748 & -- & -- & -- & 756 & e748 & 752 \\
\hline 18 & 757 & 747 & 752 & 755 & 746 & 751 & -- & -- & -- & 762 & 755 & 758 \\
\hline 19 & 757 & 752 & 755 & 760 & 755 & 757 & 762 & 751 & 756 & 762 & 758 & 760 \\
\hline 20 & 758 & 755 & 756 & 760 & 755 & 758 & 754 & 749 & 751 & 760 & 756 & 758 \\
\hline 21 & 756 & 752 & 754 & 755 & 746 & 750 & 756 & 751 & 754 & 760 & 754 & 758 \\
\hline 22 & 757 & 750 & 753 & 760 & 752 & 756 & 758 & 752 & 755 & 760 & 753 & 757 \\
\hline 23 & 751 & 743 & 747 & 766 & 759 & 762 & 753 & 749 & 752 & 759 & 750 & 755 \\
\hline 24 & 758 & 744 & 752 & 764 & 758 & 761 & 757 & 751 & 753 & 756 & 749 & 752 \\
\hline 25 & 759 & 753 & 757 & 762 & 752 & 757 & 756 & 750 & 753 & 753 & 747 & 751 \\
\hline 26 & 764 & 758 & 760 & 759 & 753 & 755 & 752 & 746 & 749 & 754 & 745 & 750 \\
\hline 27 & 765 & 759 & 762 & 759 & 755 & 757 & 759 & 748 & 754 & 753 & 749 & 751 \\
\hline 28 & 765 & 759 & 762 & 757 & 754 & 756 & 764 & 757 & 760 & 756 & 751 & 753 \\
\hline 29 & 761 & 757 & 759 & 758 & 753 & 756 & 765 & 757 & 761 & 758 & 751 & 754 \\
\hline 30 & 759 & 753 & 757 & 759 & 754 & 756 & 762 & 755 & 759 & 757 & 753 & 754 \\
\hline 31 & -- & -- & -- & 761 & 753 & 757 & -- & -- & -- & 758 & 749 & 754 \\
\hline Month & -- & -- & -- & 766 & 745 & 756 & -- & -- & -- & 768 & 745 & 755 \\
\hline
\end{tabular}

\begin{tabular}{|c|c|c|c|c|c|c|}
\hline \multirow{2}{*}{ Day } & \multicolumn{3}{|c|}{ August } & \multicolumn{3}{|c|}{ September } \\
\hline & Max & Min & Mean & Max & Min & Mean \\
\hline 1 & 755 & 750 & 752 & 756 & 751 & 753 \\
\hline 2 & 758 & 751 & 754 & -- & -- & -- \\
\hline 3 & 758 & 754 & 756 & -- & -- & -- \\
\hline 4 & 756 & 748 & 752 & -- & -- & -- \\
\hline 5 & 758 & 749 & 753 & 760 & 753 & 756 \\
\hline 6 & 759 & 752 & 756 & 761 & 753 & 756 \\
\hline 7 & 758 & 751 & 754 & 757 & 752 & 754 \\
\hline 8 & 758 & 751 & 753 & 757 & 751 & 754 \\
\hline 9 & 757 & 745 & 752 & 755 & 750 & 753 \\
\hline 10 & 753 & 743 & 747 & 756 & 750 & 753 \\
\hline 11 & 756 & 746 & 751 & 753 & 745 & 749 \\
\hline 12 & 758 & 751 & 754 & 748 & 745 & 747 \\
\hline 13 & 756 & 747 & 752 & 749 & 744 & 747 \\
\hline 14 & 753 & 750 & 751 & -- & -- & -- \\
\hline 15 & 756 & 749 & 753 & -- & -- & -- \\
\hline 16 & 755 & 750 & 752 & 757 & 750 & 754 \\
\hline 17 & e754 & 750 & 753 & & & \\
\hline 18 & e756 & 752 & 754 & & & \\
\hline 19 & 754 & 747 & 751 & & & \\
\hline 20 & 756 & 750 & 753 & & & \\
\hline 21 & 759 & 752 & 755 & & & \\
\hline 22 & 756 & 750 & 754 & & & \\
\hline 23 & 756 & 748 & 751 & & & \\
\hline 24 & 753 & 746 & 750 & & & \\
\hline 25 & 751 & 743 & 748 & & & \\
\hline 26 & 748 & 743 & 746 & & & \\
\hline 27 & 752 & 743 & 748 & & & \\
\hline 28 & 758 & 750 & 753 & & & \\
\hline 29 & 757 & 747 & 752 & & & \\
\hline 30 & 754 & 747 & 752 & & & \\
\hline 31 & 756 & 752 & 754 & & & \\
\hline Month & 759 & 743 & 752 & & & \\
\hline
\end{tabular}


Table 15. Daily summary of total dissolved gas, in millimeters of mercury, for the Columbia River at The Dalles Dam forebay, Washington, April - September 1996

[Max, maximum; Min, minimum; Month, monthly summary statistics; --, not available]

\begin{tabular}{|c|c|c|c|c|c|c|c|c|c|c|c|c|}
\hline \multirow{2}{*}{ Day } & \multicolumn{3}{|c|}{ April } & \multicolumn{3}{|c|}{ May } & \multicolumn{3}{|c|}{ June } & \multicolumn{3}{|c|}{ July } \\
\hline & Max & Min & Mean & Max & Min & Mean & Max & Min & Mean & Max & Min & Mean \\
\hline 1 & -- & -- & -- & 884 & 874 & 880 & 938 & 875 & 904 & 864 & 855 & 860 \\
\hline 2 & -- & -- & -- & 903 & 870 & 888 & 961 & 922 & 935 & 866 & 851 & 859 \\
\hline 3 & -- & -- & -- & 878 & 853 & 865 & 936 & 924 & 930 & 876 & 862 & 868 \\
\hline 4 & -- & -- & -- & 877 & 847 & 859 & -- & -- & -- & 870 & 855 & 862 \\
\hline 5 & -- & -- & -- & 873 & 855 & 864 & 955 & -- & -- & 871 & 849 & 859 \\
\hline 6 & -- & -- & -- & 872 & 851 & 860 & 950 & 915 & 931 & 866 & 852 & 859 \\
\hline 7 & -- & -- & -- & 864 & 853 & 858 & 945 & 898 & 914 & 863 & 852 & 858 \\
\hline 8 & -- & -- & -- & 865 & 857 & 861 & 937 & 907 & 917 & 861 & 846 & 853 \\
\hline 9 & -- & -- & -- & 869 & 855 & 861 & 937 & 901 & 917 & 849 & 823 & 834 \\
\hline 10 & -- & -- & -- & 866 & 855 & 861 & 930 & 902 & 916 & 857 & 821 & 838 \\
\hline 11 & -- & -- & -- & 867 & 850 & 859 & 946 & 904 & 922 & 866 & 847 & 856 \\
\hline 12 & 873 & 840 & 858 & 883 & 860 & 870 & 972 & 909 & 937 & 868 & 850 & 858 \\
\hline 13 & 846 & 825 & 836 & 883 & 874 & 880 & 966 & 919 & 934 & 866 & 850 & 859 \\
\hline 14 & -- & -- & -- & 887 & 875 & 881 & 957 & 912 & 932 & 878 & 859 & 868 \\
\hline 15 & 869 & 852 & 859 & 904 & 884 & 893 & -- & -- & -- & 879 & 848 & 866 \\
\hline 16 & -- & -- & -- & 899 & 887 & 893 & -- & -- & -- & 848 & 824 & 831 \\
\hline 17 & 889 & 857 & 866 & 900 & 881 & 891 & -- & -- & -- & 835 & 823 & 828 \\
\hline 18 & 896 & 885 & 890 & 913 & 887 & 898 & -- & -- & -- & 842 & 823 & 831 \\
\hline 19 & 896 & 868 & 879 & 933 & 909 & 921 & 917 & 880 & 901 & 837 & 813 & 826 \\
\hline 20 & 883 & 859 & 869 & 943 & 917 & 931 & 905 & 879 & 890 & 829 & 806 & 818 \\
\hline 21 & 880 & 863 & 873 & 933 & 918 & 926 & 895 & 869 & 882 & 830 & 802 & 816 \\
\hline 22 & 871 & 858 & 862 & 920 & 895 & 903 & 887 & 875 & 883 & 838 & 817 & 827 \\
\hline 23 & 895 & 868 & 876 & 927 & 897 & 912 & 902 & 872 & 885 & 837 & 816 & 824 \\
\hline 24 & 921 & 877 & 892 & 908 & 885 & 898 & 898 & 878 & 889 & 852 & 818 & 832 \\
\hline 25 & 928 & 896 & 915 & 895 & 877 & 886 & 879 & 872 & 875 & 862 & 823 & 839 \\
\hline 26 & 932 & 877 & 906 & 909 & 887 & 897 & 893 & 865 & 876 & 877 & 849 & 863 \\
\hline 27 & 892 & 861 & 871 & 891 & 867 & 883 & 871 & 849 & 856 & 874 & 858 & 866 \\
\hline 28 & 931 & 892 & 914 & 903 & 867 & 881 & 867 & 847 & 857 & 873 & 849 & 859 \\
\hline 29 & 897 & 871 & 886 & 905 & 869 & 887 & 877 & 853 & 865 & 869 & 847 & 854 \\
\hline 30 & 894 & 873 & 883 & 914 & 896 & 906 & 874 & 860 & 865 & 869 & 838 & 849 \\
\hline 31 & -- & -- & -- & 904 & 877 & 894 & -- & -- & -- & 863 & 820 & 838 \\
\hline Month & -- & -- & -- & 943 & 847 & 885 & -- & -- & -- & 879 & 802 & 847 \\
\hline \multirow{2}{*}{ Day } & \multicolumn{3}{|c|}{ August } & \multicolumn{3}{|c|}{ September } & & & & & & \\
\hline & Max & Min & Mean & Max & Min & Mean & & & & & & \\
\hline 1 & 851 & 822 & 834 & -- & -- & -- & & & & & & \\
\hline 2 & 846 & 805 & 824 & -- & -- & -- & & & & & & \\
\hline 3 & 832 & 798 & 814 & -- & -- & -- & & & & & & \\
\hline 4 & 824 & 787 & 801 & -- & -- & -- & & & & & & \\
\hline 5 & 825 & 776 & 808 & 764 & 757 & 761 & & & & & & \\
\hline 6 & 839 & 767 & 799 & 765 & 757 & 761 & & & & & & \\
\hline 7 & 845 & 772 & 800 & 767 & 758 & 763 & & & & & & \\
\hline 8 & 845 & 772 & 800 & 766 & 757 & 761 & & & & & & \\
\hline 9 & 837 & 776 & 803 & 764 & 757 & 760 & & & & & & \\
\hline 10 & 840 & 778 & 803 & 768 & 755 & 761 & & & & & & \\
\hline 11 & 840 & 784 & 810 & 767 & 758 & 764 & & & & & & \\
\hline 12 & 851 & 780 & 814 & 764 & 758 & 761 & & & & & & \\
\hline 13 & 855 & 792 & 818 & 758 & 752 & 755 & & & & & & \\
\hline 14 & 850 & 799 & 823 & 756 & 750 & 753 & & & & & & \\
\hline 15 & 847 & 787 & 810 & 758 & 749 & 753 & & & & & & \\
\hline 16 & 835 & 790 & 809 & 755 & 747 & 750 & & & & & & \\
\hline 17 & 834 & 778 & 798 & 759 & 747 & 752 & & & & & & \\
\hline 18 & 844 & 801 & 815 & 757 & 750 & 753 & & & & & & \\
\hline 19 & 877 & 806 & 839 & & & & & & & & & \\
\hline 20 & 865 & 810 & 832 & & & & & & & & & \\
\hline 21 & 889 & 810 & 837 & & & & & & & & & \\
\hline 22 & 894 & 823 & 858 & & & & & & & & & \\
\hline 23 & 899 & 812 & 846 & & & & & & & & & \\
\hline 24 & 903 & 800 & 845 & & & & & & & & & \\
\hline 25 & 881 & 801 & 841 & & & & & & & & & \\
\hline 26 & 891 & 814 & 852 & & & & & & & & & \\
\hline 27 & 869 & 817 & 840 & & & & & & & & & \\
\hline 28 & 869 & 812 & 831 & & & & & & & & & \\
\hline 29 & 891 & 819 & 853 & & & & & & & & & \\
\hline 30 & 877 & 814 & 844 & & & & & & & & & \\
\hline 31 & -- & -- & -- & & & & & & & & & \\
\hline Month & -- & -- & -- & & & & & & & & & \\
\hline
\end{tabular}


Table 16. Daily summary of total dissolved gas, in percent saturation, for the Columbia River at The Dalles Dam forebay, Washington, April - September 1996

[Max, maximum; Min, minimum; Month, monthly summary statistics; --, not available]

\begin{tabular}{|c|c|c|c|c|c|c|c|c|c|c|c|c|}
\hline \multirow{2}{*}{ Day } & \multicolumn{3}{|c|}{ April } & \multicolumn{3}{|c|}{ May } & \multicolumn{3}{|c|}{ June } & \multicolumn{3}{|c|}{ July } \\
\hline & Max & Min & Mean & Max & Min & Mean & Max & Min & Mean & Max & Min & Mean \\
\hline 1 & -- & -- & -- & 117 & 116 & 117 & 123 & 115 & 119 & 115 & 112 & 114 \\
\hline 2 & -- & -- & -- & 120 & 115 & 118 & 127 & 121 & 123 & 116 & 113 & 114 \\
\hline 3 & -- & -- & -- & 116 & 112 & 114 & 124 & 122 & 123 & 117 & 114 & 115 \\
\hline 4 & -- & -- & -- & 116 & 111 & 113 & -- & -- & -- & 115 & 112 & 114 \\
\hline 5 & -- & -- & -- & 115 & 113 & 114 & -- & -- & -- & 115 & 111 & 113 \\
\hline 6 & -- & -- & -- & 116 & 112 & 114 & 126 & 120 & 123 & 114 & 111 & 113 \\
\hline 7 & -- & -- & -- & 115 & 113 & 114 & 125 & 119 & 121 & 115 & 112 & 113 \\
\hline 8 & -- & -- & -- & 114 & 113 & 114 & 124 & 120 & 121 & 114 & 112 & 113 \\
\hline 9 & -- & -- & -- & 115 & 113 & 114 & 124 & 119 & 121 & 113 & 109 & 110 \\
\hline 10 & -- & -- & -- & 114 & 112 & 113 & 123 & 119 & 121 & 113 & 108 & 111 \\
\hline 11 & -- & -- & -- & 114 & 112 & 113 & 125 & 119 & 121 & 115 & 111 & 113 \\
\hline 12 & 116 & 111 & 114 & 117 & 113 & 115 & 129 & 120 & 124 & 115 & 112 & 114 \\
\hline 13 & 112 & 109 & 110 & 117 & 116 & 117 & 128 & 122 & 124 & 115 & 112 & 114 \\
\hline 14 & -- & -- & -- & 118 & 117 & 118 & 127 & 120 & 123 & 117 & 114 & 116 \\
\hline 15 & 117 & 113 & 115 & 120 & 118 & 119 & -- & -- & -- & 117 & 113 & 116 \\
\hline 16 & -- & -- & -- & 120 & 118 & 119 & -- & -- & -- & 113 & 109 & 110 \\
\hline 17 & 119 & 115 & 116 & 120 & 118 & 119 & -- & -- & -- & 111 & 109 & 110 \\
\hline 18 & 119 & 117 & 118 & 121 & 118 & 120 & -- & -- & -- & 111 & 108 & 110 \\
\hline 19 & 118 & 115 & 116 & 123 & 120 & 122 & 120 & 117 & 119 & 110 & 107 & 109 \\
\hline 20 & 117 & 114 & 115 & 125 & 121 & 123 & 120 & 117 & 118 & 110 & 106 & 108 \\
\hline 21 & 117 & 114 & 116 & 124 & 122 & 123 & 119 & 115 & 117 & 110 & 106 & 108 \\
\hline 22 & 116 & 114 & 115 & 122 & 118 & 119 & 118 & 116 & 117 & 111 & 107 & 109 \\
\hline 23 & 120 & 116 & 117 & 121 & 118 & 120 & 120 & 116 & 118 & 112 & 108 & 109 \\
\hline 24 & 122 & 116 & 119 & 119 & 116 & 118 & 120 & 116 & 118 & 113 & 108 & 111 \\
\hline 25 & 123 & 118 & 121 & 119 & 115 & 117 & 117 & 115 & 116 & 115 & 109 & 112 \\
\hline 26 & 123 & 116 & 119 & 120 & 117 & 119 & 120 & 115 & 117 & 117 & 113 & 115 \\
\hline 27 & 117 & 113 & 114 & 118 & 114 & 117 & 116 & 112 & 114 & 116 & 114 & 115 \\
\hline 28 & 122 & 117 & 120 & 119 & 114 & 117 & 114 & 112 & 113 & 116 & 112 & 114 \\
\hline 29 & 118 & 115 & 117 & 120 & 115 & 117 & 116 & 112 & 114 & 115 & 112 & 113 \\
\hline 30 & 119 & 115 & 117 & 121 & 119 & 120 & 115 & 113 & 114 & 115 & 111 & 113 \\
\hline 31 & -- & -- & -- & 119 & 115 & 118 & -- & -- & -- & 115 & 109 & 111 \\
\hline Month & -- & -- & -- & 125 & 111 & 117 & -- & -- & -- & 117 & 106 & 112 \\
\hline \multirow{2}{*}{ Day } & \multicolumn{3}{|c|}{ August } & \multicolumn{3}{|c|}{ September } & & & & & & \\
\hline & Max & Min & Mean & Max & Min & Mean & & & & & & \\
\hline 1 & 113 & 109 & 111 & -- & -- & -- & & & & & & \\
\hline 2 & 112 & 106 & 109 & -- & -- & -- & & & & & & \\
\hline 3 & 110 & 105 & 108 & -- & -- & -- & & & & & & \\
\hline 4 & 109 & 105 & 107 & -- & -- & -- & & & & & & \\
\hline 5 & 110 & 103 & 107 & 101 & 100 & 101 & & & & & & \\
\hline 6 & 111 & 101 & 106 & 102 & 100 & 101 & & & & & & \\
\hline 7 & 112 & 102 & 106 & 102 & 101 & 101 & & & & & & \\
\hline 8 & 112 & 102 & 106 & 102 & 100 & 101 & & & & & & \\
\hline 9 & 112 & 102 & 107 & 102 & 100 & 101 & & & & & & \\
\hline 10 & 113 & 104 & 107 & 102 & 100 & 101 & & & & & & \\
\hline 11 & 113 & 104 & 108 & 103 & 101 & 102 & & & & & & \\
\hline 12 & 113 & 103 & 108 & 102 & 101 & 102 & & & & & & \\
\hline 13 & 114 & 105 & 109 & 102 & 100 & 101 & & & & & & \\
\hline 14 & 113 & 106 & 110 & 101 & 100 & 101 & & & & & & \\
\hline 15 & 113 & 104 & 108 & 101 & 100 & 101 & & & & & & \\
\hline 16 & 111 & 105 & 108 & 100 & 99 & 100 & & & & & & \\
\hline 17 & 111 & 103 & 106 & 100 & 99 & 99 & & & & & & \\
\hline 18 & 112 & 106 & 108 & 100 & 99 & 99 & & & & & & \\
\hline 19 & 117 & 107 & 112 & & & & & & & & & \\
\hline 20 & 115 & 107 & 110 & & & & & & & & & \\
\hline 21 & 118 & 107 & 111 & & & & & & & & & \\
\hline 22 & 119 & 109 & 114 & & & & & & & & & \\
\hline 23 & 120 & 108 & 113 & & & & & & & & & \\
\hline 24 & 121 & 107 & 113 & & & & & & & & & \\
\hline 25 & 117 & 107 & 113 & & & & & & & & & \\
\hline 26 & 119 & 109 & 114 & & & & & & & & & \\
\hline 27 & 117 & 109 & 112 & & & & & & & & & \\
\hline 28 & 115 & 108 & 110 & & & & & & & & & \\
\hline 29 & 118 & 108 & 113 & & & & & & & & & \\
\hline 30 & 117 & 108 & 112 & & & & & & & & & \\
\hline 31 & -- & -- & -- & & & & & & & & & \\
\hline Month & -- & -- & -- & & & & & & & & & \\
\hline
\end{tabular}



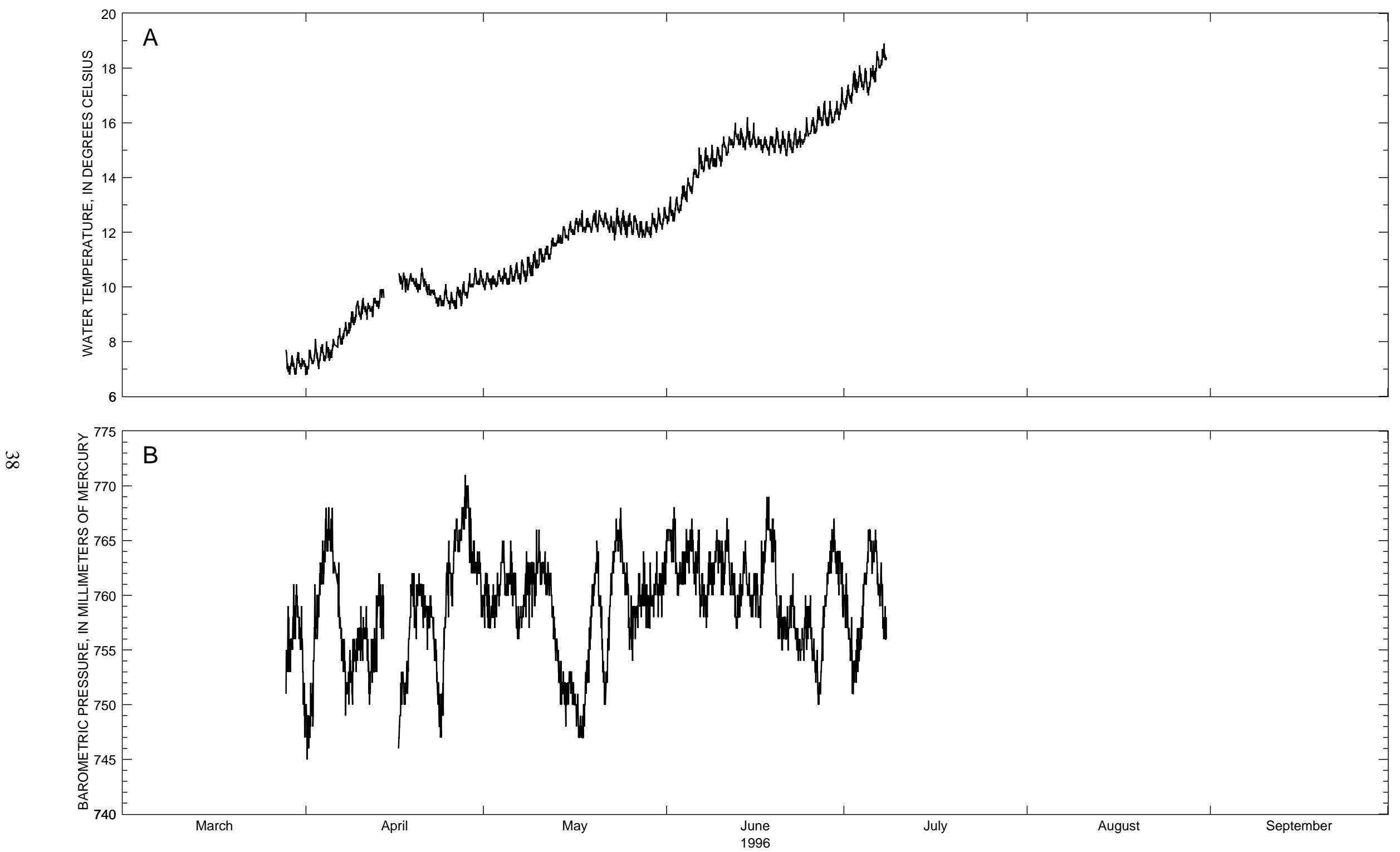

Figure 8. Hourly values of (A) water temperature, (B) barometric pressure, and (C, D) total dissolved gas at the Columbia River, left bank, near The Bales, Oregon, March July 1996. 

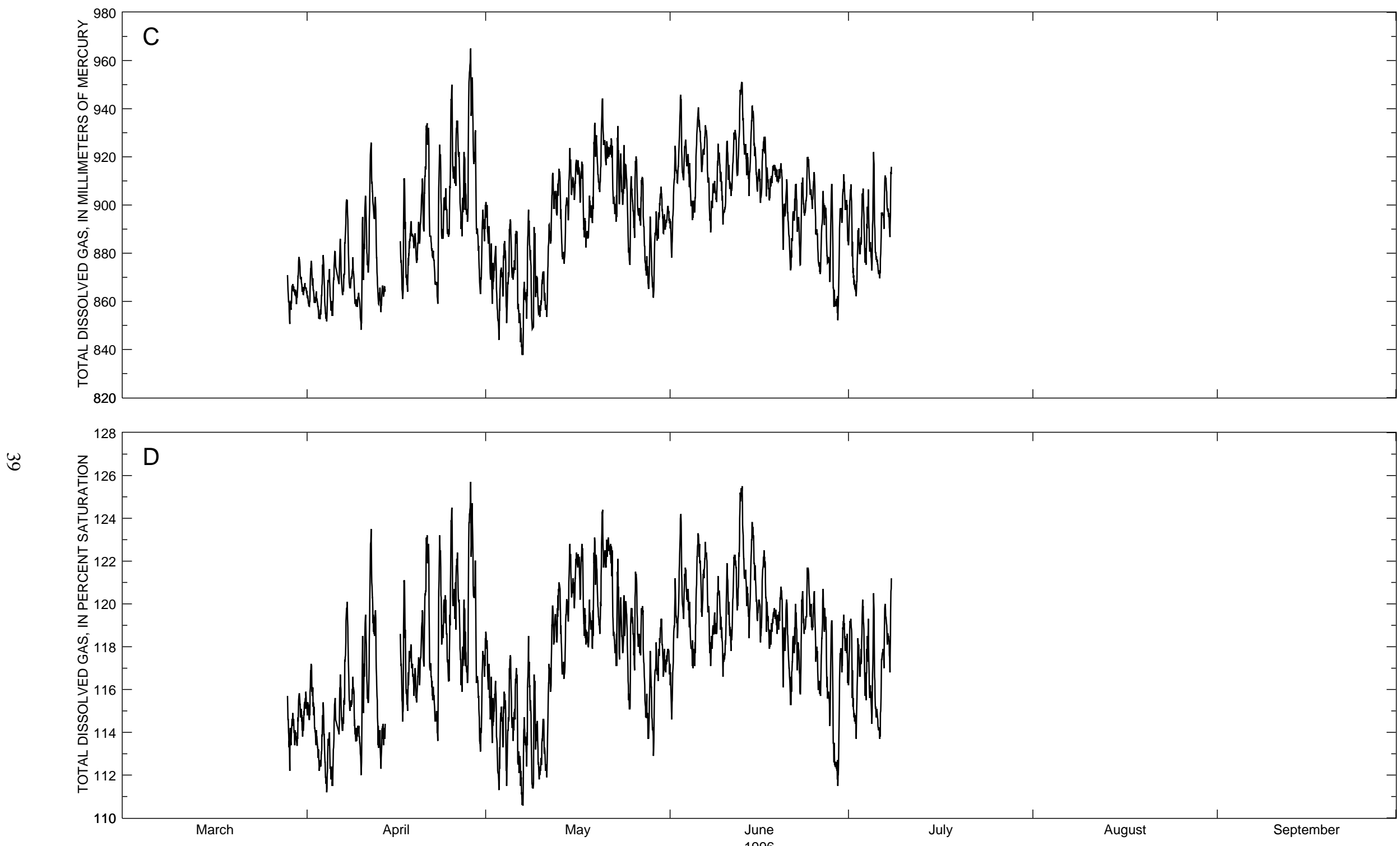

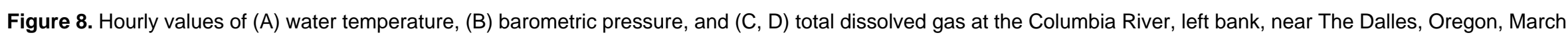
July 1996.—continued 
Table 17. Daily summary of water temperature for the Columbia River, left bank, near The Dalles, Oregon, March - July 1996

[Values reported in degrees Celsius; Max, maximum; Min, minimum; Month, monthly summary statistics; --, not available; e, estimated]

\begin{tabular}{|c|c|c|c|c|c|c|c|c|c|c|c|c|}
\hline \multirow{2}{*}{ Day } & \multicolumn{3}{|c|}{ March } & \multicolumn{3}{|c|}{ April } & \multicolumn{3}{|c|}{ May } & \multicolumn{3}{|c|}{ June } \\
\hline & Max & Min & Mean & Max & Min & Mean & Max & Min & Mean & Max & Min & Mean \\
\hline 1 & & & & 7.7 & 6.8 & 7.2 & 10.5 & 9.9 & 10.2 & 13.3 & 12.3 & 12.7 \\
\hline 2 & & & & 8.1 & 7.2 & 7.5 & 10.4 & 9.9 & 10.2 & 13.3 & 12.4 & 12.8 \\
\hline 3 & & & & 7.9 & 7.0 & 7.5 & 10.6 & 10.0 & 10.3 & 13.7 & 12.7 & 13.1 \\
\hline 4 & & & & 8.0 & 7.3 & 7.6 & 10.7 & 10.1 & 10.3 & 14.0 & 13.1 & 13.5 \\
\hline 5 & & & & 8.1 & 7.4 & 7.7 & 10.8 & 10.1 & 10.4 & 14.3 & 13.4 & 13.9 \\
\hline 6 & & & & 8.5 & -- & -- & 10.9 & 10.2 & 10.5 & 15.1 & 14.0 & 14.4 \\
\hline 7 & & & & 8.7 & 7.9 & 8.3 & 11.0 & 10.1 & 10.5 & 15.1 & 14.2 & 14.6 \\
\hline 8 & & & & e9.1 & e8.3 & 8.6 & 11.1 & 10.2 & 10.6 & 15.2 & 14.3 & 14.6 \\
\hline 9 & & & & e9.5 & 8.6 & e9.1 & 11.3 & 10.4 & 10.8 & 15.1 & 14.4 & 14.7 \\
\hline 10 & & & & 9.6 & 8.8 & 9.2 & 11.4 & 10.7 & 11.0 & 15.5 & 14.4 & 15.0 \\
\hline 11 & & & & 9.4 & 8.8 & 9.2 & 11.5 & 10.9 & 11.2 & 15.5 & 14.8 & 15.1 \\
\hline 12 & & & & 9.6 & 8.9 & 9.3 & 11.8 & 11.0 & 11.4 & 16.0 & 15.1 & 15.4 \\
\hline 13 & & & & 9.9 & 9.2 & 9.6 & 11.9 & 11.5 & 11.7 & 15.8 & 15.1 & 15.4 \\
\hline 14 & & & & -- & -- & -- & 12.2 & 11.6 & 11.9 & 16.2 & 15.0 & 15.4 \\
\hline 15 & & & & -- & -- & -- & 12.4 & 11.7 & 12.0 & 16.0 & 15.1 & 15.4 \\
\hline 16 & & & & -- & -- & -- & 12.5 & 11.9 & 12.2 & 15.5 & 15.0 & 15.3 \\
\hline 17 & & & & 10.5 & 9.8 & 10.2 & 12.8 & 12.0 & 12.3 & 15.5 & 14.9 & 15.2 \\
\hline 18 & & & & 10.5 & 9.9 & 10.2 & 12.5 & 12.0 & 12.2 & 15.5 & 14.8 & 15.2 \\
\hline 19 & & & & 10.3 & 9.8 & 10.1 & 12.7 & 12.0 & 12.3 & 15.8 & 14.9 & 15.3 \\
\hline 20 & & & & 10.7 & 9.9 & 10.2 & 12.8 & 12.0 & 12.4 & 15.7 & 14.9 & 15.2 \\
\hline 21 & & & & 10.3 & 9.7 & 10.0 & 12.7 & 12.2 & 12.4 & 15.7 & 14.8 & 15.2 \\
\hline 22 & & & & 9.9 & 9.6 & 9.8 & 12.6 & 12.0 & 12.2 & 15.8 & 14.9 & 15.3 \\
\hline 23 & & & & 9.7 & 9.3 & 9.5 & 12.9 & 11.7 & 12.3 & 15.7 & 15.1 & 15.3 \\
\hline 24 & & & & 10.1 & 9.3 & 9.6 & 12.8 & 11.9 & 12.3 & 16.2 & 15.2 & 15.6 \\
\hline 25 & & & & 9.8 & 9.2 & 9.5 & 12.7 & 11.8 & 12.3 & 16.2 & 15.6 & 15.8 \\
\hline 26 & & & & 10.0 & 9.2 & 9.6 & 12.6 & 11.9 & 12.3 & 16.6 & 15.6 & 16.1 \\
\hline 27 & & & & 10.2 & 9.3 & 9.7 & 12.4 & 11.8 & 12.1 & 16.8 & 15.9 & 16.2 \\
\hline 28 & & & & e 10.5 & 9.6 & 9.9 & 12.4 & 11.8 & 12.1 & 16.8 & 15.9 & 16.2 \\
\hline 29 & 7.5 & 6.8 & 7.1 & 10.7 & 10.0 & 10.2 & 12.7 & 11.8 & 12.2 & 16.8 & 16.0 & 16.3 \\
\hline 30 & 7.6 & 6.8 & 7.2 & 10.6 & 10.1 & 10.3 & 12.9 & 12.0 & 12.4 & 17.3 & 16.1 & 16.6 \\
\hline 31 & 7.4 & 6.8 & 7.2 & -- & -- & -- & 12.9 & 12.1 & 12.5 & -- & -- & -- \\
\hline Month & -- & -- & -- & -- & -- & -- & 12.9 & 9.9 & 11.6 & 17.3 & 12.3 & 15.0 \\
\hline
\end{tabular}

\begin{tabular}{|c|c|c|c|}
\hline \multirow{2}{*}{ Day } & \multicolumn{3}{|c|}{ July } \\
\hline & Max & Min & Mean \\
\hline 1 & 17.4 & 16.5 & 16.9 \\
\hline 2 & 17.9 & 16.7 & 17.2 \\
\hline 3 & 18.1 & 17.1 & 17.5 \\
\hline 4 & 18.0 & 17.1 & 17.5 \\
\hline 5 & 18.1 & 17.0 & 17.5 \\
\hline 6 & 18.6 & 17.5 & 18.0 \\
\hline 7 & 18.9 & 18.0 & 18.4 \\
\hline 8 & & & \\
\hline 9 & & & \\
\hline 10 & & & \\
\hline 11 & & & \\
\hline 12 & & & \\
\hline 13 & & & \\
\hline 14 & & & \\
\hline 15 & & & \\
\hline 16 & & & \\
\hline 17 & & & \\
\hline 18 & & & \\
\hline 19 & & & \\
\hline 20 & & & \\
\hline 21 & & & \\
\hline 22 & & & \\
\hline 23 & & & \\
\hline 24 & & & \\
\hline 25 & & & \\
\hline 26 & & & \\
\hline 27 & & & \\
\hline 28 & & & \\
\hline 29 & & & \\
\hline 30 & & & \\
\hline 31 & & & \\
\hline Month & & & \\
\hline
\end{tabular}


Table 18. Daily summary of barometric pressure for the Columbia River, left bank, near The Dalles, Oregon, March - July 1996

[Values reported in millimeters of mercury; Max, maximum; Min, minimum; Month, monthly summary statistics; --, not available; e, estimated]

\begin{tabular}{|c|c|c|c|c|c|c|c|c|c|c|c|c|}
\hline \multirow{2}{*}{ Day } & \multicolumn{3}{|c|}{ March } & \multicolumn{3}{|c|}{ April } & \multicolumn{3}{|c|}{ May } & \multicolumn{3}{|c|}{ June } \\
\hline & Max & Min & Mean & Max & Min & Mean & Max & Min & Mean & Max & Min & Mean \\
\hline 1 & & & & 752 & 745 & 748 & 762 & 757 & 760 & 766 & 763 & 765 \\
\hline 2 & & & & 761 & 748 & 755 & 761 & 757 & 758 & 768 & 760 & 764 \\
\hline 3 & & & & 765 & 758 & 761 & 763 & 758 & 760 & 764 & 760 & 762 \\
\hline 4 & & & & 768 & 762 & 765 & 765 & 760 & 762 & 766 & 761 & 764 \\
\hline 5 & & & & 768 & -- & -- & 765 & 759 & 762 & 767 & 760 & 764 \\
\hline 6 & & & & -- & -- & -- & 762 & 756 & 759 & 766 & 758 & 762 \\
\hline 7 & & & & 757 & 749 & 753 & 760 & 756 & 758 & 762 & 757 & 760 \\
\hline 8 & & & & 756 & 750 & 752 & 764 & e757 & 761 & 764 & 760 & 762 \\
\hline 9 & & & & 757 & 753 & 755 & 766 & 758 & 761 & 766 & 761 & 764 \\
\hline 10 & & & & 759 & 754 & 756 & 766 & 761 & 763 & 765 & 760 & 763 \\
\hline 11 & & & & 759 & 750 & 754 & 764 & 760 & 762 & 767 & 760 & 764 \\
\hline 12 & & & & 760 & 752 & 755 & 762 & 756 & 760 & 763 & 757 & 760 \\
\hline 13 & & & & 762 & 756 & 759 & 758 & 752 & 755 & 763 & 758 & 760 \\
\hline 14 & & & & -- & -- & -- & 754 & 748 & 751 & 765 & 760 & 762 \\
\hline 15 & & & & -- & -- & -- & 753 & 750 & 752 & 762 & 758 & 760 \\
\hline 16 & & & & -- & -- & -- & 752 & 748 & 750 & 762 & 757 & 760 \\
\hline 17 & & & & 753 & 749 & 751 & 751 & 747 & 748 & 769 & 760 & 764 \\
\hline 18 & & & & 762 & 751 & 757 & 757 & 748 & 752 & 769 & 763 & 766 \\
\hline 19 & & & & 762 & 756 & 759 & 762 & 755 & 759 & 767 & 756 & 761 \\
\hline 20 & & & & 762 & 757 & 760 & 765 & 757 & 761 & 760 & 755 & 757 \\
\hline 21 & & & & 760 & 755 & 758 & 759 & 750 & 753 & 760 & 755 & 757 \\
\hline 22 & & & & 760 & 754 & 757 & 764 & 753 & 759 & 762 & 756 & 758 \\
\hline 23 & & & & 755 & 747 & 750 & 767 & 762 & 765 & 757 & 754 & 755 \\
\hline 24 & & & & 763 & 749 & 757 & 768 & 760 & 764 & 760 & 754 & 757 \\
\hline 25 & & & & 765 & 758 & 762 & 763 & 755 & 759 & 760 & 754 & 757 \\
\hline 26 & & & & 767 & 764 & 765 & 760 & 754 & 758 & 755 & 750 & 752 \\
\hline 27 & & & & 771 & 765 & 767 & 762 & 757 & 760 & 760 & 751 & 757 \\
\hline 28 & & & & 770 & 762 & 767 & 764 & 757 & 760 & 766 & 760 & 763 \\
\hline 29 & 761 & 753 & 756 & 765 & 761 & 763 & 763 & 757 & 760 & 767 & 762 & 764 \\
\hline 30 & 761 & 756 & 758 & 764 & 758 & 761 & 764 & 758 & 761 & 764 & 758 & 762 \\
\hline 31 & 759 & 747 & 753 & -- & -- & -- & 765 & e758 & 762 & -- & -- & -- \\
\hline Month & -- & -- & -- & -- & -- & -- & 768 & 747 & 759 & 769 & 750 & 761 \\
\hline
\end{tabular}

\begin{tabular}{c|ccc}
\hline \hline \multirow{2}{*}{ Day } & \multicolumn{3}{|c}{ July } \\
\cline { 2 - 4 } & Max & Min & Mean \\
\hline 1 & 762 & 756 & 758 \\
2 & 758 & 751 & 754 \\
3 & 758 & 752 & 755 \\
4 & 763 & 756 & 761 \\
5 & 766 & 762 & 764 \\
6 & 766 & 760 & 763 \\
7 & 763 & 756 & 759 \\
8 & & & \\
9 & & & \\
10 & & & \\
11 & & & \\
12 & & & \\
13 & & & \\
14 & & & \\
15 & & & \\
16 & & & \\
17 & & & \\
18 & & & \\
19 & & & \\
20 & & & \\
21 & & \\
22 & & \\
23 & & \\
24 & & \\
25 & & \\
26 & & \\
27 & & \\
28 & & \\
29 & & \\
30 & & \\
31 & & \\
Month & & \\
\hline
\end{tabular}


Table 19. Daily summary of total dissolved gas, in millimeters of mercury, for the Columbia River, left bank, near The Dalles, Oregon, March - July 1996

[Max, maximum; Min, minimum; Month, monthly summary statistics; --, not available]

\begin{tabular}{|c|c|c|c|c|c|c|c|c|c|c|c|c|}
\hline \multirow{2}{*}{ Day } & \multicolumn{3}{|c|}{ March } & \multicolumn{3}{|c|}{ April } & \multicolumn{3}{|c|}{ May } & \multicolumn{3}{|c|}{ June } \\
\hline & Max & Min & Mean & Max & Min & Mean & Max & Min & Mean & Max & Min & Mean \\
\hline 1 & & & & 877 & 858 & 866 & 901 & 869 & 887 & 925 & 878 & 900 \\
\hline 2 & & & & 865 & 853 & 860 & 883 & 856 & 870 & 946 & 909 & 925 \\
\hline 3 & & & & 879 & 853 & 864 & 879 & 844 & 863 & 927 & 910 & 919 \\
\hline 4 & & & & 873 & 852 & 862 & 890 & 851 & 871 & 921 & 894 & 906 \\
\hline 5 & & & & -- & 854 & -- & 894 & 869 & 879 & 940 & 897 & 922 \\
\hline 6 & & & & -- & -- & -- & 889 & 843 & 865 & 933 & 914 & 923 \\
\hline 7 & & & & 902 & 864 & 885 & 868 & 838 & 854 & 932 & 889 & 909 \\
\hline 8 & & & & 878 & 863 & 869 & 898 & 848 & 873 & 918 & 899 & 906 \\
\hline 9 & & & & 863 & 852 & 859 & 891 & 849 & 868 & 925 & 892 & 911 \\
\hline 10 & & & & 904 & 848 & 880 & 872 & 854 & 863 & 926 & 897 & 910 \\
\hline 11 & & & & 926 & 872 & 897 & 892 & 854 & 873 & 931 & 904 & 917 \\
\hline 12 & & & & 906 & 859 & 887 & 913 & 885 & 903 & 950 & 912 & 929 \\
\hline 13 & & & & 866 & 855 & 862 & 915 & 877 & 899 & 951 & 912 & 929 \\
\hline 14 & & & & -- & -- & -- & 910 & 876 & 891 & 941 & 904 & 924 \\
\hline 15 & & & & -- & -- & -- & 924 & 902 & 911 & 938 & 906 & 917 \\
\hline 16 & & & & -- & -- & -- & 919 & 901 & 913 & 928 & 901 & 916 \\
\hline 17 & & & & 911 & 861 & 880 & 918 & 882 & 899 & 926 & 902 & 910 \\
\hline 18 & & & & 893 & 875 & 886 & 904 & 886 & 894 & 916 & 909 & 914 \\
\hline 19 & & & & 893 & 876 & 885 & 934 & 898 & 919 & 917 & 884 & 910 \\
\hline 20 & & & & 929 & 889 & 903 & 944 & 905 & 923 & 911 & 881 & 897 \\
\hline 21 & & & & 934 & 881 & 908 & 927 & 916 & 922 & 903 & 873 & 887 \\
\hline 22 & & & & 882 & 859 & 871 & 928 & 893 & 908 & 909 & 875 & 893 \\
\hline 23 & & & & 925 & 861 & 900 & 933 & 895 & 913 & 911 & 885 & 901 \\
\hline 24 & & & & 908 & 887 & 896 & 925 & 883 & 907 & 920 & 898 & 910 \\
\hline 25 & & & & 950 & 907 & 922 & 912 & 875 & 894 & 914 & 875 & 895 \\
\hline 26 & & & & 935 & 890 & 915 & 920 & 886 & 904 & 906 & 871 & 887 \\
\hline 27 & & & & 922 & 887 & 903 & 911 & 871 & 894 & 900 & 868 & 883 \\
\hline 28 & & & & 965 & 895 & 942 & 895 & 865 & 877 & 908 & 858 & 876 \\
\hline 29 & 867 & 851 & 862 & 931 & 868 & 899 & 897 & 862 & 881 & 904 & 852 & 880 \\
\hline 30 & 878 & 859 & 869 & 900 & 863 & 884 & 908 & 886 & 898 & 913 & 883 & 900 \\
\hline 31 & 870 & 862 & 865 & -- & -- & -- & 900 & 890 & 894 & -- & -- & -- \\
\hline Month & -- & -- & -- & -- & -- & -- & 944 & 838 & 891 & 951 & 852 & 907 \\
\hline
\end{tabular}

\begin{tabular}{c|ccc}
\hline \hline \multirow{2}{*}{ Day } & \multicolumn{3}{|c}{ July } \\
\cline { 2 - 4 } & Max & Min & Mean \\
\hline 1 & 909 & 867 & 888 \\
2 & 890 & 862 & 875 \\
3 & 907 & 875 & 890 \\
4 & 906 & 873 & 887 \\
5 & 922 & 872 & 889 \\
6 & 899 & 870 & 885 \\
7 & 912 & 887 & 901 \\
8 & & & \\
9 & & & \\
10 & & & \\
11 & & & \\
12 & & & \\
13 & & & \\
14 & & & \\
15 & & & \\
16 & & & \\
17 & & & \\
18 & & & \\
19 & & & \\
20 & & & \\
21 & & \\
22 & & \\
23 & & \\
24 & & \\
25 & & \\
26 & & \\
27 & & \\
28 & & \\
29 & & \\
30 & & \\
31 & & \\
\hline Month & & \\
\hline & & \\
\end{tabular}


Table 20. Daily summary of total dissolved gas, in percent saturation, for the Columbia River, left bank, near The Dalles, Oregon, March - July 1996

[Max, maximum; Min, minimum; Month, monthly summary statistics; --, not available]

\begin{tabular}{|c|c|c|c|c|c|c|c|c|c|c|c|c|}
\hline \multirow{2}{*}{ Day } & \multicolumn{3}{|c|}{ March } & \multicolumn{3}{|c|}{ April } & \multicolumn{3}{|c|}{ May } & \multicolumn{3}{|c|}{ June } \\
\hline & Max & Min & Mean & Max & Min & Mean & Max & Min & Mean & Max & Min & Mean \\
\hline 1 & & & & 117 & 115 & 116 & 119 & 115 & 117 & 121 & 115 & 118 \\
\hline 2 & & & & 115 & 113 & 114 & 116 & 113 & 115 & 124 & 118 & 121 \\
\hline 3 & & & & 115 & 112 & 113 & 115 & 111 & 113 & 122 & 119 & 121 \\
\hline 4 & & & & 114 & 111 & 113 & 117 & 111 & 114 & 121 & 117 & 119 \\
\hline 5 & & & & -- & -- & -- & 118 & 114 & 115 & 123 & 117 & 121 \\
\hline 6 & & & & -- & -- & -- & 117 & 111 & 114 & 123 & 119 & 121 \\
\hline 7 & & & & 120 & 114 & 117 & 115 & 111 & 113 & 122 & 117 & 120 \\
\hline 8 & & & & 117 & 114 & 116 & 118 & 111 & 115 & 120 & 118 & 119 \\
\hline 9 & & & & 115 & 113 & 114 & 117 & 111 & 114 & 121 & 117 & 119 \\
\hline 10 & & & & 119 & 112 & 116 & 115 & 112 & 113 & 122 & 117 & 119 \\
\hline 11 & & & & 123 & 115 & 119 & 117 & 112 & 115 & 122 & 118 & 120 \\
\hline 12 & & & & 120 & 113 & 118 & 120 & 116 & 119 & 125 & 120 & 122 \\
\hline 13 & & & & 114 & 112 & 114 & 121 & 117 & 119 & 125 & 120 & 122 \\
\hline 14 & & & & -- & -- & -- & 121 & 116 & 119 & 124 & 118 & 121 \\
\hline 15 & & & & -- & -- & -- & 123 & 120 & 121 & 123 & 119 & 121 \\
\hline 16 & & & & -- & -- & -- & 122 & 120 & 122 & 122 & 118 & 121 \\
\hline 17 & & & & 121 & 114 & 117 & 123 & 118 & 120 & 122 & 118 & 119 \\
\hline 18 & & & & 118 & 116 & 117 & 120 & 118 & 119 & 120 & 118 & 119 \\
\hline 19 & & & & 117 & 115 & 116 & 123 & 118 & 121 & 121 & 116 & 120 \\
\hline 20 & & & & 122 & 117 & 119 & 124 & 119 & 121 & 120 & 116 & 118 \\
\hline 21 & & & & 123 & 116 & 120 & 123 & 122 & 122 & 119 & 115 & 117 \\
\hline 22 & & & & 116 & 114 & 115 & 123 & 117 & 120 & 120 & 116 & 118 \\
\hline 23 & & & & 123 & 114 & 120 & 122 & 117 & 119 & 121 & 117 & 119 \\
\hline 24 & & & & 120 & 116 & 118 & 120 & 116 & 119 & 122 & 119 & 120 \\
\hline 25 & & & & 124 & 119 & 121 & 120 & 115 & 118 & 121 & 116 & 118 \\
\hline 26 & & & & 122 & 116 & 120 & 121 & 117 & 119 & 121 & 116 & 118 \\
\hline 27 & & & & 120 & 116 & 118 & 120 & 115 & 118 & 120 & 114 & 117 \\
\hline 28 & & & & 126 & 116 & 123 & 118 & 114 & 115 & 119 & 112 & 115 \\
\hline 29 & 115 & 112 & 114 & 122 & 114 & 118 & 118 & 113 & 116 & 118 & 111 & 115 \\
\hline 30 & 116 & 113 & 115 & 118 & 113 & 116 & 119 & 116 & 118 & 119 & 116 & 118 \\
\hline 31 & 116 & 114 & 115 & -- & -- & -- & 118 & 117 & 117 & -- & -- & -- \\
\hline Month & -- & -- & -- & -- & -- & -- & 124 & 111 & 117 & 125 & 111 & 119 \\
\hline \multirow{2}{*}{ Day } & \multicolumn{3}{|c|}{ July } & & & & & & & & & \\
\hline & Max & Min & Mean & & & & & & & & & \\
\hline 1 & 119 & 115 & 117 & & & & & & & & & \\
\hline 2 & 118 & 114 & 116 & & & & & & & & & \\
\hline 3 & 120 & 115 & 118 & & & & & & & & & \\
\hline 4 & 119 & 114 & 117 & & & & & & & & & \\
\hline 5 & 120 & 114 & 116 & & & & & & & & & \\
\hline 6 & 118 & 114 & 116 & & & & & & & & & \\
\hline 7 & 120 & 117 & 119 & & & & & & & & & \\
\hline \multicolumn{13}{|l|}{8} \\
\hline \multicolumn{13}{|l|}{9} \\
\hline 10 & & & & & & & & & & & & \\
\hline 11 & & & & & & & & & & & & \\
\hline 12 & & & & & & & & & & & & \\
\hline 13 & & & & & & & & & & & & \\
\hline 14 & & & & & & & & & & & & \\
\hline 15 & & & & & & & & & & & & \\
\hline 16 & & & & & & & & & & & & \\
\hline 17 & & & & & & & & & & & & \\
\hline 18 & & & & & & & & & & & & \\
\hline 19 & & & & & & & & & & & & \\
\hline 20 & & & & & & & & & & & & \\
\hline 21 & & & & & & & & & & & & \\
\hline 22 & & & & & & & & & & & & \\
\hline 23 & & & & & & & & & & & & \\
\hline 24 & & & & & & & & & & & & \\
\hline 25 & & & & & & & & & & & & \\
\hline 26 & & & & & & & & & & & & \\
\hline 27 & & & & & & & & & & & & \\
\hline 28 & & & & & & & & & & & & \\
\hline 29 & & & & & & & & & & & & \\
\hline 30 & & & & & & & & & & & & \\
\hline 31 & & & & & & & & & & & & \\
\hline Month & & & & & & & & & & & & \\
\hline
\end{tabular}



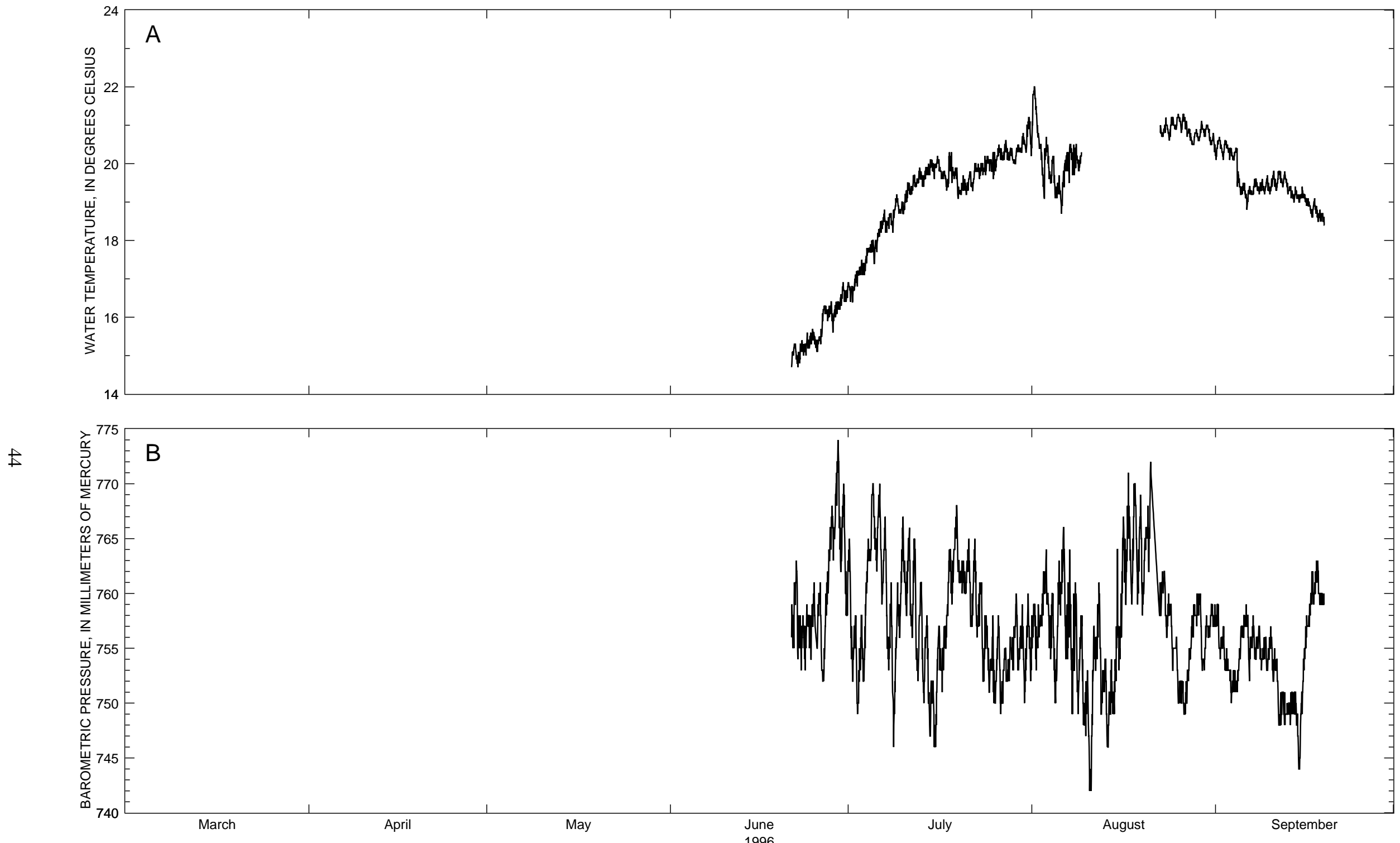

Figure 9. Hourly values of $(A)$ water temperature, $(B)$ barometric pressure, and (C, D) total dissolved gas at the Columbia River at The Dalles, Oregon, June - September 1996. 

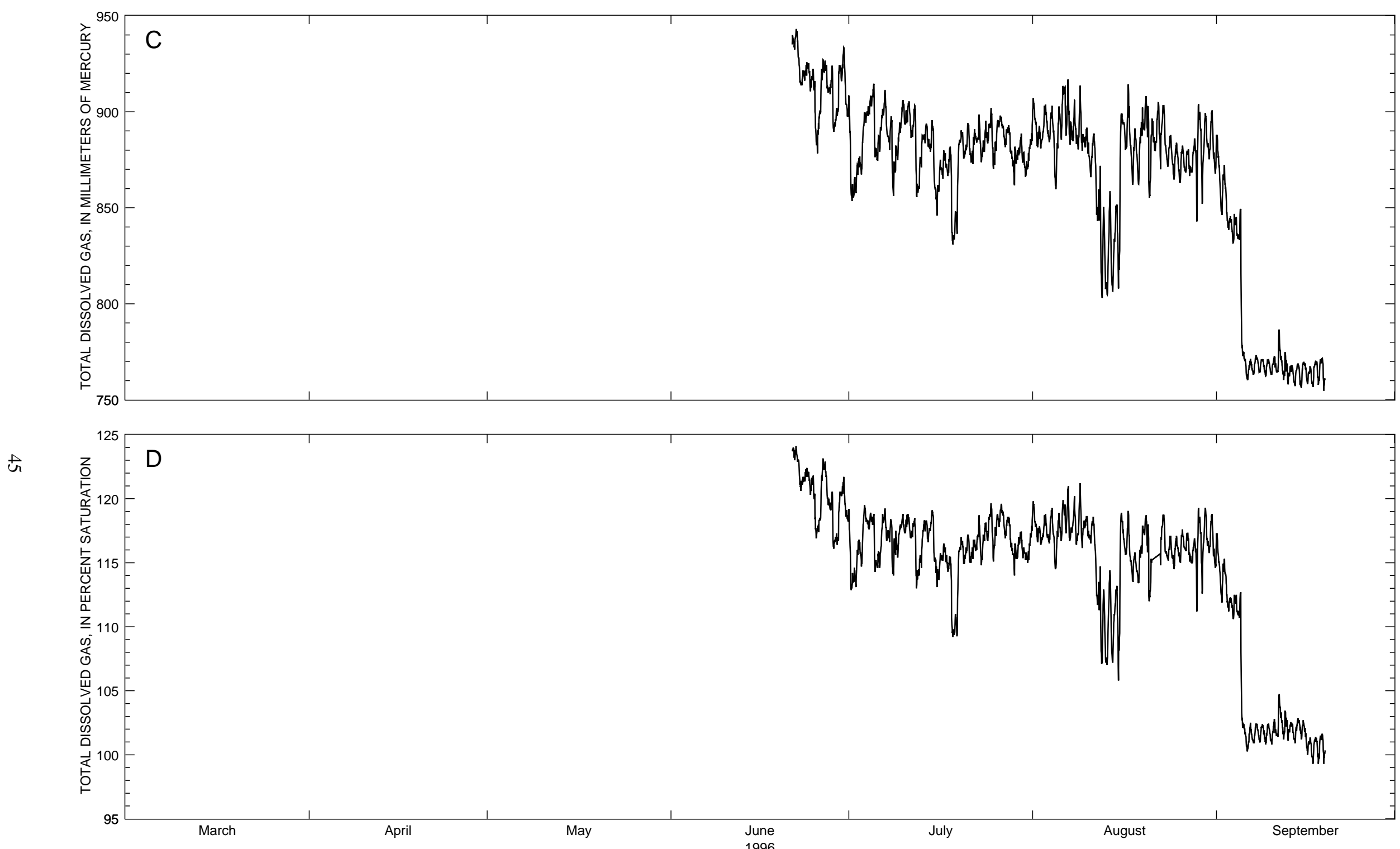

Figure 9. Hourly values of (A) water temperature, (B) barometric pressure, and (C, D) total dissolved gas at the Columbia River at The Dalles, Oregon, June - September 1996.- - continued 
Table 21. Daily summary of water temperature for the Columbia River at The Dalles, Oregon, June - September 1996 [Values reported in degrees Celsius; Max, maximum; Min, minimum; Month, monthly summary statistics; --, not available; e, estimated]

\begin{tabular}{|c|c|c|c|c|c|c|c|c|c|c|c|c|}
\hline \multirow{2}{*}{ Day } & \multicolumn{3}{|c|}{ June } & \multicolumn{3}{|c|}{ July } & \multicolumn{3}{|c|}{ August } & \multicolumn{3}{|c|}{ September } \\
\hline & Max & Min & Mean & Max & Min & Mean & Max & Min & Mean & Max & Min & Mean \\
\hline 1 & -- & -- & -- & 16.9 & 16.4 & 16.7 & 22.0 & 20.6 & 21.5 & 20.7 & 20.1 & 20.5 \\
\hline 2 & -- & -- & -- & 17.3 & 16.7 & 17.1 & 20.8 & 19.4 & 20.2 & 20.6 & 20.1 & 20.4 \\
\hline 3 & -- & -- & -- & 17.5 & 17.1 & 17.3 & 20.7 & 19.1 & 20.1 & 20.5 & 20.1 & 20.3 \\
\hline 4 & -- & -- & -- & 18.0 & 17.4 & 17.7 & 20.2 & 19.1 & 19.7 & 20.4 & 19.4 & 20.0 \\
\hline 5 & -- & -- & -- & 18.2 & 17.4 & 17.8 & 19.7 & 18.7 & 19.3 & 19.6 & 19.2 & 19.3 \\
\hline 6 & -- & -- & -- & 18.6 & 18.1 & 18.3 & 20.3 & 18.7 & 19.7 & 19.4 & 18.8 & 19.2 \\
\hline 7 & -- & -- & -- & 18.8 & 18.2 & 18.5 & 20.5 & 19.5 & 20.2 & 19.6 & 19.2 & 19.4 \\
\hline 8 & -- & -- & -- & 18.9 & 18.2 & 18.6 & 20.5 & 19.7 & 20.1 & 19.6 & 19.2 & 19.4 \\
\hline 9 & -- & -- & -- & 19.2 & 18.7 & 18.9 & -- & -- & -- & 19.7 & 19.2 & 19.4 \\
\hline 10 & -- & -- & -- & 19.3 & 18.7 & 19.0 & -- & -- & -- & 19.8 & 19.2 & 19.5 \\
\hline 11 & -- & -- & -- & 19.7 & 19.1 & 19.4 & -- & -- & -- & 19.8 & 19.3 & 19.6 \\
\hline 12 & -- & -- & -- & 19.8 & 19.4 & 19.6 & -- & -- & -- & 19.8 & 19.4 & 19.6 \\
\hline 13 & -- & -- & -- & 19.9 & 19.4 & 19.6 & -- & -- & -- & 19.5 & 19.1 & 19.3 \\
\hline 14 & -- & -- & -- & 20.1 & 19.6 & 19.9 & -- & -- & -- & 19.4 & 19.0 & 19.2 \\
\hline 15 & -- & -- & -- & 20.1 & 19.6 & 19.9 & -- & -- & -- & 19.4 & 19.0 & 19.2 \\
\hline 16 & -- & -- & -- & 20.2 & 19.6 & 19.9 & -- & -- & -- & 19.2 & 18.8 & 19.0 \\
\hline 17 & -- & -- & -- & 20.2 & 19.3 & 19.6 & -- & -- & -- & 19.1 & 18.6 & 18.8 \\
\hline 18 & -- & -- & -- & e20.3 & 19.5 & 19.9 & -- & -- & -- & 18.9 & 18.5 & 18.7 \\
\hline 19 & -- & -- & -- & e19.9 & 19.1 & 19.5 & -- & -- & -- & & & \\
\hline 20 & -- & -- & -- & 19.7 & 19.2 & 19.4 & -- & -- & -- & & & \\
\hline 21 & -- & -- & -- & 19.8 & 19.3 & 19.5 & -- & -- & -- & & & \\
\hline 22 & 15.3 & 14.7 & 15.0 & 20.0 & 19.3 & 19.8 & -- & -- & -- & & & \\
\hline 23 & 15.4 & 15.0 & 15.2 & 20.0 & 19.6 & 19.8 & 21.2 & 20.7 & 20.9 & & & \\
\hline 24 & 15.7 & 15.2 & 15.4 & 20.2 & 19.7 & 20.0 & 21.2 & 20.6 & 21.0 & & & \\
\hline 25 & 15.7 & 15.1 & 15.4 & 20.3 & 19.6 & 20.0 & 21.3 & 20.9 & 21.1 & & & \\
\hline 26 & 16.3 & 15.3 & 15.7 & 20.5 & 20.1 & 20.3 & 21.3 & 20.8 & 21.1 & & & \\
\hline 27 & 16.3 & 15.9 & 16.1 & 20.6 & 20.1 & 20.3 & 21.1 & 20.5 & 20.8 & & & \\
\hline 28 & 16.4 & 15.6 & 16.1 & 20.4 & 20.0 & 20.2 & 20.9 & 20.5 & 20.7 & & & \\
\hline 29 & 16.6 & 16.1 & 16.4 & 20.5 & 20.0 & 20.3 & 21.1 & 20.6 & 20.8 & & & \\
\hline 30 & 16.9 & 16.4 & 16.7 & 20.8 & 20.3 & 20.5 & 21.0 & 20.6 & 20.9 & & & \\
\hline 31 & -- & -- & -- & 21.2 & 20.2 & 20.8 & 20.8 & 20.2 & 20.6 & & & \\
\hline Month & -- & -- & -- & 21.2 & 16.4 & 19.3 & -- & -- & -- & & & \\
\hline
\end{tabular}


Table 22. Daily summary of barometric pressure for the Columbia River at The Dalles, Oregon, June - September 1996

[Values reported in millimeters of mercury; Max, maximum; Min, minimum; Month, monthly summary statistics; --, not available; e, estimated]

\begin{tabular}{|c|c|c|c|c|c|c|c|c|c|c|c|c|}
\hline \multirow{2}{*}{ Day } & \multicolumn{3}{|c|}{ June } & \multicolumn{3}{|c|}{ July } & \multicolumn{3}{|c|}{ August } & \multicolumn{3}{|c|}{ September } \\
\hline & Max & Min & Mean & Max & Min & Mean & Max & Min & Mean & Max & Min & Mean \\
\hline 1 & -- & -- & -- & 765 & 752 & 758 & 759 & 753 & 757 & 759 & 754 & 757 \\
\hline 2 & -- & -- & -- & 758 & 749 & 753 & 762 & 755 & 758 & 757 & 753 & 755 \\
\hline 3 & -- & -- & -- & 760 & 752 & 755 & 764 & 755 & 760 & 754 & 750 & 752 \\
\hline 4 & -- & -- & -- & 769 & 760 & 763 & 760 & 750 & 756 & 754 & 751 & 752 \\
\hline 5 & -- & -- & -- & 770 & 762 & 766 & 763 & 750 & 758 & 758 & 754 & 756 \\
\hline 6 & -- & -- & -- & 770 & 759 & 764 & 766 & 754 & 760 & 759 & 752 & 756 \\
\hline 7 & -- & -- & -- & 767 & 753 & 760 & 764 & 749 & 756 & 758 & 754 & 756 \\
\hline 8 & -- & -- & -- & 761 & 746 & 754 & 761 & 749 & 755 & 757 & 753 & 755 \\
\hline 9 & -- & -- & -- & 762 & 754 & 758 & 758 & 748 & 753 & 756 & 753 & 754 \\
\hline 10 & -- & -- & -- & 767 & 758 & 762 & 753 & 742 & 748 & 757 & 752 & 754 \\
\hline 11 & -- & -- & -- & 766 & 756 & 761 & 757 & 742 & 753 & 754 & 748 & 751 \\
\hline 12 & -- & -- & -- & 765 & 752 & 758 & 761 & 749 & 755 & 751 & 748 & 750 \\
\hline 13 & -- & -- & -- & 761 & 750 & 756 & 754 & 746 & 750 & 751 & 748 & 750 \\
\hline 14 & -- & -- & -- & 758 & 747 & 753 & 754 & 749 & 751 & 751 & 745 & 749 \\
\hline 15 & -- & -- & -- & 753 & 746 & 749 & 764 & 751 & 756 & 755 & 744 & 749 \\
\hline 16 & -- & -- & -- & 757 & 751 & 754 & 767 & 756 & 762 & 759 & 754 & 757 \\
\hline 17 & -- & -- & -- & 763 & 753 & 757 & 771 & 759 & 764 & 762 & 759 & 760 \\
\hline 18 & -- & -- & -- & 764 & 759 & 762 & 770 & 759 & 765 & 763 & 759 & 761 \\
\hline 19 & -- & -- & -- & 768 & 761 & 764 & 769 & 758 & 763 & & & \\
\hline 20 & -- & -- & -- & 763 & 760 & 761 & 771 & 762 & 765 & & & \\
\hline 21 & -- & -- & -- & 765 & 757 & 761 & -- & -- & -- & & & \\
\hline 22 & 763 & 754 & 758 & 765 & 756 & 760 & -- & -- & -- & & & \\
\hline 23 & 758 & 753 & 756 & 761 & 752 & 757 & 762 & 756 & 759 & & & \\
\hline 24 & 759 & 754 & 757 & 758 & 751 & 755 & 760 & 753 & 757 & & & \\
\hline 25 & e761 & -- & -- & 758 & 750 & 753 & 756 & 750 & 753 & & & \\
\hline 26 & 761 & 752 & 756 & 758 & 749 & 753 & 752 & 749 & 750 & & & \\
\hline 27 & 766 & 754 & 760 & 755 & 750 & 753 & 757 & 750 & 753 & & & \\
\hline 28 & 770 & 763 & 766 & 757 & 752 & 754 & 760 & 756 & 758 & & & \\
\hline 29 & 774 & 762 & 768 & 760 & 753 & 756 & 760 & 753 & 757 & & & \\
\hline 30 & 770 & 758 & 764 & 759 & 750 & 755 & 758 & 753 & 756 & & & \\
\hline 31 & -- & -- & -- & 760 & 752 & 756 & 759 & 756 & 758 & & & \\
\hline Month & -- & -- & -- & 770 & 746 & 757 & -- & -- & -- & & & \\
\hline
\end{tabular}


Table 23. Daily summary of total dissolved gas, in millimeters of mercury, for the Columbia River at The Dalles, Oregon, June - September 1996

[Max, maximum; Min, minimum; Month, monthly summary statistics; --, not available]

\begin{tabular}{|c|c|c|c|c|c|c|c|c|c|c|c|c|}
\hline \multirow{2}{*}{ Day } & \multicolumn{3}{|c|}{ June } & \multicolumn{3}{|c|}{ July } & \multicolumn{3}{|c|}{ August } & \multicolumn{3}{|c|}{ September } \\
\hline & Max & Min & Mean & Max & Min & Mean & Max & Min & Mean & Max & Min & Mean \\
\hline 1 & -- & -- & -- & 908 & 853 & 869 & 907 & 881 & 894 & 888 & 846 & 867 \\
\hline 2 & -- & -- & -- & 877 & 858 & 869 & 903 & 881 & 889 & 872 & 839 & 857 \\
\hline 3 & -- & -- & -- & 900 & 867 & 888 & 904 & 884 & 893 & 847 & 832 & 840 \\
\hline 4 & -- & -- & -- & 908 & 898 & 903 & 903 & 860 & 884 & 849 & 833 & 839 \\
\hline 5 & -- & -- & -- & 915 & 875 & 889 & 904 & 870 & 891 & 849 & 764 & 784 \\
\hline 6 & -- & -- & -- & 905 & 879 & 893 & 917 & 888 & 905 & 771 & 760 & 766 \\
\hline 7 & -- & -- & -- & 911 & 880 & 893 & 913 & 883 & 892 & 773 & 763 & 768 \\
\hline 8 & -- & -- & -- & 898 & 856 & 876 & 914 & 880 & 892 & 771 & 764 & 768 \\
\hline 9 & -- & -- & -- & 901 & 875 & 888 & 914 & 880 & 888 & 771 & 762 & 767 \\
\hline 10 & -- & -- & -- & 906 & 893 & 900 & 892 & 866 & 878 & 773 & 763 & 768 \\
\hline 11 & -- & -- & -- & 905 & 886 & 895 & 889 & 843 & 871 & 787 & 764 & 772 \\
\hline 12 & -- & -- & -- & 903 & 856 & 873 & 872 & 803 & 838 & 774 & 760 & 767 \\
\hline 13 & -- & -- & -- & 893 & 871 & 883 & 858 & 805 & 823 & 768 & 758 & 765 \\
\hline 14 & -- & -- & -- & 894 & 879 & 886 & 859 & 806 & 828 & 769 & 757 & 764 \\
\hline 15 & -- & -- & -- & 896 & 846 & 869 & 899 & 808 & 850 & 769 & 756 & 764 \\
\hline 16 & -- & -- & -- & 880 & 858 & 868 & 899 & 880 & 890 & 767 & 758 & 763 \\
\hline 17 & -- & -- & -- & 881 & 866 & 873 & 914 & 862 & 885 & 770 & 757 & 765 \\
\hline 18 & -- & -- & -- & 882 & 831 & 849 & 891 & 862 & 877 & 771 & 758 & 766 \\
\hline 19 & -- & -- & -- & 890 & 836 & 869 & 904 & 878 & 891 & -- & -- & -- \\
\hline 20 & -- & -- & -- & 890 & 876 & 882 & 908 & 855 & 885 & -- & -- & -- \\
\hline 21 & -- & -- & -- & 894 & 873 & 882 & 896 & 879 & 887 & -- & -- & -- \\
\hline 22 & 943 & 914 & 929 & 898 & 883 & 888 & 905 & 870 & 893 & -- & -- & -- \\
\hline 23 & 926 & 914 & 920 & 895 & 874 & 882 & 903 & 871 & 884 & -- & -- & -- \\
\hline 24 & 925 & 911 & 919 & 902 & 883 & 890 & 887 & 865 & 877 & -- & -- & -- \\
\hline 25 & 922 & 878 & 896 & 902 & 870 & 883 & 884 & 863 & 874 & -- & -- & -- \\
\hline 26 & 927 & 897 & 915 & 898 & 891 & 895 & 882 & 868 & 875 & -- & -- & -- \\
\hline 27 & 927 & 909 & 915 & 893 & 882 & 888 & 880 & 866 & 873 & -- & -- & -- \\
\hline 28 & 924 & 890 & 902 & 891 & 862 & 877 & 904 & 843 & 879 & -- & -- & -- \\
\hline 29 & 928 & 898 & 916 & 886 & 873 & 879 & 900 & 852 & 882 & -- & -- & -- \\
\hline 30 & 933 & 897 & 912 & 889 & 866 & 875 & 899 & 875 & 886 & -- & -- & -- \\
\hline 31 & -- & -- & -- & 901 & 870 & 881 & 901 & 868 & 883 & -- & -- & -- \\
\hline Month & -- & -- & -- & 915 & 831 & 882 & 917 & 803 & 879 & -- & -- & -- \\
\hline
\end{tabular}


Table 24. Daily summary of total dissolved gas, in percent saturation, for the Columbia River at The Dalles, Oregon, June - September 1996

[Max, maximum; Min, minimum; Month, monthly summary statistics; --, not available]

\begin{tabular}{|c|c|c|c|c|c|c|c|c|c|c|c|c|}
\hline \multirow{2}{*}{ Day } & \multicolumn{3}{|c|}{ June } & \multicolumn{3}{|c|}{ July } & \multicolumn{3}{|c|}{ August } & \multicolumn{3}{|c|}{ September } \\
\hline & Max & Min & Mean & Max & Min & Mean & Max & Min & Mean & Max & Min & Mean \\
\hline 1 & & & & 119 & 113 & 115 & 120 & 117 & 118 & 117 & 112 & 115 \\
\hline 2 & & & & 117 & 113 & 115 & 119 & 116 & 117 & 115 & 111 & 114 \\
\hline 3 & & & & 119 & 115 & 118 & 119 & 116 & 117 & 112 & 111 & 112 \\
\hline 4 & & & & 119 & 118 & 118 & 119 & 114 & 117 & 113 & 111 & 112 \\
\hline 5 & & & & 119 & 114 & 116 & 119 & 116 & 118 & 113 & 101 & 104 \\
\hline 6 & & & & 119 & 115 & 117 & 121 & 117 & 119 & 102 & 100 & 101 \\
\hline 7 & & & & 119 & 117 & 118 & 121 & 117 & 118 & 102 & 101 & 102 \\
\hline 8 & & & & 118 & 114 & 116 & 121 & 116 & 118 & 102 & 101 & 102 \\
\hline 9 & & & & 118 & 115 & 117 & 121 & 116 & 118 & 102 & 101 & 102 \\
\hline 10 & & & & 119 & 117 & 118 & 119 & 116 & 118 & 103 & 101 & 102 \\
\hline 11 & & & & 119 & 117 & 118 & 119 & 112 & 116 & 105 & 101 & 103 \\
\hline 12 & & & & 118 & 113 & 115 & 115 & 107 & 111 & -- & -- & -- \\
\hline 13 & & & & 118 & 115 & 117 & 114 & 107 & 110 & 102 & 101 & 102 \\
\hline 14 & & & & 119 & 117 & 118 & 114 & 107 & 110 & 103 & 101 & 102 \\
\hline 15 & & & & 119 & 113 & 116 & 119 & 106 & 112 & 103 & 101 & 102 \\
\hline 16 & & & & 116 & 114 & 115 & 119 & 116 & 117 & 102 & 100 & 101 \\
\hline 17 & & & & 116 & 114 & 115 & 119 & 113 & 116 & 101 & 99 & 101 \\
\hline 18 & & & & 115 & 109 & 111 & 116 & 113 & 115 & 102 & 99 & 101 \\
\hline 19 & & & & 117 & 109 & 114 & 118 & 115 & 117 & & & \\
\hline 20 & & & & 117 & 115 & 116 & 119 & 112 & 116 & & & \\
\hline 21 & & & & 117 & 115 & 116 & -- & -- & -- & & & \\
\hline 22 & 124 & 121 & 123 & 119 & 116 & 117 & -- & -- & -- & & & \\
\hline 23 & 122 & 121 & 122 & 118 & 115 & 116 & 119 & 115 & 116 & & & \\
\hline 24 & 122 & 120 & 121 & 120 & 117 & 118 & 117 & 114 & 116 & & & \\
\hline 25 & 122 & -- & -- & 120 & 115 & 117 & 117 & 115 & 116 & & & \\
\hline 26 & 123 & 118 & 121 & 120 & 118 & 119 & 118 & 116 & 117 & & & \\
\hline 27 & 123 & 119 & 120 & 119 & 117 & 118 & 117 & 115 & 116 & & & \\
\hline 28 & 120 & 116 & 118 & 118 & 114 & 116 & 119 & 111 & 116 & & & \\
\hline 29 & 121 & 116 & 119 & 117 & 115 & 116 & 119 & 113 & 117 & & & \\
\hline 30 & 122 & 118 & 119 & 117 & 115 & 116 & 119 & 116 & 117 & & & \\
\hline 31 & -- & -- & -- & 119 & 115 & 116 & 119 & 115 & 117 & & & \\
\hline Month & -- & -- & -- & 120 & 109 & 116 & -- & -- & -- & & & \\
\hline
\end{tabular}



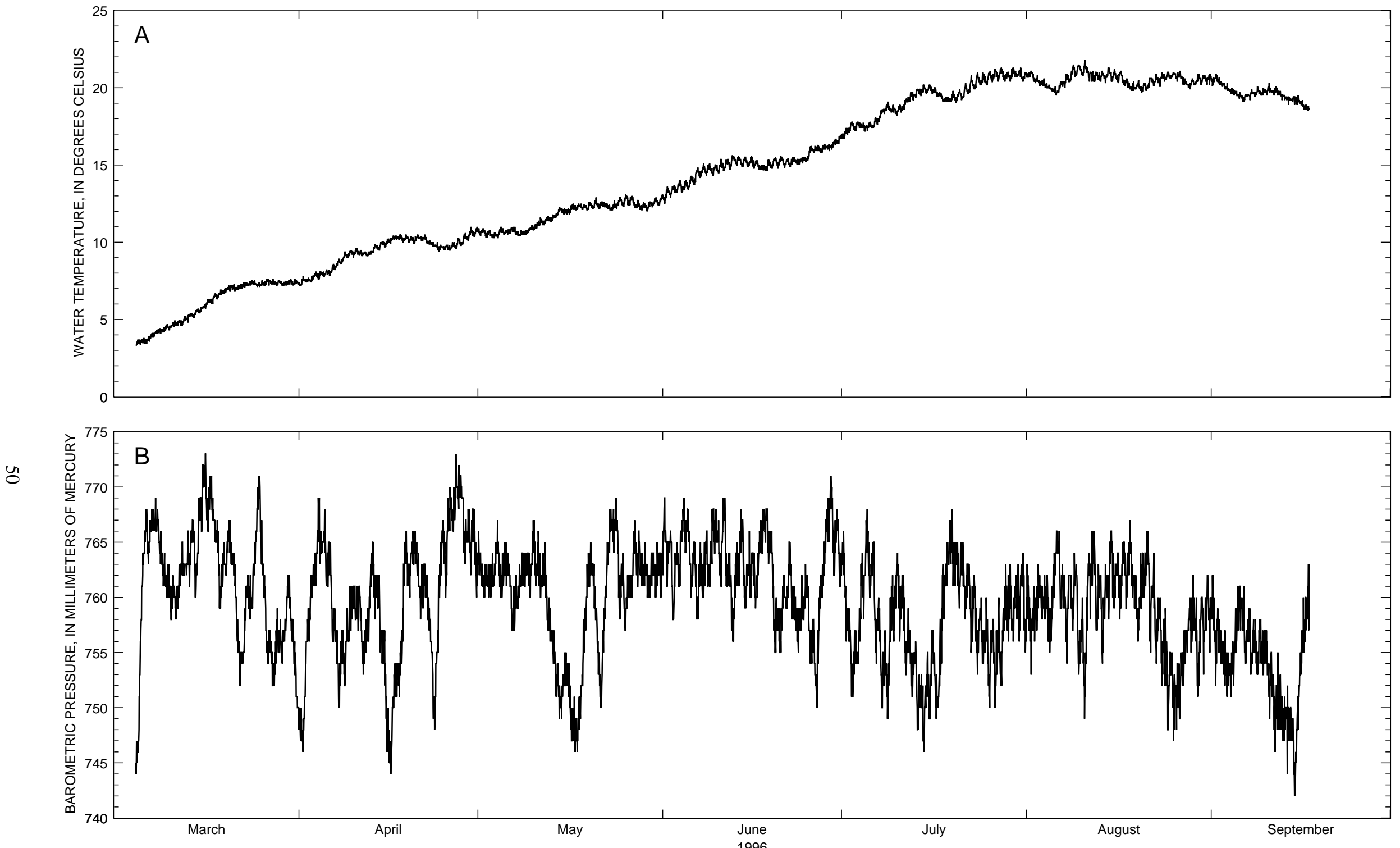

Figure 10. Hourly values of (A) water temperature, (B) barometric pressure, and (C, D) total dissolved gas at the Columbia River at Bonneville Dam forebay, Washington, March - September 1996. 


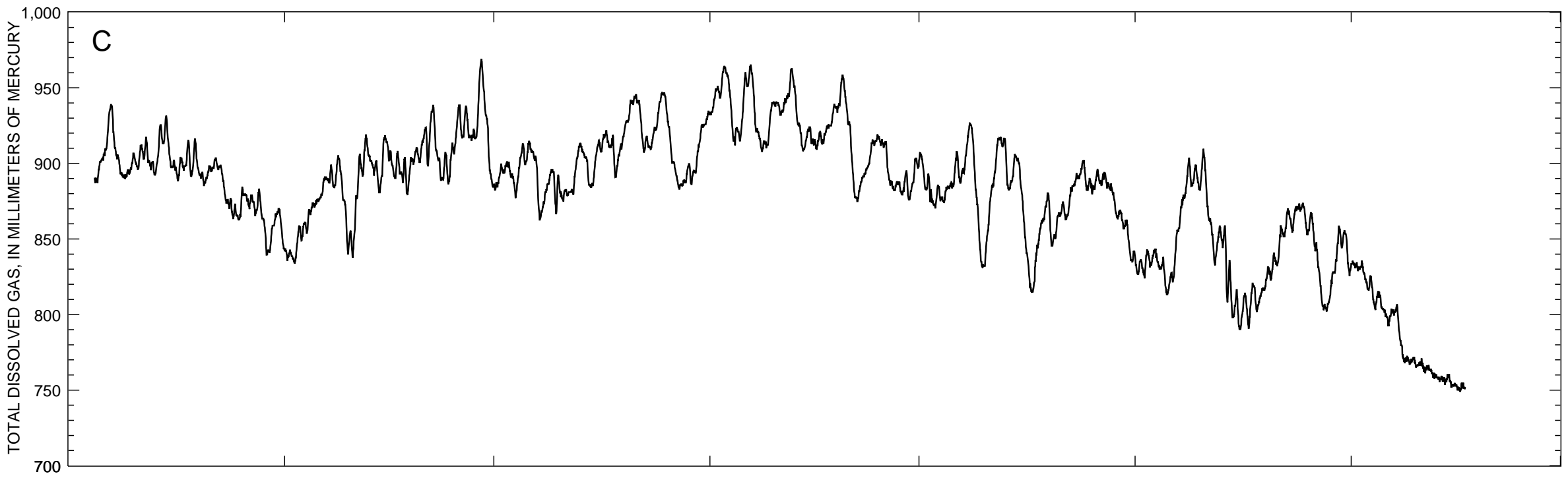

ur

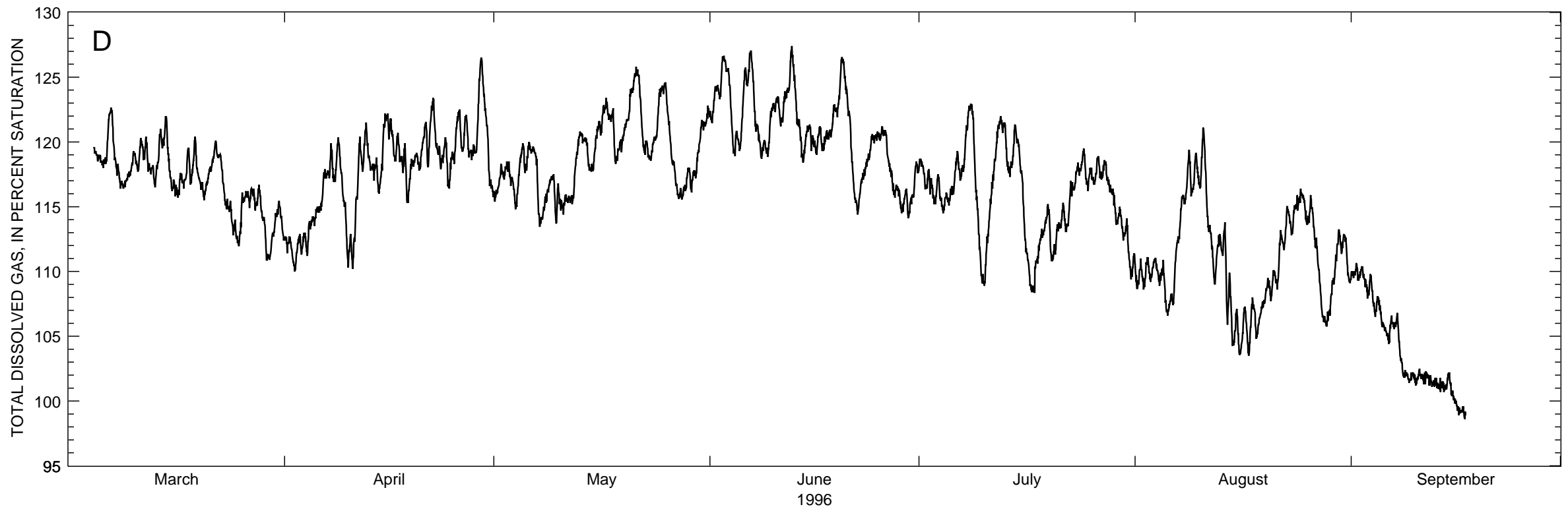

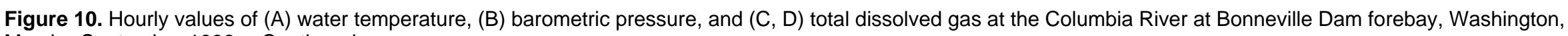
March - September 1996. -Continued 
Table 25. Daily summary of water temperature for the Columbia River at Bonneville Dam forebay, Washington, March - September 1996

[Values reported in degrees Celsius; Max, maximum; Min, minimum; Month, monthly summary statistics; --, not available; e, estimated]

\begin{tabular}{|c|c|c|c|c|c|c|c|c|c|c|c|c|}
\hline \multirow{2}{*}{ Day } & \multicolumn{3}{|c|}{ March } & \multicolumn{3}{|c|}{ April } & \multicolumn{3}{|c|}{ May } & \multicolumn{3}{|c|}{ June } \\
\hline & Max & Min & Mean & Max & Min & Mean & Max & Min & Mean & Max & Min & Mean \\
\hline 1 & -- & -- & -- & 7.8 & 7.2 & 7.4 & 10.9 & 10.4 & 10.7 & 13.6 & 12.5 & 13.0 \\
\hline 2 & -- & -- & -- & 7.7 & 7.4 & 7.5 & 10.8 & 10.3 & 10.6 & 13.7 & 12.9 & 13.3 \\
\hline 3 & -- & -- & -- & 8.1 & 7.4 & 7.8 & 10.8 & 10.3 & 10.5 & 14.0 & 13.2 & 13.6 \\
\hline 4 & -- & -- & -- & 8.1 & 7.6 & 7.9 & 11.0 & 10.3 & 10.6 & 14.0 & 13.2 & 13.6 \\
\hline 5 & 3.8 & 3.4 & 3.6 & 8.2 & 7.8 & 8.0 & 10.9 & 10.5 & 10.7 & 14.3 & 13.4 & 13.8 \\
\hline 6 & 3.9 & 3.4 & 3.6 & 8.6 & 7.8 & 8.2 & 11.0 & 10.5 & 10.8 & 14.8 & 13.7 & 14.3 \\
\hline 7 & 4.2 & 3.8 & 4.0 & 8.9 & 8.2 & 8.6 & 11.0 & 10.4 & 10.6 & 15.1 & 14.2 & 14.6 \\
\hline 8 & 4.4 & 4.1 & 4.2 & 9.4 & 8.6 & 9.0 & 10.8 & 10.4 & 10.6 & 15.1 & 14.3 & 14.7 \\
\hline 9 & 4.7 & 4.2 & 4.4 & 9.5 & 9.0 & 9.3 & 10.9 & 10.5 & 10.8 & 15.0 & 14.3 & 14.7 \\
\hline 10 & 4.8 & 4.3 & 4.5 & 9.6 & 9.0 & 9.4 & 11.3 & 10.8 & 11.0 & 15.4 & 14.5 & 14.9 \\
\hline 11 & 4.9 & 4.5 & 4.8 & 9.5 & 9.1 & 9.3 & 11.6 & 11.1 & 11.3 & 15.4 & 14.6 & 15.0 \\
\hline 12 & 5.2 & 4.6 & 4.9 & 9.4 & 9.1 & 9.3 & 11.7 & 11.1 & 11.4 & 15.6 & 14.7 & 15.2 \\
\hline 13 & 5.4 & 4.8 & 5.2 & 9.9 & 9.2 & 9.5 & 11.8 & 11.3 & 11.5 & 15.6 & 15.0 & 15.3 \\
\hline 14 & 5.6 & 5.1 & 5.4 & 10.0 & 9.5 & 9.7 & 12.3 & 11.6 & 11.9 & 15.5 & 14.9 & 15.2 \\
\hline 15 & 5.9 & 5.4 & 5.6 & 10.2 & 9.7 & 9.9 & 12.2 & 11.8 & 12.0 & 15.6 & 14.9 & 15.2 \\
\hline 16 & 6.3 & 5.7 & 6.0 & 10.4 & 9.9 & 10.2 & 12.5 & 11.8 & 12.1 & 15.5 & 14.8 & 15.1 \\
\hline 17 & 6.7 & 6.0 & 6.3 & 10.5 & 10.2 & 10.3 & 12.5 & 12.1 & 12.3 & 15.0 & 14.7 & 14.8 \\
\hline 18 & 6.9 & 6.3 & 6.6 & 10.5 & 10.0 & 10.2 & 12.5 & 12.1 & 12.3 & e 15.3 & 14.6 & e14.9 \\
\hline 19 & 7.0 & 6.7 & 6.8 & 10.4 & 10.0 & 10.2 & 12.6 & 12.1 & 12.3 & 15.5 & 14.8 & 15.1 \\
\hline 20 & 7.2 & 6.8 & 7.0 & 10.5 & 9.9 & 10.2 & 12.9 & 12.2 & 12.4 & 15.6 & 14.8 & 15.2 \\
\hline 21 & 7.3 & 6.8 & 7.0 & 10.4 & 10.1 & 10.3 & 12.6 & 12.2 & 12.4 & 15.4 & 14.8 & 15.2 \\
\hline 22 & 7.4 & 7.0 & 7.2 & 10.5 & 9.9 & 10.1 & 12.6 & 12.2 & 12.3 & 15.5 & 14.9 & 15.2 \\
\hline 23 & 7.5 & 7.2 & 7.3 & 10.1 & 9.7 & 9.8 & 12.7 & 12.1 & 12.3 & 15.5 & 15.0 & 15.2 \\
\hline 24 & 7.5 & 7.2 & 7.4 & 10.0 & 9.4 & 9.7 & 12.9 & 12.2 & 12.5 & 15.5 & 15.0 & 15.3 \\
\hline 25 & 7.5 & 7.1 & 7.3 & 9.8 & 9.5 & 9.7 & 13.1 & 12.3 & 12.7 & 16.2 & 15.2 & 15.7 \\
\hline 26 & 7.6 & 7.2 & 7.4 & 10.0 & 9.5 & 9.7 & 13.0 & 12.4 & 12.7 & 16.2 & 15.8 & 16.0 \\
\hline 27 & 7.6 & 7.3 & 7.4 & 10.3 & 9.6 & 9.9 & 12.9 & 12.2 & 12.5 & 16.3 & 15.8 & 16.1 \\
\hline 28 & 7.5 & 7.2 & 7.4 & 10.6 & 9.8 & 10.1 & 12.6 & 12.1 & 12.3 & 16.3 & 15.9 & 16.2 \\
\hline 29 & 7.5 & 7.2 & 7.3 & 11.0 & 10.1 & 10.5 & 12.6 & 12.0 & 12.3 & 16.6 & 16.0 & 16.3 \\
\hline 30 & 7.6 & 7.3 & 7.4 & 11.0 & 10.4 & 10.6 & 12.9 & 12.3 & 12.6 & 17.0 & 16.4 & 16.6 \\
\hline 31 & 7.6 & 7.2 & 7.4 & -- & -- & -- & 13.1 & 12.4 & 12.7 & -- & -- & -- \\
\hline Month & -- & -- & -- & 11.0 & 7.2 & 9.4 & 13.1 & 10.3 & 11.7 & 17.0 & 12.5 & 15.0 \\
\hline \multirow{2}{*}{ Day } & \multicolumn{3}{|c|}{ July } & \multicolumn{3}{|c|}{ August } & \multicolumn{3}{|c|}{ September } & & & \\
\hline & Max & Min & Mean & Max & Min & Mean & Max & Min & Mean & & & \\
\hline 1 & 17.4 & 16.7 & 17.0 & 21.1 & 20.7 & 20.9 & 20.9 & 20.1 & 20.5 & & & \\
\hline 2 & 17.8 & 17.0 & 17.5 & 20.9 & 20.3 & 20.5 & e 20.7 & 20.1 & 20.3 & & & \\
\hline 3 & 17.8 & 17.2 & 17.5 & e20.6 & 20.1 & 20.3 & 20.3 & 19.8 & 20.0 & & & \\
\hline 4 & 17.7 & 17.2 & 17.5 & 20.3 & 19.9 & 20.1 & $\mathrm{e} 20.0$ & 19.5 & 19.8 & & & \\
\hline 5 & 17.8 & 17.2 & 17.5 & 20.1 & 19.5 & 19.9 & 19.8 & 19.3 & 19.6 & & & \\
\hline 6 & 18.1 & 17.4 & 17.7 & 20.4 & 19.5 & 20.0 & 19.6 & 19.1 & 19.4 & & & \\
\hline 7 & 18.6 & 17.6 & 18.2 & 20.9 & 20.0 & 20.4 & 19.9 & 19.2 & 19.6 & & & \\
\hline 8 & 19.1 & 18.3 & 18.7 & 21.5 & 20.4 & 20.8 & 20.1 & 19.4 & 19.7 & & & \\
\hline 9 & 18.9 & 18.4 & 18.6 & 21.5 & 20.7 & 21.1 & 20.0 & 19.5 & 19.7 & & & \\
\hline 10 & 18.9 & 18.2 & 18.5 & e21.8 & 20.8 & 21.2 & 20.3 & 19.5 & 19.9 & & & \\
\hline 11 & 19.3 & 18.5 & 18.9 & 21.4 & 20.4 & 20.9 & 20.1 & 19.5 & 19.8 & & & \\
\hline 12 & 19.7 & 19.1 & 19.4 & 21.1 & 20.4 & 20.7 & 20.0 & 19.2 & 19.6 & & & \\
\hline 13 & 19.9 & 19.2 & 19.6 & 21.1 & 20.4 & 20.7 & 19.5 & e18.9 & 19.4 & & & \\
\hline 14 & 20.2 & 19.5 & 19.8 & 21.3 & 20.3 & 20.8 & 19.4 & e18.9 & 19.2 & & & \\
\hline 15 & 20.2 & 19.6 & 19.9 & 21.2 & 20.4 & 20.8 & 19.5 & e18.9 & 19.2 & & & \\
\hline 16 & 20.0 & 19.4 & 19.8 & 21.1 & 20.3 & 20.7 & 19.2 & 18.6 & 18.8 & & & \\
\hline 17 & 19.7 & 19.1 & 19.5 & 20.8 & 20.1 & 20.4 & & & & & & \\
\hline 18 & 19.4 & 19.1 & 19.2 & 20.4 & 19.8 & 20.1 & & & & & & \\
\hline 19 & 19.8 & 19.1 & 19.3 & 20.5 & 19.9 & 20.1 & & & & & & \\
\hline 20 & 19.9 & 19.0 & 19.5 & 20.3 & 19.7 & 20.0 & & & & & & \\
\hline 21 & 20.3 & 19.2 & 19.7 & 20.6 & 19.8 & 20.2 & & & & & & \\
\hline 22 & 20.8 & 19.6 & 20.1 & 20.9 & 20.1 & 20.5 & & & & & & \\
\hline 23 & 21.0 & 19.9 & 20.3 & 21.0 & 20.1 & 20.6 & & & & & & \\
\hline 24 & 21.0 & 20.2 & 20.5 & 21.0 & 20.4 & 20.7 & & & & & & \\
\hline 25 & 21.1 & 20.2 & 20.6 & 21.0 & 20.6 & 20.8 & & & & & & \\
\hline 26 & 21.2 & 20.4 & 20.8 & 21.1 & 20.4 & 20.7 & & & & & & \\
\hline 27 & 21.3 & 20.4 & 20.9 & 20.7 & 20.2 & 20.4 & & & & & & \\
\hline 28 & 21.1 & 20.4 & 20.8 & 20.6 & 19.9 & 20.2 & & & & & & \\
\hline 29 & 21.3 & 20.4 & 20.9 & 20.9 & 20.2 & 20.4 & & & & & & \\
\hline 30 & 21.1 & 20.7 & 20.9 & 20.8 & 20.2 & 20.6 & & & & & & \\
\hline 31 & 21.3 & 20.4 & 20.7 & 20.9 & 20.2 & 20.6 & & & & & & \\
\hline Month & 21.3 & 16.7 & 19.3 & 21.8 & 19.5 & 20.5 & & & & & & \\
\hline
\end{tabular}


Table 26. Daily summary of barometric pressure for the Columbia River at Bonneville Dam forebay, Washington, March - September 1996

[Values reported in millimeters of mercury; Max, maximum; Min, minimum; Month, monthly summary statistics; --, not available; e, estimated]

\begin{tabular}{|c|c|c|c|c|c|c|c|c|c|c|c|c|}
\hline \multirow{2}{*}{ Day } & \multicolumn{3}{|c|}{ March } & \multicolumn{3}{|c|}{ April } & \multicolumn{3}{|c|}{ May } & \multicolumn{3}{|c|}{ June } \\
\hline & Max & Min & Mean & Max & Min & Mean & Max & Min & Mean & Max & Min & Mean \\
\hline 1 & & & & 751 & 746 & 748 & 766 & 761 & 763 & 769 & 761 & 765 \\
\hline 2 & & & & 762 & 751 & 757 & 763 & 760 & 762 & 766 & 758 & 762 \\
\hline 3 & & & & 764 & 760 & 762 & 764 & 760 & 762 & 766 & 761 & 763 \\
\hline 4 & & & & 769 & 763 & 765 & 767 & 760 & 763 & 769 & 764 & 766 \\
\hline 5 & 764 & 746 & 756 & 768 & 761 & 764 & 765 & 760 & 763 & 768 & 760 & 764 \\
\hline 6 & 768 & 763 & 765 & 765 & 756 & 760 & 764 & 757 & 760 & 765 & 758 & 762 \\
\hline 7 & 769 & 765 & 767 & 759 & 750 & 754 & 763 & 757 & 760 & 765 & 760 & 762 \\
\hline 8 & 768 & 763 & 766 & 757 & 752 & 755 & 764 & 761 & 762 & 766 & 761 & 763 \\
\hline 9 & 764 & 760 & 762 & 761 & 756 & 758 & 764 & 760 & 763 & 768 & 763 & 766 \\
\hline 10 & 763 & 758 & 760 & 761 & 757 & 759 & 767 & 761 & 764 & 768 & 761 & 764 \\
\hline 11 & 763 & 758 & 760 & 761 & 753 & 757 & 766 & 760 & 762 & 769 & 761 & 765 \\
\hline 12 & 765 & 761 & 763 & 762 & 754 & 758 & 765 & 757 & 760 & 764 & 756 & 760 \\
\hline 13 & 766 & 761 & 764 & 765 & 758 & 762 & 760 & 754 & 756 & 766 & 758 & 762 \\
\hline 14 & 767 & 760 & 764 & 762 & 754 & 759 & 754 & 749 & 752 & 768 & 759 & 763 \\
\hline 15 & 772 & 764 & 768 & 757 & 746 & 752 & 755 & 749 & 752 & 766 & 760 & 762 \\
\hline 16 & 773 & 766 & 769 & 753 & 744 & 748 & 754 & 747 & 751 & 764 & 759 & 762 \\
\hline 17 & 771 & 765 & 768 & 755 & 751 & 753 & 751 & 746 & 748 & 768 & 762 & 765 \\
\hline 18 & 767 & 759 & 763 & 766 & 754 & 760 & 759 & 748 & 753 & 768 & 763 & 766 \\
\hline 19 & 765 & 761 & 763 & 766 & 760 & 763 & 765 & 757 & 761 & 766 & 755 & 761 \\
\hline 20 & 767 & 763 & 765 & 766 & 762 & 764 & 764 & 757 & 761 & 760 & 755 & 757 \\
\hline 21 & 764 & 754 & 759 & 764 & 758 & 761 & 757 & 750 & 754 & 762 & 758 & 760 \\
\hline 22 & 759 & 752 & 755 & 763 & 757 & 760 & 765 & 755 & 761 & 765 & 758 & 762 \\
\hline 23 & 762 & 758 & 760 & 758 & 748 & 753 & 768 & 764 & 766 & 761 & 755 & 758 \\
\hline 24 & 768 & 759 & 763 & 766 & 754 & 760 & 769 & 758 & 764 & 763 & 757 & 759 \\
\hline 25 & 771 & 762 & 767 & 767 & 760 & 764 & 764 & 757 & 760 & 762 & 754 & 758 \\
\hline 26 & 763 & 754 & 758 & 770 & 766 & 768 & 762 & 759 & 761 & 758 & 750 & 754 \\
\hline 27 & 757 & 752 & 754 & 773 & 767 & 770 & 767 & 761 & 764 & 763 & 754 & 760 \\
\hline 28 & 759 & 753 & 756 & 771 & 763 & 768 & 766 & 761 & 763 & 769 & 762 & 766 \\
\hline 29 & 760 & 754 & 757 & 768 & 762 & 765 & 765 & 760 & 761 & 771 & 762 & 767 \\
\hline 30 & 762 & 756 & 759 & 768 & 760 & 765 & 764 & 760 & 762 & 768 & 761 & 764 \\
\hline 31 & 758 & 748 & 753 & -- & -- & -- & 766 & 760 & 763 & -- & -- & -- \\
\hline Month & -- & -- & -- & 773 & 744 & 760 & 769 & 746 & 760 & 771 & 750 & 762 \\
\hline \multirow{2}{*}{ Day } & \multicolumn{3}{|c|}{ July } & \multicolumn{3}{|c|}{$\overline{\text { August }}$} & \multicolumn{3}{|c|}{ September } & & & \\
\hline & Max & Min & Mean & Max & Min & Mean & Max & Min & Mean & & & \\
\hline 1 & 766 & 756 & 761 & 763 & 753 & 759 & 762 & e752 & 758 & & & \\
\hline 2 & 759 & 751 & 755 & 763 & 756 & 759 & 759 & 754 & 756 & & & \\
\hline 3 & 759 & 753 & 755 & 763 & 758 & 760 & 757 & 751 & 754 & & & \\
\hline 4 & 764 & 756 & 761 & 762 & 755 & 759 & 756 & 751 & 754 & & & \\
\hline 5 & 768 & 760 & 764 & 764 & 756 & 761 & 761 & 755 & 759 & & & \\
\hline 6 & 765 & 754 & 760 & 766 & 759 & 763 & 761 & 754 & 757 & & & \\
\hline 7 & 760 & 750 & 755 & 763 & 754 & 759 & 759 & 753 & 756 & & & \\
\hline 8 & 757 & 749 & 753 & 762 & 757 & 760 & 758 & 753 & 755 & & & \\
\hline 9 & 762 & 756 & 758 & 764 & 753 & 759 & 758 & 753 & 756 & & & \\
\hline 10 & 764 & 756 & 760 & 760 & e749 & 755 & 757 & 749 & 754 & & & \\
\hline 11 & 762 & 753 & 758 & 766 & 754 & 761 & 755 & 746 & 751 & & & \\
\hline 12 & 759 & 751 & 755 & 766 & 757 & 762 & 755 & 748 & 751 & & & \\
\hline 13 & 756 & 748 & 752 & 763 & 754 & 759 & 752 & 744 & 749 & & & \\
\hline 14 & 753 & 746 & 750 & 765 & 758 & 761 & 750 & 742 & 748 & & & \\
\hline 15 & 755 & 749 & 752 & 766 & 759 & 763 & 755 & 742 & 749 & & & \\
\hline 16 & 757 & 749 & 754 & 764 & 758 & 762 & 760 & 753 & 757 & & & \\
\hline 17 & 763 & 750 & 755 & 765 & 759 & 763 & & & & & & \\
\hline 18 & 765 & 758 & 762 & 767 & 760 & 763 & & & & & & \\
\hline 19 & 768 & 761 & 764 & 762 & 756 & 759 & & & & & & \\
\hline 20 & 765 & 759 & 763 & 764 & 759 & 762 & & & & & & \\
\hline 21 & 765 & 758 & 762 & 766 & e755 & 762 & & & & & & \\
\hline 22 & 763 & 755 & 760 & 764 & 754 & 759 & & & & & & \\
\hline 23 & 762 & 753 & 758 & 760 & 752 & 757 & & & & & & \\
\hline 24 & 760 & 754 & 757 & 759 & 748 & 754 & & & & & & \\
\hline 25 & 760 & 750 & 755 & 755 & 747 & 752 & & & & & & \\
\hline 26 & 758 & 750 & 755 & 755 & 748 & 752 & & & & & & \\
\hline 27 & 760 & 752 & 755 & 757 & 752 & 755 & & & & & & \\
\hline 28 & 763 & 756 & 759 & 762 & 755 & 758 & & & & & & \\
\hline 29 & 763 & 756 & 759 & 760 & 751 & 756 & & & & & & \\
\hline 30 & 763 & 756 & 760 & 760 & 753 & 758 & & & & & & \\
\hline 31 & 764 & 755 & 760 & 762 & 755 & 759 & & & & & & \\
\hline Month & 768 & 746 & 758 & 767 & 747 & 759 & & & & & & \\
\hline
\end{tabular}


Table 27. Daily summary of total dissolved gas, in millimeters of mercury, for the Columbia River at Bonneville Dam forebay, Washington, March - September 1996

[Max, maximum; Min, minimum; Month, monthly summary statistics; --, not available; e, estimated]

\begin{tabular}{|c|c|c|c|c|c|c|c|c|c|c|c|c|}
\hline \multirow{2}{*}{ Day } & \multicolumn{3}{|c|}{ March } & \multicolumn{3}{|c|}{ April } & \multicolumn{3}{|c|}{ May } & \multicolumn{3}{|c|}{ June } \\
\hline & Max & Min & Mean & Max & Min & Mean & Max & Min & Mean & Max & Min & Mean \\
\hline 1 & -- & -- & -- & 843 & 835 & 840 & 897 & 882 & 888 & 949 & 932 & 940 \\
\hline 2 & -- & -- & -- & 855 & 834 & 841 & 901 & 894 & 897 & 963 & 943 & 951 \\
\hline 3 & -- & -- & -- & 861 & 849 & 856 & 901 & 884 & 893 & 965 & 940 & 956 \\
\hline 4 & -- & -- & -- & 874 & 854 & 864 & 907 & 877 & 892 & 940 & 912 & 922 \\
\hline 5 & 904 & 887 & 897 & 877 & 871 & 874 & 913 & 899 & 906 & 956 & 915 & 928 \\
\hline 6 & 936 & 902 & 917 & 891 & 876 & 884 & 914 & 902 & 908 & 965 & 951 & 957 \\
\hline 7 & 939 & 903 & 921 & 899 & 885 & 891 & 905 & 863 & 877 & 960 & 919 & 935 \\
\hline 8 & 906 & 891 & 896 & 905 & 884 & 895 & 889 & 871 & 881 & 919 & 908 & 913 \\
\hline 9 & 898 & 890 & 893 & 895 & 847 & 875 & 896 & 866 & 888 & 940 & 910 & 924 \\
\hline 10 & 907 & 896 & 901 & 856 & 838 & 847 & 892 & 868 & 881 & 941 & 935 & 939 \\
\hline 11 & 913 & 897 & 906 & 906 & 853 & 885 & 883 & 875 & 881 & 943 & 932 & 937 \\
\hline 12 & 917 & 895 & 905 & 919 & 891 & 906 & 904 & 879 & 889 & 963 & 943 & 952 \\
\hline 13 & 915 & 892 & 899 & 906 & 892 & 900 & 913 & 904 & 909 & 955 & 920 & 935 \\
\hline 14 & 931 & 913 & 920 & 902 & 881 & 890 & 906 & 884 & 895 & 921 & 908 & 913 \\
\hline 15 & 931 & 897 & 909 & 919 & 892 & 911 & 912 & 884 & 898 & 924 & 912 & 918 \\
\hline 16 & 902 & 888 & 895 & 908 & 890 & 898 & 919 & 907 & 914 & 921 & 909 & 915 \\
\hline 17 & 906 & 895 & 900 & 908 & 887 & 897 & 922 & 911 & 915 & 926 & 913 & 919 \\
\hline 18 & 915 & 891 & 902 & 904 & 879 & 891 & 919 & 891 & 902 & 939 & 923 & 930 \\
\hline 19 & 916 & 890 & 902 & 910 & 897 & 904 & 927 & 905 & 916 & 958 & 934 & 942 \\
\hline 20 & 894 & e885 & 890 & 918 & 900 & 908 & 942 & 927 & 934 & 958 & 926 & 942 \\
\hline 21 & 902 & 894 & 897 & 930 & 899 & 915 & 946 & 934 & 942 & 928 & 877 & 897 \\
\hline 22 & 903 & 894 & 899 & 939 & 903 & 922 & 934 & 907 & 917 & 891 & 875 & 883 \\
\hline 23 & 894 & 873 & 881 & 905 & 889 & 895 & 922 & 909 & 913 & 905 & 891 & 896 \\
\hline 24 & 877 & 863 & 870 & 910 & 886 & 898 & 944 & 922 & 931 & 916 & 905 & 913 \\
\hline 25 & 884 & 862 & 869 & 938 & 906 & 918 & 947 & 927 & 942 & 919 & 911 & 915 \\
\hline 26 & 883 & 870 & 877 & 938 & 917 & 926 & 928 & 896 & 909 & 915 & 885 & 900 \\
\hline 27 & 879 & 865 & 873 & 938 & 915 & 922 & 896 & 883 & 888 & 888 & 882 & 885 \\
\hline 28 & 883 & 862 & 872 & 960 & 916 & 927 & 900 & 885 & 891 & 890 & 879 & 884 \\
\hline 29 & 862 & 839 & 847 & 969 & 929 & 951 & 900 & 886 & 893 & 895 & 876 & 884 \\
\hline 30 & 868 & 847 & 860 & 929 & 884 & 900 & 926 & 894 & 913 & 903 & 885 & 896 \\
\hline 31 & 870 & 842 & 858 & -- & -- & -- & 935 & 924 & 929 & -- & -- & -- \\
\hline Month & -- & -- & -- & 969 & 834 & 894 & 947 & 863 & 904 & 965 & 875 & 921 \\
\hline \multirow{2}{*}{ Day } & \multicolumn{3}{|c|}{ July } & \multicolumn{3}{|c|}{ August } & \multicolumn{3}{|c|}{ September } & & & \\
\hline & Max & Min & Mean & Max & Min & Mean & Max & Min & Mean & & & \\
\hline 1 & 907 & 882 & 897 & 838 & 826 & 832 & 836 & 829 & 833 & & & \\
\hline 2 & 893 & 874 & 883 & 843 & 824 & 834 & 836 & 823 & 830 & & & \\
\hline 3 & 886 & 870 & 878 & 844 & 831 & 838 & 826 & 816 & 821 & & & \\
\hline 4 & 884 & 874 & 878 & 840 & 830 & 833 & 818 & 803 & 810 & & & \\
\hline 5 & 888 & 883 & 885 & 838 & 813 & 821 & 815 & 798 & 805 & & & \\
\hline 6 & 908 & 886 & 897 & 854 & 822 & 832 & 803 & 792 & 799 & & & \\
\hline 7 & 918 & 888 & 900 & 879 & 854 & 866 & 806 & 784 & 799 & & & \\
\hline 8 & 927 & 894 & 918 & 904 & 876 & 890 & 784 & 768 & 774 & & & \\
\hline 9 & 894 & 833 & 862 & 899 & 885 & 891 & 773 & 767 & 770 & & & \\
\hline 10 & 859 & 831 & 841 & 910 & 882 & 894 & 772 & 765 & 767 & & & \\
\hline 11 & 898 & 859 & 883 & 900 & 855 & 871 & 771 & 761 & 765 & & & \\
\hline 12 & 917 & 898 & 913 & 855 & 832 & 844 & 766 & 757 & 762 & & & \\
\hline 13 & 917 & 882 & 900 & 859 & 843 & 853 & 760 & 755 & 758 & & & \\
\hline 14 & 906 & 883 & 895 & 843 & 798 & 818 & 761 & 753 & 757 & & & \\
\hline 15 & 904 & 865 & 890 & 816 & 790 & 803 & 761 & 752 & 754 & & & \\
\hline 16 & 865 & 818 & 840 & 814 & 790 & 804 & 755 & 749 & 752 & & & \\
\hline 17 & 846 & 814 & 826 & 821 & 790 & 806 & & & & & & \\
\hline 18 & 866 & 844 & 857 & 819 & 802 & 809 & & & & & & \\
\hline 19 & 881 & 845 & 869 & 829 & 812 & 819 & & & & & & \\
\hline 20 & 865 & 845 & 854 & 841 & 823 & 830 & & & & & & \\
\hline 21 & 875 & 863 & 869 & 859 & 832 & 842 & & & & & & \\
\hline 22 & 885 & 863 & 872 & 870 & 851 & 859 & & & & & & \\
\hline 23 & 894 & 884 & 889 & 870 & 854 & 863 & & & & & & \\
\hline 24 & 902 & 885 & 895 & 873 & 868 & 871 & & & & & & \\
\hline 25 & 890 & 880 & 885 & 873 & 853 & 862 & & & & & & \\
\hline 26 & 896 & 883 & 889 & 868 & 842 & 856 & & & & & & \\
\hline 27 & 894 & 883 & 890 & 848 & 805 & 823 & & & & & & \\
\hline 28 & 887 & 876 & 883 & 813 & 802 & 806 & & & & & & \\
\hline 29 & 876 & 863 & 868 & 845 & 813 & 828 & & & & & & \\
\hline 30 & 867 & 852 & 860 & 858 & 844 & 851 & & & & & & \\
\hline 31 & 852 & 835 & 840 & 855 & 825 & 840 & & & & & & \\
\hline Month & 927 & 814 & 878 & 910 & 790 & 842 & & & & & & \\
\hline
\end{tabular}


Table 28. Daily summary of total dissolved gas, in percent saturation, for the Columbia River at Bonneville Dam forebay, Washington, March - September 1996

[Max, maximum; Min, minimum; Month, monthly summary statistics; --, not available; e, estimated]

\begin{tabular}{|c|c|c|c|c|c|c|c|c|c|c|c|c|}
\hline \multirow{2}{*}{ Day } & \multicolumn{3}{|c|}{ March } & \multicolumn{3}{|c|}{ April } & \multicolumn{3}{|c|}{ May } & \multicolumn{3}{|c|}{ June } \\
\hline & Max & Min & Mean & Max & Min & Mean & Max & Min & Mean & Max & Min & Mean \\
\hline 1 & -- & -- & -- & 113 & 111 & 112 & 118 & 115 & 116 & 124 & 121 & 123 \\
\hline 2 & -- & -- & -- & 112 & 110 & 111 & 118 & 117 & 118 & 127 & 123 & 125 \\
\hline 3 & -- & -- & -- & 113 & 111 & 112 & 118 & 116 & 117 & 127 & 123 & 125 \\
\hline 4 & -- & -- & -- & 114 & 111 & 113 & 119 & 115 & 117 & 123 & 119 & 120 \\
\hline 5 & 119 & 118 & 119 & 115 & 113 & 114 & 120 & 118 & 119 & 125 & 119 & 121 \\
\hline 6 & 122 & 118 & 120 & 118 & 115 & 116 & 120 & 119 & 119 & 127 & 124 & 126 \\
\hline 7 & 123 & 117 & 120 & 120 & 117 & 118 & 119 & 113 & 115 & 126 & 120 & 123 \\
\hline 8 & 118 & 116 & 117 & 120 & 117 & 119 & 117 & 114 & 116 & 121 & 119 & 120 \\
\hline 9 & 118 & 116 & 117 & 118 & 111 & 115 & 117 & 114 & 116 & 123 & 119 & 121 \\
\hline 10 & 119 & 118 & 119 & 113 & 110 & 112 & 117 & 114 & 115 & 123 & 122 & 123 \\
\hline 11 & 120 & 118 & 119 & 120 & 112 & 117 & 116 & 114 & 116 & 124 & 121 & 122 \\
\hline 12 & 120 & 117 & 119 & 121 & 118 & 120 & 119 & 115 & 117 & 127 & 123 & 125 \\
\hline 13 & 120 & 116 & 118 & 119 & 117 & 118 & 121 & 119 & 120 & 126 & 120 & 123 \\
\hline 14 & 122 & 119 & 120 & 119 & 116 & 117 & 120 & 118 & 119 & 121 & 118 & 120 \\
\hline 15 & 122 & 116 & 118 & 122 & 118 & 121 & 121 & 118 & 119 & 121 & 119 & 121 \\
\hline 16 & 117 & 116 & 116 & 122 & 118 & 120 & 123 & 120 & 122 & 121 & 119 & 120 \\
\hline 17 & 118 & 116 & 117 & 121 & 118 & 119 & 123 & 122 & 122 & 121 & 119 & 120 \\
\hline 18 & 119 & 117 & 118 & 120 & 115 & 117 & 123 & 118 & 120 & 123 & 120 & 121 \\
\hline 19 & 120 & 116 & 118 & 119 & 117 & 118 & 121 & 119 & 120 & 126 & 122 & 124 \\
\hline 20 & 117 & e115 & 116 & 120 & 118 & 119 & 124 & 121 & 123 & 126 & 122 & 124 \\
\hline 21 & 120 & 117 & 118 & 122 & 118 & 120 & 126 & 123 & 125 & 122 & 115 & 118 \\
\hline 22 & 120 & 118 & 119 & 123 & 119 & 121 & 123 & 119 & 121 & 118 & 114 & 116 \\
\hline 23 & 118 & 115 & 116 & 120 & 118 & 119 & 120 & 119 & 119 & 119 & 117 & 118 \\
\hline 24 & 115 & 113 & 114 & 120 & 116 & 118 & 124 & 120 & 122 & 121 & 119 & 120 \\
\hline 25 & 116 & 112 & 113 & 122 & 118 & 120 & 125 & 122 & 124 & 121 & 120 & 121 \\
\hline 26 & 116 & 115 & 116 & 122 & 119 & 121 & 122 & 118 & 119 & 121 & 117 & 119 \\
\hline 27 & 116 & 115 & 116 & 122 & 119 & 120 & 118 & 116 & 116 & 118 & 116 & 117 \\
\hline 28 & 117 & 114 & 115 & 125 & 119 & 121 & 118 & 116 & 117 & 116 & 114 & 115 \\
\hline 29 & 114 & 111 & 112 & 126 & 121 & 124 & 118 & 116 & 117 & 116 & 114 & 115 \\
\hline 30 & 115 & 111 & 113 & 121 & 116 & 118 & 122 & 117 & 120 & 118 & 116 & 117 \\
\hline 31 & 115 & 112 & 114 & -- & -- & -- & 123 & 121 & 122 & -- & -- & -- \\
\hline Month & -- & -- & -- & 126 & 110 & 118 & 126 & 113 & 119 & 127 & 114 & 121 \\
\hline \multirow{2}{*}{ Day } & \multicolumn{3}{|c|}{ July } & \multicolumn{3}{|c|}{ August } & \multicolumn{3}{|c|}{ September } & & & \\
\hline & Max & Min & Mean & Max & Min & Mean & Max & Min & Mean & & & \\
\hline 1 & 119 & 116 & 118 & 111 & 109 & 110 & 111 & 109 & 110 & & & \\
\hline 2 & 118 & 116 & 117 & 111 & 109 & 110 & 110 & 109 & 110 & & & \\
\hline 3 & 117 & 115 & 116 & 111 & 109 & 110 & 110 & 108 & 109 & & & \\
\hline 4 & 116 & 114 & 115 & 111 & 109 & 110 & 108 & 106 & 107 & & & \\
\hline 5 & 117 & 115 & 116 & 111 & 107 & 108 & 108 & 105 & 106 & & & \\
\hline 6 & 119 & 116 & 118 & 112 & 107 & 109 & 107 & 104 & 105 & & & \\
\hline 7 & 122 & 117 & 119 & 116 & 112 & 114 & 107 & 103 & 106 & & & \\
\hline 8 & 123 & 118 & 122 & 119 & 115 & 117 & 103 & 102 & 102 & & & \\
\hline 9 & 118 & 110 & 114 & 119 & 116 & 117 & 102 & 101 & 102 & & & \\
\hline 10 & 113 & 109 & 111 & 121 & 116 & 118 & 102 & 101 & 102 & & & \\
\hline 11 & 119 & 113 & 117 & 119 & 112 & 114 & 102 & 101 & 102 & & & \\
\hline 12 & 122 & 119 & 121 & 113 & 109 & 111 & 102 & 101 & 101 & & & \\
\hline 13 & 121 & 118 & 120 & 114 & 111 & 112 & 102 & 101 & 101 & & & \\
\hline 14 & 121 & 117 & 119 & 111 & 104 & 108 & 102 & 101 & 101 & & & \\
\hline 15 & 120 & 115 & 118 & 107 & 104 & 105 & 102 & 100 & 101 & & & \\
\hline 16 & 115 & 109 & 111 & 107 & 104 & 106 & 100 & 99 & 99 & & & \\
\hline 17 & 111 & 108 & 109 & 108 & 103 & 106 & & & & & & \\
\hline 18 & 113 & 111 & 112 & 107 & 105 & 106 & & & & & & \\
\hline 19 & 115 & 111 & 114 & 109 & 107 & 108 & & & & & & \\
\hline 20 & 114 & 111 & 112 & 110 & 108 & 109 & & & & & & \\
\hline 21 & 115 & 113 & 114 & 113 & 109 & 111 & & & & & & \\
\hline 22 & 117 & 113 & 115 & 115 & 112 & 113 & & & & & & \\
\hline 23 & 118 & 116 & 117 & 115 & 113 & 114 & & & & & & \\
\hline 24 & 119 & 117 & 118 & 116 & 115 & 115 & & & & & & \\
\hline 25 & 118 & 116 & 117 & 116 & 114 & 115 & & & & & & \\
\hline 26 & 119 & 117 & 118 & 116 & 112 & 114 & & & & & & \\
\hline 27 & 119 & 117 & 118 & 113 & 106 & 109 & & & & & & \\
\hline 28 & 117 & 115 & 116 & 107 & 106 & 106 & & & & & & \\
\hline 29 & 116 & 114 & 114 & 112 & 107 & 110 & & & & & & \\
\hline 30 & 114 & 112 & 113 & 113 & 111 & 112 & & & & & & \\
\hline 31 & 112 & 109 & 111 & 113 & 109 & 111 & & & & & & \\
\hline Month & 123 & 108 & 116 & 121 & 103 & 111 & & & & & & \\
\hline
\end{tabular}



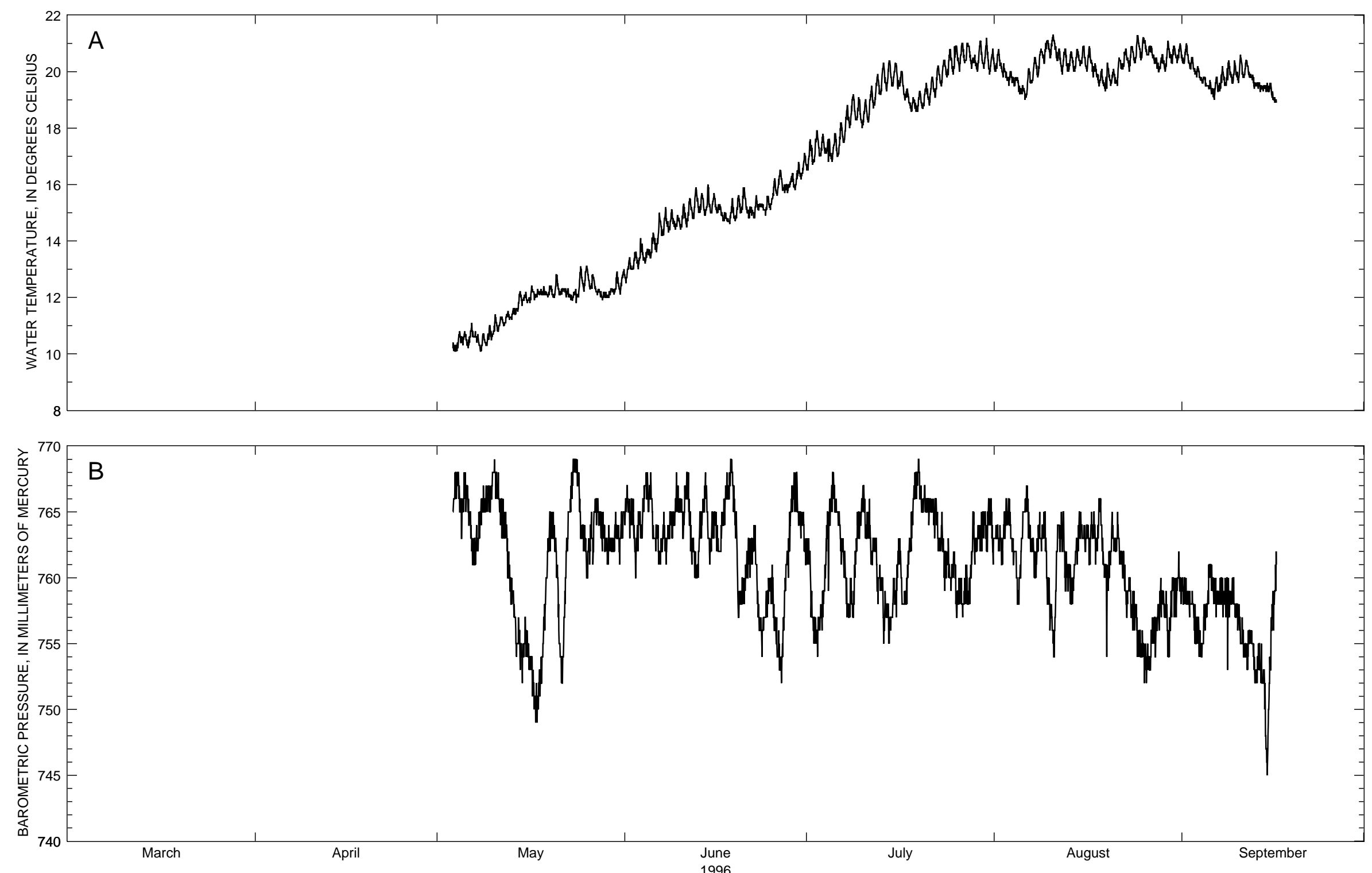

Figure 11. Hourly values of (A) water temperature, (B) barometric pressure, and (C, D) total dissolved gas at the Columbia River, right bank, near Skamania, Washington May - September 1996. 

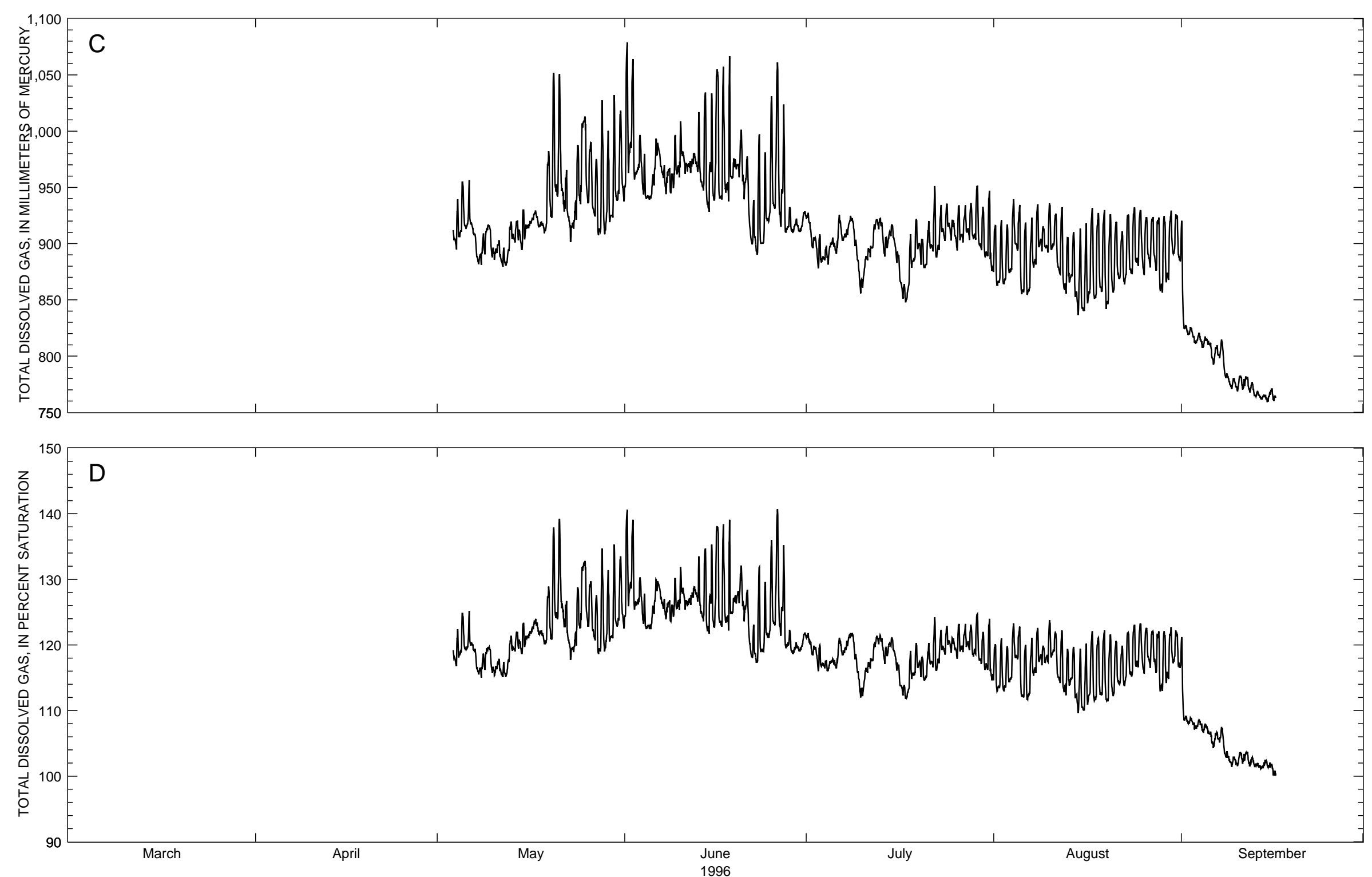

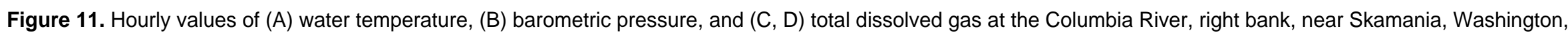
May - September 1996._continued 
Table 29. Daily summary of water temperature for the Columbia River, right bank, near Skamania, Washington, March - September 1996

[Values reported in degrees Celsius; Max, maximum; Min, minimum; Month, monthly summary statistics; --, not available; e, estimated]

\begin{tabular}{|c|c|c|c|c|c|c|c|c|c|c|c|c|}
\hline \multirow{2}{*}{ Day } & \multicolumn{3}{|c|}{ March } & \multicolumn{3}{|c|}{ April } & \multicolumn{3}{|c|}{ May } & \multicolumn{3}{|c|}{ June } \\
\hline & Max & Min & Mean & Max & Min & Mean & Max & Min & Mean & Max & Min & Mean \\
\hline 1 & & & & -- & -- & -- & -- & -- & -- & 13.4 & 12.5 & 13.0 \\
\hline 2 & & & & -- & -- & -- & -- & -- & -- & 13.6 & 13.0 & 13.3 \\
\hline 3 & & & & -- & -- & -- & -- & -- & -- & 14.1 & 13.0 & 13.5 \\
\hline 4 & & & & -- & -- & -- & 10.8 & 10.1 & 10.4 & 13.7 & 13.2 & 13.5 \\
\hline 5 & & & & -- & -- & -- & 10.8 & 10.3 & 10.5 & 14.3 & 13.4 & 13.8 \\
\hline 6 & & & & -- & -- & -- & 11.1 & 10.2 & 10.6 & 15.0 & 13.6 & 14.2 \\
\hline 7 & & & & -- & -- & -- & 10.8 & 10.3 & 10.6 & 15.2 & 14.2 & 14.6 \\
\hline 8 & & & & -- & -- & -- & 10.7 & 10.1 & 10.4 & 15.1 & 14.3 & 14.7 \\
\hline 9 & & & & -- & -- & -- & 11.0 & 10.3 & 10.6 & 14.9 & 14.4 & 14.7 \\
\hline 10 & & & & -- & -- & -- & 11.4 & 10.5 & 10.9 & 15.3 & 14.4 & 14.8 \\
\hline 11 & & & & -- & -- & -- & 11.3 & 10.8 & 11.1 & 15.5 & 14.5 & 15.0 \\
\hline 12 & & & & -- & -- & -- & 11.5 & 11.0 & 11.3 & 15.9 & 14.8 & 15.3 \\
\hline 13 & & & & -- & -- & -- & 11.6 & 11.2 & 11.4 & 15.7 & 15.0 & 15.3 \\
\hline 14 & & & & -- & -- & -- & 12.2 & 11.4 & 11.8 & 16.0 & 14.9 & 15.3 \\
\hline 15 & -- & -- & -- & -- & -- & -- & 12.2 & 11.7 & 11.9 & 15.7 & 15.0 & 15.3 \\
\hline 16 & -- & -- & -- & -- & -- & -- & 12.4 & 11.8 & 12.1 & 15.3 & 14.9 & 15.1 \\
\hline 17 & -- & -- & -- & -- & -- & -- & 12.3 & 11.9 & 12.1 & 15.0 & 14.7 & 14.8 \\
\hline 18 & -- & -- & -- & -- & -- & -- & 12.4 & 12.1 & 12.2 & 15.5 & 14.6 & 15.0 \\
\hline 19 & -- & -- & -- & -- & -- & -- & 12.4 & 12.0 & 12.2 & 15.6 & 14.7 & 15.1 \\
\hline 20 & -- & -- & -- & -- & -- & -- & 12.8 & 12.0 & 12.3 & 15.9 & 15.0 & 15.4 \\
\hline 21 & -- & -- & -- & -- & -- & -- & 12.3 & 12.1 & 12.2 & 15.3 & 14.8 & 15.1 \\
\hline 22 & -- & -- & -- & -- & -- & -- & 12.3 & 12.0 & 12.2 & 15.6 & 14.8 & 15.1 \\
\hline 23 & -- & -- & -- & -- & -- & -- & 12.3 & 11.8 & 12.1 & 15.3 & 15.1 & 15.2 \\
\hline 24 & -- & -- & -- & -- & -- & -- & 13.1 & 11.8 & 12.4 & 15.6 & 14.9 & 15.2 \\
\hline 25 & -- & -- & -- & -- & -- & -- & 13.1 & 12.2 & 12.7 & 16.2 & 15.1 & 15.7 \\
\hline 26 & -- & -- & -- & -- & -- & -- & 12.8 & 12.3 & 12.5 & 16.5 & 15.6 & 16.1 \\
\hline 27 & -- & -- & -- & -- & -- & -- & 12.5 & 12.0 & 12.2 & 16.0 & 15.7 & 15.9 \\
\hline 28 & -- & -- & -- & -- & -- & -- & 12.2 & 11.9 & 12.1 & 16.4 & 15.9 & 16.1 \\
\hline 29 & -- & -- & -- & -- & -- & -- & 12.3 & 12.0 & 12.1 & 16.8 & 15.8 & 16.3 \\
\hline 30 & -- & -- & -- & -- & -- & -- & 12.9 & 12.1 & 12.4 & 17.1 & 16.2 & 16.6 \\
\hline 31 & -- & -- & -- & -- & -- & -- & 13.0 & 12.1 & 12.6 & -- & -- & -- \\
\hline Month & -- & -- & -- & -- & -- & -- & -- & -- & -- & 17.1 & 12.5 & 15.0 \\
\hline \multirow{2}{*}{ Day } & \multicolumn{3}{|c|}{ July } & \multicolumn{3}{|c|}{$\overline{\text { August }}$} & \multicolumn{3}{|c|}{ September } & & & \\
\hline & Max & Min & Mean & Max & Min & Mean & Max & Min & Mean & & & \\
\hline 1 & 17.6 & 16.5 & 17.0 & 20.8 & 20.0 & 20.4 & 21.0 & 20.3 & 20.6 & & & \\
\hline 2 & 17.9 & 16.7 & 17.3 & 20.3 & 19.8 & 20.1 & 20.6 & 20.1 & 20.3 & & & \\
\hline 3 & 17.8 & 17.0 & 17.3 & 20.0 & 19.5 & 19.8 & 20.2 & 19.8 & 20.0 & & & \\
\hline 4 & 17.6 & 16.8 & 17.3 & 19.8 & 19.5 & 19.7 & 19.8 & 19.5 & 19.7 & & & \\
\hline 5 & 17.8 & 16.8 & 17.3 & 19.5 & 19.2 & 19.3 & 19.7 & 19.2 & 19.5 & & & \\
\hline 6 & 18.2 & 17.0 & 17.6 & 20.1 & 19.0 & 19.6 & 19.8 & 19.0 & 19.4 & & & \\
\hline 7 & 18.8 & 17.5 & 18.1 & 20.5 & 19.6 & 20.0 & 20.2 & 19.3 & 19.7 & & & \\
\hline 8 & 19.2 & 18.0 & 18.6 & 20.8 & 19.8 & 20.4 & 20.4 & 19.5 & 19.9 & & & \\
\hline 9 & 19.1 & 18.3 & 18.6 & 21.1 & 20.3 & 20.8 & 20.4 & 19.6 & 19.9 & & & \\
\hline 10 & 19.0 & 18.0 & 18.5 & 21.3 & 20.5 & 20.9 & 20.6 & 19.6 & 20.1 & & & \\
\hline 11 & -- & -- & -- & 21.0 & 20.3 & 20.6 & 20.4 & 19.8 & 20.1 & & & \\
\hline 12 & 19.9 & 18.7 & 19.3 & 20.8 & 19.9 & 20.4 & 20.0 & 19.4 & 19.8 & & & \\
\hline 13 & 20.3 & 19.2 & 19.7 & 20.7 & 20.0 & 20.4 & 19.6 & 19.3 & 19.5 & & & \\
\hline 14 & 20.4 & 19.3 & 19.9 & 20.8 & 20.0 & 20.4 & 19.5 & 19.3 & 19.4 & & & \\
\hline 15 & 20.3 & 19.5 & 19.9 & 20.9 & 20.2 & 20.5 & 19.6 & 19.1 & 19.4 & & & \\
\hline 16 & 20.0 & 19.4 & 19.7 & 20.9 & 20.0 & 20.4 & & & & & & \\
\hline 17 & 19.5 & 18.9 & 19.2 & 20.4 & 19.8 & 20.1 & & & & & & \\
\hline 18 & 19.1 & 18.6 & 18.8 & 20.1 & 19.5 & 19.8 & & & & & & \\
\hline 19 & 19.3 & 18.6 & 18.9 & 20.3 & 19.3 & 19.8 & & & & & & \\
\hline 20 & 19.6 & 18.7 & 19.1 & 20.1 & 19.5 & 19.8 & & & & & & \\
\hline 21 & 19.8 & 18.8 & 19.3 & 20.5 & 19.5 & 20.0 & & & & & & \\
\hline 22 & 20.2 & 19.1 & 19.7 & 20.8 & 20.1 & 20.4 & & & & & & \\
\hline 23 & 20.4 & 19.5 & 20.0 & 20.9 & 20.0 & 20.5 & & & & & & \\
\hline 24 & 20.8 & 19.8 & 20.3 & 21.3 & 20.3 & 20.8 & & & & & & \\
\hline 25 & 20.9 & 19.9 & 20.4 & 21.2 & 20.4 & 20.9 & & & & & & \\
\hline 26 & 21.0 & 20.0 & 20.6 & 20.9 & 20.6 & 20.7 & & & & & & \\
\hline 27 & 21.0 & 20.3 & 20.7 & 20.8 & 20.1 & 20.4 & & & & & & \\
\hline 28 & 20.6 & 20.1 & 20.4 & 20.6 & 20.0 & 20.3 & & & & & & \\
\hline 29 & 21.1 & 20.0 & 20.5 & 21.1 & 20.0 & 20.5 & & & & & & \\
\hline 30 & 21.2 & 20.1 & 20.6 & 20.9 & 20.3 & 20.6 & & & & & & \\
\hline 31 & 20.8 & 19.9 & 20.4 & 21.0 & 20.3 & 20.6 & & & & & & \\
\hline Month & -- & -- & -- & 21.3 & 19.0 & 20.3 & & & & & & \\
\hline
\end{tabular}


Table 30. Daily summary of barometric pressure for the Columbia River, right bank, near Skamania, Washington, March - September 1996

[Values reported in millimeters of mercury; Max, maximum; Min, minimum; Month, monthly summary statistics; --, not available; e, estimated]

\begin{tabular}{|c|c|c|c|c|c|c|c|c|c|c|c|c|}
\hline \multirow{2}{*}{ Day } & \multicolumn{3}{|c|}{ March } & \multicolumn{3}{|c|}{ April } & \multicolumn{3}{|c|}{ May } & \multicolumn{3}{|c|}{ June } \\
\hline & Max & Min & Mean & Max & Min & Mean & Max & Min & Mean & Max & Min & Mean \\
\hline 1 & & & & -- & -- & -- & -- & -- & -- & 767 & 763 & 765 \\
\hline 2 & & & & -- & -- & -- & -- & -- & -- & 766 & 760 & 764 \\
\hline 3 & & & & -- & -- & -- & -- & -- & -- & 766 & 762 & 764 \\
\hline 4 & & & & -- & -- & -- & 768 & 765 & 767 & 768 & 765 & 766 \\
\hline 5 & & & & -- & -- & -- & 768 & 763 & 766 & 768 & 763 & 765 \\
\hline 6 & & & & -- & -- & -- & 767 & 761 & 764 & 764 & 761 & 763 \\
\hline 7 & & & & -- & -- & -- & 765 & 761 & 763 & 765 & 761 & 763 \\
\hline 8 & & & & -- & -- & -- & 767 & 763 & 765 & 765 & 763 & 764 \\
\hline 9 & & & & -- & -- & -- & 767 & 765 & 766 & 768 & 764 & 765 \\
\hline 10 & & & & -- & -- & -- & 769 & 766 & 768 & 767 & 763 & 765 \\
\hline 11 & & & & -- & -- & -- & 768 & 763 & 765 & 768 & 762 & 766 \\
\hline 12 & & & & -- & -- & -- & 765 & 760 & 763 & 763 & 760 & 761 \\
\hline 13 & & & & -- & -- & -- & 761 & 756 & 758 & 766 & 760 & 764 \\
\hline 14 & & & & -- & -- & -- & 757 & 753 & 755 & 768 & 762 & 765 \\
\hline 15 & -- & -- & -- & -- & -- & -- & 757 & 752 & 755 & 765 & 761 & 764 \\
\hline 16 & -- & -- & -- & -- & -- & -- & 755 & 751 & 753 & 765 & 762 & 763 \\
\hline 17 & -- & -- & -- & -- & -- & -- & 753 & 749 & 751 & 768 & 764 & 765 \\
\hline 18 & -- & -- & -- & -- & -- & -- & 760 & 751 & 755 & 769 & 765 & 767 \\
\hline 19 & -- & -- & -- & -- & -- & -- & 765 & 760 & 763 & 767 & 757 & 762 \\
\hline 20 & -- & -- & -- & -- & -- & -- & 765 & 759 & 762 & 761 & 758 & 759 \\
\hline 21 & -- & -- & -- & -- & -- & -- & 759 & 752 & 755 & 763 & 760 & 762 \\
\hline 22 & -- & -- & -- & -- & -- & -- & 765 & 757 & 762 & 764 & 758 & 762 \\
\hline 23 & -- & -- & -- & -- & -- & -- & 769 & 765 & 768 & 758 & 754 & 756 \\
\hline 24 & -- & -- & -- & -- & -- & -- & 769 & 763 & 766 & 760 & 757 & 758 \\
\hline 25 & -- & -- & -- & -- & -- & -- & 764 & 760 & 762 & 761 & 755 & 758 \\
\hline 26 & -- & -- & -- & -- & -- & -- & 765 & 761 & 763 & 757 & 752 & 754 \\
\hline 27 & -- & -- & -- & -- & -- & -- & 766 & 764 & 765 & 762 & 754 & 759 \\
\hline 28 & -- & -- & -- & -- & -- & -- & 765 & 762 & 763 & 768 & 760 & 765 \\
\hline 29 & -- & -- & -- & -- & -- & -- & 764 & 761 & 763 & 768 & 764 & 766 \\
\hline 30 & -- & -- & -- & -- & -- & -- & 765 & 762 & 763 & 765 & 761 & 763 \\
\hline 31 & -- & -- & -- & -- & -- & -- & 765 & 761 & 764 & -- & -- & -- \\
\hline Month & -- & -- & -- & -- & -- & -- & -- & -- & -- & 769 & 752 & 763 \\
\hline \multirow{2}{*}{ Day } & \multicolumn{3}{|c|}{ July } & \multicolumn{3}{|c|}{ August } & \multicolumn{3}{|c|}{ September } & & & \\
\hline & Max & Min & Mean & Max & Min & Mean & Max & Min & Mean & & & \\
\hline 1 & 763 & 757 & 761 & 764 & 762 & 763 & 760 & 758 & 759 & & & \\
\hline 2 & 757 & 754 & 756 & 766 & 761 & 763 & 759 & 757 & 758 & & & \\
\hline 3 & 759 & 756 & 757 & 766 & 762 & 764 & 758 & 754 & 756 & & & \\
\hline 4 & 766 & 759 & 763 & 763 & 758 & 761 & 758 & 754 & 756 & & & \\
\hline 5 & 768 & 765 & 766 & 765 & 758 & 762 & 761 & 757 & 760 & & & \\
\hline 6 & 765 & 761 & 764 & 767 & 762 & 765 & 760 & 757 & 759 & & & \\
\hline 7 & 763 & 757 & 760 & 763 & 760 & 762 & 760 & 757 & 759 & & & \\
\hline 8 & 761 & 757 & 758 & 765 & 761 & 763 & 760 & 753 & 758 & & & \\
\hline 9 & 765 & 760 & 763 & 765 & 758 & 762 & 760 & 757 & 758 & & & \\
\hline 10 & 767 & 763 & 765 & 760 & 754 & 757 & 758 & 755 & 756 & & & \\
\hline 11 & 766 & 761 & 763 & 764 & 756 & 762 & 756 & 753 & 755 & & & \\
\hline 12 & 763 & 758 & 761 & 765 & 759 & 762 & 756 & 753 & 755 & & & \\
\hline 13 & 761 & 755 & 759 & 761 & 758 & 760 & 755 & 752 & 753 & & & \\
\hline 14 & 759 & 755 & 757 & 764 & 759 & 762 & 755 & 746 & 751 & & & \\
\hline 15 & 761 & 757 & 759 & 765 & 762 & 764 & 759 & 745 & 752 & & & \\
\hline 16 & 763 & 758 & 761 & 764 & 761 & 763 & & & & & & \\
\hline 17 & 763 & 758 & 759 & 765 & 761 & 763 & & & & & & \\
\hline 18 & 768 & 762 & 765 & 766 & 761 & 764 & & & & & & \\
\hline 19 & 769 & 765 & 767 & 762 & e754 & 760 & & & & & & \\
\hline 20 & 767 & 765 & 766 & 765 & 760 & 763 & & & & & & \\
\hline 21 & 766 & 764 & 765 & 765 & 761 & 763 & & & & & & \\
\hline 22 & 766 & 762 & 764 & 762 & 757 & 760 & & & & & & \\
\hline 23 & 765 & 759 & 762 & 760 & 756 & 758 & & & & & & \\
\hline 24 & 763 & 759 & 761 & 759 & 754 & 756 & & & & & & \\
\hline 25 & 762 & 757 & 760 & 756 & 752 & 755 & & & & & & \\
\hline 26 & 760 & 757 & 758 & 756 & 752 & 754 & & & & & & \\
\hline 27 & 761 & 758 & 759 & 757 & 754 & 756 & & & & & & \\
\hline 28 & 765 & 760 & 762 & 760 & 756 & 758 & & & & & & \\
\hline 29 & 765 & 762 & 763 & 759 & 754 & 756 & & & & & & \\
\hline 30 & 765 & 762 & 764 & 760 & 756 & 758 & & & & & & \\
\hline 31 & 766 & 761 & 764 & 762 & 757 & 759 & & & & & & \\
\hline Month & 769 & 754 & 762 & 767 & 752 & 761 & & & & & & \\
\hline
\end{tabular}


Table 31. Daily summary of total dissolved gas, in millimeters of mercury, for the Columbia River, right bank, near Skamania, Washington, March - September 1996

[Max, maximum; Min, minimum; Month, monthly summary statistics; --, not available]

\begin{tabular}{|c|c|c|c|c|c|c|c|c|c|c|c|c|}
\hline \multirow{2}{*}{ Day } & \multicolumn{3}{|c|}{ March } & \multicolumn{3}{|c|}{ April } & \multicolumn{3}{|c|}{ May } & \multicolumn{3}{|c|}{ June } \\
\hline & Max & Min & Mean & Max & Min & Mean & Max & Min & Mean & Max & Min & Mean \\
\hline 1 & & & & -- & -- & -- & -- & -- & -- & 1080 & 951 & 1000 \\
\hline 2 & & & & -- & -- & -- & -- & -- & -- & 1060 & 957 & 993 \\
\hline 3 & & & & -- & -- & -- & -- & -- & -- & 996 & 949 & 972 \\
\hline 4 & & & & -- & -- & -- & 939 & 895 & 912 & 980 & 940 & 948 \\
\hline 5 & & & & -- & -- & -- & 955 & 911 & 927 & 967 & 939 & 950 \\
\hline 6 & & & & -- & -- & -- & 956 & 909 & 923 & 993 & 966 & 981 \\
\hline 7 & & & & -- & -- & -- & 909 & 882 & 895 & 970 & 944 & 959 \\
\hline 8 & & & & -- & -- & -- & 909 & 881 & 895 & 967 & 945 & 959 \\
\hline 9 & & & & -- & -- & -- & 917 & 892 & 911 & 996 & 949 & 969 \\
\hline 10 & & & & -- & -- & -- & 899 & 886 & 893 & 1010 & 959 & 980 \\
\hline 11 & & & & -- & -- & -- & 903 & 879 & 891 & 973 & 962 & 969 \\
\hline 12 & & & & -- & -- & -- & 914 & 880 & 889 & 981 & 968 & 974 \\
\hline 13 & & & & -- & -- & -- & 919 & 901 & 909 & 1020 & 947 & 967 \\
\hline 14 & & & & -- & -- & -- & 920 & 895 & 909 & 1030 & 928 & 967 \\
\hline 15 & -- & -- & -- & -- & -- & -- & 930 & 895 & 914 & 1030 & 939 & 967 \\
\hline 16 & -- & -- & -- & -- & -- & -- & 925 & 914 & 919 & 1050 & 940 & 990 \\
\hline 17 & -- & -- & -- & -- & -- & -- & 929 & 914 & 922 & 1060 & 939 & 976 \\
\hline 18 & -- & -- & -- & -- & -- & -- & 919 & 909 & 915 & 1070 & 937 & 973 \\
\hline 19 & -- & -- & -- & -- & -- & -- & 982 & 915 & 946 & 976 & 959 & 970 \\
\hline 20 & -- & -- & -- & -- & -- & -- & 1050 & 927 & 973 & 1000 & 939 & 968 \\
\hline 21 & -- & -- & -- & -- & -- & -- & 1050 & 929 & 974 & 977 & 902 & 937 \\
\hline 22 & -- & -- & -- & -- & -- & -- & 965 & 913 & 934 & 939 & 890 & 906 \\
\hline 23 & -- & -- & -- & -- & -- & -- & 938 & 901 & 920 & 997 & 900 & 924 \\
\hline 24 & -- & -- & -- & -- & -- & -- & 1010 & 921 & 962 & 980 & 904 & 932 \\
\hline 25 & -- & -- & -- & -- & -- & -- & 1010 & 936 & 982 & 1030 & 928 & 962 \\
\hline 26 & -- & -- & -- & -- & -- & -- & 990 & 926 & 956 & 1060 & 915 & 974 \\
\hline 27 & -- & -- & -- & -- & -- & -- & 975 & 907 & 933 & 1020 & 910 & 940 \\
\hline 28 & -- & -- & -- & -- & -- & -- & 1030 & 908 & 947 & 932 & 910 & 917 \\
\hline 29 & -- & -- & -- & -- & -- & -- & 1000 & 919 & 942 & 922 & 910 & 916 \\
\hline 30 & -- & -- & -- & -- & -- & -- & 1030 & 923 & 959 & 929 & 911 & 921 \\
\hline 31 & -- & -- & -- & -- & -- & -- & 1020 & 937 & 969 & -- & -- & -- \\
\hline Month & -- & -- & -- & -- & -- & -- & -- & -- & -- & 1080 & 890 & 959 \\
\hline \multirow{2}{*}{ Day } & \multicolumn{3}{|c|}{ July } & \multicolumn{3}{|c|}{ August } & \multicolumn{3}{|c|}{ September } & & & \\
\hline & Max & Min & Mean & Max & Min & Mean & Max & Min & Mean & & & \\
\hline 1 & 927 & 897 & 917 & 916 & 863 & 882 & 920 & 822 & 844 & & & \\
\hline 2 & 905 & 878 & 897 & 921 & 864 & 882 & 826 & 817 & 821 & & & \\
\hline 3 & 908 & 878 & 890 & 917 & 872 & 888 & 820 & 811 & 815 & & & \\
\hline 4 & 898 & 881 & 892 & 939 & 894 & 910 & 819 & 807 & 813 & & & \\
\hline 5 & 905 & 891 & 899 & 934 & 855 & 885 & 816 & 801 & 811 & & & \\
\hline 6 & 925 & 891 & 911 & 918 & 855 & 877 & 809 & 793 & 801 & & & \\
\hline 7 & 917 & 903 & 908 & 923 & 879 & 892 & 814 & 795 & 805 & & & \\
\hline 8 & 925 & 903 & 918 & 935 & 893 & 909 & 795 & 775 & 782 & & & \\
\hline 9 & 903 & 856 & 885 & 915 & 892 & 902 & 780 & 771 & 776 & & & \\
\hline 10 & 889 & 856 & 874 & 936 & 894 & 908 & 782 & 769 & 776 & & & \\
\hline 11 & 898 & 885 & 893 & 926 & 873 & 896 & 782 & 770 & 777 & & & \\
\hline 12 & 921 & 897 & 915 & 932 & 855 & 884 & 779 & 767 & 772 & & & \\
\hline 13 & 922 & 888 & 910 & 905 & 866 & 880 & 769 & 763 & 766 & & & \\
\hline 14 & 911 & 888 & 904 & 910 & 836 & 869 & 765 & 761 & 764 & & & \\
\hline 15 & 917 & 883 & 903 & 913 & 840 & 861 & 771 & 759 & 765 & & & \\
\hline 16 & 893 & 851 & 873 & 918 & 847 & 871 & & & & & & \\
\hline 17 & 866 & 848 & 857 & 932 & 851 & 883 & & & & & & \\
\hline 18 & 908 & 866 & 888 & 927 & 857 & 886 & & & & & & \\
\hline 19 & 921 & 882 & 902 & 930 & 842 & 878 & & & & & & \\
\hline 20 & 904 & 879 & 888 & 926 & 856 & 885 & & & & & & \\
\hline 21 & 920 & 893 & 902 & 919 & 869 & 889 & & & & & & \\
\hline 22 & 951 & 888 & 907 & 915 & 864 & 885 & & & & & & \\
\hline 23 & 934 & 900 & 914 & 925 & 873 & 895 & & & & & & \\
\hline 24 & 936 & 903 & 918 & 932 & 880 & 901 & & & & & & \\
\hline 25 & 921 & 894 & 910 & 930 & 873 & 901 & & & & & & \\
\hline 26 & 934 & 898 & 912 & 923 & 879 & 900 & & & & & & \\
\hline 27 & 934 & 903 & 917 & 923 & 876 & 900 & & & & & & \\
\hline 28 & 935 & 903 & 915 & 923 & 856 & 885 & & & & & & \\
\hline 29 & 952 & 895 & 916 & 924 & 866 & 890 & & & & & & \\
\hline 30 & 933 & 888 & 903 & 929 & 891 & 907 & & & & & & \\
\hline 31 & 947 & 875 & 899 & 926 & 884 & 904 & & & & & & \\
\hline Month & 952 & 848 & 901 & 939 & 836 & 890 & & & & & & \\
\hline
\end{tabular}


Table 32. Daily summary of total dissolved gas, in percent saturation, for the Columbia River, right bank, near Skamania, Washington, March - September 1996

[Max, maximum; Min, minimum; Month, monthly summary statistics; --, not available]

\begin{tabular}{|c|c|c|c|c|c|c|c|c|c|c|c|c|}
\hline \multirow{2}{*}{ Day } & \multicolumn{3}{|c|}{ March } & \multicolumn{3}{|c|}{ April } & \multicolumn{3}{|c|}{ May } & \multicolumn{3}{|c|}{ June } \\
\hline & Max & Min & Mean & Max & Min & Mean & Max & Min & Mean & Max & Min & Mean \\
\hline 1 & & & & -- & -- & -- & -- & -- & -- & 141 & 124 & 131 \\
\hline 2 & & & & -- & -- & -- & -- & -- & -- & 139 & 125 & 130 \\
\hline 3 & & & & -- & -- & -- & -- & -- & -- & 130 & 124 & 127 \\
\hline 4 & & & & -- & -- & -- & 122 & 117 & 119 & 128 & 122 & 124 \\
\hline 5 & & & & -- & -- & -- & 125 & 119 & 121 & 127 & 122 & 124 \\
\hline 6 & & & & -- & -- & -- & 125 & 119 & 121 & 130 & 127 & 129 \\
\hline 7 & & & & -- & -- & -- & 119 & 116 & 117 & 127 & 124 & 126 \\
\hline 8 & & & & -- & -- & -- & 119 & 115 & 117 & 127 & 124 & 126 \\
\hline 9 & & & & -- & -- & -- & 120 & 116 & 119 & 130 & 124 & 127 \\
\hline 10 & & & & -- & -- & -- & 117 & 115 & 116 & 132 & 125 & 128 \\
\hline 11 & & & & -- & -- & -- & 118 & 115 & 116 & 128 & 125 & 127 \\
\hline 12 & & & & -- & -- & -- & 120 & 115 & 117 & 129 & 127 & 128 \\
\hline 13 & & & & -- & -- & -- & 121 & 119 & 120 & 133 & 124 & 127 \\
\hline 14 & & & & -- & -- & -- & 122 & 119 & 120 & 135 & 122 & 126 \\
\hline 15 & -- & -- & -- & -- & -- & -- & 123 & 119 & 121 & 135 & 123 & 127 \\
\hline 16 & -- & -- & -- & -- & -- & -- & 123 & 121 & 122 & 138 & 123 & 130 \\
\hline 17 & -- & -- & -- & -- & -- & -- & 124 & 122 & 123 & 138 & 123 & 128 \\
\hline 18 & -- & -- & -- & -- & -- & -- & 122 & 120 & 121 & 139 & 122 & 127 \\
\hline 19 & -- & -- & -- & -- & -- & -- & 129 & 120 & 124 & 128 & 126 & 127 \\
\hline 20 & -- & -- & -- & -- & -- & -- & 138 & 121 & 128 & 132 & 124 & 128 \\
\hline 21 & -- & -- & -- & -- & -- & -- & 139 & 123 & 129 & 128 & 118 & 123 \\
\hline 22 & -- & -- & -- & -- & -- & -- & 127 & 119 & 123 & 123 & 117 & 119 \\
\hline 23 & -- & -- & -- & -- & -- & -- & 122 & 118 & 120 & 132 & 119 & 122 \\
\hline 24 & -- & -- & -- & -- & -- & -- & 132 & 120 & 126 & 129 & 119 & 123 \\
\hline 25 & -- & -- & -- & -- & -- & -- & 133 & 123 & 129 & 136 & 122 & 127 \\
\hline 26 & -- & -- & -- & -- & -- & -- & 130 & 121 & 125 & 141 & 121 & 129 \\
\hline 27 & -- & -- & -- & -- & -- & -- & 128 & 119 & 122 & 135 & 119 & 124 \\
\hline 28 & -- & -- & -- & -- & -- & -- & 135 & 119 & 124 & 122 & 119 & 120 \\
\hline 29 & -- & -- & -- & -- & -- & -- & 131 & 121 & 124 & 120 & 119 & 120 \\
\hline 30 & -- & -- & -- & -- & -- & -- & 135 & 121 & 126 & 122 & 119 & 121 \\
\hline 31 & -- & -- & -- & -- & -- & -- & 133 & 122 & 127 & -- & -- & -- \\
\hline Month & -- & -- & -- & -- & -- & -- & -- & -- & -- & 141 & 117 & 126 \\
\hline \multirow{2}{*}{ Day } & \multicolumn{3}{|c|}{ July } & \multicolumn{3}{|c|}{ August } & \multicolumn{3}{|c|}{ September } & & & \\
\hline & Max & Min & Mean & Max & Min & Mean & Max & Min & Mean & & & \\
\hline 1 & 122 & 118 & 121 & 120 & 113 & 116 & 121 & 108 & 111 & & & \\
\hline 2 & 120 & 116 & 119 & 121 & 113 & 116 & 109 & 108 & 108 & & & \\
\hline 3 & 120 & 116 & 118 & 120 & 114 & 116 & 109 & 107 & 108 & & & \\
\hline 4 & 118 & 116 & 117 & 123 & 117 & 120 & 108 & 107 & 108 & & & \\
\hline 5 & 118 & 116 & 117 & 123 & 112 & 116 & 108 & 105 & 107 & & & \\
\hline 6 & 121 & 116 & 119 & 120 & 112 & 115 & 107 & 104 & 106 & & & \\
\hline 7 & 121 & 118 & 120 & 121 & 115 & 117 & 107 & 105 & 106 & & & \\
\hline 8 & 122 & 119 & 121 & 123 & 117 & 119 & 105 & 102 & 103 & & & \\
\hline 9 & 119 & 112 & 116 & 120 & 117 & 118 & 103 & 101 & 102 & & & \\
\hline 10 & 116 & 112 & 114 & 124 & 118 & 120 & 104 & 102 & 103 & & & \\
\hline 11 & 118 & 116 & 117 & 122 & 114 & 118 & 104 & 102 & 103 & & & \\
\hline 12 & 121 & 118 & 120 & 122 & 112 & 116 & 103 & 101 & 102 & & & \\
\hline 13 & 121 & 117 & 120 & 119 & 114 & 116 & 102 & 101 & 102 & & & \\
\hline 14 & 120 & 117 & 119 & 120 & 110 & 114 & 102 & 101 & 102 & & & \\
\hline 15 & 121 & 116 & 119 & 119 & 110 & 113 & 102 & 101 & 102 & & & \\
\hline 16 & 117 & 112 & 115 & 120 & 111 & 114 & & & & & & \\
\hline 17 & 114 & 112 & 113 & 122 & 111 & 116 & & & & & & \\
\hline 18 & 119 & 113 & 116 & 121 & 112 & 116 & & & & & & \\
\hline 19 & 120 & 115 & 118 & 122 & 111 & 116 & & & & & & \\
\hline 20 & 118 & 115 & 116 & 121 & 112 & 116 & & & & & & \\
\hline 21 & 120 & 117 & 118 & 121 & 114 & 117 & & & & & & \\
\hline 22 & 124 & 116 & 119 & 121 & 114 & 116 & & & & & & \\
\hline 23 & 122 & 118 & 120 & 122 & 115 & 118 & & & & & & \\
\hline 24 & 123 & 119 & 121 & 123 & 117 & 119 & & & & & & \\
\hline 25 & 121 & 118 & 120 & 123 & 116 & 119 & & & & & & \\
\hline 26 & 123 & 118 & 120 & 123 & 117 & 119 & & & & & & \\
\hline 27 & 123 & 119 & 121 & 122 & 116 & 119 & & & & & & \\
\hline 28 & 123 & 118 & 120 & 122 & 113 & 117 & & & & & & \\
\hline 29 & 125 & 117 & 120 & 122 & 114 & 118 & & & & & & \\
\hline 30 & 122 & 116 & 118 & 123 & 117 & 120 & & & & & & \\
\hline 31 & 124 & 115 & 118 & 122 & 116 & 119 & & & & & & \\
\hline Month & 125 & 112 & 118 & 124 & 110 & 117 & & & & & & \\
\hline
\end{tabular}



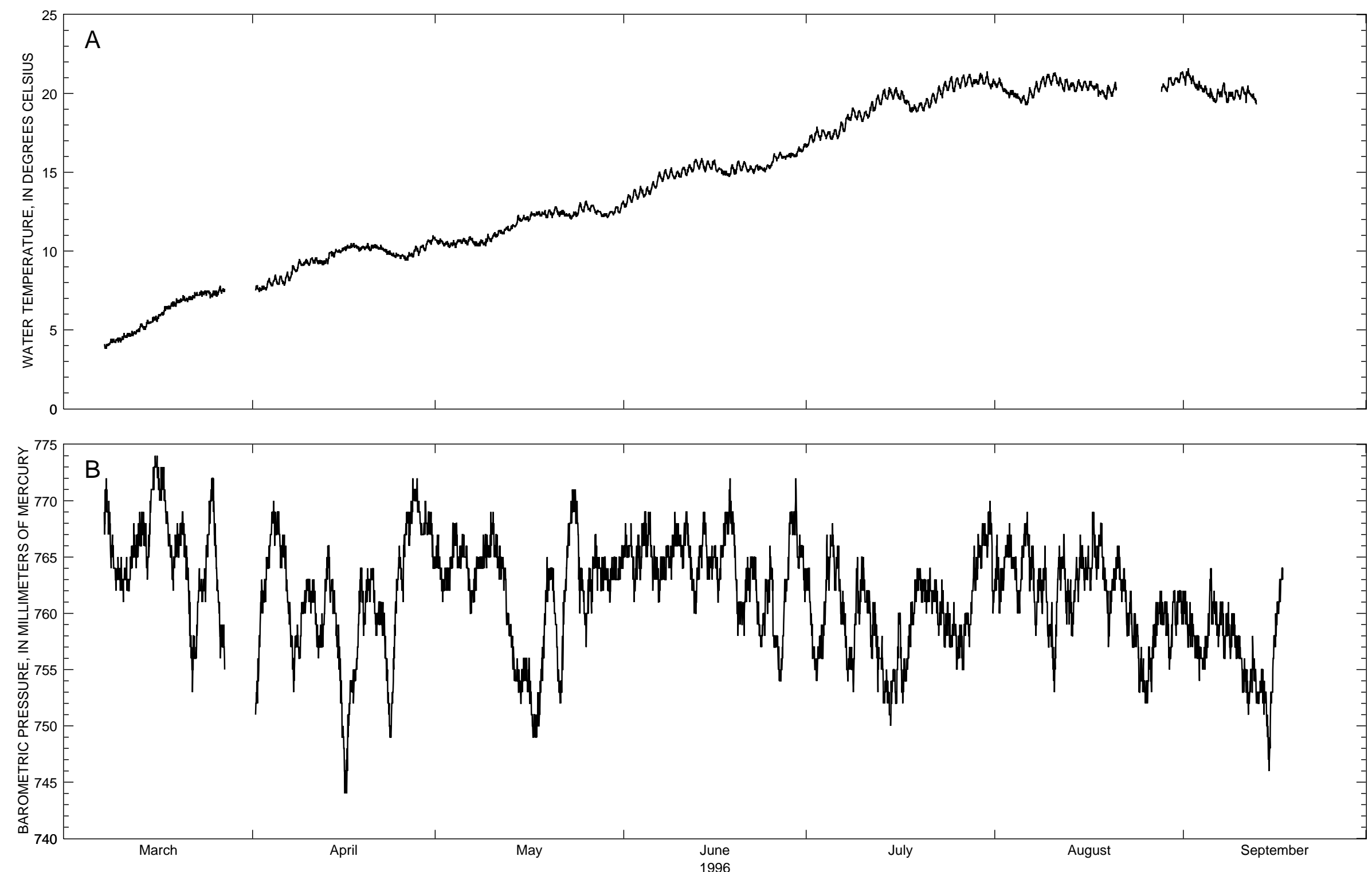

Figure 12. Hourly values of (A) water temperature, (B) barometric pressure, and (C, D) total dissolved gas at the Columbia River, left bank, near Dodson, Oregon, March September 1996. 


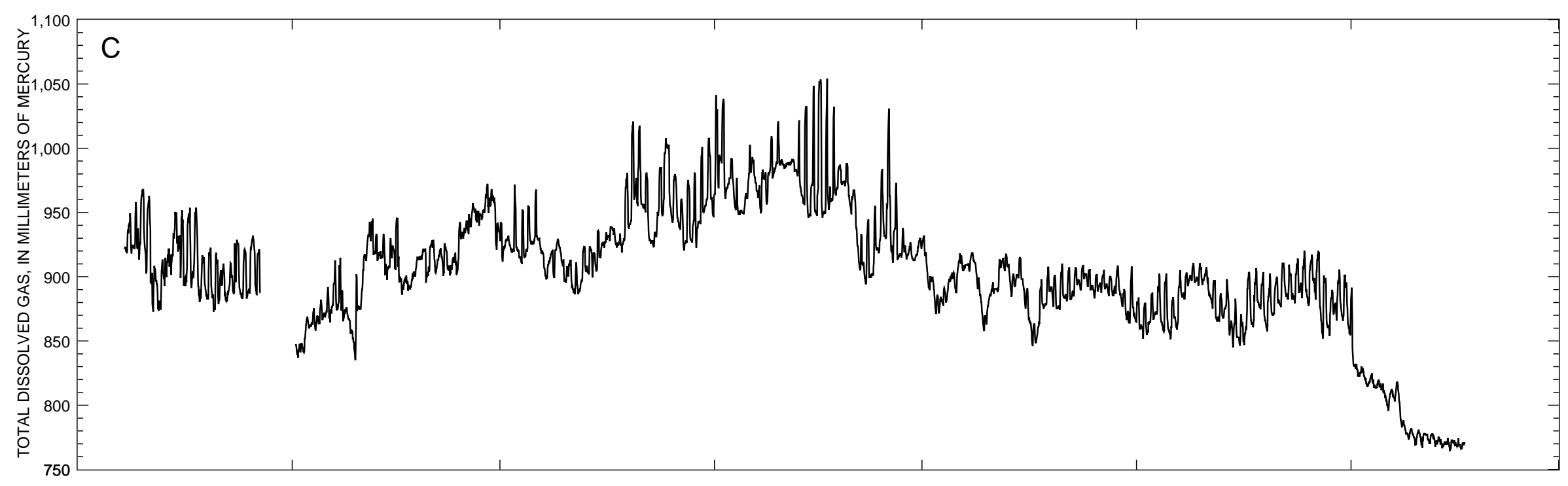

¿

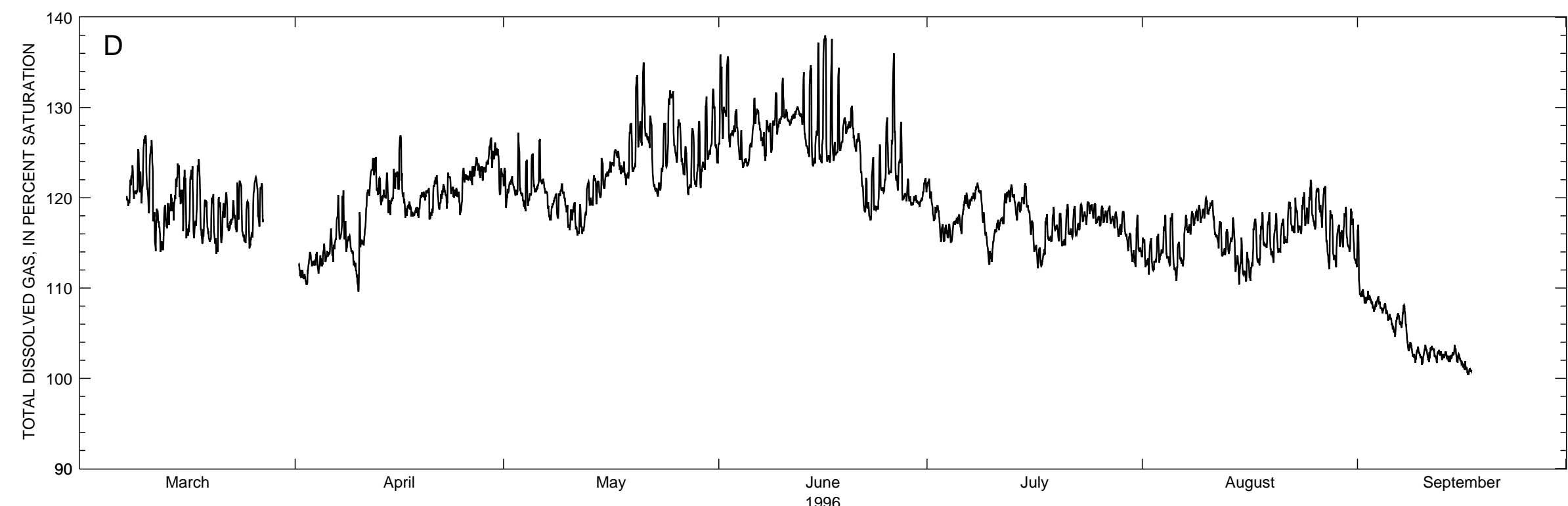

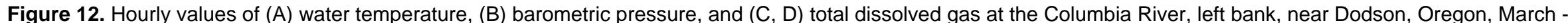
September 1996.-Continued 
Table 33. Daily summary of water temperature for the Columbia River, left bank, near Dodson, Oregon, March - September 1996

[Values reported in degrees Celsius; Max, maximum; Min, minimum; Month, monthly summary statistics; --, not available]

\begin{tabular}{|c|c|c|c|c|c|c|c|c|c|c|c|c|}
\hline \multirow{2}{*}{ Day } & \multicolumn{3}{|c|}{ March } & \multicolumn{3}{|c|}{ April } & \multicolumn{3}{|c|}{ May } & \multicolumn{3}{|c|}{ June } \\
\hline & Max & Min & Mean & Max & Min & Mean & Max & Min & Mean & Max & Min & Mean \\
\hline 1 & & & & -- & -- & -- & 10.8 & 10.4 & 10.6 & 13.6 & 12.8 & 13.1 \\
\hline 2 & & & & 7.8 & 7.4 & 7.6 & 10.7 & 10.3 & 10.5 & 13.9 & 13.1 & 13.5 \\
\hline 3 & & & & 8.3 & 7.5 & 7.9 & 10.6 & 10.2 & 10.4 & 14.1 & 13.3 & 13.6 \\
\hline 4 & & & & 8.5 & 7.8 & 8.1 & 10.7 & 10.2 & 10.5 & 14.1 & 13.4 & 13.7 \\
\hline 5 & & & & 8.4 & 7.9 & 8.1 & 10.8 & 10.5 & 10.6 & 14.4 & 13.6 & 14.0 \\
\hline 6 & & & & 8.7 & 7.8 & 8.2 & 10.9 & 10.4 & 10.7 & 15.0 & 14.0 & 14.4 \\
\hline 7 & & & & 9.1 & 8.1 & 8.6 & 10.8 & 10.3 & 10.5 & 15.2 & 14.4 & 14.8 \\
\hline 8 & 4.4 & 4.0 & 4.2 & 9.5 & 8.7 & 9.0 & 10.7 & 10.3 & 10.5 & 15.3 & 14.6 & 14.9 \\
\hline 9 & 4.5 & 4.2 & 4.3 & 9.5 & 9.1 & 9.2 & 11.1 & 10.3 & 10.7 & 15.1 & 14.6 & 14.8 \\
\hline 10 & 4.8 & 4.2 & 4.5 & 9.6 & 9.1 & 9.3 & 11.2 & 10.6 & 10.9 & 15.3 & 14.7 & 15.0 \\
\hline 11 & 4.8 & 4.5 & 4.6 & 9.6 & 9.2 & 9.4 & 11.3 & 10.9 & 11.2 & 15.5 & 14.8 & 15.1 \\
\hline 12 & 5.0 & 4.6 & 4.8 & 9.4 & 9.1 & 9.3 & 11.6 & 11.1 & 11.3 & 15.8 & 15.0 & 15.4 \\
\hline 13 & 5.4 & 4.9 & 5.1 & 10.0 & 9.2 & 9.6 & 11.7 & 11.3 & 11.5 & 15.9 & 15.2 & 15.5 \\
\hline 14 & 5.6 & 5.0 & 5.3 & 10.1 & 9.6 & 9.9 & 12.3 & 11.5 & 11.9 & 15.7 & 15.0 & 15.4 \\
\hline 15 & 5.8 & 5.4 & 5.6 & 10.2 & 9.9 & 10.0 & 12.3 & 11.9 & 12.0 & 15.8 & 15.2 & 15.5 \\
\hline 16 & 6.0 & 5.5 & 5.8 & 10.4 & 10.0 & 10.2 & 12.5 & 11.9 & 12.1 & 15.5 & 15.0 & 15.2 \\
\hline 17 & 6.5 & 5.9 & 6.2 & 10.5 & 10.2 & 10.3 & 12.5 & 12.2 & 12.3 & 15.2 & 14.8 & 15.0 \\
\hline 18 & 6.8 & 6.3 & 6.5 & 10.4 & 10.0 & 10.2 & 12.5 & 12.2 & 12.4 & 15.5 & 14.7 & 15.0 \\
\hline 19 & 7.0 & 6.5 & 6.7 & 10.5 & 10.1 & 10.2 & 12.6 & 12.1 & 12.4 & 15.7 & 14.9 & 15.3 \\
\hline 20 & 7.2 & 6.7 & 6.9 & 10.4 & 10.0 & 10.2 & 12.8 & 12.1 & 12.5 & 15.7 & 15.1 & 15.4 \\
\hline 21 & 7.1 & 6.8 & 7.0 & 10.4 & 10.1 & 10.2 & 12.7 & 12.2 & 12.4 & 15.5 & 15.0 & 15.2 \\
\hline 22 & 7.4 & 6.9 & 7.2 & 10.4 & 9.9 & 10.1 & 12.6 & 12.2 & 12.3 & 15.5 & 14.9 & 15.2 \\
\hline 23 & 7.5 & 7.1 & 7.3 & 10.1 & 9.7 & 9.9 & 12.5 & 12.0 & 12.2 & 15.4 & 15.1 & 15.2 \\
\hline 24 & 7.5 & 7.2 & 7.4 & 9.9 & 9.6 & 9.7 & 13.1 & 12.2 & 12.6 & 15.5 & 15.0 & 15.3 \\
\hline 25 & 7.5 & 7.0 & 7.2 & 9.8 & 9.5 & 9.7 & 13.2 & 12.5 & 12.8 & 16.2 & 15.2 & 15.7 \\
\hline 26 & 7.8 & 7.1 & 7.4 & 9.9 & 9.4 & 9.6 & 12.9 & 12.5 & 12.8 & 16.3 & 15.7 & 16.0 \\
\hline 27 & -- & -- & -- & 10.3 & 9.6 & 9.9 & 12.9 & 12.3 & 12.6 & 16.1 & 15.8 & 16.0 \\
\hline 28 & -- & -- & -- & 10.4 & 9.7 & 10.1 & 12.5 & 12.2 & 12.3 & 16.2 & 15.9 & 16.1 \\
\hline 29 & -- & -- & -- & 10.7 & 10.0 & 10.3 & 12.5 & 12.1 & 12.3 & 16.6 & 16.0 & 16.2 \\
\hline 30 & -- & -- & -- & 11.0 & 10.4 & 10.7 & 12.8 & 12.3 & 12.6 & 16.8 & 16.3 & 16.5 \\
\hline 31 & -- & -- & -- & -- & -- & -- & 13.2 & 12.4 & 12.8 & -- & -- & -- \\
\hline Month & -- & -- & -- & -- & -- & -- & 13.2 & 10.2 & 11.7 & 16.8 & 12.8 & 15.1 \\
\hline \multirow{2}{*}{ Day } & \multicolumn{3}{|c|}{ July } & \multicolumn{3}{|c|}{ August } & \multicolumn{3}{|c|}{ September } & & & \\
\hline & Max & Min & Mean & Max & Min & Mean & Max & Min & Mean & & & \\
\hline 1 & 17.4 & 16.6 & 17.0 & 21.0 & 20.4 & 20.6 & 21.6 & 20.5 & 21.1 & & & \\
\hline 2 & 17.9 & 16.9 & 17.3 & 20.7 & 20.0 & 20.3 & 21.1 & 20.4 & 20.8 & & & \\
\hline 3 & 17.7 & 17.0 & 17.4 & 20.2 & 19.7 & 20.0 & 20.8 & 20.2 & 20.4 & & & \\
\hline 4 & 17.6 & 17.1 & 17.4 & 20.2 & 19.7 & 19.8 & 20.5 & 19.9 & 20.2 & & & \\
\hline 5 & 17.7 & 17.1 & 17.4 & 19.9 & 19.4 & 19.6 & 20.3 & 19.5 & 19.9 & & & \\
\hline 6 & 18.2 & 17.1 & 17.6 & 20.4 & 19.3 & 19.7 & 20.3 & 19.4 & 19.8 & & & \\
\hline 7 & 18.7 & 17.6 & 18.1 & 20.8 & 19.7 & 20.2 & 20.7 & 19.8 & 20.2 & & & \\
\hline 8 & 19.1 & 18.3 & 18.7 & 21.1 & 20.1 & 20.5 & 20.1 & 19.4 & 19.8 & & & \\
\hline 9 & 18.9 & 18.3 & 18.6 & 21.2 & 20.4 & 20.9 & 20.2 & 19.6 & 19.9 & & & \\
\hline 10 & 18.9 & 18.2 & 18.5 & 21.3 & 20.5 & 21.0 & 20.4 & 19.6 & 20.0 & & & \\
\hline 11 & 19.5 & 18.4 & 18.9 & 21.1 & 20.5 & 20.8 & 20.5 & 19.4 & 20.0 & & & \\
\hline 12 & 19.9 & 18.9 & 19.4 & 20.9 & 20.1 & 20.6 & 20.0 & 19.3 & 19.7 & & & \\
\hline 13 & 20.3 & 19.3 & 19.7 & 20.7 & 20.2 & 20.5 & -- & -- & -- & & & \\
\hline 14 & 20.4 & 19.6 & 19.9 & 20.9 & 20.1 & 20.4 & -- & -- & -- & & & \\
\hline 15 & 20.4 & 19.6 & 20.0 & 20.8 & 20.2 & 20.5 & -- & -- & -- & & & \\
\hline 16 & 20.2 & 19.6 & 19.9 & 20.8 & 20.2 & 20.5 & -- & -- & -- & & & \\
\hline 17 & 19.9 & 19.2 & 19.5 & 20.6 & 20.0 & 20.4 & & & & & & \\
\hline 18 & 19.3 & 18.8 & 19.0 & 20.2 & 19.8 & 20.1 & & & & & & \\
\hline 19 & 19.4 & 18.8 & 19.1 & 20.5 & 19.6 & 20.0 & & & & & & \\
\hline 20 & 19.7 & 18.9 & 19.3 & 20.7 & 19.8 & 20.2 & & & & & & \\
\hline 21 & 20.0 & 19.1 & 19.5 & -- & -- & -- & & & & & & \\
\hline 22 & 20.5 & 19.4 & 19.9 & -- & -- & -- & & & & & & \\
\hline 23 & 20.8 & 19.7 & 20.3 & -- & -- & -- & & & & & & \\
\hline 24 & 21.1 & 20.2 & 20.6 & -- & -- & -- & & & & & & \\
\hline 25 & 21.0 & 20.1 & 20.6 & -- & -- & -- & & & & & & \\
\hline 26 & 21.2 & 20.3 & 20.8 & -- & -- & -- & & & & & & \\
\hline 27 & 21.2 & 20.4 & 20.8 & -- & -- & -- & & & & & & \\
\hline 28 & 21.0 & 20.4 & 20.7 & -- & -- & -- & & & & & & \\
\hline 29 & 21.3 & 20.6 & 20.9 & 21.0 & 20.1 & 20.6 & & & & & & \\
\hline 30 & 21.4 & 20.6 & 20.8 & 21.0 & 20.5 & 20.8 & & & & & & \\
\hline 31 & 20.9 & 20.3 & 20.6 & 21.4 & 20.7 & 21.0 & & & & & & \\
\hline Month & 21.4 & 16.6 & 19.3 & -- & -- & -- & & & & & & \\
\hline
\end{tabular}


Table 34. Daily summary of barometric pressure for the Columbia River, left bank, near Dodson, Oregon, March - September 1996

[Values reported in millimeters of mercury; Max, maximum; Min, minimum; Month, monthly summary statistics; --, not available]

\begin{tabular}{|c|c|c|c|c|c|c|c|c|c|c|c|c|}
\hline \multirow{2}{*}{ Day } & \multicolumn{3}{|c|}{ March } & \multicolumn{3}{|c|}{ April } & \multicolumn{3}{|c|}{ May } & \multicolumn{3}{|c|}{ June } \\
\hline & Max & Min & Mean & Max & Min & Mean & Max & Min & Mean & Max & Min & Mean \\
\hline 1 & & & & -- & -- & -- & 767 & 763 & 765 & 768 & 765 & 766 \\
\hline 2 & & & & 763 & 755 & 760 & 764 & 761 & 763 & 768 & 761 & 764 \\
\hline 3 & & & & 767 & 761 & 764 & 768 & 762 & 764 & 768 & 763 & 765 \\
\hline 4 & & & & 770 & 766 & 768 & 768 & 764 & 766 & 769 & 765 & 767 \\
\hline 5 & & & & 769 & 764 & 767 & 767 & 764 & 765 & 769 & 762 & 766 \\
\hline 6 & & & & 767 & 760 & 763 & 766 & 760 & 763 & 766 & 761 & 764 \\
\hline 7 & & & & 761 & 753 & 757 & 765 & 761 & 763 & 766 & 763 & 764 \\
\hline 8 & 771 & 764 & 767 & 760 & 756 & 757 & 767 & 764 & 765 & 767 & 763 & 765 \\
\hline 9 & 766 & 762 & 764 & 763 & 760 & 761 & 767 & 764 & 766 & 769 & 765 & 767 \\
\hline 10 & 765 & 761 & 763 & 763 & 760 & 762 & 769 & 765 & 767 & 768 & 764 & 766 \\
\hline 11 & 765 & 762 & 763 & 763 & 756 & 759 & 767 & 763 & 765 & 769 & 763 & 767 \\
\hline 12 & 768 & 764 & 765 & 763 & 757 & 759 & 765 & 759 & 762 & 765 & 760 & 762 \\
\hline 13 & 769 & 765 & 767 & 766 & 761 & 763 & 760 & 755 & 758 & 767 & 762 & 765 \\
\hline 14 & 769 & 763 & 766 & 763 & 757 & 760 & 756 & 753 & 754 & 769 & 763 & 766 \\
\hline 15 & 774 & 766 & 771 & 759 & 748 & 753 & 756 & 752 & 754 & 766 & 763 & 765 \\
\hline 16 & 774 & 770 & 772 & 752 & 744 & 748 & 756 & 750 & 753 & 766 & 761 & 764 \\
\hline 17 & 773 & 768 & 770 & 755 & 752 & 754 & 753 & 749 & 750 & 769 & 765 & 767 \\
\hline 18 & 769 & 762 & 766 & 764 & 755 & 760 & 760 & 750 & 755 & 772 & 767 & 769 \\
\hline 19 & 768 & 764 & 766 & 764 & 758 & 761 & 765 & 759 & 762 & 768 & 758 & 763 \\
\hline 20 & 769 & 765 & 767 & 764 & 761 & 763 & 764 & 757 & 762 & 762 & 758 & 760 \\
\hline 21 & 766 & 756 & 761 & 762 & 758 & 760 & 758 & 752 & 754 & 765 & 761 & 763 \\
\hline 22 & 761 & 753 & 757 & 761 & 755 & 759 & 768 & 755 & 763 & 765 & 759 & 763 \\
\hline 23 & 764 & 761 & 762 & 756 & 749 & 752 & 771 & 766 & 769 & 761 & 757 & 759 \\
\hline 24 & 770 & 761 & 765 & 764 & 752 & 759 & 771 & 760 & 766 & 763 & 758 & 761 \\
\hline 25 & 772 & 765 & 769 & 767 & 761 & 764 & 764 & 757 & 761 & 766 & 757 & 761 \\
\hline 26 & 766 & 756 & 760 & 769 & 765 & 768 & 765 & 760 & 762 & 758 & 754 & 756 \\
\hline 27 & -- & -- & -- & 772 & 768 & 770 & 767 & 762 & 765 & 763 & 755 & 761 \\
\hline 28 & -- & -- & -- & 772 & 767 & 768 & 765 & 762 & 764 & 769 & 763 & 767 \\
\hline 29 & -- & -- & -- & 770 & 767 & 768 & 764 & 761 & 763 & 772 & 764 & 767 \\
\hline 30 & -- & -- & -- & 769 & 764 & 766 & 765 & 763 & 764 & 766 & 761 & 764 \\
\hline 31 & -- & -- & -- & -- & -- & -- & 766 & 763 & 764 & -- & -- & -- \\
\hline Month & -- & -- & -- & -- & -- & -- & 771 & 749 & 762 & 772 & 754 & 764 \\
\hline \multirow{2}{*}{ Day } & \multicolumn{3}{|c|}{ July } & \multicolumn{3}{|c|}{ August } & \multicolumn{3}{|c|}{ September } & & & \\
\hline & Max & Min & Mean & Max & Min & Mean & Max & Min & Mean & & & \\
\hline 1 & 764 & 758 & 762 & 767 & 760 & 763 & 762 & 757 & 760 & & & \\
\hline 2 & 759 & 754 & 756 & 766 & 761 & 764 & 761 & 756 & 758 & & & \\
\hline 3 & 761 & 756 & 757 & 768 & 764 & 765 & 758 & 754 & 757 & & & \\
\hline 4 & 767 & 759 & 763 & 765 & 760 & 763 & 758 & 755 & 756 & & & \\
\hline 5 & 768 & 762 & 765 & 768 & 760 & 764 & 764 & 757 & 761 & & & \\
\hline 6 & 766 & 759 & 762 & 769 & 762 & 766 & 762 & 757 & 760 & & & \\
\hline 7 & 762 & 754 & 757 & 766 & 758 & 762 & 761 & 756 & 759 & & & \\
\hline 8 & 759 & 753 & 756 & 764 & 760 & 762 & 761 & 756 & 758 & & & \\
\hline 9 & 765 & 759 & 761 & 766 & 757 & 761 & 760 & 756 & 758 & & & \\
\hline 10 & 765 & 761 & 763 & 761 & 753 & 757 & 758 & 753 & 756 & & & \\
\hline 11 & 762 & 756 & 759 & 766 & 756 & 763 & 756 & 751 & 753 & & & \\
\hline 12 & 761 & 753 & 758 & 767 & 759 & 763 & 758 & 752 & 755 & & & \\
\hline 13 & 758 & 752 & 755 & 763 & 758 & 761 & 755 & 752 & 753 & & & \\
\hline 14 & 755 & 750 & 753 & 765 & 761 & 763 & 755 & 747 & 752 & & & \\
\hline 15 & 755 & 752 & 754 & 767 & 762 & 764 & 758 & 746 & 752 & & & \\
\hline 16 & 760 & 752 & 757 & 766 & 762 & 764 & 763 & 757 & 760 & & & \\
\hline 17 & 759 & 753 & 756 & 769 & 763 & 766 & & & & & & \\
\hline 18 & 763 & 757 & 760 & 768 & 763 & 766 & & & & & & \\
\hline 19 & 764 & 761 & 763 & 764 & 757 & 761 & & & & & & \\
\hline 20 & 763 & 760 & 762 & 765 & 760 & 763 & & & & & & \\
\hline 21 & 764 & 760 & 762 & 766 & 761 & 764 & & & & & & \\
\hline 22 & 763 & 758 & 761 & 764 & 758 & 761 & & & & & & \\
\hline 23 & 763 & 757 & 760 & 762 & 757 & 759 & & & & & & \\
\hline 24 & 761 & 757 & 759 & 760 & 753 & 757 & & & & & & \\
\hline 25 & 761 & 755 & 758 & 757 & 752 & 754 & & & & & & \\
\hline 26 & 759 & 755 & 757 & 758 & 752 & 755 & & & & & & \\
\hline 27 & 760 & 757 & 759 & 761 & 756 & 759 & & & & & & \\
\hline 28 & 766 & 759 & 762 & 762 & 759 & 760 & & & & & & \\
\hline 29 & 768 & 763 & 765 & 761 & 755 & 758 & & & & & & \\
\hline 30 & 769 & 765 & 767 & 762 & 757 & 760 & & & & & & \\
\hline 31 & 770 & 760 & 765 & 762 & 759 & 761 & & & & & & \\
\hline Month & 770 & 750 & 760 & 769 & 752 & 762 & & & & & & \\
\hline
\end{tabular}


Table 35. Daily summary of total dissolved gas, in millimeters of mercury, for the Columbia River, left bank, near Dodson, Oregon, March - September 1996

[Max, maximum; Min, minimum; Month, monthly summary statistics; --, not available]

\begin{tabular}{|c|c|c|c|c|c|c|c|c|c|c|c|c|}
\hline \multirow{2}{*}{ Day } & \multicolumn{3}{|c|}{ March } & \multicolumn{3}{|c|}{ April } & \multicolumn{3}{|c|}{ May } & \multicolumn{3}{|c|}{ June } \\
\hline & Max & Min & Mean & Max & Min & Mean & Max & Min & Mean & Max & Min & Mean \\
\hline 1 & & & & -- & -- & -- & 942 & 912 & 927 & 1040 & 964 & 996 \\
\hline 2 & & & & 863 & 841 & 847 & 931 & 919 & 924 & 1040 & 961 & 990 \\
\hline 3 & & & & 870 & 860 & 864 & 972 & 914 & 929 & 992 & 954 & 975 \\
\hline 4 & & & & 875 & 858 & 866 & 952 & 910 & 924 & 977 & 948 & 954 \\
\hline 5 & & & & 882 & 867 & 872 & 955 & 922 & 934 & 980 & 949 & 960 \\
\hline 6 & & & & 895 & 865 & 878 & 968 & 918 & 934 & 1000 & 973 & 986 \\
\hline 7 & & & & 915 & 874 & 890 & 920 & 898 & 909 & 983 & 949 & 965 \\
\hline 8 & 949 & 918 & 930 & 893 & 865 & 875 & 921 & 899 & 912 & 983 & 956 & 974 \\
\hline 9 & 958 & 913 & 930 & 870 & 842 & 859 & 929 & 911 & 922 & 1010 & 976 & 988 \\
\hline 10 & 968 & 902 & 940 & 902 & 835 & 871 & 913 & 895 & 904 & 1020 & 986 & 995 \\
\hline 11 & 963 & 873 & 921 & 933 & 884 & 912 & 913 & 886 & 898 & 989 & 985 & 987 \\
\hline 12 & 908 & 873 & 889 & 945 & 917 & 931 & 919 & 886 & 896 & 992 & 979 & 987 \\
\hline 13 & 915 & 875 & 904 & 933 & 912 & 919 & 923 & 902 & 912 & 1020 & 956 & 974 \\
\hline 14 & 936 & 901 & 918 & 933 & 898 & 913 & 923 & 899 & 914 & 1030 & 946 & 976 \\
\hline 15 & 950 & 899 & 931 & 937 & 905 & 918 & 935 & 905 & 923 & 1050 & 948 & 974 \\
\hline 16 & 952 & 893 & 918 & 946 & 886 & 910 & 939 & 923 & 930 & 1050 & 946 & 993 \\
\hline 17 & 954 & 891 & 919 & 900 & 889 & 895 & 939 & 923 & 932 & 1050 & 949 & 974 \\
\hline 18 & 953 & 880 & 909 & 912 & 891 & 900 & 934 & 919 & 926 & 1030 & 959 & 979 \\
\hline 19 & 919 & 882 & 898 & 922 & 912 & 916 & 981 & 929 & 953 & 987 & 970 & 977 \\
\hline 20 & 923 & 873 & 894 & 924 & 895 & 911 & 1020 & 944 & 980 & 988 & 948 & 967 \\
\hline 21 & 919 & 879 & 898 & 929 & 903 & 917 & 1020 & 952 & 972 & 967 & 907 & 941 \\
\hline 22 & 910 & 881 & 891 & 925 & 901 & 914 & 981 & 926 & 947 & 932 & 895 & 909 \\
\hline 23 & 929 & 887 & 901 & 926 & 901 & 912 & 953 & 923 & 935 & 945 & 900 & 912 \\
\hline 24 & 929 & 883 & 900 & 918 & 901 & 911 & 1010 & 948 & 974 & 955 & 908 & 927 \\
\hline 25 & 923 & 883 & 900 & 942 & 913 & 933 & 1010 & 942 & 975 & 984 & 930 & 949 \\
\hline 26 & 932 & 886 & 914 & 950 & 934 & 941 & 980 & 938 & 959 & 1030 & 911 & 954 \\
\hline 27 & -- & -- & -- & 957 & 940 & 950 & 960 & 920 & 937 & 973 & 913 & 930 \\
\hline 28 & -- & -- & -- & 964 & 940 & 950 & 975 & 926 & 946 & 938 & 914 & 922 \\
\hline 29 & -- & -- & -- & 972 & 950 & 961 & 981 & 923 & 946 & 927 & 913 & 918 \\
\hline 30 & -- & -- & -- & 962 & 921 & 942 & 1000 & 941 & 964 & 930 & 914 & 923 \\
\hline 31 & -- & -- & -- & -- & -- & -- & 1010 & 946 & 974 & -- & -- & -- \\
\hline Month & -- & -- & -- & -- & -- & -- & 1020 & 886 & 936 & 1050 & 895 & 962 \\
\hline \multirow{2}{*}{ Day } & \multicolumn{3}{|c|}{ July } & \multicolumn{3}{|c|}{ August } & \multicolumn{3}{|c|}{ September } & & & \\
\hline & Max & Min & Mean & Max & Min & Mean & Max & Min & Mean & & & \\
\hline 1 & 932 & 892 & 915 & 884 & 852 & 867 & 891 & 822 & 841 & & & \\
\hline 2 & 901 & 871 & 892 & 880 & 855 & 866 & 830 & 821 & 825 & & & \\
\hline 3 & 885 & 871 & 880 & 888 & 865 & 874 & 825 & 814 & 819 & & & \\
\hline 4 & 898 & 877 & 888 & 902 & 857 & 875 & 825 & 813 & 816 & & & \\
\hline 5 & 903 & 887 & 897 & 902 & 851 & 873 & 819 & 806 & 813 & & & \\
\hline 6 & 918 & 889 & 909 & 884 & 859 & 869 & 813 & 796 & 805 & & & \\
\hline 7 & 913 & 904 & 908 & 905 & 867 & 889 & 818 & 802 & 810 & & & \\
\hline 8 & 919 & 893 & 911 & 903 & 886 & 898 & 802 & 777 & 786 & & & \\
\hline 9 & 899 & 858 & 880 & 911 & 894 & 900 & 782 & 773 & 778 & & & \\
\hline 10 & 888 & 859 & 876 & 910 & 893 & 901 & 781 & 769 & 775 & & & \\
\hline 11 & 896 & 887 & 890 & 907 & 875 & 890 & 778 & 767 & 775 & & & \\
\hline 12 & 915 & 890 & 908 & 897 & 865 & 878 & 778 & 770 & 774 & & & \\
\hline 13 & 917 & 884 & 904 & 890 & 868 & 876 & 776 & 768 & 772 & & & \\
\hline 14 & 903 & 886 & 898 & 898 & 845 & 868 & 774 & 766 & 770 & & & \\
\hline 15 & 915 & 875 & 897 & 883 & 847 & 858 & 773 & 764 & 770 & & & \\
\hline 16 & 891 & 846 & 870 & 891 & 847 & 860 & 774 & 766 & 769 & & & \\
\hline 17 & 871 & 846 & 857 & 904 & 861 & 880 & & & & & & \\
\hline 18 & 898 & 871 & 882 & 907 & 875 & 886 & & & & & & \\
\hline 19 & 908 & 877 & 892 & 904 & 858 & 877 & & & & & & \\
\hline 20 & 901 & 874 & 883 & 902 & 869 & 881 & & & & & & \\
\hline 21 & 910 & 881 & 891 & 902 & 876 & 887 & & & & & & \\
\hline 22 & 907 & 882 & 891 & 910 & 882 & 895 & & & & & & \\
\hline 23 & 908 & 888 & 897 & 909 & 879 & 891 & & & & & & \\
\hline 24 & 909 & 892 & 901 & 914 & 883 & 897 & & & & & & \\
\hline 25 & 906 & 884 & 894 & 920 & 877 & 895 & & & & & & \\
\hline 26 & 902 & 887 & 894 & 917 & 882 & 901 & & & & & & \\
\hline 27 & 905 & 885 & 895 & 920 & 852 & 886 & & & & & & \\
\hline 28 & 900 & 885 & 892 & 901 & 854 & 876 & & & & & & \\
\hline 29 & 909 & 876 & 892 & 890 & 866 & 878 & & & & & & \\
\hline 30 & 890 & 864 & 876 & 906 & 866 & 883 & & & & & & \\
\hline 31 & 908 & 864 & 878 & 902 & 854 & 877 & & & & & & \\
\hline Month & 932 & 846 & 892 & 920 & 845 & 882 & & & & & & \\
\hline
\end{tabular}


Table 36. Daily summary of total dissolved gas, in percent saturation, for the Columbia River, left bank, near Dodson, Oregon, March - September 1996

[Max, maximum; Min, minimum; Month, monthly summary statistics; --, not available]

\begin{tabular}{|c|c|c|c|c|c|c|c|c|c|c|c|c|}
\hline \multirow{2}{*}{ Day } & \multicolumn{3}{|c|}{ March } & \multicolumn{3}{|c|}{ April } & \multicolumn{3}{|c|}{ May } & \multicolumn{3}{|c|}{ June } \\
\hline & Max & Min & Mean & Max & Min & Mean & Max & Min & Mean & Max & Min & Mean \\
\hline 1 & & & & -- & -- & -- & 123 & 119 & 121 & 136 & 126 & 130 \\
\hline 2 & & & & 113 & 110 & 111 & 122 & 120 & 121 & 136 & 126 & 129 \\
\hline 3 & & & & 114 & 112 & 113 & 127 & 119 & 122 & 130 & 124 & 128 \\
\hline 4 & & & & 114 & 112 & 113 & 124 & 118 & 121 & 127 & 123 & 124 \\
\hline 5 & & & & 115 & 113 & 114 & 125 & 120 & 122 & 128 & 123 & 125 \\
\hline 6 & & & & 118 & 113 & 115 & 126 & 120 & 122 & 131 & 127 & 129 \\
\hline 7 & & & & 121 & 115 & 118 & 121 & 117 & 119 & 129 & 124 & 126 \\
\hline 8 & 124 & 119 & 121 & 118 & 114 & 115 & 120 & 118 & 119 & 128 & 125 & 127 \\
\hline 9 & 125 & 119 & 122 & 114 & 110 & 113 & 121 & 119 & 120 & 132 & 127 & 129 \\
\hline 10 & 127 & 118 & 123 & 118 & 110 & 114 & 119 & 116 & 118 & 133 & 128 & 130 \\
\hline 11 & 126 & 114 & 121 & 123 & 116 & 120 & 119 & 116 & 117 & 130 & 128 & 129 \\
\hline 12 & 119 & 114 & 116 & 124 & 120 & 123 & 121 & 116 & 117 & 130 & 128 & 129 \\
\hline 13 & 119 & 114 & 118 & 122 & 119 & 120 & 122 & 119 & 120 & 134 & 125 & 127 \\
\hline 14 & 122 & 117 & 120 & 123 & 118 & 120 & 122 & 119 & 121 & 135 & 123 & 127 \\
\hline 15 & 124 & 116 & 121 & 125 & 120 & 122 & 124 & 120 & 122 & 137 & 124 & 127 \\
\hline 16 & 123 & 115 & 119 & 127 & 118 & 122 & 125 & 122 & 123 & 138 & 124 & 130 \\
\hline 17 & 124 & 116 & 119 & 120 & 118 & 119 & 125 & 123 & 124 & 138 & 124 & 127 \\
\hline 18 & 124 & 115 & 119 & 120 & 118 & 118 & 124 & 121 & 123 & 134 & 125 & 127 \\
\hline 19 & 120 & 115 & 117 & 121 & 120 & 120 & 128 & 122 & 125 & 129 & 127 & 128 \\
\hline 20 & 120 & 114 & 117 & 121 & 118 & 119 & 134 & 123 & 129 & 130 & 125 & 127 \\
\hline 21 & 120 & 115 & 118 & 122 & 119 & 121 & 135 & 126 & 129 & 127 & 119 & 123 \\
\hline 22 & 121 & 116 & 118 & 122 & 119 & 120 & 129 & 121 & 124 & 122 & 117 & 119 \\
\hline 23 & 122 & 116 & 118 & 123 & 120 & 121 & 124 & 120 & 122 & 124 & 119 & 120 \\
\hline 24 & 122 & 115 & 118 & 121 & 118 & 120 & 132 & 123 & 127 & 126 & 120 & 122 \\
\hline 25 & 120 & 114 & 117 & 123 & 119 & 122 & 132 & 124 & 128 & 129 & 122 & 125 \\
\hline 26 & 122 & 117 & 120 & 124 & 121 & 123 & 129 & 123 & 126 & 136 & 121 & 126 \\
\hline 27 & -- & -- & -- & 124 & 122 & 123 & 126 & 120 & 123 & 128 & 120 & 122 \\
\hline 28 & -- & -- & -- & 126 & 122 & 124 & 128 & 121 & 124 & 122 & 119 & 120 \\
\hline 29 & -- & -- & -- & 127 & 123 & 125 & 128 & 121 & 124 & 120 & 119 & 120 \\
\hline 30 & -- & -- & -- & 125 & 120 & 123 & 131 & 123 & 126 & 122 & 119 & 121 \\
\hline 31 & -- & -- & -- & -- & -- & -- & 132 & 124 & 127 & -- & -- & -- \\
\hline Month & -- & -- & -- & -- & -- & -- & 135 & 116 & 123 & 138 & 117 & 126 \\
\hline \multirow{2}{*}{ Day } & \multicolumn{3}{|c|}{ July } & \multicolumn{3}{|c|}{ August } & \multicolumn{3}{|c|}{ September } & & & \\
\hline & Max & Min & Mean & Max & Min & Mean & Max & Min & Mean & & & \\
\hline 1 & 122 & 117 & 120 & 116 & 111 & 114 & 117 & 108 & 111 & & & \\
\hline 2 & 119 & 115 & 118 & 115 & 112 & 113 & 110 & 108 & 109 & & & \\
\hline 3 & 117 & 115 & 116 & 116 & 113 & 114 & 109 & 107 & 108 & & & \\
\hline 4 & 117 & 115 & 116 & 118 & 112 & 115 & 109 & 107 & 108 & & & \\
\hline 5 & 118 & 116 & 117 & 118 & 111 & 114 & 108 & 106 & 107 & & & \\
\hline 6 & 120 & 116 & 119 & 115 & 112 & 114 & 107 & 105 & 106 & & & \\
\hline 7 & 121 & 119 & 120 & 118 & 113 & 117 & 108 & 106 & 107 & & & \\
\hline 8 & 122 & 118 & 121 & 118 & 116 & 118 & 106 & 102 & 104 & & & \\
\hline 9 & 118 & 113 & 116 & 119 & 117 & 118 & 103 & 102 & 103 & & & \\
\hline 10 & 116 & 113 & 115 & 120 & 118 & 119 & 104 & 101 & 103 & & & \\
\hline 11 & 118 & 116 & 117 & 120 & 114 & 117 & 104 & 102 & 103 & & & \\
\hline 12 & 121 & 117 & 120 & 117 & 113 & 115 & 103 & 102 & 103 & & & \\
\hline 13 & 121 & 117 & 120 & 117 & 114 & 115 & 103 & 102 & 102 & & & \\
\hline 14 & 120 & 118 & 119 & 118 & 110 & 114 & 104 & 102 & 102 & & & \\
\hline 15 & 122 & 116 & 119 & 116 & 111 & 112 & 103 & 101 & 102 & & & \\
\hline 16 & 117 & 112 & 115 & 117 & 111 & 113 & 102 & 100 & 101 & & & \\
\hline 17 & 115 & 112 & 113 & 118 & 112 & 115 & & & & & & \\
\hline 18 & 118 & 115 & 116 & 118 & 114 & 116 & & & & & & \\
\hline 19 & 119 & 115 & 117 & 118 & 113 & 115 & & & & & & \\
\hline 20 & 118 & 115 & 116 & 118 & 114 & 116 & & & & & & \\
\hline 21 & 119 & 116 & 117 & 118 & 115 & 116 & & & & & & \\
\hline 22 & 119 & 116 & 117 & 119 & 116 & 118 & & & & & & \\
\hline 23 & 119 & 117 & 118 & 120 & 116 & 117 & & & & & & \\
\hline 24 & 120 & 118 & 119 & 121 & 117 & 119 & & & & & & \\
\hline 25 & 119 & 117 & 118 & 122 & 116 & 119 & & & & & & \\
\hline 26 & 119 & 117 & 118 & 121 & 117 & 119 & & & & & & \\
\hline 27 & 119 & 117 & 118 & 121 & 112 & 117 & & & & & & \\
\hline 28 & 118 & 116 & 117 & 119 & 112 & 115 & & & & & & \\
\hline 29 & 118 & 114 & 117 & 117 & 114 & 116 & & & & & & \\
\hline 30 & 116 & 112 & 114 & 119 & 114 & 116 & & & & & & \\
\hline 31 & 118 & 112 & 115 & 119 & 112 & 115 & & & & & & \\
\hline Month & 122 & 112 & 117 & 122 & 110 & 116 & & & & & & \\
\hline
\end{tabular}



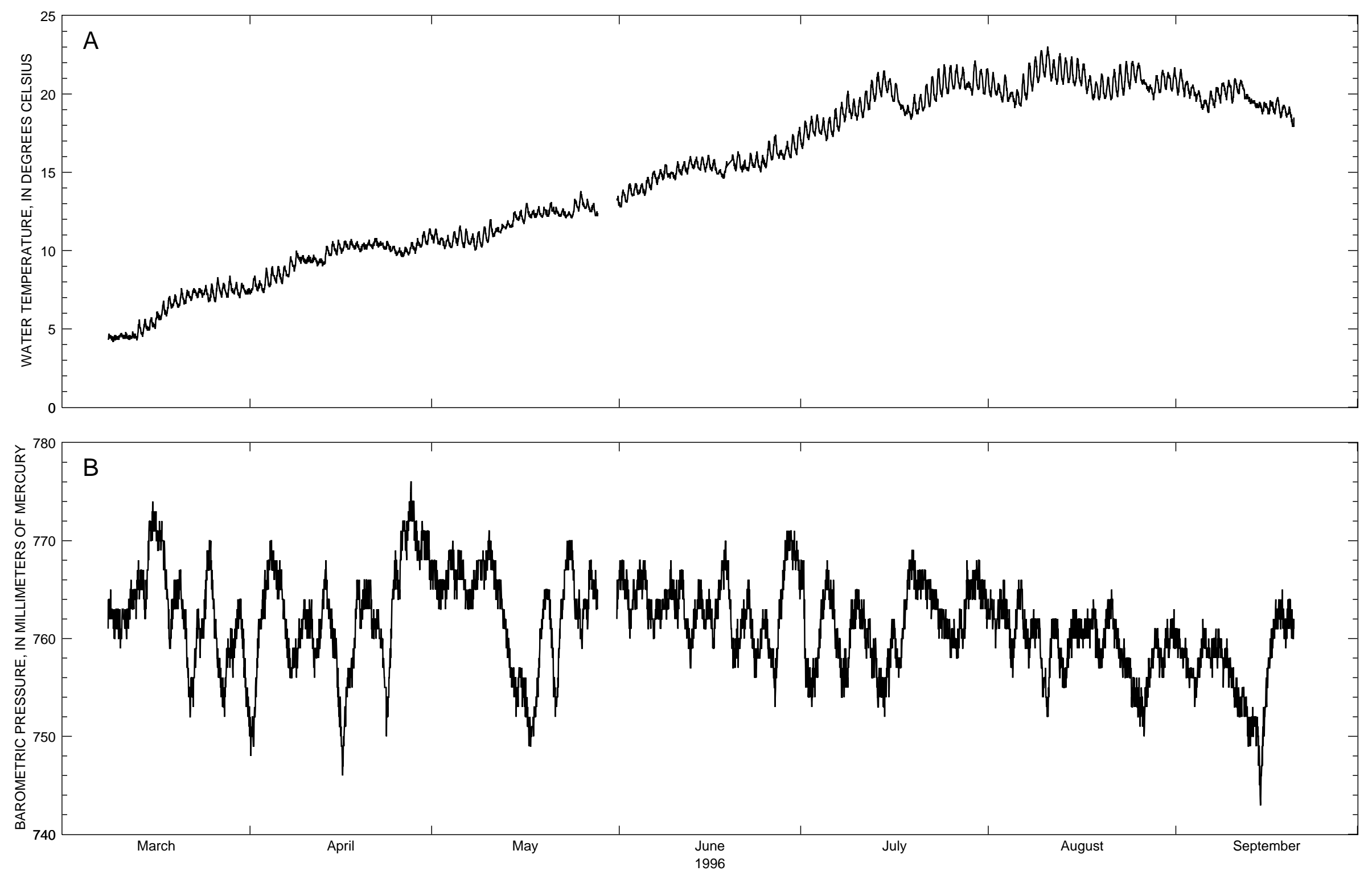

Figure 13. Hourly values of (A) water temperature, (B) barometric pressure, and (C, D) total dissolved gas at the Columbia River, right bank, near Washougal, Washington, March - September 1996. 

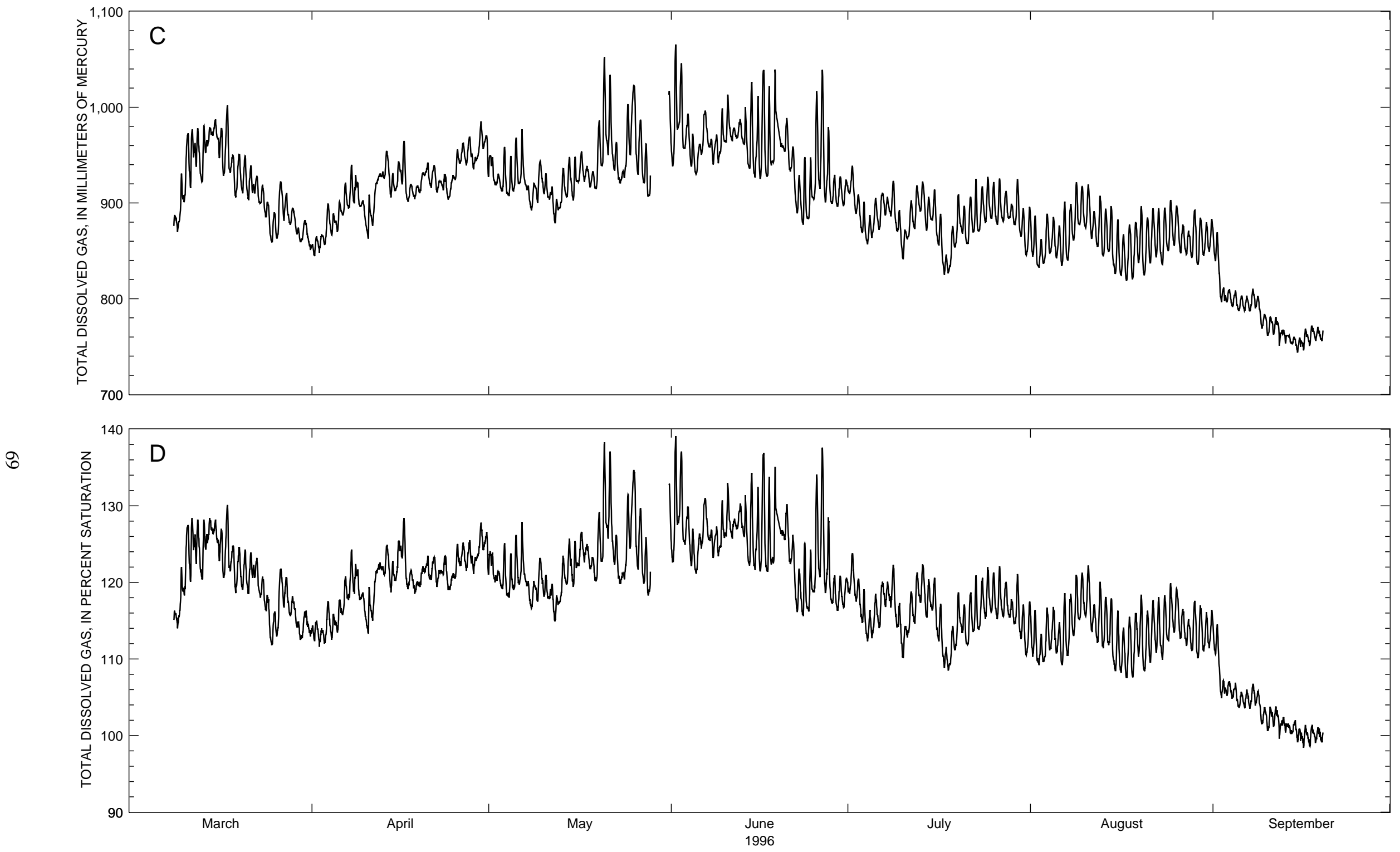

Figure 13. Hourly values of (A) water temperature, (B) barometric pressure, and (C, D) total dissolved gas at the Columbia River, right bank, near Washougal, Washington, March - September 1996. -Continued 
Table 37. Daily summary of water temperature for the Columbia River, right bank, near Washougal, Washington, March - September 1996

[Values reported in degrees Celsius; Max, maximum; Min, minimum; Month, monthly summary statistics; --, not available]

\begin{tabular}{|c|c|c|c|c|c|c|c|c|c|c|c|c|}
\hline \multirow{2}{*}{ Day } & \multicolumn{3}{|c|}{ March } & \multicolumn{3}{|c|}{ April } & \multicolumn{3}{|c|}{ May } & \multicolumn{3}{|c|}{ June } \\
\hline & Max & Min & Mean & Max & Min & Mean & Max & Min & Mean & Max & Min & Mean \\
\hline 1 & & & & 8.4 & 7.2 & 7.8 & 11.4 & 10.5 & 10.9 & 13.9 & 12.8 & 13.3 \\
\hline 2 & & & & 8.1 & 7.4 & 7.7 & 10.8 & 10.3 & 10.6 & 14.3 & 13.1 & 13.6 \\
\hline 3 & & & & 8.9 & 7.3 & 8.0 & 11.0 & 10.1 & 10.5 & 14.3 & 13.5 & 13.8 \\
\hline 4 & & & & 9.0 & 7.7 & 8.2 & 11.2 & 10.2 & 10.6 & 14.3 & 13.6 & 13.9 \\
\hline 5 & & & & 9.0 & 7.9 & 8.4 & 11.6 & 10.3 & 10.9 & 14.7 & 13.5 & 14.1 \\
\hline 6 & & & & 9.0 & 7.9 & 8.5 & 11.4 & 10.2 & 10.7 & 15.1 & 13.9 & 14.5 \\
\hline 7 & & & & 9.6 & 8.3 & 8.9 & 11.1 & 10.4 & 10.6 & 15.2 & 14.2 & 14.7 \\
\hline 8 & & & & 10.0 & 8.7 & 9.3 & 11.2 & 10.0 & 10.6 & 15.5 & 14.5 & 15.0 \\
\hline 9 & 4.6 & 4.2 & 4.4 & 9.7 & 9.2 & 9.5 & 11.5 & 10.2 & 10.8 & 15.0 & 14.6 & 14.9 \\
\hline 10 & 4.7 & 4.3 & 4.5 & 9.7 & 9.2 & 9.4 & 12.0 & 10.5 & 11.2 & 15.7 & 14.5 & 15.0 \\
\hline 11 & 4.8 & 4.3 & 4.5 & 9.7 & 9.2 & 9.4 & 11.5 & 10.9 & 11.2 & 15.7 & 14.8 & 15.2 \\
\hline 12 & 4.8 & 4.3 & 4.5 & 9.5 & 9.0 & 9.3 & 11.7 & 11.1 & 11.4 & 16.0 & 14.9 & 15.4 \\
\hline 13 & 5.6 & 4.3 & 4.8 & 10.3 & 9.1 & 9.7 & 11.9 & 11.4 & 11.6 & 16.0 & 15.2 & 15.6 \\
\hline 14 & 5.6 & 4.5 & 5.0 & 10.6 & 9.7 & 10.0 & 12.5 & 11.5 & 11.9 & 16.0 & 15.1 & 15.5 \\
\hline 15 & 5.7 & 4.9 & 5.2 & 10.7 & 9.7 & 10.2 & 12.6 & 11.9 & 12.2 & 16.1 & 15.0 & 15.5 \\
\hline 16 & 6.1 & 5.0 & 5.5 & 10.6 & 9.8 & 10.2 & 13.0 & 11.7 & 12.3 & 15.8 & 15.1 & 15.4 \\
\hline 17 & 6.8 & 5.6 & 6.1 & 10.7 & 10.1 & 10.3 & 12.6 & 12.1 & 12.3 & 15.3 & 14.7 & 15.0 \\
\hline 18 & 7.1 & 5.8 & 6.5 & 10.6 & 10.1 & 10.3 & 12.8 & 12.1 & 12.4 & 15.7 & 14.6 & -- \\
\hline 19 & 7.2 & 6.3 & 6.7 & 10.5 & 9.9 & 10.2 & 12.8 & 12.1 & 12.4 & 16.1 & -- & -- \\
\hline 20 & 7.6 & 6.4 & 6.9 & 10.7 & 10.1 & 10.3 & 13.1 & 12.2 & 12.6 & 16.3 & 15.1 & 15.7 \\
\hline 21 & 7.5 & 6.6 & 7.0 & 10.8 & 10.1 & 10.4 & 12.8 & 12.2 & 12.5 & 15.8 & 15.0 & 15.3 \\
\hline 22 & 7.6 & 6.8 & 7.2 & 10.6 & 10.2 & 10.4 & 12.7 & 12.2 & 12.4 & 16.2 & 15.1 & 15.6 \\
\hline 23 & 7.6 & 7.0 & 7.3 & 10.6 & 10.1 & 10.3 & 12.5 & 12.1 & 12.3 & 16.3 & 15.3 & 15.7 \\
\hline 24 & 7.8 & 7.0 & 7.3 & 10.3 & 9.9 & 10.1 & 13.3 & 12.1 & 12.6 & 16.1 & 15.0 & 15.5 \\
\hline 25 & 7.9 & 6.7 & 7.2 & 10.2 & 9.7 & 9.9 & 13.8 & 12.5 & 13.1 & 17.0 & 15.3 & 16.1 \\
\hline 26 & 8.3 & 6.7 & 7.4 & 10.2 & 9.6 & 9.9 & 13.3 & 12.7 & 12.9 & 17.4 & 15.8 & 16.5 \\
\hline 27 & 7.9 & 7.0 & 7.5 & 10.5 & 9.7 & 10.1 & 13.0 & 12.4 & 12.7 & 16.7 & 15.9 & 16.2 \\
\hline 28 & 8.4 & 7.0 & 7.6 & 10.8 & 9.8 & 10.3 & -- & -- & -- & 17.0 & 15.8 & 16.3 \\
\hline 29 & 8.0 & 7.2 & 7.5 & 11.2 & 10.2 & 10.7 & -- & -- & -- & 17.4 & 15.9 & 16.6 \\
\hline 30 & 7.9 & 7.0 & 7.4 & 11.4 & 10.4 & 10.9 & -- & -- & -- & 17.9 & 16.1 & 16.9 \\
\hline 31 & 7.6 & 7.2 & 7.4 & -- & -- & -- & -- & -- & -- & -- & -- & -- \\
\hline Month & -- & -- & -- & 11.4 & 7.2 & 9.6 & -- & -- & -- & 17.9 & -- & -- \\
\hline \multirow{2}{*}{ Day } & \multicolumn{3}{|c|}{ July } & \multicolumn{3}{|c|}{ August } & \multicolumn{3}{|c|}{ September } & & & \\
\hline & Max & Min & Mean & Max & Min & Mean & Max & Min & Mean & & & \\
\hline 1 & 18.3 & 16.5 & 17.3 & 21.4 & 19.8 & 20.6 & 21.6 & 19.9 & 20.8 & & & \\
\hline 2 & 18.6 & 17.1 & 17.8 & 20.7 & 19.9 & 20.2 & 21.0 & 19.8 & 20.5 & & & \\
\hline 3 & 18.7 & 17.3 & 17.9 & 21.2 & 19.5 & 20.3 & 20.6 & 19.7 & 20.2 & & & \\
\hline 4 & 18.5 & 17.4 & 17.8 & 20.4 & 19.5 & 19.9 & 20.2 & 19.5 & 19.9 & & & \\
\hline 5 & 18.5 & 17.0 & 17.8 & 20.3 & 19.1 & 19.8 & 19.9 & 19.0 & 19.4 & & & \\
\hline 6 & 19.1 & 17.3 & 18.1 & 21.3 & 19.2 & 20.0 & 20.2 & 18.8 & 19.5 & & & \\
\hline 7 & 19.5 & 17.6 & 18.4 & 22.0 & 19.6 & 20.7 & 20.4 & 19.1 & 19.8 & & & \\
\hline 8 & 20.2 & 18.2 & 19.1 & 22.4 & 20.2 & 21.3 & 20.7 & 19.4 & 20.2 & & & \\
\hline 9 & 19.7 & 18.4 & 19.0 & 22.8 & 20.7 & 21.7 & 20.9 & 19.6 & 20.3 & & & \\
\hline 10 & 19.8 & 18.3 & 19.0 & 23.0 & 21.1 & 21.9 & 21.0 & 19.4 & 20.3 & & & \\
\hline 11 & 20.2 & 18.5 & 19.3 & 22.5 & 20.8 & 21.6 & 20.9 & 20.0 & 20.5 & & & \\
\hline 12 & 20.9 & 19.0 & 19.9 & 22.6 & 20.6 & 21.5 & 20.6 & 19.7 & 20.0 & & & \\
\hline 13 & 21.4 & 19.5 & 20.4 & 22.3 & 20.6 & 21.5 & 19.8 & 19.4 & 19.5 & & & \\
\hline 14 & 21.5 & 19.8 & 20.6 & 22.4 & 20.7 & 21.4 & 19.5 & 19.1 & 19.3 & & & \\
\hline 15 & 21.0 & 20.1 & 20.6 & 22.3 & 20.5 & 21.3 & 19.5 & 18.7 & 19.1 & & & \\
\hline 16 & 20.7 & 19.5 & 20.0 & 21.9 & 20.5 & 21.2 & 19.6 & 18.7 & 19.2 & & & \\
\hline 17 & 20.0 & 18.9 & 19.3 & 21.2 & 20.0 & 20.7 & 19.8 & 18.7 & 19.3 & & & \\
\hline 18 & 19.3 & 18.6 & 18.9 & 20.8 & 19.6 & 20.2 & 19.3 & 18.5 & 19.0 & & & \\
\hline 19 & 20.0 & 18.4 & 19.0 & 21.3 & 19.6 & 20.4 & 19.2 & 18.5 & 18.8 & & & \\
\hline 20 & 20.0 & 18.7 & 19.3 & 21.1 & 19.7 & 20.3 & & & & & & \\
\hline 21 & 20.6 & 18.9 & 19.7 & 21.6 & 19.6 & 20.5 & & & & & & \\
\hline 22 & 21.1 & 19.2 & 20.1 & 21.9 & 19.8 & 20.8 & & & & & & \\
\hline 23 & 21.6 & 19.5 & 20.4 & 22.1 & 19.9 & 20.9 & & & & & & \\
\hline 24 & 21.9 & 19.8 & 20.7 & 22.1 & 20.3 & 21.2 & & & & & & \\
\hline 25 & 21.8 & 20.0 & 20.9 & 22.0 & 20.7 & 21.4 & & & & & & \\
\hline 26 & 21.9 & 20.3 & 21.0 & 21.8 & 20.6 & 20.9 & & & & & & \\
\hline 27 & 21.7 & 20.3 & 20.9 & 20.7 & 20.1 & 20.4 & & & & & & \\
\hline 28 & 20.9 & 20.3 & 20.6 & 21.2 & 19.6 & 20.4 & & & & & & \\
\hline 29 & 22.1 & 19.9 & 21.0 & 21.5 & 20.1 & 20.7 & & & & & & \\
\hline 30 & 21.6 & 20.3 & 20.9 & 21.4 & 20.3 & 20.9 & & & & & & \\
\hline 31 & 21.6 & 20.0 & 20.8 & 21.7 & 20.2 & 20.8 & & & & & & \\
\hline Month & 22.1 & 16.5 & 19.6 & 23.0 & 19.1 & 20.8 & & & & & & \\
\hline
\end{tabular}


Table 38. Daily summary of barometric pressure for the Columbia River, right bank, near Washougal, Washington, March - September 1996

[Values reported in millimeters of mercury; Max, maximum; Min, minimum; Month, monthly summary statistics; --, not available]

\begin{tabular}{|c|c|c|c|c|c|c|c|c|c|c|c|c|}
\hline \multirow{2}{*}{ Day } & \multicolumn{3}{|c|}{ March } & \multicolumn{3}{|c|}{ April } & \multicolumn{3}{|c|}{ May } & \multicolumn{3}{|c|}{ June } \\
\hline & Max & Min & Mean & Max & Min & Mean & Max & Min & Mean & Max & Min & Mean \\
\hline 1 & -- & -- & -- & 756 & 748 & 751 & 768 & 764 & 767 & 768 & 764 & 766 \\
\hline 2 & -- & -- & -- & 763 & 754 & 760 & 766 & 763 & 765 & 767 & 760 & 764 \\
\hline 3 & -- & -- & -- & 768 & 762 & 765 & 769 & 763 & 766 & 766 & 763 & 764 \\
\hline 4 & -- & -- & -- & 770 & 765 & 768 & 770 & 764 & 768 & 768 & 764 & 766 \\
\hline 5 & -- & -- & -- & 768 & 764 & 766 & 769 & 764 & 767 & 768 & 761 & 765 \\
\hline 6 & -- & -- & -- & 767 & 760 & 764 & 768 & 763 & 765 & 764 & 760 & 762 \\
\hline 7 & -- & -- & -- & 762 & 756 & 758 & 767 & 763 & 764 & 764 & 761 & 763 \\
\hline 8 & -- & -- & -- & 760 & 756 & 758 & 768 & 765 & 767 & 765 & 762 & 763 \\
\hline 9 & 763 & 760 & 762 & 764 & 759 & 761 & 769 & 765 & 767 & 768 & 763 & 765 \\
\hline 10 & 763 & 759 & 761 & 764 & 760 & 762 & 771 & 767 & 769 & 766 & 761 & 764 \\
\hline 11 & 764 & 760 & 762 & 764 & 756 & 760 & 769 & 763 & 766 & 767 & 761 & 765 \\
\hline 12 & 766 & 762 & 764 & 764 & 758 & 760 & 766 & 761 & 764 & 762 & 757 & 760 \\
\hline 13 & 768 & 762 & 765 & 768 & 762 & 765 & 763 & 756 & 760 & 764 & 759 & 762 \\
\hline 14 & 767 & 762 & 765 & 764 & 758 & 761 & 757 & 752 & 756 & 766 & 761 & 764 \\
\hline 15 & 774 & 767 & 770 & 761 & 750 & 756 & 757 & 753 & 756 & 765 & 761 & 763 \\
\hline 16 & 773 & 769 & 771 & 755 & 746 & 751 & 756 & 751 & 753 & 764 & 759 & 761 \\
\hline 17 & 772 & 766 & 769 & 758 & 754 & 756 & 753 & 749 & 751 & 768 & 762 & 765 \\
\hline 18 & 767 & 759 & 763 & 766 & 757 & 762 & 761 & 752 & 755 & 770 & 765 & 768 \\
\hline 19 & 766 & 761 & 764 & 766 & 761 & 763 & 765 & 760 & 763 & 768 & 757 & 762 \\
\hline 20 & 767 & 762 & 765 & 766 & 763 & 765 & 765 & 758 & 763 & 762 & 757 & 759 \\
\hline 21 & 763 & 754 & 759 & 764 & 760 & 761 & 759 & 752 & 755 & 764 & 760 & 762 \\
\hline 22 & 759 & 752 & 755 & 763 & 757 & 760 & 767 & 758 & 763 & 766 & 759 & 763 \\
\hline 23 & 763 & 757 & 761 & 758 & 750 & 755 & 770 & 767 & 768 & 762 & 756 & 758 \\
\hline 24 & 769 & 760 & 764 & 768 & 756 & 762 & 770 & 762 & 766 & 763 & 758 & 760 \\
\hline 25 & 770 & 763 & 767 & 771 & 764 & 766 & 765 & 759 & 762 & 763 & 758 & 760 \\
\hline 26 & 764 & 756 & 759 & 772 & 768 & 771 & 767 & 761 & 763 & 759 & 753 & 757 \\
\hline 27 & 758 & 752 & 755 & 776 & 770 & 773 & 768 & 764 & 766 & 765 & 756 & 762 \\
\hline 28 & 761 & 756 & 759 & 774 & 768 & 770 & -- & -- & -- & 771 & 764 & 768 \\
\hline 29 & 764 & 756 & 760 & 772 & 767 & 770 & -- & -- & -- & 771 & 767 & 770 \\
\hline 30 & 764 & 756 & 762 & 771 & 765 & 769 & -- & -- & -- & 770 & 764 & 767 \\
\hline 31 & 758 & 750 & 754 & -- & -- & -- & -- & -- & -- & -- & -- & -- \\
\hline Month & -- & -- & -- & 776 & 746 & 762 & -- & -- & -- & 771 & 753 & 763 \\
\hline \multirow{2}{*}{ Day } & \multicolumn{3}{|c|}{ July } & \multicolumn{3}{|c|}{ August } & \multicolumn{3}{|c|}{ September } & & & \\
\hline & Max & Min & Mean & Max & Min & Mean & Max & Min & Mean & & & \\
\hline 1 & 768 & 755 & 763 & 765 & 761 & 763 & 761 & 758 & 759 & & & \\
\hline 2 & 758 & 753 & 755 & 765 & 759 & 762 & 760 & 756 & 758 & & & \\
\hline 3 & 759 & 754 & 757 & 766 & 761 & 763 & 758 & 755 & 756 & & & \\
\hline 4 & 765 & 758 & 762 & 764 & 757 & 760 & 758 & 753 & 756 & & & \\
\hline 5 & 768 & 763 & 765 & 765 & 756 & 761 & 762 & 757 & 760 & & & \\
\hline 6 & 766 & 758 & 762 & 766 & 760 & 763 & 760 & 756 & 758 & & & \\
\hline 7 & 761 & 754 & 758 & 763 & 757 & 760 & 761 & 756 & 759 & & & \\
\hline 8 & 759 & 754 & 757 & 763 & 759 & 761 & 761 & 755 & 759 & & & \\
\hline 9 & 764 & 758 & 762 & 762 & 754 & 760 & 760 & 755 & 758 & & & \\
\hline 10 & 765 & 761 & 763 & 757 & 752 & 755 & 758 & 753 & 755 & & & \\
\hline 11 & 764 & 758 & 761 & 762 & 754 & 760 & 756 & 752 & 753 & & & \\
\hline 12 & 762 & 756 & 759 & 762 & 756 & 760 & 755 & 749 & 753 & & & \\
\hline 13 & 760 & 753 & 757 & 759 & 755 & 757 & 753 & 749 & 751 & & & \\
\hline 14 & 757 & 752 & 755 & 763 & 757 & 760 & 752 & 743 & 749 & & & \\
\hline 15 & 761 & 754 & 757 & 763 & 759 & 761 & 756 & 743 & 750 & & & \\
\hline 16 & 762 & 757 & 760 & 762 & 759 & 761 & 761 & 753 & 758 & & & \\
\hline 17 & 761 & 756 & 758 & 763 & 759 & 761 & 764 & 759 & 762 & & & \\
\hline 18 & 768 & 761 & 764 & 764 & 758 & 762 & 765 & 760 & 762 & & & \\
\hline 19 & 769 & 765 & 767 & 761 & 757 & 758 & 764 & 759 & 762 & & & \\
\hline 20 & 767 & 764 & 765 & 764 & 759 & 762 & & & & & & \\
\hline 21 & 767 & 763 & 766 & 765 & 759 & 762 & & & & & & \\
\hline 22 & 766 & 762 & 765 & 762 & 757 & 759 & & & & & & \\
\hline 23 & 765 & 760 & 763 & 760 & 756 & 758 & & & & & & \\
\hline 24 & 763 & 760 & 762 & 758 & 753 & 756 & & & & & & \\
\hline 25 & 762 & 758 & 760 & 756 & 752 & 754 & & & & & & \\
\hline 26 & 762 & 758 & 760 & 755 & 750 & 753 & & & & & & \\
\hline 27 & 763 & 758 & 761 & 758 & 753 & 756 & & & & & & \\
\hline 28 & 768 & 760 & 764 & 761 & 757 & 759 & & & & & & \\
\hline 29 & 767 & 764 & 765 & 761 & 756 & 758 & & & & & & \\
\hline 30 & 768 & 763 & 766 & 762 & 757 & 760 & & & & & & \\
\hline 31 & 766 & 761 & 764 & 763 & 759 & 761 & & & & & & \\
\hline Month & 769 & 752 & 761 & 766 & 750 & 760 & & & & & & \\
\hline
\end{tabular}


Table 39. Daily summary of total dissolved gas, in millimeters of mercury, for the Columbia River, right bank, near Washougal, Washington, March - September 1996

[Max, maximum; Min, minimum; Month, monthly summary statistics; --, not available]

\begin{tabular}{|c|c|c|c|c|c|c|c|c|c|c|c|c|}
\hline \multirow{2}{*}{ Day } & \multicolumn{3}{|c|}{ March } & \multicolumn{3}{|c|}{ April } & \multicolumn{3}{|c|}{ May } & \multicolumn{3}{|c|}{ June } \\
\hline & Max & Min & Mean & Max & Min & Mean & Max & Min & Mean & Max & Min & Mean \\
\hline 1 & -- & -- & -- & 865 & 844 & 855 & 949 & 922 & 940 & 1070 & 938 & 986 \\
\hline 2 & -- & -- & -- & 867 & 848 & 860 & 931 & 917 & 923 & 1050 & 962 & 999 \\
\hline 3 & -- & -- & -- & 899 & 857 & 877 & 958 & 912 & 928 & 993 & 957 & 971 \\
\hline 4 & -- & -- & -- & 889 & 866 & 878 & 948 & 907 & 920 & 975 & 937 & 953 \\
\hline 5 & -- & -- & -- & 901 & 870 & 886 & 968 & 912 & 933 & 961 & 930 & 945 \\
\hline 6 & -- & -- & -- & 921 & 887 & 901 & 977 & 920 & 936 & 996 & 950 & 975 \\
\hline 7 & -- & -- & -- & 940 & 894 & 912 & 928 & 901 & 916 & 979 & 949 & 964 \\
\hline 8 & -- & -- & -- & 929 & 899 & 913 & 920 & 893 & 907 & 971 & 940 & 956 \\
\hline 9 & 931 & 870 & 892 & 902 & 885 & 897 & 944 & 900 & 923 & 999 & 941 & 966 \\
\hline 10 & 973 & 901 & 931 & 909 & 863 & 881 & 931 & 909 & 919 & 1010 & 963 & 981 \\
\hline 11 & 977 & 915 & 950 & 922 & 876 & 897 & 917 & 893 & 908 & 979 & 964 & 972 \\
\hline 12 & 978 & 934 & 957 & 932 & 920 & 927 & 904 & 879 & 892 & 987 & 968 & 976 \\
\hline 13 & 981 & 922 & 946 & 954 & 926 & 939 & 936 & 895 & 914 & 1000 & 943 & 969 \\
\hline 14 & 978 & 955 & 969 & 939 & 906 & 920 & 948 & 906 & 923 & 1030 & 936 & 966 \\
\hline 15 & 987 & 966 & 976 & 943 & 912 & 926 & 948 & 902 & 922 & 1010 & 927 & 956 \\
\hline 16 & 978 & 928 & 959 & 964 & 910 & 937 & 953 & 922 & 935 & 1040 & 926 & 976 \\
\hline 17 & 1000 & 929 & 961 & 919 & 902 & 910 & 939 & 922 & 931 & 1020 & 928 & 954 \\
\hline 18 & 950 & 908 & 935 & 918 & 904 & 911 & 933 & 916 & 924 & 1040 & -- & -- \\
\hline 19 & 951 & 906 & 930 & 932 & 910 & 921 & 986 & 914 & 945 & -- & -- & -- \\
\hline 20 & 949 & 910 & 927 & 942 & 920 & 932 & 1050 & 939 & 980 & 989 & 941 & 965 \\
\hline 21 & 939 & 903 & 919 & 939 & 916 & 929 & 1030 & 950 & 980 & 959 & 916 & 941 \\
\hline 22 & 928 & 901 & 917 & 924 & 911 & 919 & 963 & 928 & 946 & 929 & 889 & 906 \\
\hline 23 & 918 & 891 & 905 & 930 & 908 & 920 & 933 & 921 & 927 & 947 & 877 & 903 \\
\hline 24 & 901 & 866 & 887 & 929 & 903 & 914 & 1000 & 926 & 961 & 947 & 883 & 904 \\
\hline 25 & 890 & 859 & 874 & 956 & 924 & 937 & 1020 & 950 & 995 & 1020 & 904 & 945 \\
\hline 26 & 922 & 863 & 897 & 963 & 941 & 951 & 987 & 930 & 958 & 1040 & 915 & 963 \\
\hline 27 & 910 & 881 & 896 & 969 & 939 & 954 & 962 & 909 & 935 & 979 & 901 & 929 \\
\hline 28 & 894 & 877 & 886 & 951 & 935 & 945 & -- & -- & -- & 929 & 899 & 910 \\
\hline 29 & 888 & 859 & 872 & 985 & 947 & 964 & -- & -- & -- & 928 & 896 & 910 \\
\hline 30 & 882 & 859 & 870 & 970 & 927 & 958 & -- & -- & -- & 924 & 897 & 911 \\
\hline 31 & 879 & 851 & 861 & -- & -- & -- & -- & -- & -- & -- & -- & -- \\
\hline Month & -- & -- & -- & 985 & 844 & 916 & -- & -- & -- & -- & -- & -- \\
\hline \multirow{2}{*}{ Day } & \multicolumn{3}{|c|}{ July } & \multicolumn{3}{|c|}{ August } & \multicolumn{3}{|c|}{ September } & & & \\
\hline & Max & Min & Mean & Max & Min & Mean & Max & Min & Mean & & & \\
\hline 1 & 939 & 910 & 921 & 887 & 844 & 863 & 872 & 830 & 852 & & & \\
\hline 2 & 913 & 889 & 899 & 862 & 832 & 844 & 830 & 796 & 808 & & & \\
\hline 3 & 901 & 868 & 881 & 889 & 837 & 857 & 810 & 797 & 803 & & & \\
\hline 4 & 887 & 857 & 870 & 885 & 847 & 862 & 808 & 792 & 799 & & & \\
\hline 5 & 905 & 863 & 884 & 880 & 842 & 859 & 803 & 787 & 794 & & & \\
\hline 6 & 911 & 872 & 890 & 901 & 835 & 862 & 803 & 787 & 795 & & & \\
\hline 7 & 906 & 887 & 897 & 899 & 840 & 865 & 810 & 787 & 797 & & & \\
\hline 8 & 923 & 886 & 901 & 922 & 861 & 886 & 803 & 786 & 796 & & & \\
\hline 9 & 892 & 867 & 879 & 917 & 877 & 893 & 786 & 769 & 778 & & & \\
\hline 10 & 872 & 841 & 859 & 919 & 874 & 892 & 781 & 761 & 772 & & & \\
\hline 11 & 903 & 862 & 880 & 904 & 862 & 879 & 781 & 763 & 772 & & & \\
\hline 12 & 918 & 873 & 894 & 907 & 854 & 876 & 775 & 751 & 764 & & & \\
\hline 13 & 922 & 891 & 905 & 893 & 845 & 867 & 767 & 753 & 761 & & & \\
\hline 14 & 906 & 871 & 889 & 896 & 842 & 866 & 760 & 752 & 756 & & & \\
\hline 15 & 914 & 877 & 892 & 883 & 826 & 847 & 759 & 744 & 752 & & & \\
\hline 16 & 889 & 849 & 868 & 867 & 824 & 843 & 768 & 746 & 758 & & & \\
\hline 17 & 849 & 825 & 836 & 877 & 819 & 844 & 771 & 751 & 762 & & & \\
\hline 18 & 876 & 826 & 849 & 879 & 821 & 848 & 771 & 756 & 763 & & & \\
\hline 19 & 908 & 854 & 876 & 896 & 837 & 864 & & & & & & \\
\hline 20 & 897 & 865 & 879 & 885 & 825 & 850 & & & & & & \\
\hline 21 & 906 & 857 & 877 & 894 & 837 & 864 & & & & & & \\
\hline 22 & 925 & 870 & 888 & 894 & 843 & 865 & & & & & & \\
\hline 23 & 917 & 870 & 889 & 894 & 836 & 861 & & & & & & \\
\hline 24 & 927 & 879 & 899 & 903 & 850 & 872 & & & & & & \\
\hline 25 & 922 & 884 & 899 & 897 & 856 & 876 & & & & & & \\
\hline 26 & 925 & 877 & 896 & 889 & 847 & 865 & & & & & & \\
\hline 27 & 912 & 881 & 894 & 875 & 846 & 859 & & & & & & \\
\hline 28 & 903 & 884 & 890 & 892 & 843 & 864 & & & & & & \\
\hline 29 & 925 & 877 & 894 & 885 & 835 & 859 & & & & & & \\
\hline 30 & 899 & 865 & 878 & 880 & 845 & 861 & & & & & & \\
\hline 31 & 896 & 845 & 865 & 883 & 849 & 865 & & & & & & \\
\hline Month & 939 & 825 & 884 & 922 & 819 & 864 & & & & & & \\
\hline
\end{tabular}


Table 40. Daily summary of total dissolved gas, in percent saturation, for the Columbia River, right bank, near Washougal, Washington, March - September 1996

[Max, maximum; Min, minimum; Month, monthly summary statistics; --, not available]

\begin{tabular}{|c|c|c|c|c|c|c|c|c|c|c|c|c|}
\hline \multirow{2}{*}{ Day } & \multicolumn{3}{|c|}{ March } & \multicolumn{3}{|c|}{ April } & \multicolumn{3}{|c|}{ May } & \multicolumn{3}{|c|}{ June } \\
\hline & Max & Min & Mean & Max & Min & Mean & Max & Min & Mean & Max & Min & Mean \\
\hline 1 & -- & -- & -- & 115 & 112 & 114 & 124 & 120 & 123 & 139 & 123 & 129 \\
\hline 2 & -- & -- & -- & 114 & 112 & 113 & 121 & 120 & 121 & 137 & 126 & 131 \\
\hline 3 & -- & -- & -- & 117 & 112 & 115 & 125 & 119 & 121 & 130 & 125 & 127 \\
\hline 4 & -- & -- & -- & 116 & 112 & 114 & 124 & 118 & 120 & 127 & 122 & 124 \\
\hline 5 & -- & -- & -- & 118 & 113 & 116 & 126 & 119 & 122 & 126 & 121 & 124 \\
\hline 6 & -- & -- & -- & 121 & 116 & 118 & 128 & 120 & 122 & 131 & 124 & 128 \\
\hline 7 & -- & -- & -- & 124 & 118 & 120 & 121 & 118 & 120 & 128 & 125 & 126 \\
\hline 8 & -- & -- & -- & 122 & 118 & 120 & 120 & 117 & 118 & 127 & 123 & 125 \\
\hline 9 & 122 & 114 & 117 & 119 & 116 & 118 & 123 & 117 & 120 & 131 & 123 & 126 \\
\hline 10 & 127 & 118 & 122 & 119 & 113 & 116 & 121 & 118 & 120 & 133 & 126 & 128 \\
\hline 11 & 128 & 120 & 125 & 122 & 115 & 118 & 120 & 117 & 119 & 128 & 126 & 127 \\
\hline 12 & 128 & 122 & 125 & 123 & 121 & 122 & 118 & 115 & 117 & 130 & 127 & 128 \\
\hline 13 & 128 & 120 & 124 & 125 & 121 & 123 & 123 & 117 & 120 & 131 & 123 & 127 \\
\hline 14 & 128 & 124 & 127 & 123 & 119 & 121 & 126 & 120 & 122 & 134 & 122 & 126 \\
\hline 15 & 128 & 125 & 127 & 125 & 120 & 123 & 125 & 119 & 122 & 132 & 122 & 125 \\
\hline 16 & 127 & 121 & 124 & 128 & 120 & 125 & 127 & 122 & 124 & 137 & 121 & 128 \\
\hline 17 & 130 & 121 & 125 & 122 & 119 & 120 & 125 & 123 & 124 & 134 & 121 & 125 \\
\hline 18 & 125 & 119 & 123 & 121 & 118 & 120 & 123 & 121 & 122 & 135 & -- & -- \\
\hline 19 & 125 & 119 & 122 & 122 & 119 & 121 & 129 & 120 & 124 & -- & -- & -- \\
\hline 20 & 124 & 119 & 121 & 123 & 120 & 122 & 138 & 123 & 128 & 130 & 124 & 127 \\
\hline 21 & 124 & 118 & 121 & 123 & 120 & 122 & 137 & 125 & 130 & 126 & 120 & 124 \\
\hline 22 & 123 & 119 & 121 & 122 & 119 & 121 & 126 & 121 & 124 & 122 & 116 & 119 \\
\hline 23 & 121 & 117 & 119 & 123 & 120 & 122 & 121 & 120 & 121 & 125 & 116 & 119 \\
\hline 24 & 118 & 113 & 116 & 121 & 119 & 120 & 131 & 120 & 125 & 124 & 116 & 119 \\
\hline 25 & 116 & 112 & 114 & 125 & 120 & 122 & 135 & 124 & 131 & 134 & 119 & 124 \\
\hline 26 & 122 & 113 & 118 & 125 & 122 & 123 & 130 & 122 & 125 & 138 & 121 & 127 \\
\hline 27 & 121 & 117 & 119 & 125 & 121 & 123 & 126 & 119 & 122 & 128 & 119 & 122 \\
\hline 28 & 118 & 116 & 117 & 123 & 121 & 123 & -- & -- & -- & 121 & 117 & 119 \\
\hline 29 & 117 & 112 & 115 & 128 & 123 & 125 & -- & -- & -- & 121 & 116 & 118 \\
\hline 30 & 116 & 112 & 114 & 127 & 121 & 125 & -- & -- & -- & 121 & 117 & 119 \\
\hline 31 & 116 & 113 & 114 & -- & -- & -- & -- & -- & -- & -- & -- & -- \\
\hline Month & -- & -- & -- & 128 & 112 & 120 & -- & -- & -- & -- & -- & -- \\
\hline \multirow{2}{*}{ Day } & \multicolumn{3}{|c|}{ July } & \multicolumn{3}{|c|}{ August } & \multicolumn{3}{|c|}{ September } & & & \\
\hline & Max & Min & Mean & Max & Min & Mean & Max & Min & Mean & & & \\
\hline 1 & 124 & 118 & 121 & 116 & 110 & 113 & 115 & 109 & 112 & & & \\
\hline 2 & 121 & 117 & 119 & 113 & 109 & 111 & 109 & 105 & 107 & & & \\
\hline 3 & 119 & 114 & 116 & 117 & 110 & 112 & 107 & 105 & 106 & & & \\
\hline 4 & 116 & 112 & 114 & 117 & 111 & 113 & 107 & 105 & 106 & & & \\
\hline 5 & 118 & 113 & 116 & 116 & 111 & 113 & 106 & 104 & 105 & & & \\
\hline 6 & 120 & 114 & 117 & 119 & 109 & 113 & 106 & 104 & 105 & & & \\
\hline 7 & 120 & 117 & 118 & 118 & 110 & 114 & 107 & 103 & 105 & & & \\
\hline 8 & 122 & 117 & 119 & 121 & 113 & 116 & 106 & 104 & 105 & & & \\
\hline 9 & 117 & 113 & 115 & 121 & 115 & 118 & 104 & 101 & 103 & & & \\
\hline 10 & 114 & 110 & 113 & 122 & 116 & 118 & 104 & 101 & 102 & & & \\
\hline 11 & 119 & 113 & 116 & 120 & 113 & 116 & 104 & 101 & 102 & & & \\
\hline 12 & 121 & 115 & 118 & 120 & 112 & 115 & 103 & 100 & 102 & & & \\
\hline 13 & 122 & 117 & 120 & 118 & 111 & 115 & 102 & 100 & 101 & & & \\
\hline 14 & 120 & 115 & 118 & 118 & 110 & 114 & 102 & 100 & 101 & & & \\
\hline 15 & 121 & 115 & 118 & 116 & 108 & 111 & 102 & 99 & 100 & & & \\
\hline 16 & 117 & 112 & 114 & 114 & 108 & 111 & 101 & 98 & 100 & & & \\
\hline 17 & 112 & 109 & 110 & 115 & 107 & 111 & 101 & 99 & 100 & & & \\
\hline 18 & 114 & 108 & 111 & 116 & 108 & 111 & 101 & 99 & 100 & & & \\
\hline 19 & 119 & 111 & 114 & 118 & 110 & 114 & & & & & & \\
\hline 20 & 117 & 113 & 115 & 116 & 108 & 112 & & & & & & \\
\hline 21 & 119 & 112 & 115 & 118 & 109 & 113 & & & & & & \\
\hline 22 & 121 & 114 & 116 & 118 & 111 & 114 & & & & & & \\
\hline 23 & 121 & 114 & 117 & 118 & 110 & 114 & & & & & & \\
\hline 24 & 122 & 115 & 118 & 120 & 112 & 115 & & & & & & \\
\hline 25 & 121 & 116 & 118 & 119 & 113 & 116 & & & & & & \\
\hline 26 & 122 & 115 & 118 & 118 & 113 & 115 & & & & & & \\
\hline 27 & 120 & 116 & 118 & 116 & 112 & 114 & & & & & & \\
\hline 28 & 119 & 115 & 117 & 118 & 111 & 114 & & & & & & \\
\hline 29 & 121 & 115 & 117 & 117 & 110 & 113 & & & & & & \\
\hline 30 & 117 & 113 & 115 & 116 & 111 & 113 & & & & & & \\
\hline 31 & 117 & 110 & 113 & 116 & 111 & 114 & & & & & & \\
\hline Month & 124 & 108 & 116 & 122 & 107 & 114 & & & & & & \\
\hline
\end{tabular}



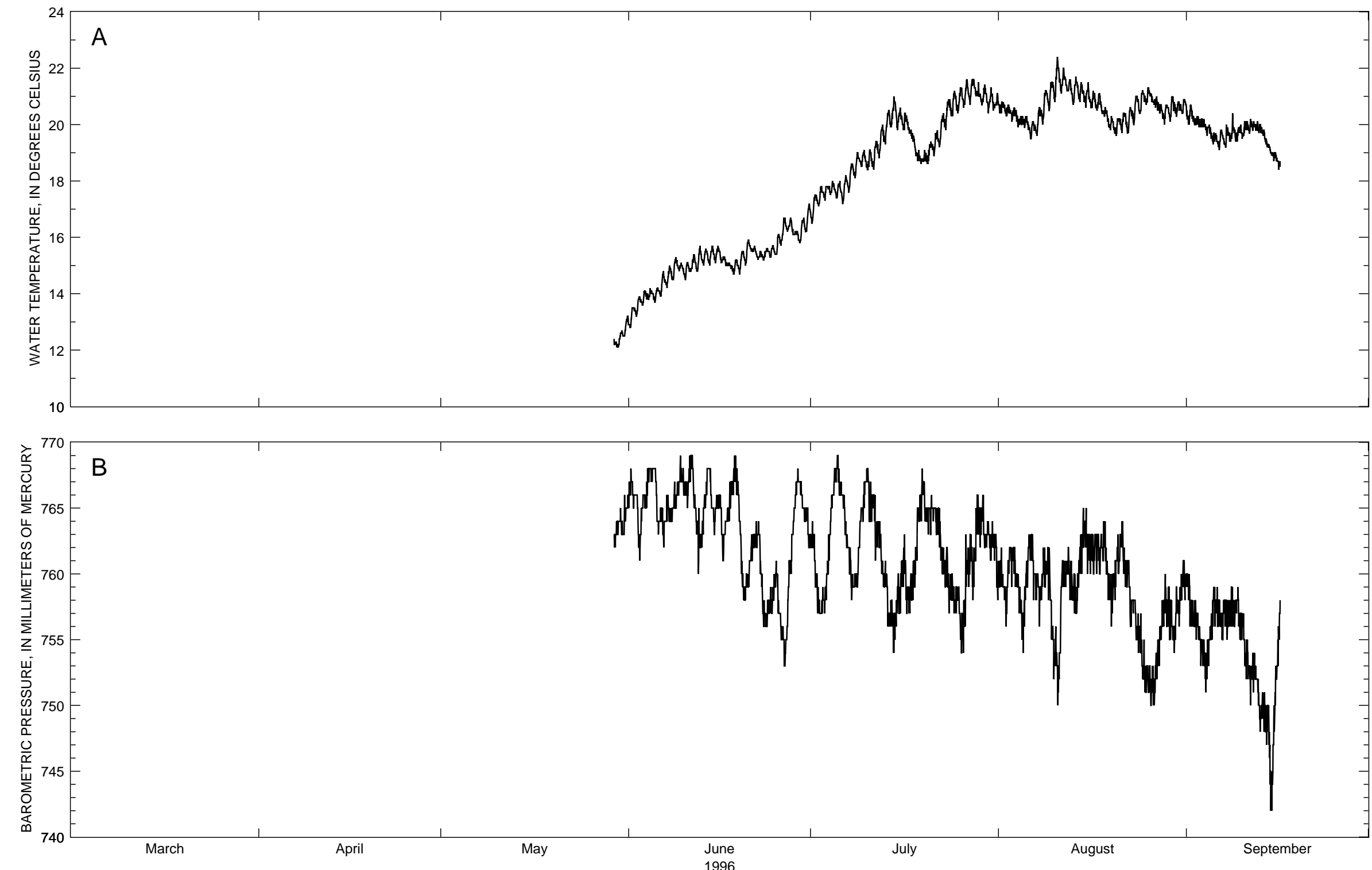

Figure 14. Hourly values of (A) water temperature, (B) barometric pressure, and (C, D) total dissolved gas at the Columbia River, right bank, near Kalama, Washington, May - September 1996. 

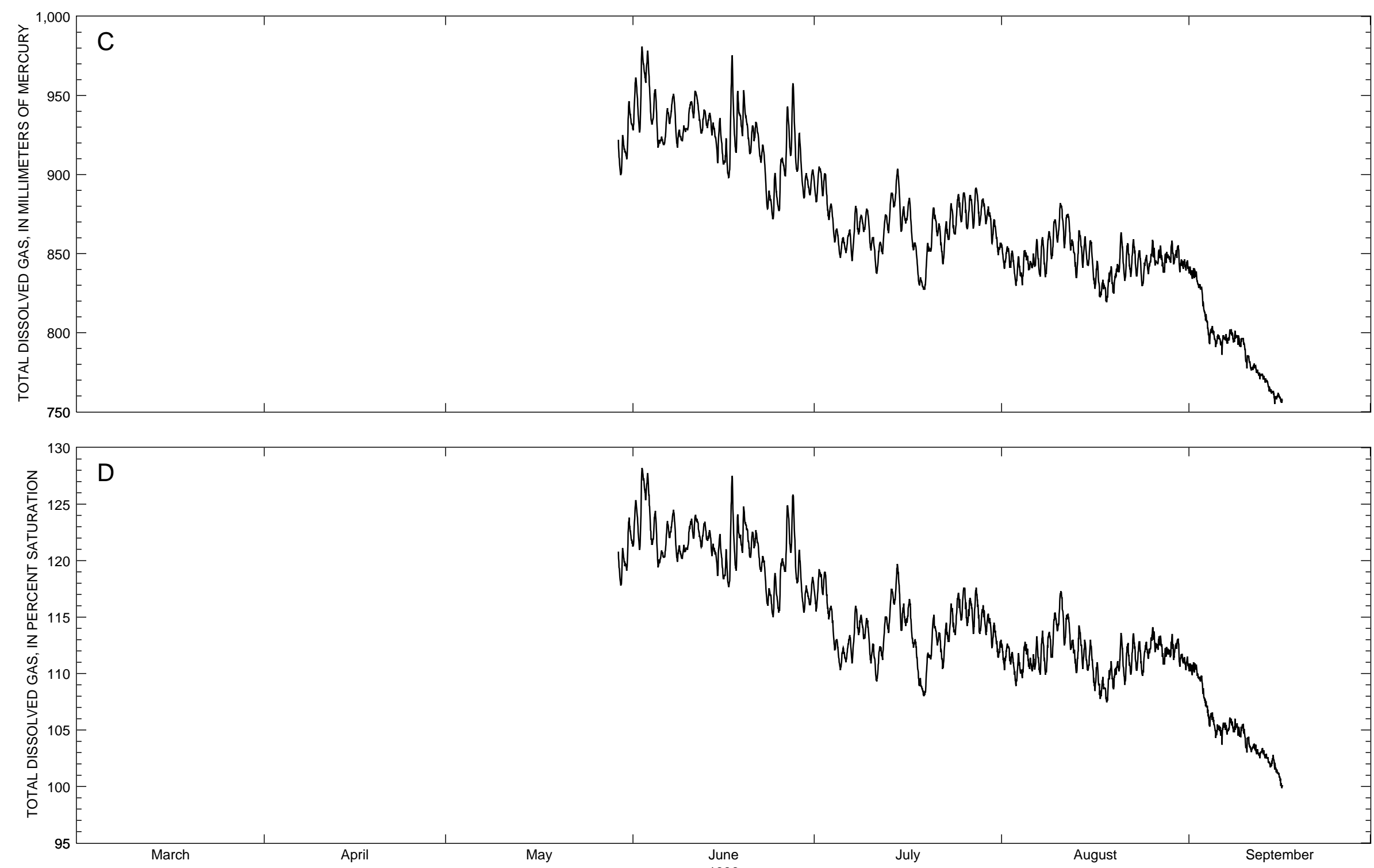

Figure 14. Hourly values of (A) water temperature, (B) barometric pressure, and (C, D) total dissolved gas at the Columbia River, right bank, near Kalama, Washington, May - September 1996.-continued 
Table 41. Daily summary of water temperature for the Columbia River, right bank, near Kalama, Washington, April - September 1996

[Values reported in degrees Celsius; Max, maximum; Min, minimum; Month, monthly summary statistics; --, not available]

\begin{tabular}{|c|c|c|c|c|c|c|c|c|c|c|c|c|}
\hline \multirow{2}{*}{ Day } & \multicolumn{3}{|c|}{ April } & \multicolumn{3}{|c|}{ May } & \multicolumn{3}{|c|}{ June } & \multicolumn{3}{|c|}{ July } \\
\hline & Max & Min & Mean & Max & Min & Mean & Max & Min & Mean & $\operatorname{Max}$ & Min & Mean \\
\hline 1 & -- & -- & -- & -- & -- & -- & 13.5 & 12.8 & 13.2 & 17.5 & 16.5 & 17.1 \\
\hline 2 & -- & -- & -- & -- & -- & -- & 13.9 & 13.2 & 13.6 & 17.8 & 17.1 & 17.4 \\
\hline 3 & -- & -- & -- & -- & -- & -- & 14.1 & 13.6 & 13.8 & 17.8 & 17.3 & 17.6 \\
\hline 4 & -- & -- & -- & -- & -- & -- & 14.2 & 13.8 & 14.0 & 18.0 & 17.5 & 17.7 \\
\hline 5 & -- & -- & -- & -- & -- & -- & 14.2 & 13.7 & 14.0 & 18.0 & 17.4 & 17.7 \\
\hline 6 & -- & -- & -- & -- & -- & -- & 14.8 & 13.9 & 14.3 & 18.2 & 17.2 & 17.7 \\
\hline 7 & -- & -- & -- & -- & -- & -- & 15.0 & 14.2 & 14.6 & 18.6 & 17.6 & 18.1 \\
\hline 8 & -- & -- & -- & -- & -- & -- & 15.3 & 14.5 & 14.9 & 19.0 & 18.1 & 18.6 \\
\hline 9 & -- & -- & -- & -- & -- & -- & 15.1 & 14.8 & 14.9 & 19.1 & 18.5 & 18.8 \\
\hline 10 & -- & -- & -- & -- & -- & -- & 15.1 & 14.5 & 14.8 & 19.1 & 18.4 & 18.7 \\
\hline 11 & -- & -- & -- & -- & -- & -- & 15.4 & 14.8 & 15.0 & 19.4 & 18.4 & 18.9 \\
\hline 12 & -- & -- & -- & -- & -- & -- & 15.7 & 14.8 & 15.2 & 20.0 & 18.8 & 19.4 \\
\hline 13 & -- & -- & -- & -- & -- & -- & 15.6 & 15.0 & 15.3 & 20.5 & 19.3 & 19.9 \\
\hline 14 & -- & -- & -- & -- & -- & -- & 15.7 & 15.0 & 15.4 & 21.0 & 19.9 & 20.4 \\
\hline 15 & -- & -- & -- & -- & -- & -- & 15.7 & 15.1 & 15.4 & 20.7 & 19.8 & 20.3 \\
\hline 16 & -- & -- & -- & -- & -- & -- & 15.5 & 15.0 & 15.2 & 20.4 & 19.8 & 20.1 \\
\hline 17 & -- & -- & -- & -- & -- & -- & 15.1 & 14.9 & 15.0 & 20.1 & 19.4 & 19.7 \\
\hline 18 & -- & -- & -- & -- & -- & -- & 15.2 & 14.7 & 15.0 & 19.6 & 18.7 & 19.0 \\
\hline 19 & -- & -- & -- & -- & -- & -- & 15.5 & 14.7 & 15.2 & 19.1 & 18.6 & 18.8 \\
\hline 20 & -- & -- & -- & -- & -- & -- & 15.9 & 15.0 & 15.5 & 19.4 & 18.6 & 19.0 \\
\hline 21 & -- & -- & -- & -- & -- & -- & 15.7 & 15.5 & 15.6 & 19.8 & 18.9 & 19.3 \\
\hline 22 & -- & -- & -- & -- & -- & -- & 15.5 & 15.2 & 15.4 & 20.4 & 19.2 & 19.8 \\
\hline 23 & -- & -- & -- & -- & -- & -- & 15.6 & 15.2 & 15.4 & 20.9 & 19.8 & 20.4 \\
\hline 24 & -- & -- & -- & -- & -- & -- & 15.7 & 15.3 & 15.5 & 21.2 & 20.3 & 20.8 \\
\hline 25 & -- & -- & -- & -- & -- & -- & 16.1 & 15.4 & 15.7 & 21.3 & 20.4 & 20.9 \\
\hline 26 & -- & -- & -- & -- & -- & -- & 16.7 & 15.7 & 16.2 & 21.6 & 20.6 & 21.1 \\
\hline 27 & -- & -- & -- & -- & -- & -- & 16.7 & 16.2 & 16.4 & 21.6 & 20.7 & 21.2 \\
\hline 28 & -- & -- & -- & -- & -- & -- & 16.3 & 15.9 & 16.1 & 21.5 & 21.0 & 21.1 \\
\hline 29 & -- & -- & -- & -- & -- & -- & 16.7 & 15.8 & 16.2 & 21.4 & 20.7 & 21.0 \\
\hline 30 & -- & -- & -- & 12.7 & 12.1 & 12.4 & 17.2 & 16.2 & 16.7 & 21.3 & 20.4 & 20.9 \\
\hline 31 & -- & -- & -- & 13.2 & 12.5 & 12.8 & -- & -- & -- & 21.1 & 20.5 & 20.8 \\
\hline Month & -- & -- & -- & -- & -- & -- & 17.2 & 12.8 & 15.1 & 21.6 & 16.5 & 19.4 \\
\hline \multirow{2}{*}{ Day } & \multicolumn{3}{|c|}{ August } & \multicolumn{3}{|c|}{ September } & & & & & & \\
\hline & Max & Min & Mean & Max & Min & Mean & & & & & & \\
\hline 1 & 20.8 & 20.4 & 20.6 & 20.7 & 20.0 & 20.4 & & & & & & \\
\hline 2 & 20.7 & 20.3 & 20.5 & 20.3 & 20.0 & 20.2 & & & & & & \\
\hline 3 & 20.6 & 20.1 & 20.4 & 20.2 & 19.9 & 20.1 & & & & & & \\
\hline 4 & 20.3 & 19.9 & 20.1 & 20.0 & 19.6 & 19.9 & & & & & & \\
\hline 5 & 20.3 & 19.8 & 20.1 & 19.7 & 19.3 & 19.6 & & & & & & \\
\hline 6 & 20.1 & 19.5 & 19.8 & 19.8 & 19.1 & 19.5 & & & & & & \\
\hline 7 & 20.6 & 19.6 & 20.1 & 20.0 & 19.2 & 19.5 & & & & & & \\
\hline 8 & 21.2 & 20.0 & 20.7 & 20.4 & 19.4 & 19.7 & & & & & & \\
\hline 9 & 21.5 & 20.5 & 21.1 & 20.0 & 19.4 & 19.7 & & & & & & \\
\hline 10 & 22.4 & 20.8 & 21.6 & 20.1 & 19.5 & 19.8 & & & & & & \\
\hline 11 & 22.0 & 21.1 & 21.6 & 20.2 & 19.7 & 19.9 & & & & & & \\
\hline 12 & 21.6 & 21.2 & 21.4 & 20.1 & 19.7 & 19.9 & & & & & & \\
\hline 13 & 21.7 & 20.7 & 21.2 & 20.0 & 19.4 & 19.8 & & & & & & \\
\hline 14 & 21.5 & 20.8 & 21.2 & 19.5 & 19.0 & 19.2 & & & & & & \\
\hline 15 & 21.5 & 20.6 & 21.0 & 19.0 & 18.7 & 18.9 & & & & & & \\
\hline 16 & 21.2 & 20.6 & 20.9 & & & & & & & & & \\
\hline 17 & 21.1 & 20.5 & 20.8 & & & & & & & & & \\
\hline 18 & 20.6 & 20.1 & 20.4 & & & & & & & & & \\
\hline 19 & 20.3 & 19.8 & 20.0 & & & & & & & & & \\
\hline 20 & 20.2 & 19.6 & 19.9 & & & & & & & & & \\
\hline 21 & 20.4 & 19.7 & 20.2 & & & & & & & & & \\
\hline 22 & 20.6 & 19.7 & 20.2 & & & & & & & & & \\
\hline 23 & 21.0 & 20.0 & 20.6 & & & & & & & & & \\
\hline 24 & 21.2 & 20.4 & 20.8 & & & & & & & & & \\
\hline 25 & 21.3 & 20.7 & 21.0 & & & & & & & & & \\
\hline 26 & 21.1 & 20.6 & 20.9 & & & & & & & & & \\
\hline 27 & 21.0 & 20.4 & 20.6 & & & & & & & & & \\
\hline 28 & 20.7 & 20.0 & 20.4 & & & & & & & & & \\
\hline 29 & 21.0 & 20.1 & 20.6 & & & & & & & & & \\
\hline 30 & 20.8 & 20.3 & 20.6 & & & & & & & & & \\
\hline 31 & 20.9 & 20.4 & 20.7 & & & & & & & & & \\
\hline Month & 22.4 & 19.5 & 20.6 & & & & & & & & & \\
\hline
\end{tabular}


Table 42. Daily summary of barometric pressure for the Columbia River, right bank, near Kalama, Washington, April - September 1996

[Values reported in millimeters of mercury; Max, maximum; Min, minimum; Month, monthly summary statistics; --, not available]

\begin{tabular}{|c|c|c|c|c|c|c|c|c|c|c|c|c|}
\hline \multirow{2}{*}{ Day } & \multicolumn{3}{|c|}{ April } & \multicolumn{3}{|c|}{ May } & \multicolumn{3}{|c|}{ June } & \multicolumn{3}{|c|}{ July } \\
\hline & Max & Min & Mean & Max & Min & Mean & Max & Min & Mean & Max & Min & Mean \\
\hline 1 & -- & -- & -- & -- & -- & -- & 768 & 765 & 766 & 764 & 759 & 762 \\
\hline 2 & -- & -- & -- & -- & -- & -- & 766 & 761 & 764 & 760 & 757 & 758 \\
\hline 3 & -- & -- & -- & -- & -- & -- & 767 & 763 & 765 & 763 & 757 & 759 \\
\hline 4 & -- & -- & -- & -- & -- & -- & 768 & 766 & 768 & 768 & 761 & 765 \\
\hline 5 & -- & -- & -- & -- & -- & -- & 768 & 763 & 766 & 769 & 766 & 767 \\
\hline 6 & -- & -- & -- & -- & -- & -- & 765 & 762 & 764 & 767 & 762 & 765 \\
\hline 7 & -- & -- & -- & -- & -- & -- & 766 & 764 & 764 & 763 & 758 & 761 \\
\hline 8 & -- & -- & -- & -- & -- & -- & 767 & 764 & 765 & 762 & 759 & 760 \\
\hline 9 & -- & -- & -- & -- & -- & -- & 769 & 766 & 767 & 767 & 762 & 765 \\
\hline 10 & -- & -- & -- & -- & -- & -- & 768 & 765 & 766 & 768 & 764 & 766 \\
\hline 11 & -- & -- & -- & -- & -- & -- & 769 & 765 & 767 & 766 & 761 & 765 \\
\hline 12 & -- & -- & -- & -- & -- & -- & 765 & 760 & 763 & 764 & 760 & 762 \\
\hline 13 & -- & -- & -- & -- & -- & -- & 768 & 762 & 765 & 761 & 756 & 759 \\
\hline 14 & -- & -- & -- & -- & -- & -- & 768 & 765 & 767 & 758 & 754 & 757 \\
\hline 15 & -- & -- & -- & -- & -- & -- & 766 & 763 & 765 & 761 & 756 & 758 \\
\hline 16 & -- & -- & -- & -- & -- & -- & 766 & 761 & 763 & 763 & 757 & 760 \\
\hline 17 & -- & -- & -- & -- & -- & -- & 767 & 764 & 765 & 761 & 757 & 759 \\
\hline 18 & -- & -- & -- & -- & -- & -- & 769 & 766 & 767 & 765 & 759 & 762 \\
\hline 19 & -- & -- & -- & -- & -- & -- & 767 & 758 & 762 & 768 & 764 & 766 \\
\hline 20 & -- & -- & -- & -- & -- & -- & 761 & 758 & 759 & 766 & 763 & 765 \\
\hline 21 & -- & -- & -- & -- & -- & -- & 763 & 761 & 762 & 765 & 763 & 765 \\
\hline 22 & -- & -- & -- & -- & -- & -- & 764 & 759 & 762 & 765 & 760 & 762 \\
\hline 23 & -- & -- & -- & -- & -- & -- & 760 & 756 & 757 & 762 & 757 & 760 \\
\hline 24 & -- & -- & -- & -- & -- & -- & 760 & 757 & 758 & 760 & 757 & 759 \\
\hline 25 & -- & -- & -- & -- & -- & -- & 761 & 757 & 759 & 759 & 754 & 757 \\
\hline 26 & -- & -- & -- & -- & -- & -- & 757 & 753 & 755 & 763 & 754 & 758 \\
\hline 27 & -- & -- & -- & -- & -- & -- & 763 & 755 & 759 & 763 & 758 & 761 \\
\hline 28 & -- & -- & -- & -- & -- & -- & 768 & 763 & 765 & 766 & 761 & 764 \\
\hline 29 & -- & -- & -- & -- & -- & -- & 767 & 764 & 766 & 766 & 762 & 764 \\
\hline 30 & -- & -- & -- & 765 & 763 & 764 & 765 & 762 & 764 & 764 & 761 & 763 \\
\hline 31 & -- & -- & -- & 766 & 763 & 764 & -- & -- & -- & 764 & 758 & 762 \\
\hline Month & -- & -- & -- & -- & -- & -- & 769 & 753 & 763 & 769 & 754 & 762 \\
\hline \multirow{2}{*}{ Day } & \multicolumn{3}{|c|}{ August } & \multicolumn{3}{|c|}{ September } & & & & & & \\
\hline & Max & Min & Mean & Max & Min & Mean & & & & & & \\
\hline 1 & 762 & 758 & 760 & 760 & 756 & 758 & & & & & & \\
\hline 2 & 762 & 756 & 760 & 758 & 755 & 757 & & & & & & \\
\hline 3 & 762 & 759 & 761 & 756 & 753 & 755 & & & & & & \\
\hline 4 & 760 & 755 & 758 & 756 & 751 & 754 & & & & & & \\
\hline 5 & 762 & 754 & 759 & 759 & 755 & 757 & & & & & & \\
\hline 6 & 763 & 758 & 761 & 759 & 755 & 757 & & & & & & \\
\hline 7 & 760 & 756 & 759 & 758 & 755 & 757 & & & & & & \\
\hline 8 & 762 & 758 & 760 & 759 & 756 & 757 & & & & & & \\
\hline 9 & 762 & 755 & 759 & 759 & 755 & 757 & & & & & & \\
\hline 10 & 756 & 750 & 753 & 757 & 752 & 755 & & & & & & \\
\hline 11 & 761 & 752 & 758 & 755 & 750 & 753 & & & & & & \\
\hline 12 & 762 & 758 & 760 & 754 & 750 & 752 & & & & & & \\
\hline 13 & 761 & 757 & 759 & 751 & 748 & 750 & & & & & & \\
\hline 14 & 765 & 758 & 761 & 750 & 742 & 748 & & & & & & \\
\hline 15 & 765 & 760 & 763 & 753 & 742 & 749 & & & & & & \\
\hline 16 & 763 & 760 & 762 & & & & & & & & & \\
\hline 17 & 763 & 760 & 762 & & & & & & & & & \\
\hline 18 & 764 & 760 & 762 & & & & & & & & & \\
\hline 19 & 760 & 756 & 759 & & & & & & & & & \\
\hline 20 & 763 & 758 & 761 & & & & & & & & & \\
\hline 21 & 764 & 759 & 762 & & & & & & & & & \\
\hline 22 & 761 & 756 & 759 & & & & & & & & & \\
\hline 23 & 758 & 755 & 757 & & & & & & & & & \\
\hline 24 & 757 & 752 & 754 & & & & & & & & & \\
\hline 25 & 755 & 751 & 752 & & & & & & & & & \\
\hline 26 & 754 & 750 & 752 & & & & & & & & & \\
\hline 27 & 758 & 752 & 755 & & & & & & & & & \\
\hline 28 & 760 & 756 & 757 & & & & & & & & & \\
\hline 29 & 759 & 754 & 757 & & & & & & & & & \\
\hline 30 & 759 & 754 & 757 & & & & & & & & & \\
\hline 31 & 761 & 758 & 759 & & & & & & & & & \\
\hline Month & 765 & 750 & 759 & & & & & & & & & \\
\hline
\end{tabular}


Table 43. Daily summary of total dissolved gas, in millimeters of mercury, for the Columbia River, right bank, near Kalama, Washington, April - September 1996

[Max, maximum; Min, minimum; Month, monthly summary statistics; --, not available; e, estimated]

\begin{tabular}{|c|c|c|c|c|c|c|c|c|c|c|c|c|}
\hline \multirow{2}{*}{ Day } & \multicolumn{3}{|c|}{ April } & \multicolumn{3}{|c|}{ May } & \multicolumn{3}{|c|}{ June } & \multicolumn{3}{|c|}{ July } \\
\hline & Max & Min & Mean & Max & Min & Mean & Max & Min & Mean & Max & Min & Mean \\
\hline 1 & -- & -- & -- & -- & -- & -- & 961 & 928 & 946 & 905 & 883 & 894 \\
\hline 2 & -- & -- & -- & -- & -- & -- & 981 & 927 & 958 & 904 & 887 & 896 \\
\hline 3 & -- & -- & -- & -- & -- & -- & 978 & 935 & 961 & 891 & 872 & 878 \\
\hline 4 & -- & -- & -- & -- & -- & -- & 954 & 930 & 941 & 873 & 857 & 863 \\
\hline 5 & -- & -- & -- & -- & -- & -- & 930 & 917 & 921 & 860 & 847 & 855 \\
\hline 6 & -- & -- & -- & -- & -- & -- & 942 & 919 & 930 & 865 & 850 & 858 \\
\hline 7 & -- & -- & -- & -- & -- & -- & 951 & 932 & 942 & 880 & 845 & 862 \\
\hline 8 & -- & -- & -- & -- & -- & -- & 937 & 917 & 924 & 878 & 862 & 869 \\
\hline 9 & -- & -- & -- & -- & -- & -- & 931 & 921 & 927 & 878 & 864 & 871 \\
\hline 10 & -- & -- & -- & -- & -- & -- & 946 & 928 & 940 & 871 & 852 & 858 \\
\hline 11 & -- & -- & -- & -- & -- & -- & 953 & 934 & 945 & 857 & 837 & 847 \\
\hline 12 & -- & -- & -- & -- & -- & -- & 941 & 926 & 933 & 875 & 850 & 863 \\
\hline 13 & -- & -- & -- & -- & -- & -- & 939 & 928 & 934 & 888 & 863 & 877 \\
\hline 14 & -- & -- & -- & -- & -- & -- & 933 & 908 & 925 & 904 & 879 & 891 \\
\hline 15 & -- & -- & -- & -- & -- & -- & 935 & 907 & 921 & 894 & 864 & 875 \\
\hline 16 & -- & -- & -- & -- & -- & -- & 923 & 898 & 907 & 885 & 869 & 876 \\
\hline 17 & -- & -- & -- & -- & -- & -- & 975 & 903 & 940 & 870 & 846 & 856 \\
\hline 18 & -- & -- & -- & -- & -- & -- & 953 & 914 & 935 & 846 & 829 & 834 \\
\hline 19 & -- & -- & -- & -- & -- & -- & 953 & 924 & 937 & 856 & 827 & 841 \\
\hline 20 & -- & -- & -- & -- & -- & -- & 931 & 913 & 922 & 879 & 851 & 865 \\
\hline 21 & -- & -- & -- & -- & -- & -- & 933 & 912 & 925 & 874 & 855 & 865 \\
\hline 22 & -- & -- & -- & -- & -- & -- & 919 & 893 & 910 & 870 & 843 & 856 \\
\hline 23 & -- & -- & -- & -- & -- & -- & 893 & 877 & 884 & 882 & 859 & 869 \\
\hline 24 & -- & -- & -- & -- & -- & -- & 901 & 872 & 885 & 887 & 862 & 874 \\
\hline 25 & -- & -- & -- & -- & -- & -- & 911 & 877 & 898 & 889 & 870 & 880 \\
\hline 26 & -- & -- & -- & -- & -- & -- & 943 & 899 & 920 & 887 & 866 & 877 \\
\hline 27 & -- & -- & -- & -- & -- & -- & 957 & 906 & 930 & 891 & 866 & 880 \\
\hline 28 & -- & -- & -- & -- & -- & -- & 926 & 896 & 910 & 886 & 868 & 878 \\
\hline 29 & -- & -- & -- & -- & -- & -- & 901 & 885 & 894 & 882 & 869 & 875 \\
\hline 30 & -- & -- & -- & 925 & 900 & 914 & 903 & 887 & 895 & 876 & 856 & 867 \\
\hline 31 & -- & -- & -- & 946 & 910 & 933 & -- & -- & -- & 865 & 849 & 856 \\
\hline Month & -- & -- & -- & -- & -- & -- & 981 & 872 & 925 & 905 & 827 & 868 \\
\hline \multirow{2}{*}{ Day } & \multicolumn{3}{|c|}{ August } & \multicolumn{3}{|c|}{ September } & & & & & & \\
\hline & Max & Min & Mean & Max & Min & Mean & & & & & & \\
\hline 1 & 856 & 840 & 848 & 841 & 834 & 838 & & & & & & \\
\hline 2 & 853 & 841 & 848 & 839 & 828 & 832 & & & & & & \\
\hline 3 & 848 & 830 & 839 & 829 & 807 & 816 & & & & & & \\
\hline 4 & 852 & 830 & 841 & 807 & 793 & 800 & & & & & & \\
\hline 5 & 851 & 839 & 844 & 801 & 791 & 796 & & & & & & \\
\hline 6 & 859 & 841 & 848 & 798 & 786 & 795 & & & & & & \\
\hline 7 & 860 & 835 & 848 & 802 & 793 & 797 & & & & & & \\
\hline 8 & 864 & 835 & 850 & 802 & 794 & 798 & & & & & & \\
\hline 9 & 871 & 847 & 860 & 798 & 791 & 795 & & & & & & \\
\hline 10 & 882 & 857 & 870 & 796 & 777 & 786 & & & & & & \\
\hline 11 & 878 & 853 & 867 & 781 & 776 & 779 & & & & & & \\
\hline 12 & 875 & 851 & 860 & 779 & 771 & 774 & & & & & & \\
\hline 13 & 864 & 835 & 849 & 774 & 769 & 771 & & & & & & \\
\hline 14 & 861 & 841 & 853 & 769 & 761 & 764 & & & & & & \\
\hline 15 & 858 & 843 & 850 & 762 & 755 & 759 & & & & & & \\
\hline 16 & 846 & 828 & 837 & & & & & & & & & \\
\hline 17 & 835 & 823 & 828 & & & & & & & & & \\
\hline 18 & 839 & 819 & 829 & & & & & & & & & \\
\hline 19 & 842 & 825 & 834 & & & & & & & & & \\
\hline 20 & 863 & 838 & 848 & & & & & & & & & \\
\hline 21 & 857 & 833 & 845 & & & & & & & & & \\
\hline 22 & 859 & 835 & 847 & & & & & & & & & \\
\hline 23 & 852 & 836 & 845 & & & & & & & & & \\
\hline 24 & 849 & 829 & 839 & & & & & & & & & \\
\hline 25 & 859 & 837 & 845 & & & & & & & & & \\
\hline 26 & 858 & 842 & 848 & & & & & & & & & \\
\hline 27 & 855 & 838 & 846 & & & & & & & & & \\
\hline 28 & 851 & 844 & 848 & & & & & & & & & \\
\hline 29 & 858 & 843 & 850 & & & & & & & & & \\
\hline 30 & 855 & 838 & 847 & & & & & & & & & \\
\hline 31 & 846 & 839 & 843 & & & & & & & & & \\
\hline Month & 882 & 819 & 847 & & & & & & & & & \\
\hline
\end{tabular}


Table 44. Daily summary of total dissolved gas, in percent saturation, for the Columbia River, right bank, near Kalama, Washington, April - September 1996

[Max, maximum; Min, minimum; Month, monthly summary statistics; --, not available]

\begin{tabular}{|c|c|c|c|c|c|c|c|c|c|c|c|c|}
\hline \multirow{2}{*}{ Day } & \multicolumn{3}{|c|}{ April } & \multicolumn{3}{|c|}{ May } & \multicolumn{3}{|c|}{ June } & \multicolumn{3}{|c|}{ July } \\
\hline & Max & Min & Mean & Max & Min & Mean & Max & Min & Mean & Max & Min & Mean \\
\hline 1 & -- & -- & -- & -- & -- & -- & 125 & 121 & 123 & 119 & 115 & 117 \\
\hline 2 & -- & -- & -- & -- & -- & -- & 128 & 121 & 125 & 119 & 117 & 118 \\
\hline 3 & -- & -- & -- & -- & -- & -- & 128 & 122 & 126 & 117 & 114 & 116 \\
\hline 4 & -- & -- & -- & -- & -- & -- & 124 & 121 & 123 & 115 & 112 & 113 \\
\hline 5 & -- & -- & -- & -- & -- & -- & 121 & 119 & 120 & 112 & 110 & 111 \\
\hline 6 & -- & -- & -- & -- & -- & -- & 123 & 120 & 122 & 113 & 111 & 112 \\
\hline 7 & -- & -- & -- & -- & -- & -- & 124 & 122 & 123 & 116 & 111 & 113 \\
\hline 8 & -- & -- & -- & -- & -- & -- & 123 & 120 & 121 & 116 & 113 & 114 \\
\hline 9 & -- & -- & -- & -- & -- & -- & 121 & 120 & 121 & 115 & 113 & 114 \\
\hline 10 & -- & -- & -- & -- & .- & -- & 124 & 121 & 123 & 114 & 111 & 112 \\
\hline 11 & -- & -- & -- & -- & -- & -- & 124 & 122 & 123 & 112 & 109 & 111 \\
\hline 12 & -- & -- & -- & -- & -- & -- & 123 & 121 & 122 & 115 & 111 & 113 \\
\hline 13 & -- & -- & -- & -- & -- & -- & 123 & 121 & 122 & 117 & 114 & 116 \\
\hline 14 & -- & -- & -- & -- & -- & -- & 121 & 119 & 121 & 120 & 116 & 118 \\
\hline 15 & -- & -- & -- & -- & -- & -- & 122 & 118 & 120 & 118 & 114 & 115 \\
\hline 16 & -- & -- & -- & -- & -- & -- & 121 & 118 & 119 & 117 & 114 & 115 \\
\hline 17 & -- & -- & -- & -- & -- & -- & 127 & 118 & 123 & 115 & 111 & 113 \\
\hline 18 & -- & -- & -- & -- & -- & -- & 124 & 119 & 122 & 111 & 108 & 109 \\
\hline 19 & -- & -- & -- & -- & -- & -- & 125 & 121 & 123 & 112 & 108 & 110 \\
\hline 20 & -- & -- & -- & -- & -- & -- & 122 & 120 & 121 & 115 & 111 & 113 \\
\hline 21 & -- & -- & -- & -- & -- & -- & 123 & 119 & 121 & 114 & 112 & 113 \\
\hline 22 & -- & -- & -- & -- & -- & -- & 120 & 117 & 119 & 114 & 110 & 112 \\
\hline 23 & -- & -- & -- & -- & -- & -- & 117 & 116 & 117 & 116 & 113 & 114 \\
\hline 24 & -- & -- & -- & -- & -- & -- & 119 & 115 & 117 & 117 & 113 & 115 \\
\hline 25 & -- & -- & -- & -- & -- & -- & 120 & 115 & 118 & 118 & 115 & 116 \\
\hline 26 & -- & -- & -- & -- & -- & -- & 125 & 119 & 122 & 117 & 114 & 116 \\
\hline 27 & -- & -- & -- & -- & -- & -- & 126 & 119 & 122 & 118 & 113 & 116 \\
\hline 28 & -- & -- & -- & -- & -- & -- & 121 & 117 & 119 & 116 & 113 & 115 \\
\hline 29 & -- & -- & -- & -- & -- & -- & 118 & 115 & 117 & 116 & 113 & 115 \\
\hline 30 & -- & -- & -- & 121 & 118 & 120 & 118 & 116 & 117 & 115 & 112 & 114 \\
\hline 31 & -- & -- & -- & 124 & 119 & 122 & -- & -- & -- & 113 & 111 & 112 \\
\hline Month & -- & -- & -- & -- & -- & -- & 128 & 115 & 121 & 120 & 108 & 114 \\
\hline \multirow{2}{*}{ Day } & \multicolumn{3}{|c|}{ August } & \multicolumn{3}{|c|}{ September } & & & & & & \\
\hline & Max & Min & Mean & Max & Min & Mean & & & & & & \\
\hline 1 & 113 & 110 & 112 & 111 & 110 & 110 & & & & & & \\
\hline 2 & 112 & 111 & 112 & 111 & 109 & 110 & & & & & & \\
\hline 3 & 112 & 109 & 110 & 110 & 107 & 108 & & & & & & \\
\hline 4 & 113 & 110 & 111 & 107 & 105 & 106 & & & & & & \\
\hline 5 & 113 & 110 & 111 & 106 & 104 & 105 & & & & & & \\
\hline 6 & 113 & 110 & 111 & 106 & 104 & 105 & & & & & & \\
\hline 7 & 114 & 110 & 112 & 106 & 105 & 105 & & & & & & \\
\hline 8 & 114 & 110 & 112 & 106 & 105 & 105 & & & & & & \\
\hline 9 & 115 & 111 & 113 & 105 & 104 & 105 & & & & & & \\
\hline 10 & 117 & 114 & 115 & 105 & 103 & 104 & & & & & & \\
\hline 11 & 117 & 112 & 114 & 104 & 103 & 103 & & & & & & \\
\hline 12 & 115 & 112 & 113 & 104 & 102 & 103 & & & & & & \\
\hline 13 & 114 & 110 & 112 & 103 & 102 & 103 & & & & & & \\
\hline 14 & 114 & 110 & 112 & 103 & 102 & 102 & & & & & & \\
\hline 15 & 113 & 110 & 111 & 102 & 101 & 101 & & & & & & \\
\hline 16 & 111 & 108 & 110 & & & & & & & & & \\
\hline 17 & 110 & 108 & 109 & & & & & & & & & \\
\hline 18 & 110 & 107 & 109 & & & & & & & & & \\
\hline 19 & 111 & 109 & 110 & & & & & & & & & \\
\hline 20 & 114 & 110 & 112 & & & & & & & & & \\
\hline 21 & 113 & 109 & 111 & & & & & & & & & \\
\hline 22 & 113 & 110 & 112 & & & & & & & & & \\
\hline 23 & 113 & 110 & 112 & & & & & & & & & \\
\hline 24 & 113 & 110 & 111 & & & & & & & & & \\
\hline 25 & 114 & 111 & 112 & & & & & & & & & \\
\hline 26 & 114 & 112 & 113 & & & & & & & & & \\
\hline 27 & 113 & 111 & 112 & & & & & & & & & \\
\hline 28 & 112 & 111 & 112 & & & & & & & & & \\
\hline 29 & 113 & 111 & 112 & & & & & & & & & \\
\hline 30 & 113 & 111 & 112 & & & & & & & & & \\
\hline 31 & 111 & 110 & 111 & & & & & & & & & \\
\hline Month & 117 & 107 & 112 & & & & & & & & & \\
\hline
\end{tabular}



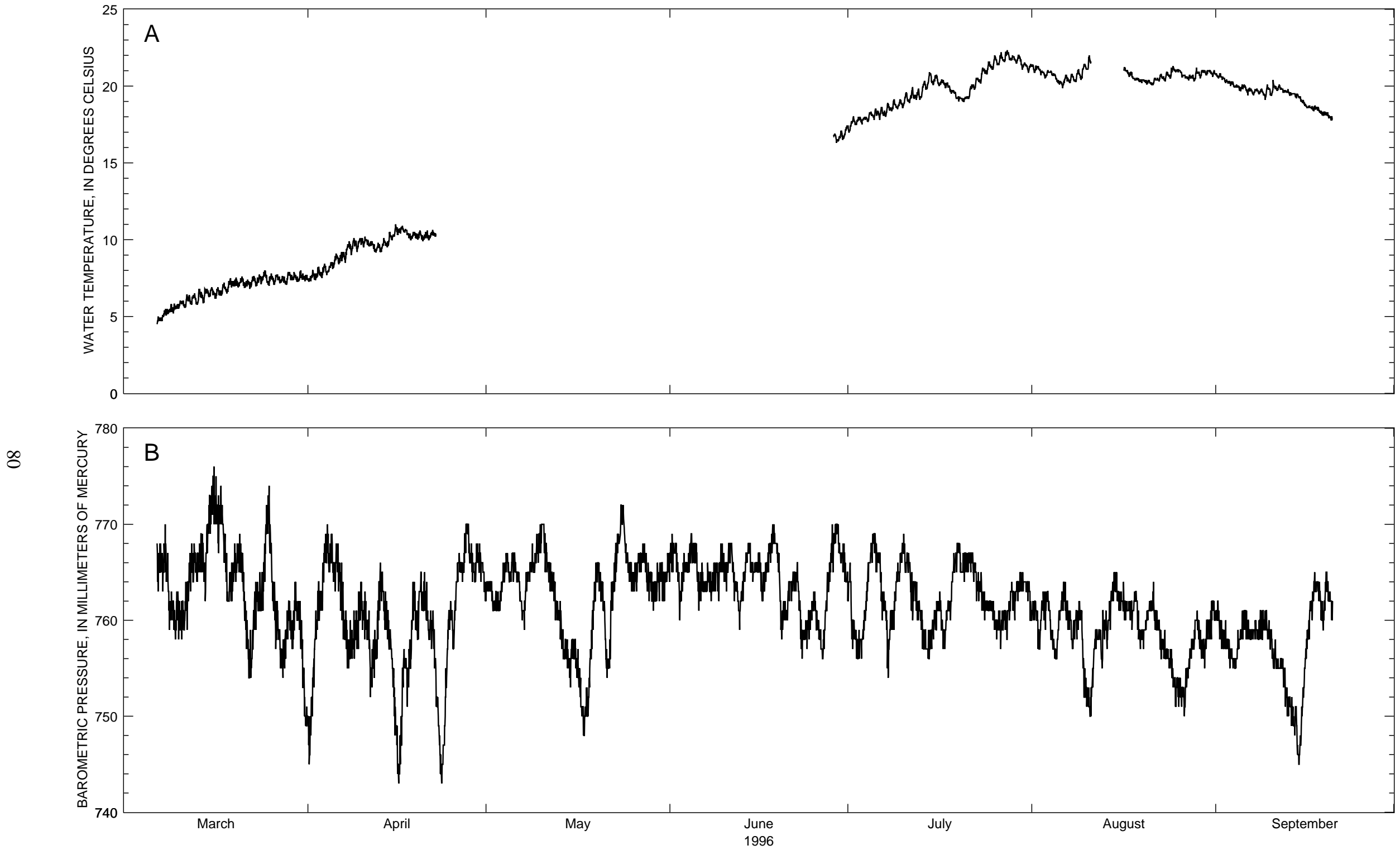

Figure 15. Hourly values of (A) water temperature, (B) barometric pressure, and (C, D) total dissolved gas at the Columbia River, left bank, at Wanna, Oregon, March September 1996. 

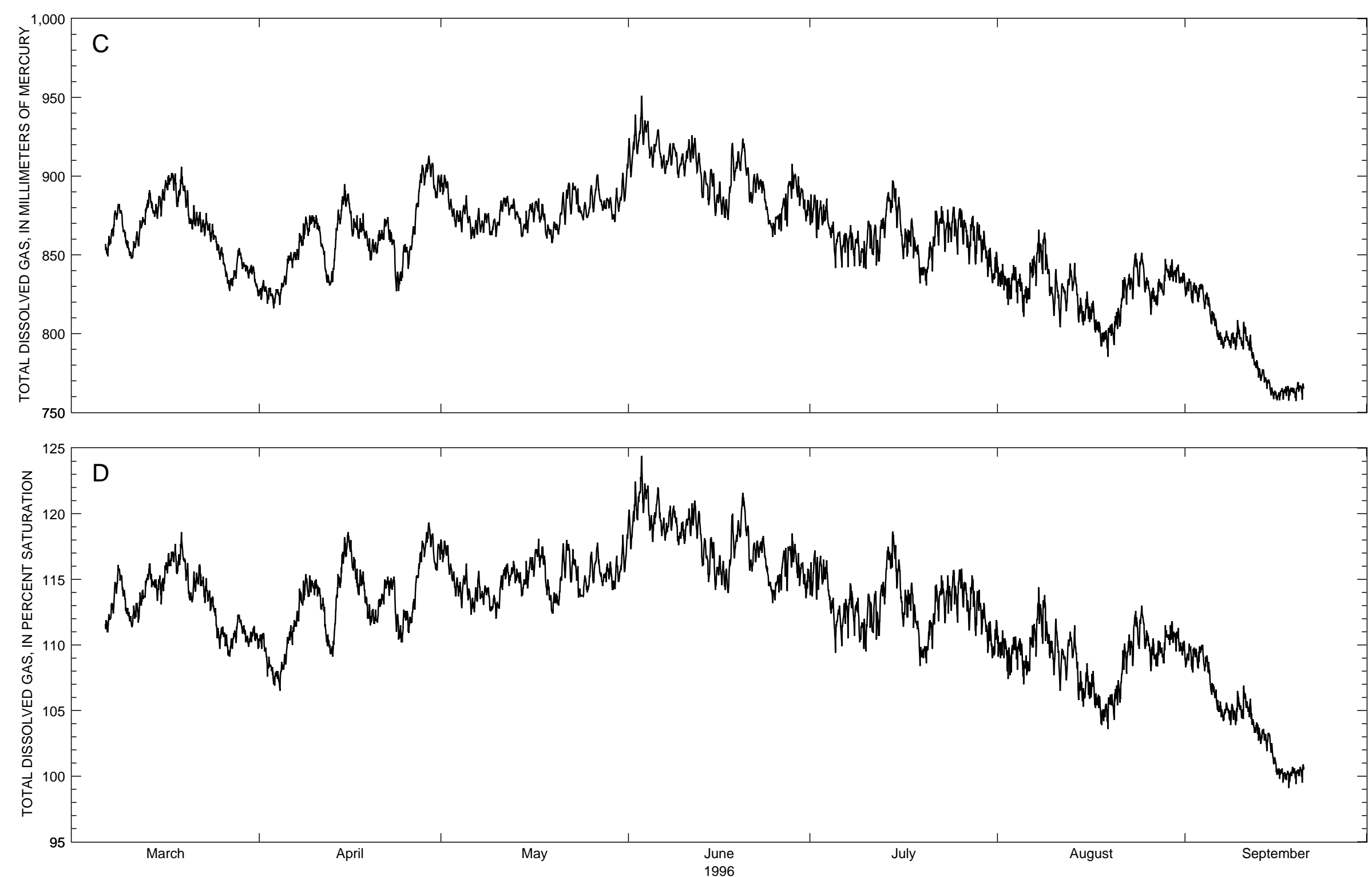

Figure 15. Hourly values of (A) water temperature, (B) barometric pressure, and (C, D) total dissolved gas at the Columbia River, left bank, at Wauna, Oregon, March September 1996.-continued 
Table 45. Daily summary of water temperature for the Columbia River, left bank, at Wauna, Oregon, March - September 1996

[Values reported in degrees Celsius; Max, maximum; Min, minimum; Month, monthly summary statistics; --, not available]

\begin{tabular}{|c|c|c|c|c|c|c|c|c|c|c|c|c|}
\hline \multirow{2}{*}{ Day } & \multicolumn{3}{|c|}{ March } & \multicolumn{3}{|c|}{ April } & \multicolumn{3}{|c|}{ May } & \multicolumn{3}{|c|}{ June } \\
\hline & Max & Min & Mean & Max & Min & Mean & Max & Min & Mean & Max & Min & Mean \\
\hline 1 & & & & 8.0 & 7.3 & 7.6 & -- & -- & -- & -- & -- & -- \\
\hline 2 & & & & 8.2 & 7.4 & 7.7 & -- & -- & -- & -- & -- & -- \\
\hline 3 & & & & 8.4 & 7.5 & 7.9 & -- & -- & -- & -- & -- & -- \\
\hline 4 & & & & 8.5 & 7.7 & 8.1 & -- & -- & -- & -- & -- & -- \\
\hline 5 & & & & 9.1 & 8.1 & 8.6 & -- & -- & -- & -- & -- & -- \\
\hline 6 & & & & 9.2 & 8.4 & 8.8 & -- & -- & -- & -- & -- & -- \\
\hline 7 & 5.4 & 4.7 & 5.0 & 9.9 & 8.5 & 9.2 & -- & -- & -- & -- & -- & -- \\
\hline 8 & 5.8 & 5.1 & 5.4 & 10.1 & 9.0 & 9.6 & -- & -- & -- & -- & -- & -- \\
\hline 9 & 5.8 & 5.2 & 5.5 & 10.1 & 9.2 & 9.8 & -- & -- & -- & -- & -- & -- \\
\hline 10 & 6.0 & 5.5 & 5.8 & 10.2 & 9.5 & 9.9 & -- & -- & -- & -- & -- & -- \\
\hline 11 & 6.4 & 5.6 & 6.0 & 10.0 & 9.6 & 9.7 & -- & -- & -- & -- & -- & -- \\
\hline 12 & 6.5 & 5.8 & 6.1 & 9.8 & 9.2 & 9.5 & -- & -- & -- & -- & -- & -- \\
\hline 13 & 6.8 & 5.8 & 6.2 & 10.1 & 9.2 & 9.6 & -- & -- & -- & -- & -- & -- \\
\hline 14 & 6.8 & 5.9 & 6.4 & 10.5 & 9.5 & 9.9 & -- & -- & -- & -- & -- & -- \\
\hline 15 & 6.9 & 6.3 & 6.6 & 11.0 & 10.0 & 10.4 & -- & -- & -- & -- & -- & -- \\
\hline 16 & 6.9 & 6.2 & 6.5 & 10.9 & 10.3 & 10.6 & -- & -- & -- & -- & -- & -- \\
\hline 17 & 7.2 & 6.4 & 6.7 & 10.7 & 10.2 & 10.5 & -- & -- & -- & -- & -- & -- \\
\hline 18 & 7.5 & 6.5 & 6.9 & 10.5 & 10.0 & 10.2 & -- & -- & -- & -- & -- & -- \\
\hline 19 & 7.5 & 6.9 & 7.2 & 10.6 & 10.0 & 10.3 & -- & -- & -- & -- & -- & -- \\
\hline 20 & 7.6 & 6.9 & 7.2 & 10.5 & 9.9 & 10.2 & -- & -- & -- & -- & -- & -- \\
\hline 21 & 7.5 & 6.9 & 7.2 & 10.6 & 10.0 & 10.3 & -- & -- & -- & -- & -- & -- \\
\hline 22 & 7.6 & 6.8 & 7.2 & -- & -- & -- & -- & -- & -- & -- & -- & -- \\
\hline 23 & 7.7 & 7.0 & 7.3 & -- & -- & -- & -- & -- & -- & -- & -- & -- \\
\hline 24 & 8.0 & 7.1 & 7.6 & -- & -- & -- & -- & -- & -- & -- & -- & -- \\
\hline 25 & 7.8 & 7.0 & 7.4 & -- & -- & -- & -- & -- & -- & -- & -- & -- \\
\hline 26 & 7.7 & 7.1 & 7.4 & -- & -- & -- & -- & -- & -- & -- & -- & -- \\
\hline 27 & 7.6 & 7.2 & 7.4 & -- & -- & -- & -- & -- & -- & -- & -- & -- \\
\hline 28 & 7.9 & 7.1 & 7.5 & -- & -- & -- & -- & -- & -- & -- & -- & -- \\
\hline 29 & 7.9 & 7.4 & 7.6 & -- & -- & -- & -- & -- & -- & 17.1 & 16.3 & 16.6 \\
\hline 30 & 8.0 & 7.3 & 7.5 & -- & -- & -- & -- & -- & -- & 17.4 & 16.5 & 17.0 \\
\hline 31 & 7.8 & 7.3 & 7.5 & -- & -- & -- & -- & -- & -- & -- & -- & -- \\
\hline Month & -- & -- & -- & -- & -- & -- & -- & -- & -- & -- & -- & -- \\
\hline \multirow{2}{*}{ Day } & \multicolumn{3}{|c|}{ July } & \multicolumn{3}{|c|}{ August } & \multicolumn{3}{|c|}{ September } & & & \\
\hline & Max & Min & Mean & Max & Min & Mean & Max & Min & Mean & & & \\
\hline 1 & 18.0 & 17.0 & 17.5 & 21.4 & 20.9 & 21.2 & 20.8 & 20.5 & 20.7 & & & \\
\hline 2 & 18.0 & 17.5 & 17.7 & 21.3 & 20.7 & 21.0 & 20.6 & 20.2 & 20.4 & & & \\
\hline 3 & 18.0 & 17.5 & 17.8 & 21.0 & 20.5 & 20.8 & 20.3 & 20.0 & 20.2 & & & \\
\hline 4 & 18.3 & 17.7 & 18.0 & 21.0 & 20.6 & 20.8 & 20.1 & 19.7 & 20.0 & & & \\
\hline 5 & 18.5 & 17.8 & 18.2 & 20.6 & 20.1 & 20.3 & 20.1 & 19.7 & 19.9 & & & \\
\hline 6 & 18.6 & 17.8 & 18.3 & 20.7 & 19.9 & 20.3 & 19.9 & 19.4 & 19.7 & & & \\
\hline 7 & 18.9 & 18.0 & 18.4 & 20.8 & 20.2 & 20.5 & 19.8 & 19.4 & 19.6 & & & \\
\hline 8 & 19.1 & 18.4 & 18.7 & 21.1 & 20.3 & 20.6 & 19.8 & 19.5 & 19.6 & & & \\
\hline 9 & 19.1 & 18.5 & 18.8 & 21.4 & 20.4 & 20.9 & 20.1 & 19.1 & 19.6 & & & \\
\hline 10 & 19.6 & 18.6 & 19.0 & 22.0 & 21.1 & 21.4 & 20.4 & 19.4 & 19.8 & & & \\
\hline 11 & 19.9 & 18.9 & 19.3 & -- & -- & -- & 20.1 & 19.7 & 19.9 & & & \\
\hline 12 & 19.8 & 19.1 & 19.4 & -- & -- & -- & 19.9 & 19.6 & 19.7 & & & \\
\hline 13 & 20.3 & 19.1 & 19.7 & -- & -- & -- & 19.8 & 19.4 & 19.5 & & & \\
\hline 14 & 20.9 & 19.8 & 20.3 & -- & -- & -- & 19.5 & 19.2 & 19.4 & & & \\
\hline 15 & 20.7 & 20.1 & 20.4 & -- & -- & -- & 19.3 & 18.7 & 19.1 & & & \\
\hline 16 & 20.6 & 20.0 & 20.2 & 21.2 & -- & -- & 18.8 & 18.6 & 18.7 & & & \\
\hline 17 & 20.4 & 19.9 & 20.1 & 21.0 & 20.6 & 20.8 & 18.7 & 18.4 & 18.6 & & & \\
\hline 18 & 20.0 & 19.5 & 19.7 & 20.6 & 20.4 & 20.5 & 18.6 & 18.1 & 18.4 & & & \\
\hline 19 & 19.6 & 19.0 & 19.3 & 20.5 & 20.2 & 20.4 & 18.3 & 18.0 & 18.2 & & & \\
\hline 20 & 19.3 & 19.0 & 19.2 & 20.4 & 20.1 & 20.3 & & & & & & \\
\hline 21 & 20.1 & 19.2 & 19.6 & 20.5 & 20.1 & 20.3 & & & & & & \\
\hline 22 & 20.5 & 19.8 & 20.2 & 20.8 & 20.3 & 20.5 & & & & & & \\
\hline 23 & 21.2 & 20.2 & 20.7 & 20.9 & 20.3 & 20.6 & & & & & & \\
\hline 24 & 21.6 & 20.8 & 21.1 & 21.3 & 20.5 & 20.9 & & & & & & \\
\hline 25 & 22.0 & 21.1 & 21.5 & 21.1 & 20.9 & 21.0 & & & & & & \\
\hline 26 & 22.2 & 21.4 & 21.7 & 21.0 & 20.5 & 20.7 & & & & & & \\
\hline 27 & 22.3 & 21.6 & 21.9 & 20.7 & 20.4 & 20.6 & & & & & & \\
\hline 28 & 22.2 & 21.7 & 21.9 & 21.2 & 20.3 & 20.7 & & & & & & \\
\hline 29 & 22.0 & 21.5 & 21.8 & 21.0 & 20.5 & 20.8 & & & & & & \\
\hline 30 & 21.8 & 21.1 & 21.4 & 21.0 & 20.7 & 20.9 & & & & & & \\
\hline 31 & 21.4 & 20.9 & 21.2 & 21.0 & 20.6 & 20.8 & & & & & & \\
\hline Month & 22.3 & 17.0 & 19.8 & -- & -- & -- & & & & & & \\
\hline
\end{tabular}


Table 46. Daily summary of barometric pressure for the Columbia River, left bank, at Wauna, Oregon, March - September 1996

[Values reported in millimeters of mercury; Max, maximum; Min, minimum; Month, monthly summary statistics; --, not available]

\begin{tabular}{|c|c|c|c|c|c|c|c|c|c|c|c|c|}
\hline \multirow{2}{*}{ Day } & \multicolumn{3}{|c|}{ March } & \multicolumn{3}{|c|}{ April } & \multicolumn{3}{|c|}{ May } & \multicolumn{3}{|c|}{ June } \\
\hline & Max & Min & Mean & Max & Min & Mean & Max & Min & Mean & Max & Min & Mean \\
\hline 1 & & & & 756 & 745 & 750 & 764 & 762 & 764 & 769 & 765 & 767 \\
\hline 2 & & & & 764 & 753 & 760 & 763 & 761 & 762 & 768 & 760 & 764 \\
\hline 3 & & & & 769 & 761 & 764 & 766 & 761 & 763 & 767 & 763 & 765 \\
\hline 4 & & & & 770 & 765 & 767 & 767 & 764 & 765 & 769 & 765 & 767 \\
\hline 5 & & & & 769 & 763 & 766 & 767 & 764 & 766 & 768 & 761 & 766 \\
\hline 6 & & & & 768 & 758 & 763 & 766 & 762 & 764 & 766 & 762 & 764 \\
\hline 7 & 770 & 763 & 766 & 761 & 755 & 758 & 765 & 759 & 762 & 766 & 762 & 764 \\
\hline 8 & 768 & 759 & 763 & 761 & 756 & 758 & 768 & 764 & 765 & 766 & 763 & 764 \\
\hline 9 & 763 & 758 & 761 & 764 & 757 & 760 & 769 & 765 & 767 & 768 & 762 & 765 \\
\hline 10 & 763 & 758 & 761 & 764 & 758 & 761 & 770 & 765 & 769 & 768 & 763 & 766 \\
\hline 11 & 766 & 759 & 762 & 761 & 752 & 757 & 768 & 763 & 766 & 768 & 762 & 766 \\
\hline 12 & 768 & 763 & 766 & 764 & 754 & 758 & 765 & 760 & 763 & 764 & 759 & 762 \\
\hline 13 & 769 & 764 & 766 & 766 & 759 & 763 & 762 & 757 & 760 & 767 & 762 & 765 \\
\hline 14 & 770 & 762 & 766 & 763 & 757 & 759 & 759 & 755 & 757 & 768 & 764 & 767 \\
\hline 15 & 775 & 767 & 771 & 757 & 747 & 753 & 758 & 753 & 756 & 767 & 764 & 766 \\
\hline 16 & 776 & 767 & 772 & 755 & 743 & 747 & 758 & 751 & 755 & 767 & 762 & 764 \\
\hline 17 & 774 & 766 & 770 & 757 & 751 & 755 & 753 & 748 & 750 & 768 & 764 & 766 \\
\hline 18 & 769 & 762 & 764 & 764 & 754 & 759 & 761 & 750 & 755 & 770 & 767 & 768 \\
\hline 19 & 768 & 762 & 765 & 764 & 758 & 761 & 766 & 759 & 764 & 768 & 758 & 764 \\
\hline 20 & 769 & 764 & 766 & 765 & 760 & 763 & 766 & 761 & 763 & 763 & 759 & 761 \\
\hline 21 & 767 & 756 & 760 & 764 & 757 & 759 & 761 & 754 & 756 & 765 & 762 & 764 \\
\hline 22 & 762 & 754 & 757 & 761 & 749 & 755 & 768 & 757 & 764 & 766 & 761 & 764 \\
\hline 23 & 765 & 759 & 762 & 749 & 743 & 746 & 772 & 766 & 769 & 761 & 756 & 758 \\
\hline 24 & 770 & 761 & 765 & 761 & 747 & 756 & 772 & 765 & 768 & 762 & 757 & 760 \\
\hline 25 & 774 & 764 & 769 & 764 & 757 & 760 & 766 & 763 & 765 & 763 & 759 & 761 \\
\hline 26 & 764 & 757 & 761 & 767 & 764 & 765 & 767 & 763 & 765 & 760 & 756 & 758 \\
\hline 27 & 759 & 754 & 757 & 770 & 764 & 768 & 768 & 765 & 767 & 766 & 757 & 762 \\
\hline 28 & 762 & 755 & 759 & 770 & 764 & 767 & 767 & 762 & 764 & 770 & 765 & 768 \\
\hline 29 & 764 & 757 & 760 & 768 & 764 & 766 & 766 & 761 & 763 & 770 & 766 & 768 \\
\hline 30 & 762 & 757 & 760 & 767 & 762 & 765 & 766 & 762 & 764 & 768 & 762 & 766 \\
\hline 31 & 758 & 749 & 752 & -- & -- & -- & 767 & 762 & 765 & -- & -- & -- \\
\hline Month & -- & -- & -- & 770 & 743 & 760 & 772 & 748 & 763 & 770 & 756 & 764 \\
\hline \multirow{2}{*}{ Day } & \multicolumn{3}{|c|}{ July } & \multicolumn{3}{|c|}{ August } & \multicolumn{3}{|c|}{ September } & & & \\
\hline & Max & Min & Mean & Max & Min & Mean & Max & Min & Mean & & & \\
\hline 1 & 766 & 757 & 762 & 762 & 760 & 761 & 762 & 757 & 760 & & & \\
\hline 2 & 760 & 756 & 758 & 763 & 757 & 760 & 760 & 757 & 758 & & & \\
\hline 3 & 761 & 756 & 759 & 763 & 759 & 762 & 758 & 755 & 757 & & & \\
\hline 4 & 768 & 761 & 765 & 761 & 756 & 758 & 759 & 755 & 756 & & & \\
\hline 5 & 769 & 765 & 768 & 762 & 756 & 759 & 761 & 758 & 759 & & & \\
\hline 6 & 767 & 760 & 764 & 764 & 760 & 762 & 761 & 758 & 759 & & & \\
\hline 7 & 763 & 754 & 759 & 762 & 757 & 759 & 760 & 758 & 759 & & & \\
\hline 8 & 763 & 758 & 760 & 762 & 758 & 760 & 761 & 758 & 759 & & & \\
\hline 9 & 767 & 762 & 765 & 762 & 752 & 758 & 761 & 758 & 759 & & & \\
\hline 10 & 769 & 765 & 767 & 754 & 750 & 752 & 759 & 755 & 757 & & & \\
\hline 11 & 767 & 760 & 764 & 759 & 752 & 756 & 758 & 755 & 755 & & & \\
\hline 12 & 764 & 760 & 762 & 761 & 755 & 758 & 756 & 751 & 754 & & & \\
\hline 13 & 762 & 757 & 760 & 761 & 757 & 759 & 753 & 749 & 751 & & & \\
\hline 14 & 759 & 756 & 757 & 765 & 759 & 762 & 751 & 745 & 749 & & & \\
\hline 15 & 762 & 757 & 760 & 765 & 761 & 763 & 755 & 745 & 750 & & & \\
\hline 16 & 763 & 759 & 761 & 764 & 760 & 762 & 762 & 755 & 758 & & & \\
\hline 17 & 762 & 757 & 760 & 762 & 759 & 761 & 765 & 760 & 763 & & & \\
\hline 18 & 767 & 760 & 763 & 763 & 758 & 762 & 764 & 760 & 763 & & & \\
\hline 19 & 768 & 765 & 767 & 759 & 757 & 758 & 765 & 759 & 762 & & & \\
\hline 20 & 767 & 764 & 766 & 762 & 759 & 760 & & & & & & \\
\hline 21 & 767 & 765 & 766 & 764 & 759 & 761 & & & & & & \\
\hline 22 & 767 & 762 & 765 & 761 & 756 & 759 & & & & & & \\
\hline 23 & 765 & 761 & 763 & 759 & 756 & 757 & & & & & & \\
\hline 24 & 763 & 760 & 762 & 757 & 753 & 755 & & & & & & \\
\hline 25 & 762 & 759 & 761 & 754 & 751 & 753 & & & & & & \\
\hline 26 & 761 & 757 & 759 & 755 & 750 & 752 & & & & & & \\
\hline 27 & 761 & 758 & 760 & 758 & 753 & 756 & & & & & & \\
\hline 28 & 764 & 760 & 762 & 761 & 757 & 759 & & & & & & \\
\hline 29 & 765 & 760 & 763 & 760 & 756 & 758 & & & & & & \\
\hline 30 & 765 & 763 & 764 & 760 & 755 & 758 & & & & & & \\
\hline 31 & 764 & 760 & 763 & 762 & 758 & 761 & & & & & & \\
\hline Month & 769 & 754 & 762 & 765 & 750 & 759 & & & & & & \\
\hline
\end{tabular}


Table 47. Daily summary of total dissolved gas, in millimeters of mercury, for the Columbia River, left bank, at Wauna, Oregon, March - September 1996

[Max, maximum; Min, minimum; Month, monthly summary statistics; --, not available; e, estimated]

\begin{tabular}{|c|c|c|c|c|c|c|c|c|c|c|c|c|}
\hline \multirow{2}{*}{ Day } & \multicolumn{3}{|c|}{ March } & \multicolumn{3}{|c|}{ April } & \multicolumn{3}{|c|}{ May } & \multicolumn{3}{|c|}{ June } \\
\hline & Max & Min & Mean & Max & Min & Mean & Max & Min & Mean & Max & Min & Mean \\
\hline 1 & & & & 834 & 822 & 828 & 901 & 888 & 895 & 926 & 899 & 913 \\
\hline 2 & & & & 830 & 819 & 826 & 897 & 874 & 884 & 939 & 914 & 924 \\
\hline 3 & & & & 828 & 816 & 823 & 879 & 868 & 874 & 951 & 920 & 933 \\
\hline 4 & & & & 832 & 818 & 827 & 882 & 864 & 875 & 935 & 906 & 921 \\
\hline 5 & & & & 849 & 830 & 839 & 888 & 860 & 875 & 930 & 906 & 920 \\
\hline 6 & & & & 852 & 842 & 848 & 872 & 860 & 865 & 927 & 904 & 912 \\
\hline 7 & 869 & 851 & 862 & 864 & 847 & 855 & 879 & 863 & 870 & 920 & 902 & 910 \\
\hline 8 & 882 & 868 & 877 & 874 & 856 & 865 & 876 & 864 & 873 & 921 & 907 & 915 \\
\hline 9 & 878 & 857 & 869 & 875 & 862 & 870 & 873 & 863 & 867 & 914 & 899 & 907 \\
\hline 10 & 859 & 848 & 853 & 875 & 864 & 869 & 884 & 861 & 871 & 923 & 900 & 910 \\
\hline 11 & 865 & 849 & 857 & 864 & 839 & 853 & 887 & 877 & 883 & 926 & 909 & 917 \\
\hline 12 & 874 & 856 & 868 & 840 & 830 & 834 & 887 & 875 & 881 & 923 & 902 & 910 \\
\hline 13 & 891 & 869 & 881 & 876 & 833 & 852 & 885 & 870 & 877 & 903 & 886 & 895 \\
\hline 14 & 887 & 874 & 879 & 887 & 870 & 878 & 873 & 862 & 867 & 904 & 882 & 894 \\
\hline 15 & 890 & 873 & 881 & 895 & 877 & 885 & 882 & 864 & 874 & 901 & 875 & 886 \\
\hline 16 & 900 & 884 & 892 & 878 & 862 & 869 & 884 & 871 & 879 & 896 & 874 & 883 \\
\hline 17 & 902 & 891 & 898 & 875 & 860 & 867 & 886 & 871 & 877 & 905 & 872 & 884 \\
\hline 18 & 902 & 881 & 889 & 876 & 855 & 864 & 876 & 860 & 867 & 921 & 897 & 908 \\
\hline 19 & 906 & 880 & 893 & 860 & 846 & 854 & 871 & 857 & 865 & 924 & 904 & 913 \\
\hline 20 & 891 & 866 & 875 & 866 & 851 & 857 & 879 & 863 & 870 & 921 & 886 & 907 \\
\hline 21 & 881 & 868 & 873 & 873 & 860 & 865 & 891 & 869 & 883 & 901 & 883 & 890 \\
\hline 22 & 877 & 862 & 870 & 874 & 856 & 865 & 896 & 876 & 888 & 902 & 889 & 896 \\
\hline 23 & 876 & 859 & 867 & 859 & 827 & 844 & 893 & 877 & 886 & 896 & 877 & 887 \\
\hline 24 & 870 & 854 & 862 & 856 & 827 & 841 & -- & -- & -- & 877 & 861 & 869 \\
\hline 25 & 857 & 847 & 852 & 857 & 841 & 851 & 894 & 873 & 881 & 876 & 863 & 871 \\
\hline 26 & 849 & 829 & 839 & 882 & 853 & 865 & 901 & 877 & 889 & e889 & 865 & 878 \\
\hline 27 & 840 & 827 & 834 & 907 & 880 & 893 & 896 & 878 & 885 & 896 & 868 & 888 \\
\hline 28 & 854 & 835 & 847 & 913 & 894 & 903 & 886 & 879 & 883 & 908 & 887 & 897 \\
\hline 29 & 848 & 839 & 843 & 913 & 892 & 903 & 889 & 871 & 878 & 900 & 880 & 889 \\
\hline 30 & 844 & 835 & 840 & 901 & 886 & 893 & 898 & 877 & 885 & 885 & 871 & 879 \\
\hline 31 & 843 & 824 & 831 & -- & -- & -- & 915 & 884 & 897 & -- & -- & -- \\
\hline Month & -- & -- & -- & 913 & 816 & 860 & -- & -- & -- & 951 & 861 & 900 \\
\hline \multirow{2}{*}{ Day } & \multicolumn{3}{|c|}{ July } & \multicolumn{3}{|c|}{ August } & \multicolumn{3}{|c|}{ September } & & & \\
\hline & Max & Min & Mean & Max & Min & Mean & Max & Min & Mean & & & \\
\hline 1 & 888 & 869 & 879 & 844 & 830 & 836 & 835 & 824 & 830 & & & \\
\hline 2 & 885 & 861 & 875 & 837 & 818 & 831 & 832 & 819 & 827 & & & \\
\hline 3 & e886 & 869 & 876 & 843 & 822 & 834 & 831 & 820 & 827 & & & \\
\hline 4 & -- & -- & -- & 842 & 819 & 832 & 826 & 816 & 822 & & & \\
\hline 5 & -- & -- & -- & 832 & 811 & 823 & 819 & 805 & 811 & & & \\
\hline 6 & 864 & 842 & 856 & 849 & 822 & 836 & 810 & 793 & 800 & & & \\
\hline 7 & 869 & 842 & 859 & 866 & 830 & 849 & 802 & 791 & 796 & & & \\
\hline 8 & 865 & 843 & 856 & 864 & 838 & 854 & 801 & 791 & 796 & & & \\
\hline 9 & 859 & 842 & 850 & 850 & 824 & 836 & 809 & 790 & 798 & & & \\
\hline 10 & 872 & 841 & 859 & 841 & 811 & 826 & 807 & 790 & 798 & & & \\
\hline 11 & 869 & 843 & 860 & 832 & 804 & 822 & 803 & 789 & 795 & & & \\
\hline 12 & 872 & 843 & 859 & 844 & 814 & 827 & 791 & 777 & 783 & & & \\
\hline 13 & 889 & 861 & 875 & 845 & 827 & 835 & 779 & 770 & 775 & & & \\
\hline 14 & 897 & 877 & 887 & 831 & 807 & 818 & 775 & 765 & 770 & & & \\
\hline 15 & 892 & 867 & 879 & 827 & 805 & 815 & 768 & 759 & 763 & & & \\
\hline 16 & 868 & 847 & 859 & 821 & 807 & 814 & 764 & 758 & 761 & & & \\
\hline 17 & 873 & 849 & 863 & 809 & 799 & 805 & 766 & 758 & 764 & & & \\
\hline 18 & 866 & 848 & 855 & 802 & 792 & 798 & 766 & 757 & 764 & & & \\
\hline 19 & e849 & 832 & 840 & 806 & 785 & 800 & 769 & 757 & 764 & & & \\
\hline 20 & e858 & 831 & 844 & 816 & 793 & 805 & & & & & & \\
\hline 21 & 878 & 839 & 861 & 836 & 805 & 819 & & & & & & \\
\hline 22 & 881 & 856 & 870 & 837 & 818 & 830 & & & & & & \\
\hline 23 & 879 & 852 & 866 & 851 & 822 & 839 & & & & & & \\
\hline 24 & 880 & 858 & 870 & 851 & 830 & 842 & & & & & & \\
\hline 25 & 879 & 850 & 869 & 845 & 826 & 834 & & & & & & \\
\hline 26 & 878 & 855 & 867 & 829 & 812 & 823 & & & & & & \\
\hline 27 & 875 & 844 & 861 & 835 & 818 & 826 & & & & & & \\
\hline 28 & 871 & 844 & 860 & e847 & 824 & 833 & & & & & & \\
\hline 29 & 865 & 846 & 857 & 847 & 834 & 840 & & & & & & \\
\hline 30 & 855 & 836 & 847 & 844 & 832 & 839 & & & & & & \\
\hline 31 & 852 & 832 & 841 & 838 & 828 & 835 & & & & & & \\
\hline Month & -- & -- & -- & 866 & 785 & 828 & & & & & & \\
\hline
\end{tabular}


Table 48. Daily summary of total dissolved gas, in percent saturation, for the Columbia River, left bank, at Wauna, Oregon, March - September 1996

[Max, maximum; Min, minimum; Month, monthly summary statistics; --, not available; e, estimated]

\begin{tabular}{|c|c|c|c|c|c|c|c|c|c|c|c|c|}
\hline \multirow{2}{*}{ Day } & \multicolumn{3}{|c|}{ March } & \multicolumn{3}{|c|}{ April } & \multicolumn{3}{|c|}{ May } & \multicolumn{3}{|c|}{ June } \\
\hline & Max & Min & Mean & Max & Min & Mean & Max & Min & Mean & Max & Min & Mean \\
\hline 1 & & & & 111 & 109 & 110 & 118 & 116 & 117 & 121 & 117 & 119 \\
\hline 2 & & & & 110 & 108 & 109 & 118 & 115 & 116 & 122 & 119 & 121 \\
\hline 3 & & & & 108 & 107 & 108 & 116 & 114 & 115 & 124 & 120 & 122 \\
\hline 4 & & & & 109 & 106 & 108 & 115 & 113 & 114 & 122 & 118 & 120 \\
\hline 5 & & & & 111 & 108 & 110 & 116 & 112 & 114 & 122 & 118 & 120 \\
\hline 6 & & & & 112 & 110 & 111 & 114 & 112 & 113 & 121 & 118 & 119 \\
\hline 7 & 113 & 111 & 112 & 114 & 111 & 113 & 116 & 113 & 114 & 121 & 118 & 119 \\
\hline 8 & 116 & 113 & 115 & 115 & 113 & 114 & 114 & 113 & 114 & 121 & 119 & 120 \\
\hline 9 & 115 & 113 & 114 & 115 & 114 & 114 & 114 & 113 & 113 & 119 & 118 & 118 \\
\hline 10 & 113 & 111 & 112 & 115 & 114 & 114 & 115 & 112 & 113 & 120 & 118 & 119 \\
\hline 11 & 113 & 111 & 112 & 114 & 111 & 113 & 116 & 114 & 115 & 121 & 118 & 120 \\
\hline 12 & 114 & 112 & 113 & 111 & 109 & 110 & 116 & 114 & 115 & 121 & 118 & 119 \\
\hline 13 & 116 & 114 & 115 & 115 & 109 & 112 & 116 & 115 & 115 & 118 & 116 & 117 \\
\hline 14 & 115 & 114 & 115 & 117 & 114 & 116 & 115 & 114 & 115 & 118 & 115 & 117 \\
\hline 15 & 115 & 113 & 114 & 119 & 117 & 118 & 117 & 114 & 116 & 118 & 114 & 116 \\
\hline 16 & 117 & 114 & 116 & 118 & 115 & 116 & 117 & 115 & 116 & 117 & 114 & 116 \\
\hline 17 & 117 & 116 & 117 & 116 & 114 & 115 & 118 & 116 & 117 & 118 & 114 & 115 \\
\hline 18 & 118 & 115 & 116 & 116 & 112 & 114 & 117 & 113 & 115 & 120 & 117 & 118 \\
\hline 19 & 119 & 115 & 117 & 113 & 111 & 112 & 114 & 112 & 113 & 122 & 118 & 120 \\
\hline 20 & 116 & 113 & 114 & 113 & 112 & 112 & 115 & 113 & 114 & 121 & 116 & 119 \\
\hline 21 & 116 & 113 & 115 & 115 & 113 & 114 & 118 & 115 & 117 & 118 & 116 & 117 \\
\hline 22 & 116 & 113 & 115 & 115 & 114 & 115 & 118 & 115 & 116 & 118 & 116 & 117 \\
\hline 23 & 115 & 113 & 114 & 115 & 111 & 113 & 116 & 114 & 115 & 118 & 115 & 117 \\
\hline 24 & 114 & 111 & 113 & 113 & 110 & 111 & -- & -- & -- & 115 & 113 & 114 \\
\hline 25 & 111 & 110 & 111 & 113 & 111 & 112 & 117 & 114 & 115 & 115 & 113 & 115 \\
\hline 26 & 111 & 109 & 110 & 115 & 112 & 113 & 118 & 115 & 116 & e117 & 114 & 116 \\
\hline 27 & 111 & 109 & 110 & 118 & 115 & 116 & 117 & 114 & 115 & 117 & 114 & 117 \\
\hline 28 & 112 & 110 & 112 & 119 & 117 & 118 & 116 & 115 & 116 & 118 & 115 & 117 \\
\hline 29 & 112 & 110 & 111 & 119 & 116 & 118 & 116 & 114 & 115 & 117 & 114 & 116 \\
\hline 30 & 111 & 110 & 111 & 118 & 116 & 117 & 117 & 115 & 116 & 116 & 114 & 115 \\
\hline 31 & 111 & 110 & 111 & -- & -- & -- & 119 & 116 & 117 & -- & -- & -- \\
\hline Month & -- & -- & -- & 119 & 106 & 113 & -- & -- & -- & 124 & 113 & 118 \\
\hline \multirow{2}{*}{ Day } & \multicolumn{3}{|c|}{ July } & \multicolumn{3}{|c|}{ August } & \multicolumn{3}{|c|}{ September } & & & \\
\hline & Max & Min & Mean & Max & Min & Mean & Max & Min & Mean & & & \\
\hline 1 & 117 & 114 & 115 & 111 & 109 & 110 & 110 & 108 & 109 & & & \\
\hline 2 & 117 & 113 & 115 & 110 & 107 & 109 & 110 & 108 & 109 & & & \\
\hline 3 & e116 & 114 & 115 & 111 & 108 & 109 & 110 & 108 & 109 & & & \\
\hline 4 & -- & -- & -- & 111 & 108 & 110 & 109 & 108 & 109 & & & \\
\hline 5 & -- & -- & -- & 110 & 107 & 108 & 108 & 106 & 107 & & & \\
\hline 6 & 113 & 110 & 112 & 111 & 108 & 110 & 107 & 104 & 105 & & & \\
\hline 7 & 115 & 110 & 113 & 114 & 109 & 112 & 106 & 104 & 105 & & & \\
\hline 8 & 114 & 111 & 113 & 114 & 110 & 112 & 105 & 104 & 105 & & & \\
\hline 9 & 112 & 110 & 111 & 112 & 109 & 110 & 106 & 104 & 105 & & & \\
\hline 10 & 114 & 109 & 112 & 112 & 108 & 110 & 107 & 104 & 105 & & & \\
\hline 11 & 114 & 110 & 113 & 110 & 106 & 109 & 106 & 104 & 105 & & & \\
\hline 12 & 115 & 110 & 113 & 111 & 107 & 109 & 105 & 103 & 104 & & & \\
\hline 13 & 117 & 113 & 115 & 111 & 109 & 110 & 104 & 102 & 103 & & & \\
\hline 14 & 119 & 116 & 117 & 109 & 106 & 107 & 103 & 102 & 103 & & & \\
\hline 15 & 118 & 114 & 116 & 109 & 105 & 107 & 103 & 101 & 102 & & & \\
\hline 16 & 114 & 111 & 113 & 108 & 106 & 107 & 101 & 100 & 100 & & & \\
\hline 17 & 115 & 112 & 114 & 106 & 105 & 106 & 101 & 100 & 100 & & & \\
\hline 18 & 114 & 111 & 112 & 105 & 104 & 105 & 101 & 99 & 100 & & & \\
\hline 19 & e111 & 108 & 109 & 106 & 104 & 105 & 101 & 99 & 100 & & & \\
\hline 20 & e112 & 109 & 110 & 107 & 104 & 106 & & & & & & \\
\hline 21 & 115 & 110 & 112 & 110 & 106 & 108 & & & & & & \\
\hline 22 & 115 & 112 & 114 & 111 & 108 & 109 & & & & & & \\
\hline 23 & 115 & 112 & 114 & 113 & 108 & 111 & & & & & & \\
\hline 24 & 116 & 113 & 114 & 113 & 110 & 111 & & & & & & \\
\hline 25 & 116 & 112 & 114 & 112 & 110 & 111 & & & & & & \\
\hline 26 & 116 & 113 & 114 & 110 & 108 & 109 & & & & & & \\
\hline 27 & 115 & 111 & 113 & 110 & 108 & 109 & & & & & & \\
\hline 28 & 114 & 111 & 113 & e111 & 109 & 110 & & & & & & \\
\hline 29 & 113 & 111 & 112 & 112 & 110 & 111 & & & & & & \\
\hline 30 & 112 & 109 & 111 & 111 & 109 & 111 & & & & & & \\
\hline 31 & 112 & 109 & 110 & 110 & 109 & 110 & & & & & & \\
\hline Month & -- & -- & -- & 114 & 104 & 109 & & & & & & \\
\hline
\end{tabular}

